\title{
Gli uccelli del Parco Regionale della Maremma e aree limitrofe (Grosseto, Toscana, Italia)
}

\author{
Pietro Giovacchini
}

Riassunto - Il Parco Regionale della Maremma è una area protetta di 8902 ha nel settore costiero centrale della provincia di Grosseto, Toscana meridionale. Gli habitat che rappresentano l'area protetta possono essere così brevemente descritti: foce del fiume Ombrone e zone umide della Palude della Trappola; Monti dell'Uccellina (cima più elevata: Poggio Lecci, 417 m s.l.m.); un ampio comprensorio interno e pianeggiante dove coesistono attività zootecniche e coltivazioni agricole. Lo sviluppo costiero raggiunge i $25 \mathrm{~km}$ circa. Sono presenti 6 Siti della Rete Natura 2000 classificati come ZSC - Zona Speciale di Conservazione - secondo la Direttiva "Habitat" 92/43/ CE e/o come ZPS - Zona di Protezione Speciale - secondo la Direttiva "Uccelli" 2009/147/CE. La maggior parte dell'area presenta una scarsa rete viaria e limitati insediamenti umani con copertura boschiva in larga parte dovuta a formazioni vegetali sclerofilliche mediterranee. In questo lavoro viene presentato un elenco commentato degli uccelli noti per l'area protetta a partire dalla sua istituzione avvenuta il 05/06/1975 sino al 31/12/2018. In totale, le specie di Uccelli rilevate sono $295(62,1 \%$ delle specie della Toscana ed il $52,5 \%$ delle specie note in Italia), mentre le specie nidificanti certe o probabili in tempi recenti (2008-2018) sono 84. Le specie di interesse comunitario presenti nell'Allegato I della Direttiva "Uccelli" 2009/147/CE sono 88, mentre quelle classificate come SPEC 1-3 "Species of European Conservation Concern" sono 33. L'area, con le zone umide "Bocca d'Ombrone" e "La Trappola" comprese nella Macrozona "Maremma Grossetana", ricade all'interno della qualifica di sito di importanza internazionale per lo svernamento di Anser anser e Mareca penelope; inoltre è sito di importanza nazionale per Mareca strepera, Anas crecca, Anas acuta, Spatula clypeata, Ardea alba, Platalea leucorodia, Phoenicopterus roseus, Recurvirostra avosetta, Pluvialis apricaria, Calidris alpina, Vanellus vanellus, Gallinago gallinago, Numenius arquata, Tringa erythropus e Tringa totanus. L'area protetta è di importanza per specie nidificanti in Direttiva 2009/147/CE legate agli specchi lacustri costieri e agli spazi marini, ad incolti, pascoli, così come a zone con vegetazione arborea e arbustiva sparsa su affioramenti rocciosi, margini di boschi, ambienti parzialmente allagati ed ai litorali sabbiosi, quali ad esempio, con dati minimi, Pandion haliaetus (1 coppia), Burhinus oedicnemus (7-8 coppie), Charadrius alexandrinus (1 coppia), Caprimulgus europaeus (25 coppie), Coracias garrulus (13-15 coppie), Lanius collurio (1-5 coppie), Lullula arborea

Regione Toscana, Direzione Ambiente e Energia, Settore Tutela della Natura e del Mare, strada Cavour 16, 58100 Grosseto, Italia.

E-mail: pietro.giovacchini@regione.toscana.it

(C) 2019 Pietro Giovacchini

Received: 6 September 2018

Accepted for publication: 9 May 2019

Online publication: 4 November 2019
(10 coppie) e Anthus campestris (6-7 coppie). Dal 2015 si segnala il consolidamento della nidificazione di Ardea cinerea, rilevandone per la prima volta la riproduzione come garzaia.

Parole chiave: conservazione, elenco delle specie, Parco Regionale della Maremma, rilevanza ornitologica, uccelli.

\begin{abstract}
The Birds of the Maremma Regional Park and adjacent areas (Grosseto, Tuscany, Italy).

The Parco Regionale della Maremma (Maremma Regional Park) is a protected area of 8902 ha in the central coastal sector of the province of Grosseto, southern Tuscany. The existing habitats include the mouth and final stretch of River Ombrone, as well as the Palude della Trappola wetland; the Monti dell'Uccellina hills with the highest elevation point recorded in the area (417 $\mathrm{m}$ a.s.1.) and an extensive area where agriculture and free-range livestock coexist. Inside the protected area, 6 sites are included in the Nature 2000 Network; among these 4 are classified as ZSC (Special Conservation Zone, according to EU Habitat Directive 92/43/CE) and 5 as ZPS (Special Protection Zone, according to EU Birds Directive 2009/147/CE). A scanty road network linking isolated human settlements intersects the area. The dominant vegetation is represented by a scrubby forest of Mediterranean sclerophylls. In this paper, we present a checklist of the bird fauna of the Maremma Regional Park, as recorded from the date of establishment of the protected area (Tuscany Regional Law n. 65 of 5 June 1975) until 31 December 2018. The observed species are 295 , i.e. $62.1 \%$ of the species known for Tuscany and $52.5 \%$ of those known for Italy, as a whole. Of these species, 84 were recently assigned the Confirmed or Probable Breeding status (2008-2018), 88 are considered of Community Interest (Annex 1 EU Birds Directive 2009/147/CE), and 33 breeding species are classified as SPEC 1-3 (Species of European Conservation Concern). With the wetland sites "Bocca d'Ombrone" and "La Trappola" included in the Macrozona "Maremma Grossetana", the Maremma Regional Park is considered as a wetland of international importance for the wintering of Anser anser and Mareca penelope; besides, it is an important national area for Mareca strepera, Anas crecca, Anas acuta, Spatula clypeata, Ardea alba, Platalea leucorodia, Phoenicopterus roseus, Recurvirostra avosetta, Pluvialis apricaria, Calidris alpina, Vanellus vanellus, Gallinago gallinago, Numenius arquata, Tringa erythropus and Tringa totanus. The protected area is an important breeding site under the Directive 2009/147/CE for species nesting in wetlands, fallows, dry and brackish pastures, shrub-like areas on rocky outcrops, woodland edges, partially flooded habitats and sandy littoral zones, as for example, with low number of pairs, Pandion haliaetus (1 pair), Burhinus oedicnemus (7-8 pairs), Charadrius alexandrinus (1 pair), Caprimulgus europaeus (25 pairs), Coracias garrulus (13-15 pairs), Lanius collurio (1-5 pairs), Lullula arborea (10 pairs) and Anthus campestris (6-7 pairs). In 2015, for the first time, Ardea cinerea started nesting in a heronry.
\end{abstract}

Key words: birds, check-list, conservation, Maremma Regional Park, ornithological relevance. 


\section{INTRODUZIONE}

Il Parco Naturale della Maremma, istituito con Legge della Regione Toscana n.65 del 5 giugno 1975, è un'area di importanza internazionale per la biodiversità. Ne costituisce una testimonianza fondamentale il Diploma Europeo assegnato dal Consiglio d'Europa nel 1992 e riconfermato vent'anni dopo per i meriti conseguiti con la sua gestione. In seguito, con Legge n.24 del 16 marzo 1994, l'area protetta ha assunto la denominazione di Parco Regionale e la figura giuridica di Ente Parco dotato di apposito statuto.

Secondo Parco istituito da una amministrazione regionale, dopo quello lombardo del Ticino nel 1974, il Parco Regionale della Maremma costituisce il punto di arrivo di iniziative, confronti, dibattiti e scontri protratti per molti anni (cfr. Guerrini, 1981). In una fase storica nella quale, già nei decenni precedenti, discutibili interventi compromettono in parte l'integrità ambientale della costa tirrenica, nel 1963 a Grosseto vede l'avvio un progetto di tutela del comprensorio dei Monti dell'Uccellina e della Palude della Trappola attraverso un convegno a cui partecipano le maggiori associazioni protezionistiche italiane e forze politiche locali. In Parlamento viene depositata una proposta di legge per l'istituzione di un Parco Nazionale e, quasi contestualmente per la stessa area geografica, di una Riserva Naturale che certificano il valore naturalistico del comprensorio (Società Botanica Italiana, 1971). Nel 1974, il Consorzio istituito tra i comuni di Grosseto, Magliano in Toscana, Orbetello e l'Amministrazione Provinciale di Grosseto per la gestione di una possibile area protetta, incarica la sezione regionale della Società Botanica Italiana a svolgere una ulteriore indagine conoscitiva del territorio finalizzata ad individuare eventuali nuove emergenze naturalistiche e suggerire la forma di tutela più opportuna per la quale, a conclusione, si formula la proposta di Parco Naturale (Arrigoni et al., 1977). Siamo ancora ben lontani dal poter fornire una rappresentazione adeguata delle diverse componenti biologiche segnalate nel territorio, con l'avifauna acquatica che comunque risulta di forte interesse per l'intensa attività venatoria esercitata in un ampio comprensorio al cui interno ricadono la Riserva dell'Alberese (in quegli anni gestita dall'Opera Nazionale Combattenti) e quella della Palude della Trappola (allora in massima parte di proprietà della famiglia Ponticelli). In questa circostanza non può che essere confermata quella che sin dall'inizio dell'area protetta risulta essere l'attrattiva prevalente dei ricercatori, indirizzati verso questo gruppo ed i rapaci (Allavena, 1975b; Lovari \& Martelli, 1977; Chelini, 1983), mentre molto tempo ancora occorre attendere prima di giungere ad un primo elenco commentato delle specie contattate (Giovacchini, 2006).

La grande varietà ambientale è uno dei caratteri più significativi del Parco Regionale della Maremma, con numerose tipologie vegetazionali che richiamano a complessi ecosistemici, atti più facilmente a procurare effetti positivi sull'ambiente e sulle stesse popolazioni di uccelli, così come al mantenimento degli equilibri nei vari ecosistemi. Quindi, grazie anche alle azioni intraprese per la gestione di questo territorio, l'area protetta depone da tempo nel rilevare il concetto fornito dalla presenza di una elevata ricchezza specifica (Giusti et al., 1993). Non è un caso che, anche per la numerosità dei contingenti di talune specie, i censimenti effettuati sull'avifauna acquatica svernante attribuiscono a questa zona un importante ruolo (Chelini, 1984; Arcamone et al., 2007; Zenatello et al., 2014). Tuttavia, per quanto al contrario le informazioni sull' avifauna risultino spesso non recenti o di scarso dettaglio, al fine di mantenere l'area protetta in un adeguato stato di conservazione, occorre anche disporre di uno strumento organico che riassuma le conoscenze sulle specie di uccelli del Parco (Andreella et al., 2008). Questa esigenza tiene conto, ad esempio, della necessità di garantire nel futuro una regolare verifica dei risultati raggiunti e legati alle conoscenze nella Rete Natura 2000 in applicazione alla Direttiva "Uccelli" 147/2009/CE (Nardelli et al., 2015).

Gli scopi di dettaglio del presente contributo sono quindi: - disporre di una solida base conoscitiva per intraprendere il successivo aggiornamento dell'inventario;

- fornire un quadro generale delle località più significative in termini di specie rinvenute nell'Allegato I della Direttiva "Uccelli" 147/2009/CE o come SPEC "Species of European Conservation Concern" (Staneva \& Burfield, 2017);

- individuare azioni tese a garantire una gestione esclusivamente conservativa di taluni ambienti, in particolar modo con riferimento a quelle specie nidificanti e svernanti comprese nelle stesse liste di attenzione o segnalate da ISPRA Istituto Superiore per la Protezione e la Ricerca Ambientale.

\section{AREA DI STUDIO}

Il Parco Regionale della Maremma, con una superficie di 8.902 ha, è collocato nella parte centrale della provincia di Grosseto, Toscana meridionale, interessando i comuni di Grosseto, Magliano in Toscana e Orbetello. L'area di studio è delimitata: a nord dall'abitato di Principina Mare e, per circa $4 \mathrm{~km}$, dalla strada provinciale "Trappola" sino alla strada di accesso per la Tenuta Trappola; ad est dalla strada provinciale "Alberese" in corrispondenza dell'incrocio con il canale Essiccatore Principale e poi, da Alberese Scalo, dalla strada statale Aurelia sino a Fonteblanda con una limitata appendice nell'area della Fattoria del Collecchio; a ovest, lungo circa $25 \mathrm{~km}$ di costa, dal Mare Tirreno; infine, a sud, dalla frazione di Talamone (Fig. 1).

Questo territorio include 6 siti facenti parte della Rete Natura 2000 classificati come ZSC - Zona Speciale di Conservazione (Direttiva "Habitat" 92/43/CE) e/o come ZPS - Zona di Protezione Speciale (Direttiva "Uccelli" 2009/147/CE) (Tab. 1). Nel 2013, il Ministero dell'Ambiente e della Tutela del Territorio e del Mare ha designato l'area "Palude della Trappola - Foce dell'Ombrone" (536 ha) quale zona umida di importanza internazionale ai sensi della Convenzione di Ramsar. Infine, BirdLife International ha individuato nel territorio del Parco Regionale della Maremma una Important Bird Area (IBA098 “Monti dell'Uccellina, Stagni della Trappola e Bocca d'Ombrone"), comprendente anche una fascia marina di protezione di $2 \mathrm{~km}$ (Gariboldi et al., 2000).

Il regime pluviometrico dell'area protetta presenta due picchi: uno principale, in autunno, ed uno secondario, in inverno. La stagione meno piovosa è l'estate, caratterizzata da un prolungato periodo d'aridità, alte temperature e ridotta escursione termica giornaliera. La variabilità annuale è certamente più accentuata rispetto alle precipitazioni (da $301 \mathrm{a} 1.063 \mathrm{~mm}$ ) che non alle temperature (AA.VV., 2000). 


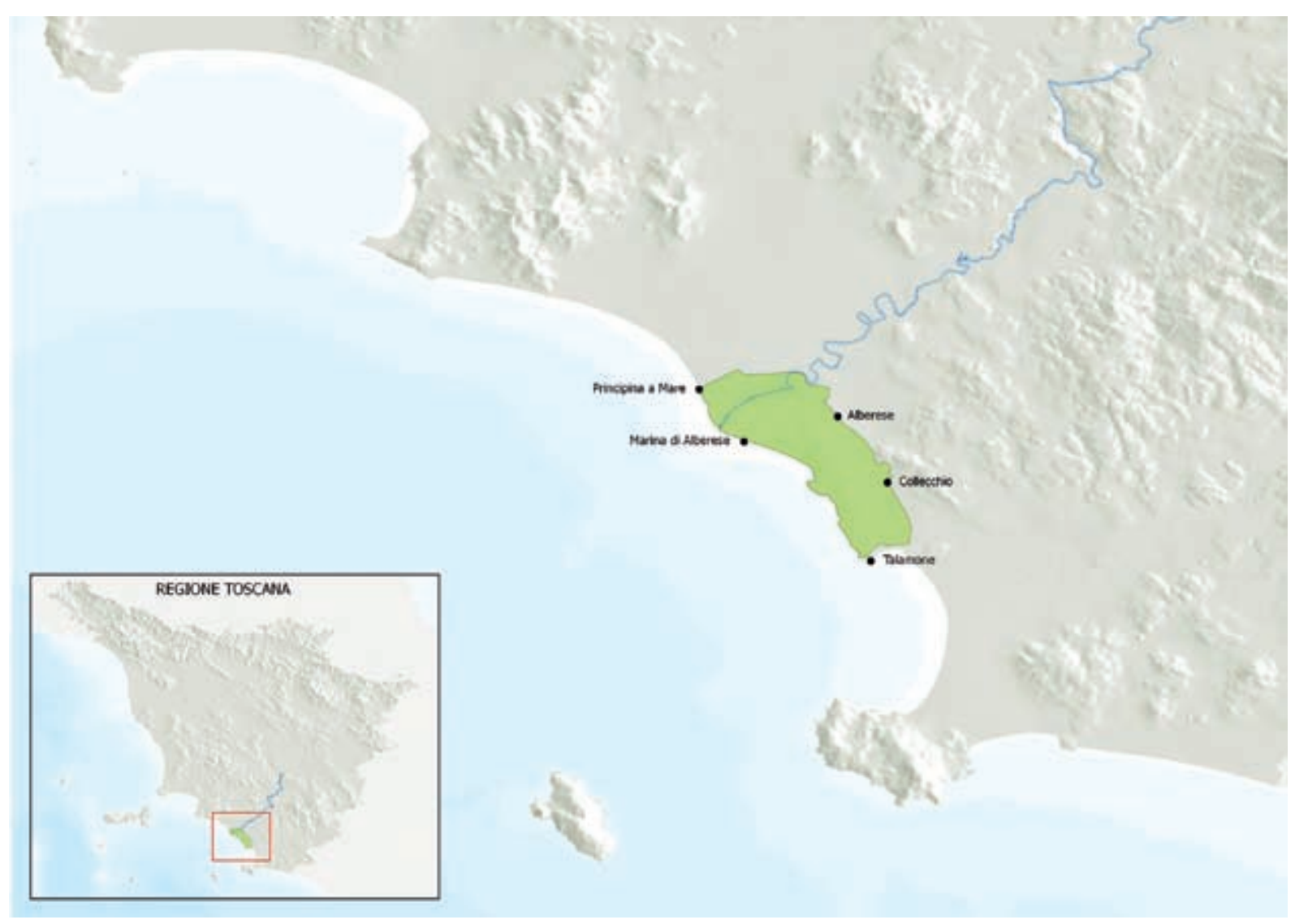

Fig. 1 - Localizzazione del Parco Regionale della Maremma a livello locale e regionale. / Area and location of Maremma Regional Park at regional scale (fonte: dati Regione Toscana. / Data from Regione Toscana's government).

Tab. 1 - Siti della Rete Natura 2000 nel Parco Regionale della Maremma. / Natura 2000 sites in Maremma Regional Park.

\begin{tabular}{|l|c|l|c|}
\hline ZSC/ZPS & Codice Natura 2000 & Denominazione & Superficie (ha) \\
\hline ZPS & IT51A0013 & Padule della Trappola, Bocca d'Ombrone & 489 \\
\hline ZSC/ZPS & IT51A0014 & Pineta Granducale dell'Uccellina & 626 \\
\hline ZSC/ZPS & IT51A0015 & Dune costiere del Parco dell'Uccellina & 158 \\
\hline ZSC/ZPS & IT51A0016 & Monti dell'Uccellina & 4441 \\
\hline ZPS & IT51A0036 & Pianure del Parco della Maremma & 3303 \\
\hline ZSC & IT51A0039 & Padule della Trappola, Bocca d'Ombrone & 495 \\
\hline
\end{tabular}

Nell'area protetta, relativamente alla morfologia, si possono distinguere tre tipologie paesaggistiche, variamente interessate da strade e abitati (Arrigoni, 2003; Colombini \& Chelazzi, 2007) e precisamente:

- la successione da Alberese a Talamone delle colline dell'Uccellina (i cosiddetti "Monti dell'Uccellina"), lungo la direttrice nord-sud sino alla quota di $417 \mathrm{~m} \mathrm{s.1.m.} \mathrm{del}$ Poggio Lecci, contraddistinte sul versante occidentale da un'alta costa rocciosa a falesia $\mathrm{o}$, più raramente, sabbiosa, e formate prevalentemente da calcare cavernoso, calcare massiccio, arenarie e argilloscisti del Verrucano (Figg. 2-3). Questi tratti geologici concordano con la quasi assoluta mancanza di acqua in superficie e con la definizione di sistema collinare areico data da Guerrini (1981). Le condizioni macro e mesoclimatiche caldo-aride determinano lo sviluppo di formazioni vegetali xerofitiche con sclerofille mediterranee e permettono di individuare due versanti ecologicamente differenziati. Nel sistema collinare possiamo infatti distinguere un'area caratterizzata da substrato calcareo, con vegetazione calcicola, e una caratterizzata da substrato siliceo del Verrucano, con vegetazione silicicola, in cui l'importante fattore di diversificazione costituito dall'esposizione sottolinea ulteriormente le differenze tra le formazioni vegetali mediterranee sempreverdi e quelle prossime del settore planiziario poggiante su dune e alluvioni recenti; 


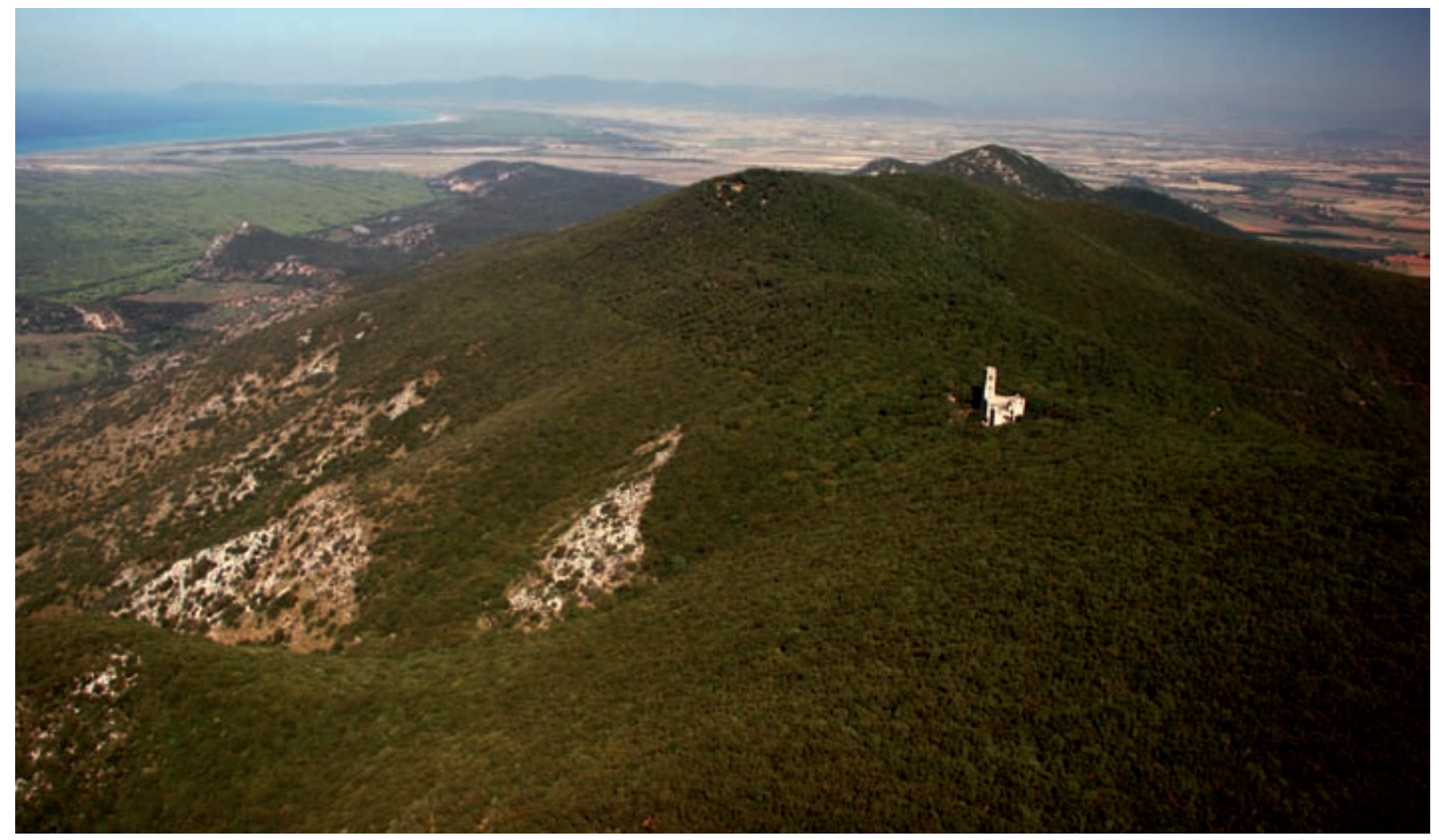

Fig. 2 - I Monti dell'Uccellina e l'Abbazia di San Rabano. / The Uccellina's mountains and San Rabano's abbey (Foto / Photo Giuseppe Anselmi).

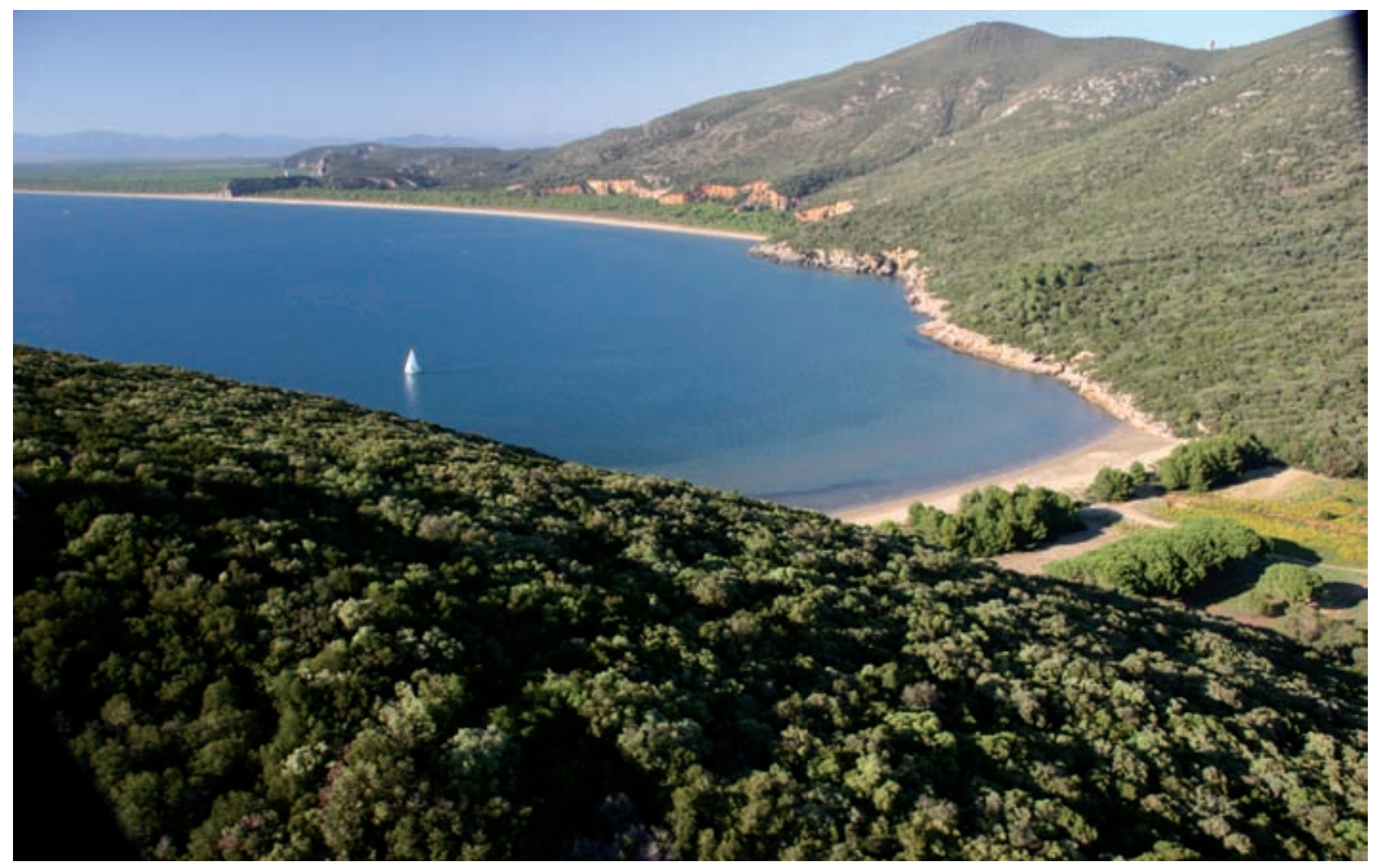

Fig. 3 - Cala di Forno. / Cala di Forno locality (Foto / Photo Giuseppe Anselmi). 
- l'area planiziaria dell'ultimo tratto del fiume Ombrone con il sistema spiaggia-duna e i limitrofi specchi d'acqua temporanei o permanenti (tra i primi le Saline di San Paolo, mentre tra i secondi i "chiari o bozzi" propriamente detti Stagni della Trappola) di recente formazione e soggetti ad un certo dinamismo della linea di costa per l'intensa deposizione di materiali alluvionali, specialmente nei pressi della foce deltizia che, scarsamente influenzata dall'effetto delle maree, è un luogo di particolare valore ornitologico e naturalistico (Figg. 4-6);

- l'area planiziaria agricola più interna; tra tutte le aree considerate, questa è quella che risulta sottoposta maggiormente a forme di utilizzo umano, con pascoli e allevamenti estensivi di bovini ed equini nella Tenuta di Torre

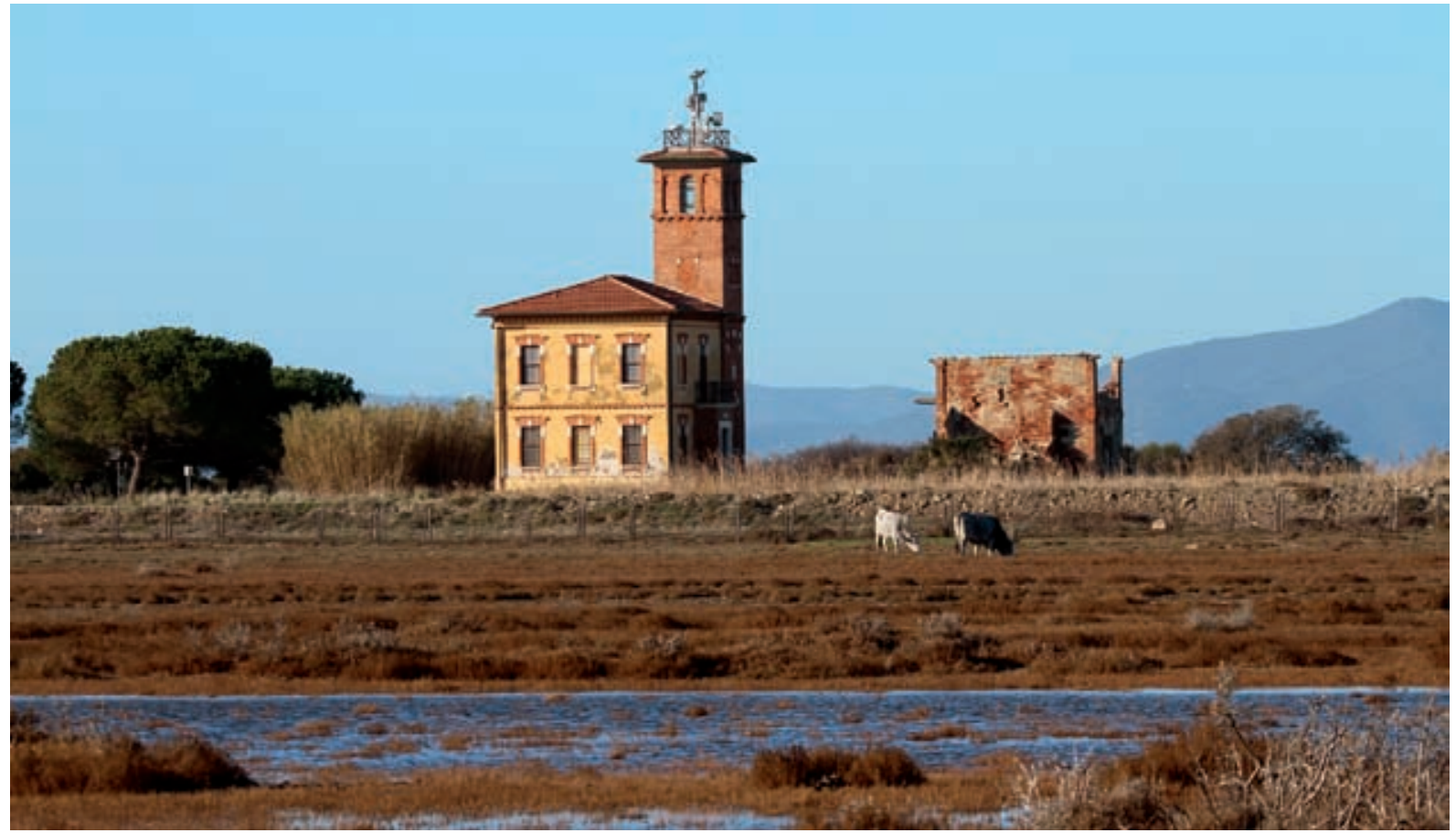

Fig. 4 - Saline di San Paolo e Casello Idraulico a Bocca d'Ombrone. / San Paolo salt flats and old point control of the water at Ombrone river mouth (Foto / Photo Giovanni Cappelli).

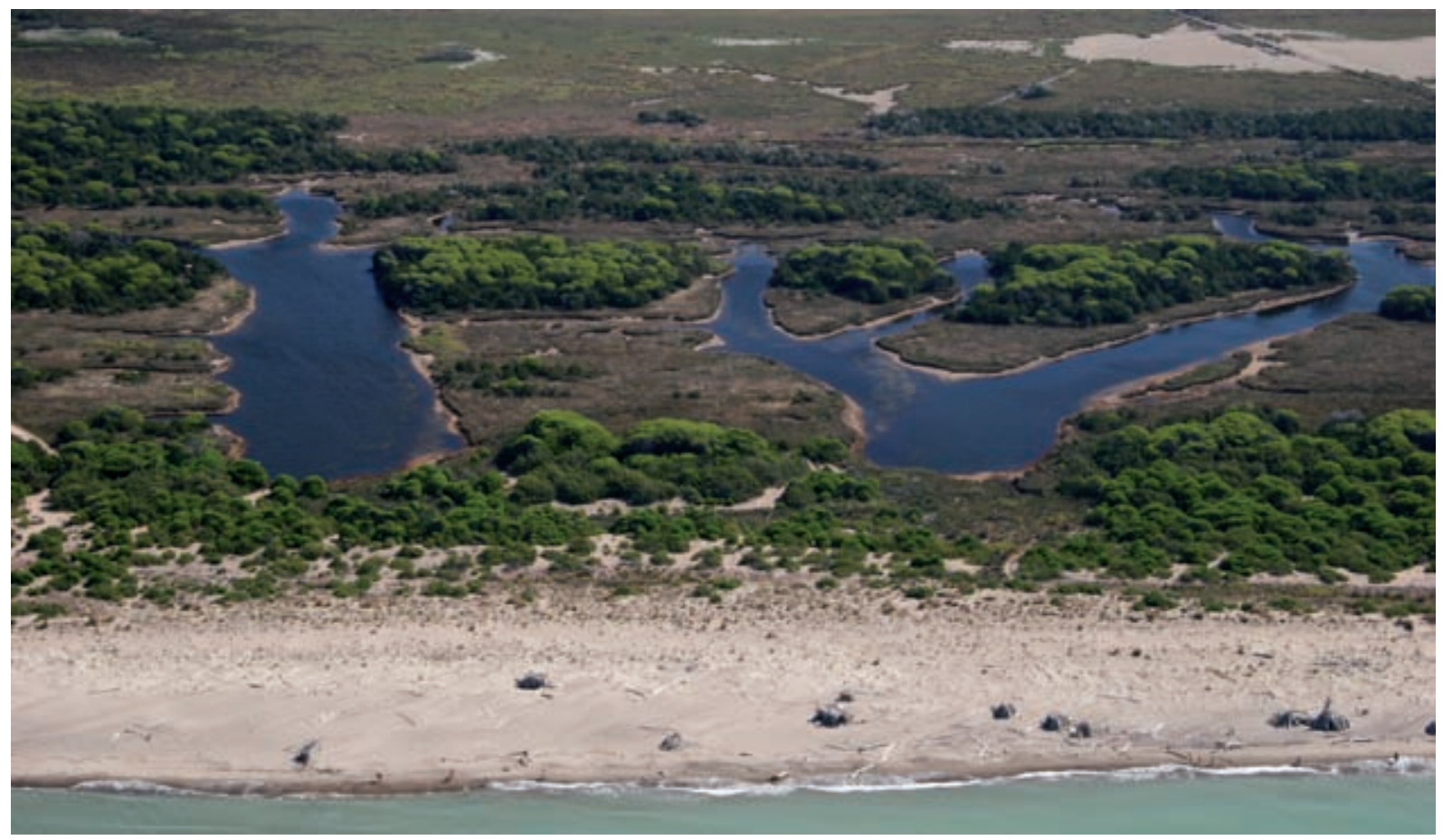

Fig. 5 - Chiari del Porciatti e Palude della Trappola in estate. / Porciatti's ponds and Trappola's marsh in summer season (Foto / Photo Giuseppe Anselmi). 


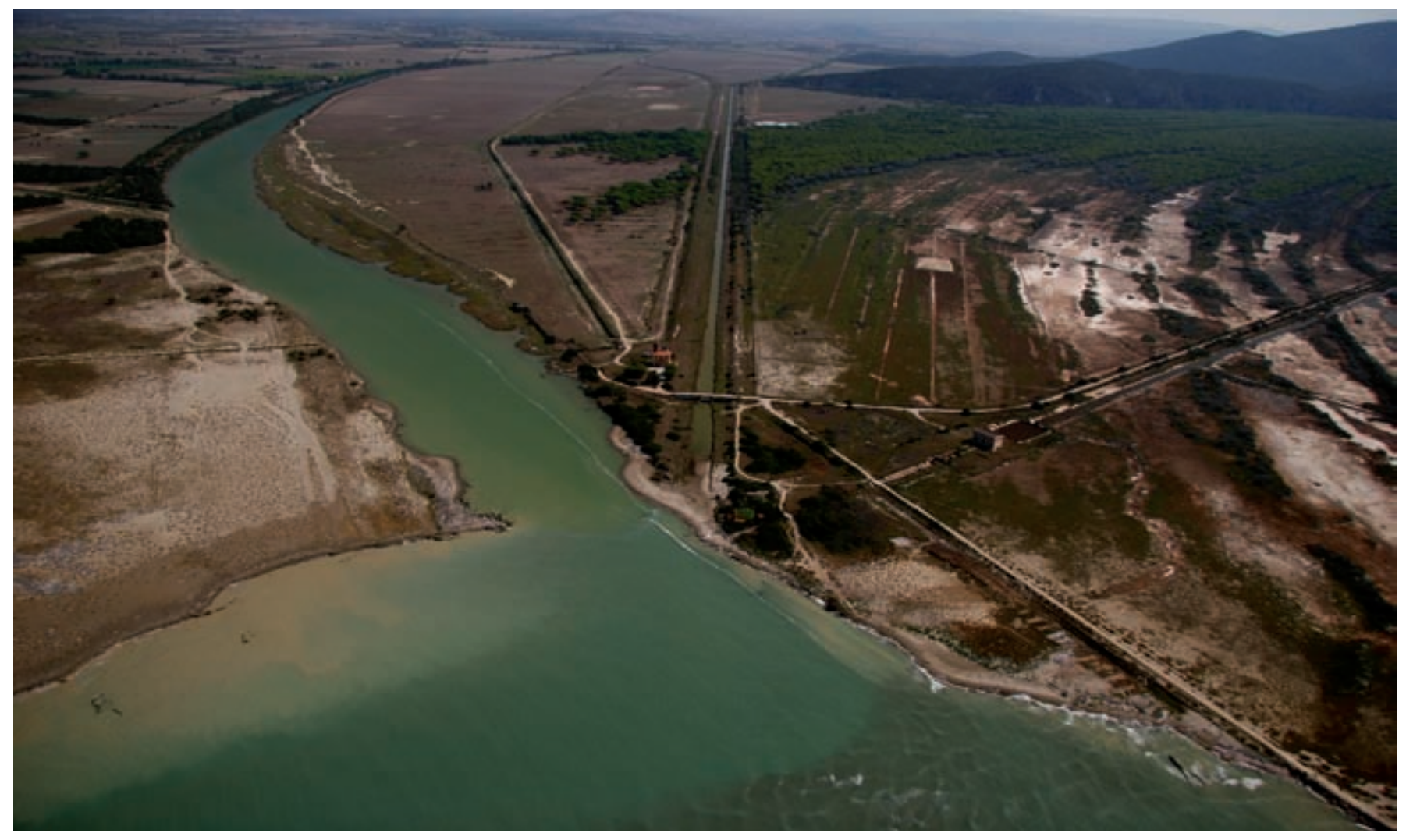

Fig. 6 - Foce del fiume Ombrone e pianure costiere del Parco della Maremma. / Ombrone river mouth and protected area coastal plains (Foto / Photo Giuseppe Anselmi).

Trappola, nell'Azienda delle Terre Regionali Toscane di Alberese, sino ai margini di una vasta pianura sabbiosa sulla sinistra idrografica del fiume Ombrone occupata dalla "Pineta Granducale" a Pinus pinea (600 ha). La realizzazione di una vasta rete idraulica, maggiormente sviluppata all'inizio del XX secolo per la bonifica dei terreni acquitrinosi, costituisce oggi l'aspetto più evidente delle trasformazioni operate in questo territorio (Figg. 7-10).

La grande varietà ambientale è dunque uno dei caratteri più significativi dell'area protetta; richiamandoci alle numerose tipologie vegetazionali riconosciute si possono evidenziare i seguenti complessi ecosistemici (Arrigoni et al., 1985):

- l'ecosistema forestale delle colline dell'Uccellina, con Quercus ilex prevalente che forma il $40 \%$ delle ceppaie e quasi tutte le matricine. Questo ecosistema si presenta con aspetti termofili e xerofili nelle esposizioni occidentali e meridionali, mesofili con partecipazione di latifoglie decidue nelle esposizioni nord-orientali;

- l'ecosistema palustre, differenziato notevolmente secondo il grado di salinità dell'acqua. Nei lamineti di acqua dolce o debolmente salmastra si sviluppano giuncheti, cariceti e scirpeti. Quando l'acqua ha una concentrazione di cloruro di sodio maggiore del $2 \%$ si sviluppano le associazioni alofile dei salicornieti;

- l'ecosistema delle dune, formatosi sui cordoni dunali che, in epoca storica, si sono depositati nella piana dell'Ombrone, verso la foce. Questo sistema, per sua natura, è frequentemente intercalato con depressioni stagionali umide. Gli stessi cordoni dunali sono stati incisi dai bracci fluviali di un antico sistema deltizio dell'Ombrone, di cui rimangono alcuni stagni, i cosiddetti chiari. Questo ecosistema è molto variabile a causa dei fenomeni contrapposti del trasporto fluviale di sedimenti che determina la formazione di nuovi cordoni dunali e dell'erosione marina della costa. La vegetazione naturale si differenzia, dal mare verso l'interno, in associazioni che vanno dal tipo erbaceo, a quelli arbustivo ed arboreo. Nella maggior parte della piana dell'Ombrone la vegetazione psammofila naturale è stata sostituita dalla selvicoltura, con fustaie alte 12-20 $\mathrm{m}$ di Pinus pinea e di Pinus pinaster, nel cui sottobosco spontaneo crescono sclerofille sempreverdi (Figg. 11-12);

- gli ecosistemi erbaceo-arbustivi delle garighe e degli incolti, con praterie e arbusteti spontanei conseguenza di precedenti interventi dell'uomo sulla comunità vegetale climacica. Alcuni di essi, come le garighe, derivano da incendi o dall'antica presenza di coltivazioni. Se l'azione di gestione dell'uomo non è costante, questi aspetti vegetazionali tendono ad evolvere naturalmente verso la macchia e il bosco. Questo tipo ambientale è caratterizzato da una grande ricchezza floristica con un numero rilevante di specie endemiche o con una distribuzione localizzata nel Paese;

- l'ecosistema agricolo, con le colture agrarie che formano una fascia più o meno continua rispetto alle aree naturali con vegetazione spontanea. Il sistema agrario è un elemento di possibile disturbo per alcuni scopi del Parco, soprattutto se l'ordinamento colturale prevede il ricorso a pratiche agronomiche con uso di antiparassitari e concimi di sintesi (Fig. 13). 


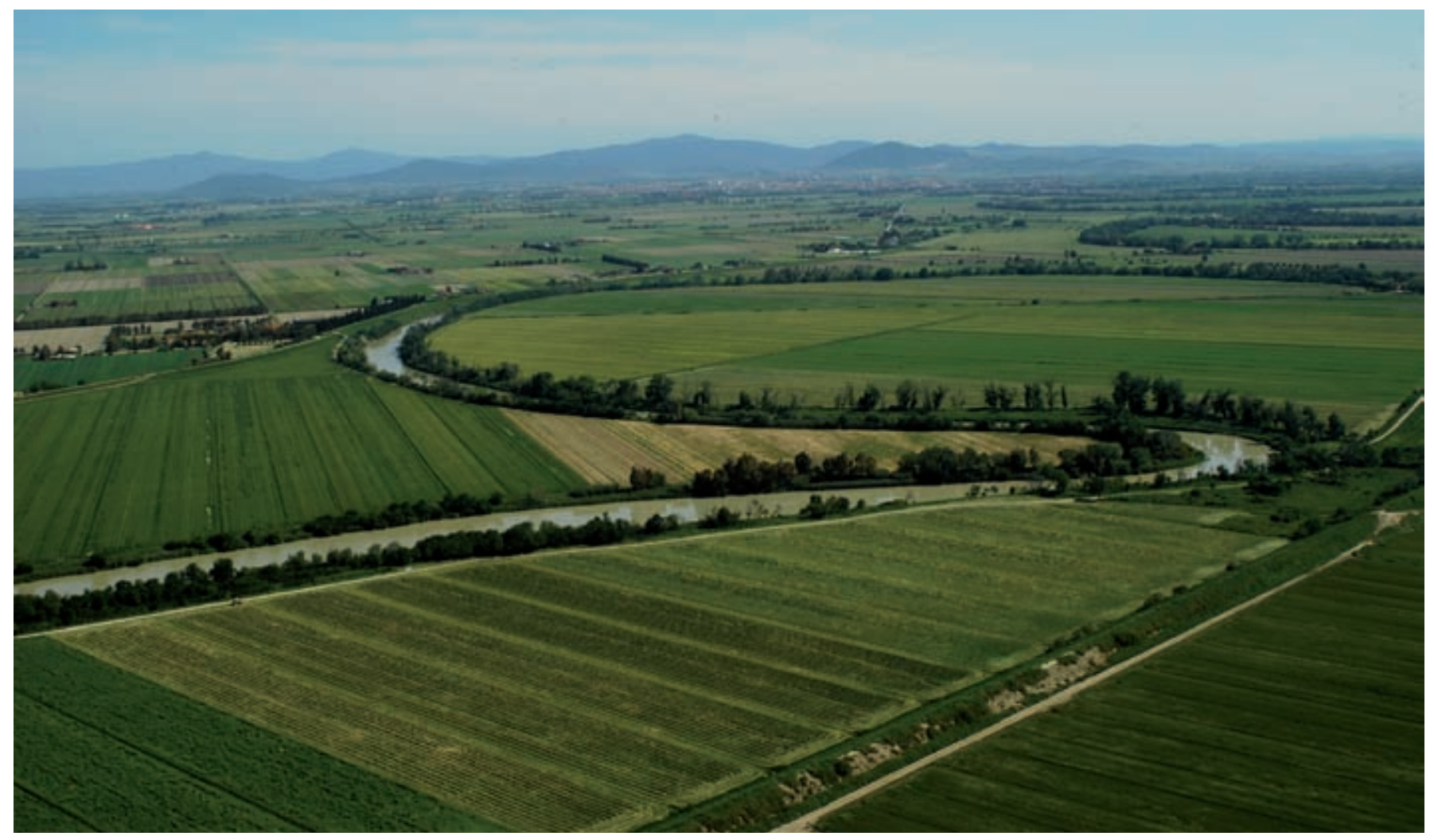

Fig. 7 - Le aree agricole di Pian di Barca, San Mamiliano e Spolverino. / Agricultural areas of Pian di Barca, San Mamiliano and Spolverino (Foto / Photo Giuseppe Anselmi).

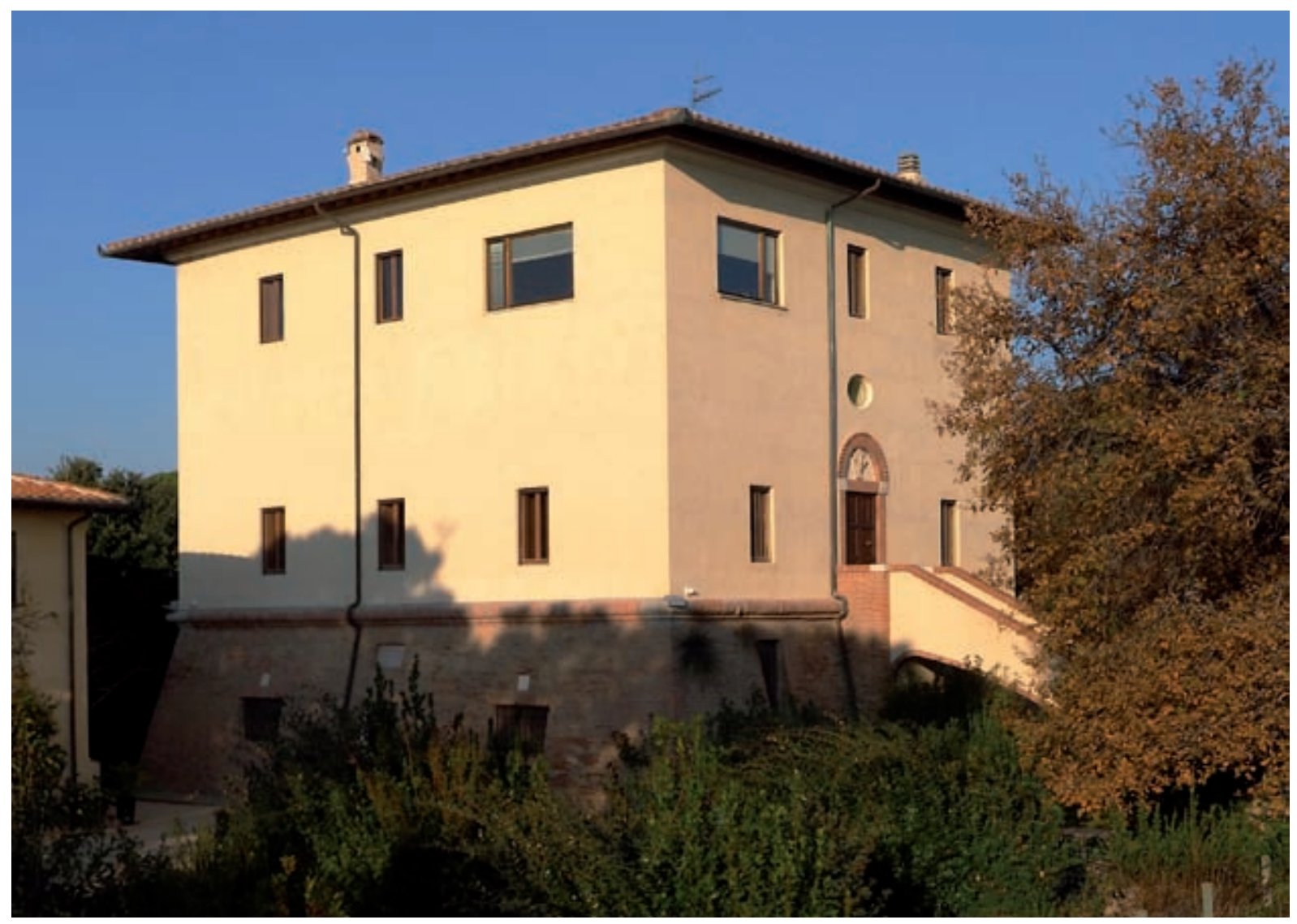

Fig. 8 - Torre Trappola recentemente restaurata / Torre Trappola recently restored (Foto / Photo Stefano Laurenti). 


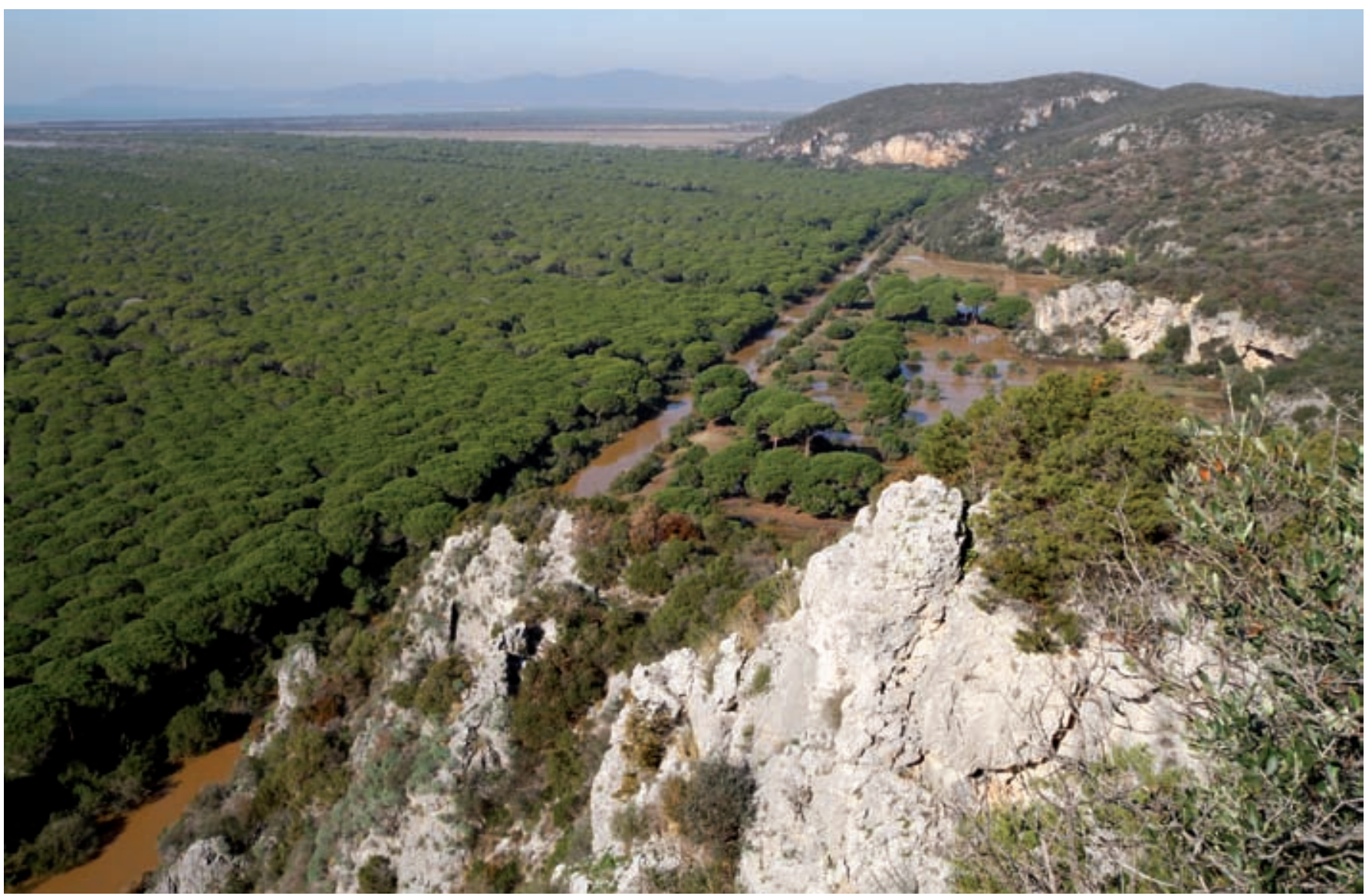

Fig. 9 - La Pineta Granducale di Alberese osservata da Castel Marino. / Granducale pinewood in Alberese viewed from Castel Marino (Foto / Photo Giuseppe Anselmi).

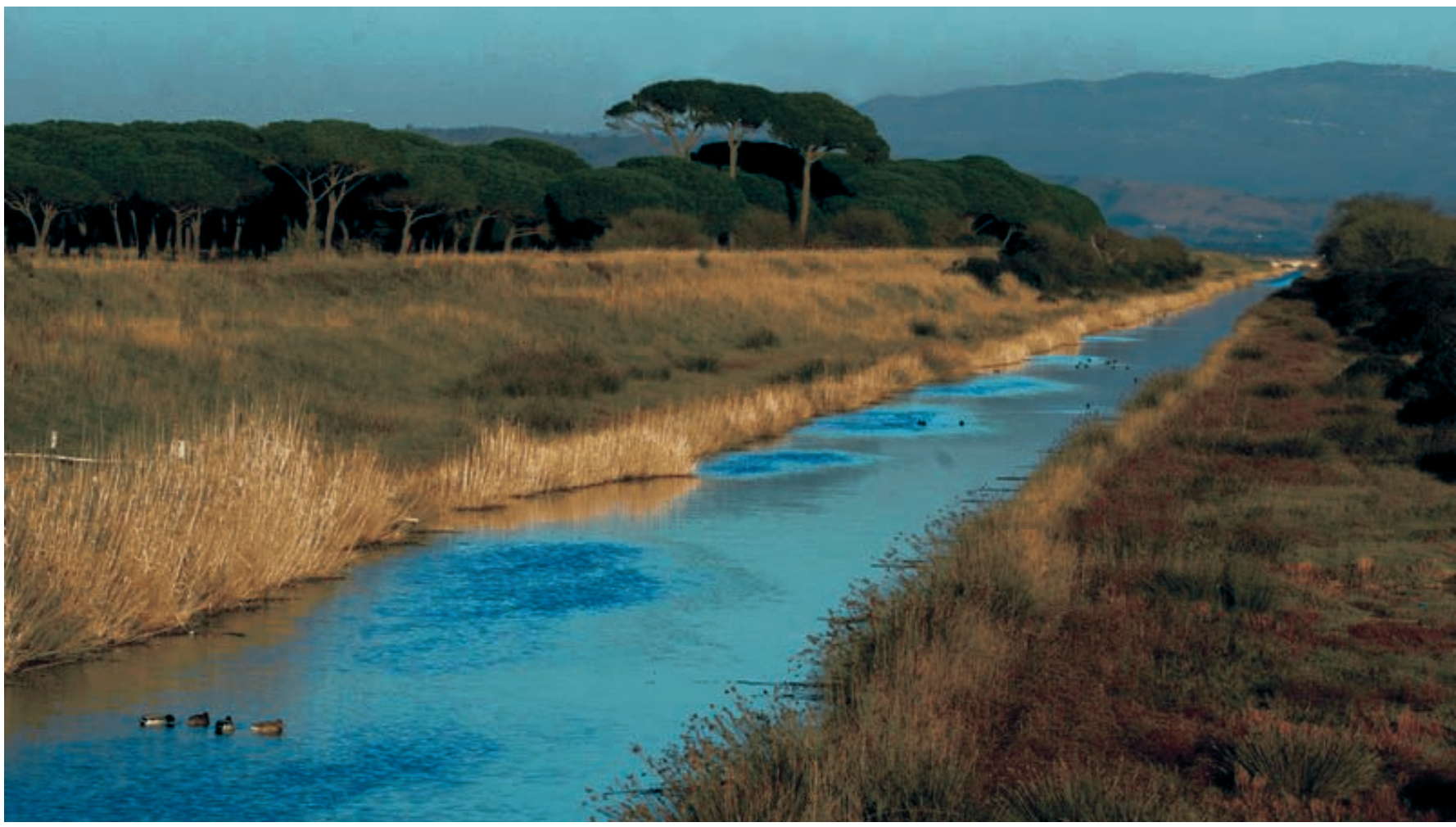

Fig. 10 - Il Canale Essiccatore. / The Essiccatore channel (Foto / Photo Giuseppe Anselmi). 


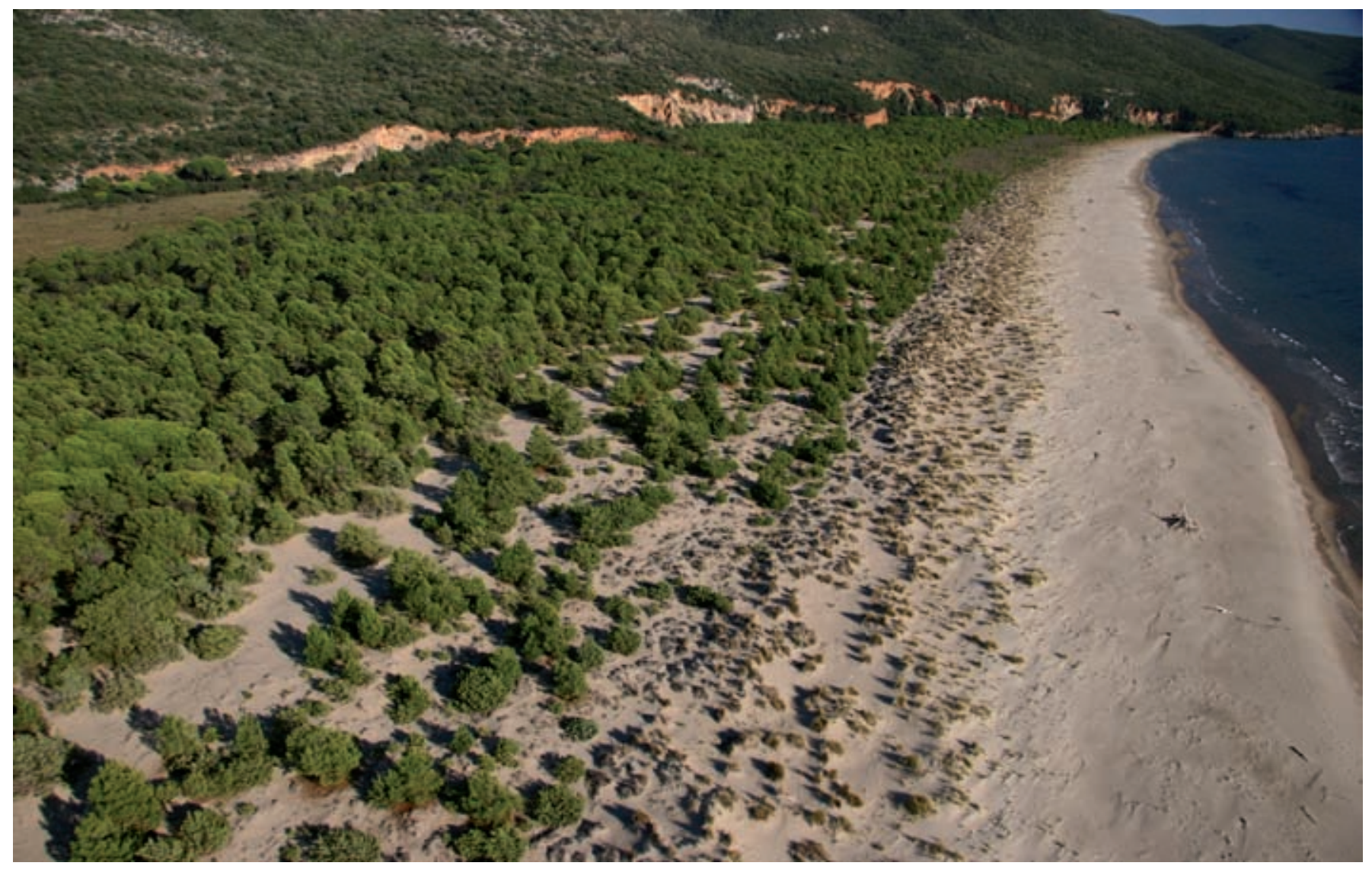

Fig. 11 - Spiaggia di Cala Rossa o Cala Francese. / Cala Rossa or Cala Francese beach (Foto / Photo Giuseppe Anselmi).

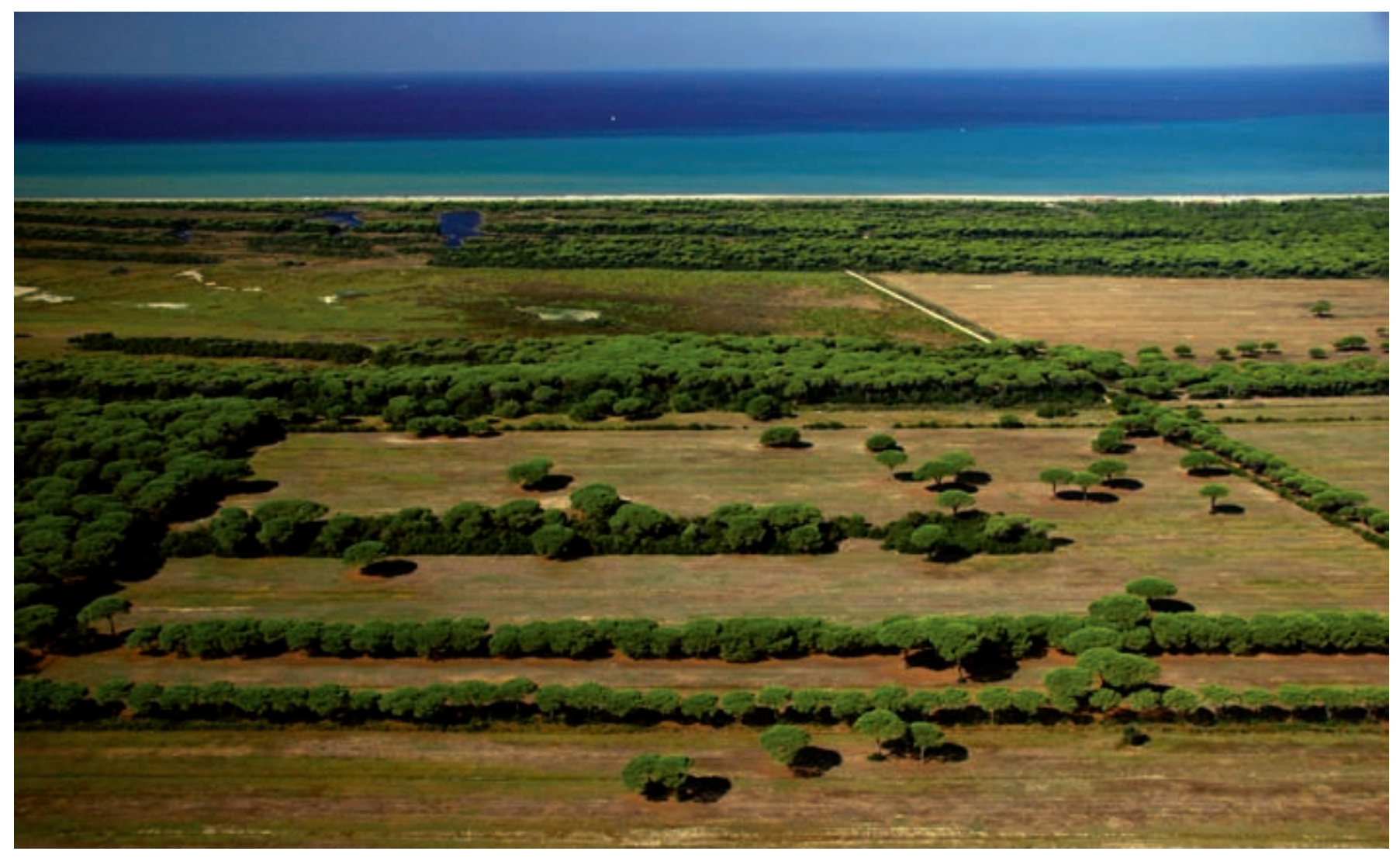

Fig. 12 - Pineta e serrata della Tenuta di San Carlo. / San Carlo pinewood (Foto / Photo Giuseppe Anselmi). 


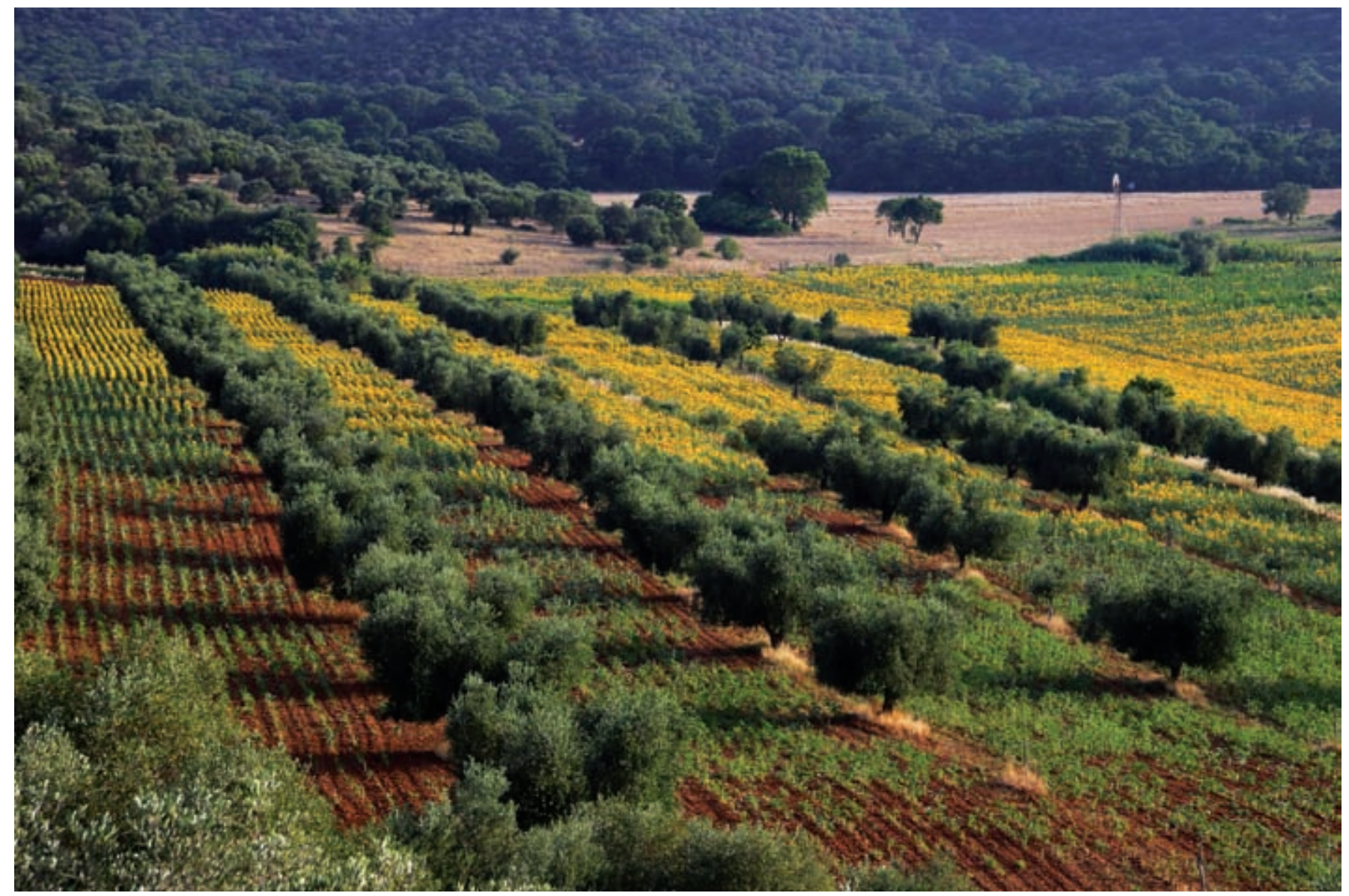

Fig. 13 - Coltivazioni tradizionali nella zona di Vaccareccia. / Traditional crops near Vaccareccia (Foto / Photo Giuseppe Anselmi).

\section{MATERIALI E METODI}

Il presente contributo riassume le conoscenze relative all'avifauna del Parco Regionale della Maremma ottenuto analizzando la bibliografia e utilizzando banche dati, ricerche sul campo condotte, in particolar modo dall'autore, anche con metodologie standardizzate (cfr. Bibby et al., 2000), lungo tutto l'anno solare a partire dal 1986 . Ne è un esempio l'attività realizzata nel periodo riproduttivo 2000-2004 nell'ambito del Progetto “Monitoraggio Italiano Ornitologico MITO2000" (per un totale di 91 punti di ascolto nell'area protetta).

Inoltre, parte dei dati derivano dalla ricerca su fonti bibliografiche (periodici e monografie) e dalla consultazione di banche dati, mailing-list moderate, piani di gestione, tesi di laurea e fonti archivistiche pubbliche e private. In particolare sono stati utilizzati:

- articoli o note scientifiche contenenti osservazioni realizzate nel Parco a partire dal giugno 1975;

- check-list degli uccelli del Parco Regionale della Maremma, aggiornata al 15 febbraio 2005 (Giovacchini, 2006);

- atlante degli uccelli svernanti in provincia di Grosseto (Corsi \& Giovacchini, 1995a);

- atlante degli uccelli nidificanti e svernanti in Toscana (Tellini Florenzano et al., 1997);

- censimenti degli uccelli acquatici svernanti secondo gli standard IWC (International Waterfowl Census) rea- lizzati dal Centro Ornitologico Toscano (COT) per conto della Regione Toscana, Direzione Agricoltura e Sviluppo Rurale (F. Pezzo, com. pers.; archivio Ente Parco);

- resoconti ornitologici per la Toscana (Arcamone \& Tellini, 1985; 1986; 1987; 1988; 1991-1992; Arcamone \& Barbagli, 1995-1996; Arcamone \& Puglisi, 2006; 2008);

- volume "Lo svernamento degli uccelli acquatici in Toscana: 1984-2006" (Arcamone et al., 2007);

- volume "Storia Naturale della Toscana Meridionale" (Giusti et al., 1993);

- volume "Il Parco della Maremma. Storia e natura" (Ciuffoletti \& Guerrini, 1989);

- Banca Dati Gruppo Ornitologico Maremmano (GOM) - Studi Naturalistici "A. Ademollo";

- piattaforma online Ornitho.it (www.ornitho.it);

- mailing list European Bird Net Italia (EBN-Italia) e GOM-List.

- volume "Fauna dell'Etruria Settentrionale" (Farina, 1977);

- volume "Le zone umide nei comprensori di bonifica della Toscana, del Lazio, dell'Umbria e della Sardegna" (Chelini, 1983);

- volume "I rapaci nel Parco Naturale della Maremma" (Martelli, 1983);

- volume "Il Parco Regionale della Maremma e il suo territorio" (Scapini \& Nardi, 2007); 
- volume "Guida ai Rapaci diurni del Parco Regionale della Maremma" (Morimando et al., 2008);

- volume "L'Aquila dei serpenti" (Petretti, 2008);

- volume "La protezione della natura in Toscana: Siti di Importanza Regionale e fauna vertebrata nella provincia di Grosseto" (Giovacchini \& Stefanini, 2008);

- Piani di Gestione del SIC/SIR IT51A0039 [SIR 113 e A113(ZPS)] Palude della Trappola e Bocca d'Ombrone; IT51A0014 [SIR 114] Pineta Granducale dell'Uccellina; IT51A0015 [SIR 115] Dune costiere del Parco dell'Uccellina (Sforzi, 2013);

- tesi di diploma universitario in tecnica faunistica; tesi di laurea sperimentali (corsi di laurea con vecchio ordinamento o specialistiche), realizzate su Airone guardabuoi, Cormorano, Falco pescatore, Piovanello pancianera, Ghiandaia marina, Falconiformi e Ghiandaia, depositate presso la sede del Parco;

- manoscritto sull'avifauna della Palude della Trappola negli anni 1978-1984, anch'esso presente presso la sede del Parco (M. Pani, ined.);

- archivio Progetto WWF-Provincia di Grosseto per la tutela dei siti riproduttivi di Albanella minore nel periodo 1998-2004 (F. Farsi, ined.).

Dal punto di vista della definizione dell'area considerata ai fini dell'inclusione/esclusione di una specie dal censimento, sono state considerate attribuibili al territorio del Parco anche le specie osservate sul mare sino a circa un miglio nautico dalla costa e, per via della contiguità ecologica, quelle osservate nelle Risaie di Principina e nella cava dismessa della Voltina situate a $30-2100 \mathrm{~m}$ in linea d'aria dall'area protetta.

L'ambito temporale di riferimento va dall'anno di costituzione dell'area protetta (1975) fino al 31/12/2018. Scopo del presente lavoro è quello di fornire uno strumento organico che riassuma le conoscenze sulle specie di uccelli del Parco e che possa essere di riferimento per successive azioni di monitoraggio e conservazione.

\section{RISULTATI}

Di seguito viene fornito l'elenco degli uccelli del Parco Regionale della Maremma in cui, per la nomenclatura italiana e scientifica e l'ordine sistematico, ci si è riferiti alla più recente IOC World Bird List (v.8.2) (Gill \& Donsker, 2018). Per la presenza di specie alloctone, rilasci di specie autoctone e casi che possano indurre dubbi sull'effettiva presenza o sull'origine di alcune specie, si rimanda a Baccetti et al. (2014).

Nella parte iniziale del testo predisposto per ogni specie, sono date informazioni sintetiche sul suo status e sulla tendenza come nidificante in Europa, sulla sua ecologia e sulla sua fenologia nell'area protetta (Brichetti \& Fracasso, 2003; 2004; 2006; 2007; 2008; 2010; 2011; 2013; 2015a; Peronace et al., 2012; Staneva \& Burfield, 2017).

Le categorie fenologiche utilizzate sono le seguenti:

S: Sedentary - Sedentaria

B: Breeding - Nidificante

M: Migratory - Migratrice (comprese le specie dispersive e quelle erratiche)

W: Wintering - Svernante (comprese le specie la cui presenza nel periodo invernale non risulta verosimilmente assimilabile ad un vero e proprio caso di svernamento)
A: Accidental - Accidentale (sino a 3 segnalazioni per la specie nel periodo 1975-2018)

ext: extinct - estinta

irr: irregular - irregolare

reg: regular - regolare

? = può seguire ogni simbolo e significa dubbio; " $\mathrm{M}$ reg ?" indica, per esempio, una possibile regolarizzazione delle comparse di una specie in precedenza considerata migratrice irregolare.

Per un maggiore approfondimento delle tipizzazioni delle categorie fenologiche si veda Fasola \& Brichetti (1984).

Altre abbreviazioni utilizzate nelle schede sono le seguenti:

ind. $=$ individuo $/ \mathrm{i}$

PRM = Parco Regionale della Maremma

COT $=$ Centro Ornitologico Toscano

GOM = Gruppo Ornitologico Maremmano

$\mathrm{CUA}=$ Censimento Uccelli Acquatici svernanti (dato raccolto in occasione del)

GIL = Gruppo Inanellamento Limicoli (S. Scebba, com. pers.)

$\mathrm{COI}=$ Commissione Ornitologica Italiana

ISPRA= Istituto Superiore per la Protezione e la Ricerca Ambientale

La cosiddetta Macrozona "Maremma Grossetana" (cod. GR 0200) raccoglie i dati ornitologici di più zone umide e di altre tipologie ambientali, con principale riferimento alla "Diaccia Botrona" (cod. GR 0202), "Bocca d'Ombrone" (cod. GR 0214) e "La Trappola" (cod. GR 0215), questi ultimi due all'interno del PRM.

I nomi degli autori di gran parte delle osservazioni sono così abbreviati (in ordine alfabetico): Giuseppe Anselmi (GA), Pietro Giovacchini (PG), Michele Lamberti (ML), Stefano Laurenti (SL), Luca Passalacqua (LP).

\section{ELENCO SISTEMATICO}

\section{ANSERIFORMES}

\section{Anatidae}

Oca colombaccio Branta bernicla A-1

Accidentale. 1 ind. il 06/09/1981 alla foce del fiume Ombrone (R. Nardi in Arcamone \& Brichetti, 1995).

\section{Oca del Canada Branta canadensis A-1}

Accidentale. Specie precedentemente non rinvenuta (Giovacchini, 2006). Segnalato 1 ind. il 19/01/2014 nella Palude della Trappola (fonte: COT, archivio PRM). Dubbia provenienza (Andreotti et al., 2001; Baccetti et al., 2014).

Oca facciabianca Branta leucopsis M irr, W irr

In Europa, è specie nidificante in incremento (Staneva $\&$ Burfield, 2017). Dopo la stagione riproduttiva viene osservata più facilmente in vasti coltivi su terreni di bonifica (Brichetti \& Fracasso, 2003). Nel PRM, è specie precedentemente non rinvenuta (Giovacchini, 2006). Osservata 
per la prima volta il 30/12/2006 con 1 ind. alle Macchiozze, comunque sino al 13/01/2007 (G. Paesani, A. Canci, A. Vezzani, B. Perroud e F. Perroud in EBN-Italia; CUA in Arcamone \& Puglisi, 2008; GA; PG). La specie viene anche riconfermata nelle stagioni autunno invernali 2008/09 (GA; PG) e 2012/13 (PG; D. Verducci, R. Gildi, B. e F. Perroud, G. Chiancianesi in EBN-Italia; ML; F. Cianchi; F. Pezzo; Fig. 14), rispettivamente con 1 ind. e 3 ind. ed ancora per l'intera stagione invernale 2013/14, con un massimo di 3 ind. (PG e SL; E. Occhiato, B. Perroud e F. Perroud in EBN-Italia). Segnalazione più precoce: 1 ind. il 11/11/2017 alle Macchiozze (PG), verosimilmente lo stesso il 03/12/2017 nelle risaie della Trappola (PG e SL). Rinvenuta più facilmente insieme ad Anser anser; i comportamenti rilevati negli anni paiono essere riconducibili a migratori selvatici.

\section{Oca selvatica Anser anser $\mathrm{M}$ reg, $\mathrm{W}$ reg}

In Europa, è specie nidificante in incremento (Staneva $\&$ Burfield, 2017). Nei periodi delle migrazioni e dello svernamento frequenta principalmente zone umide costiere d'acqua dolce o salmastra e vasti coltivi (Brichetti \& Fracasso, 2003). Nel PRM, è specie migratrice regolare, svernante di recente comparsa, in analogia con quanto verificato su scala nazionale (Arcamone \& Massi, 1987; Perco, 1992). Specialmente nell'ultimo decennio i contingenti del PRM, svernanti principalmente nei pascoli, seminativi e zone umide delle Macchiozze e della Palude della Trappola, risultano in evidente incremento numerico, con punte di oltre 3300-3700 ind. negli anni 2014 e 2016 (fonte: COT, archivio PRM). La rimessa notturna dei contingenti è attualmente ricompresa nella zona della Trappola, mentre nel passato interessava a volte anche il comprensorio di Alberese (Campo al Pino e Macchiozze, inverno 1990/91, PG). L'area di studio ricade nella Macrozona "Maremma Grossetana", sito di importanza internazionale per la specie (Zenatello et al., 2014). Individui marcati nei paesi di provenienza con collari alfa numerici, ed osservati al PRM specialmente in autunno-inverno nel periodo 1989/90-2015/16, mostrano l'assoluta rilevanza delle popolazioni dell'Europa orientale (96,4\%) e numerosi casi di fedeltà al sito (Giovacchini, 1995a; Baccetti, 2007; PG, banca dati ISPRA; Fig. 15).

\section{Oca granaiola della tundra Anser serrirostris M irr, W irr}

In Europa, è nidificante stabile (Staneva \& Burfield, 2017). Fortemente gregaria oltre la stagione riproduttiva, con le migrazioni e lo svernamento frequenta spazi molto aperti per l'alimentazione ed il riposo (Brichetti \& Fracasso, 2003). Specie svernante di comparsa scarsa e rara nel centro sud del Paese, per quanto comune in Toscana nei primi decenni del secolo scorso (Arcamone \& Massi, 1987; Zenatello et al., 2014). Nel PRM, le osservazioni di cui si ha certezza fanno riferimento ad alcuni inverni più recenti come le stagioni 2008/09 e 2010/11, unicamente con 3 ind. nei pressi della foce del fiume Ombrone (fonte: COT, archivio PRM) ed ancora in quella successiva, con 2 ind. alle Macchiozze almeno dal 27/11/2011 al 04/01/2012 (PG; D. Brendan, G. Paesani su Ornitho.it; Fig. 16). Altre scarse segnalazioni riguardano la migrazione primaverile: ad es. 1 ind. il 04/03/1996 (PG).

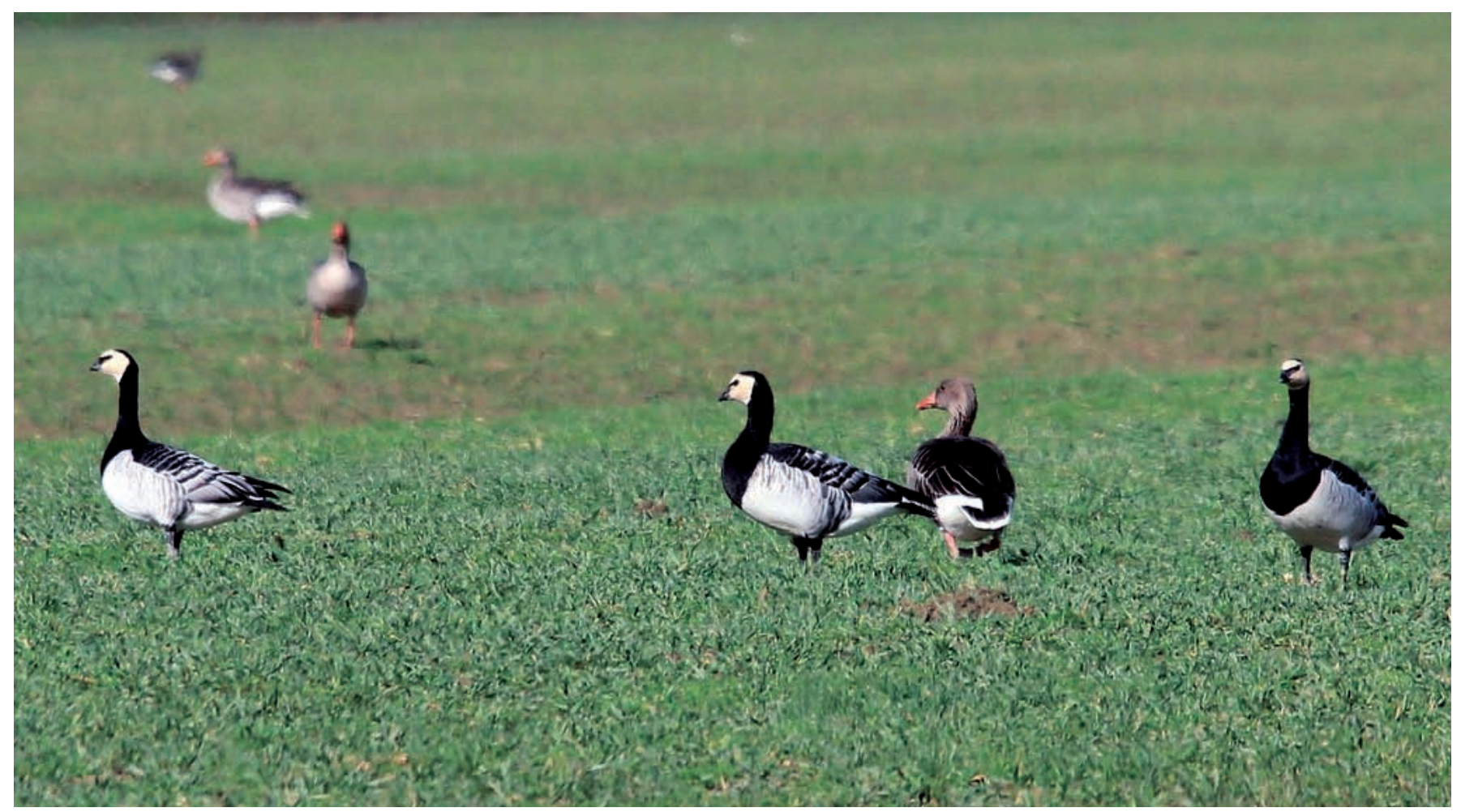

Fig. 14 - Oche facciabianca il 09/02/2013 alle Macchiozze. / Barnacle Gooses on the ${ }^{\text {th }}$ February 2013 at the Macchiozze (Foto / Photo Michele Lamberti). 


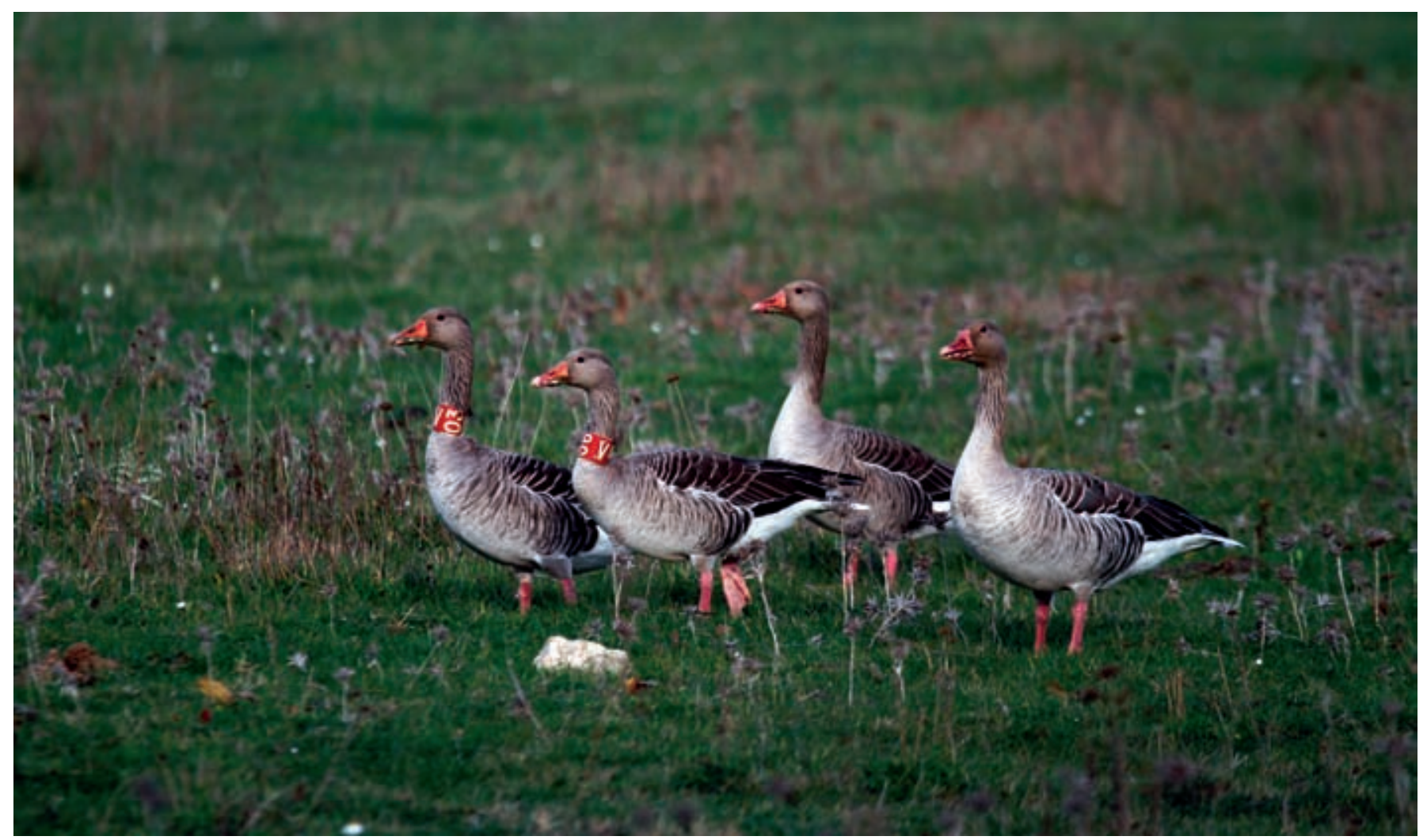

Fig. 15 - Oche selvatiche marcate nella repubblica Ceca. / Greylag Gooses marked in the Czech Republic (Foto / Photo Giuseppe Anselmi).

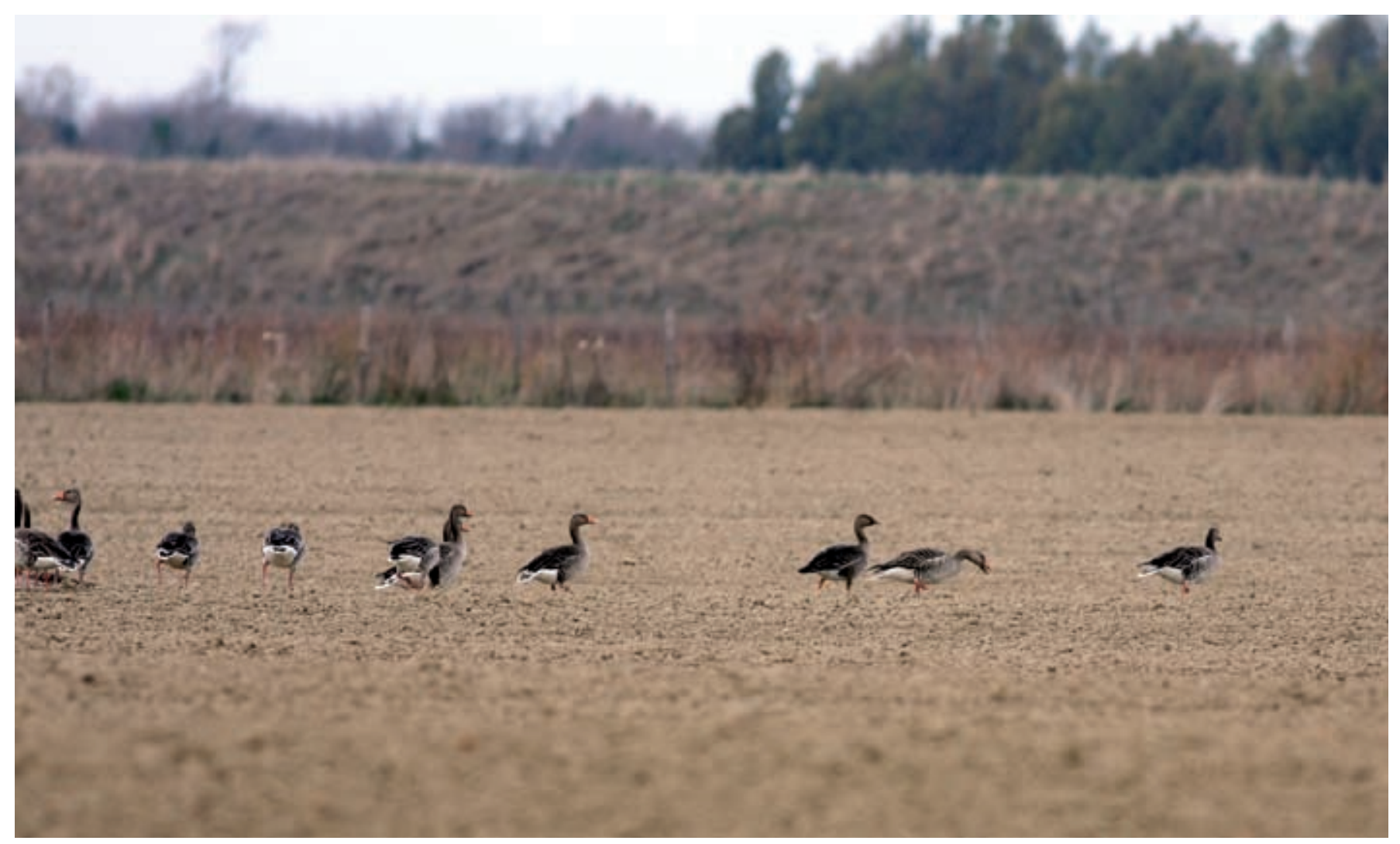

Fig. 16 - Oca granaiola e oche selvatiche il 10/12/2011 alle Macchiozze. / Bean Goose and Greylag Gooses on the 10th December 2011 at the Macchiozze (Foto / Photo Doe Brendan). 
Oca lombardella Anser albifrons $\mathrm{M}$ reg, W reg

In Europa, è nidificante stabile (Staneva \& Burfield, 2017). Durante le migrazioni e lo svernamento frequenta principalmente zone umide costiere d'acqua salmastra e vasti coltivi su terreni di bonifica (Brichetti \& Fracasso, 2003). Concentrazioni di rilevanza nazionale si trovano nel nord Italia, specialmente in provincia di Venezia (Zenatello et al., 2014), mentre la specie è decisamente più scarsa altrove, Toscana compresa dove, nei primi decenni del secolo scorso, rappresentava una frazione considerevole delle Anser sp. svernanti in questa regione (Arcamone \& Massi, 1987). Già dall'inverno 1980/81 sono comunque disponibili dati (M. Pani, ined.). Nel PRM, è svernante regolare dalla stagione 2008/09. Associata spesso in pastura e riposo con Anser anser. Massimo numero osservato per l'area protetta: 42 ind. il 13/01/2016 nell'area Macchiozze-Vaccareccia (ML e LP in GOM-List).

\section{Oca lombardella minore Anser erythropus A-2}

Accidentale. Segnalato 1 ind. il 15/01/1990 nei seminativi delle Macchiozze insieme ad un 1 ind. di Anser albifrons e 2 ind. di Anser anser (Arcamone et al., 2007). 1 ind. dal 31/12/2009 ad almeno il 19/01/2010 nell'area Macchiozze-Vaccareccia (A. Vezzani, G. Paesani in EBNItalia; GA; PG).

\section{Ibrido Oca del Canada Branta canadensis x Oca lom- bardella Anser albifrons}

1 ind. il 27/10/2001 alla foce del fiume Ombrone (PG; SL); 1 ind. il 08/12/2001 alla foce del fiume Ombrone (PG); 1 ind. il 12/12/2001 alle Macchiozze (PG).

Cigno reale Cygnus olor M irr, W irr, B (2010, tentativo)

In Europa, è specie nidificante in incremento (Staneva $\&$ Burfield, 2017). Con le migrazioni e lo svernamento frequenta zone umide naturali o artificiali, foci dei fiumi, laghi, paludi, con fondali generalmente bassi (Brichetti \& Fracasso, 2003). Nel PRM, è specie migratrice irregolare, svernante irregolare. L'origine selvatica degli individui può essere messa spesso in discussione per via della esistenza di una coppia introdotta, a metà degli anni ' 90 del secolo scorso, nel vicino Lago di Burano, ove si è poi riprodotta (Andreotti et al., 2001; Baccetti et al., 2014). La prima segnalazione è quella di 1 ind. il 26 e 27/12/1994 sul fiume Ombrone identificabile come un soggetto di origine forse selvatica, come lasciava supporre la cospicua distanza di fuga all'approssimarsi dell'osservatore (Giovacchini, 1995b). Più recentemente, ad es. 1 ind. osservato il 29/01/2006 alla foce del fiume Ombrone (PG), 2 immaturi il 5 e 6/07/2006 sempre alla foce del fiume Ombrone (GA e U. Bambi). In periodo riproduttivo 2010, alla foce del fiume Ombrone, è stato accertato il tentativo di nidificazione di 1 coppia, poi fallito, con un contingente allora composto da un massimo di 7 individui (GA). Nel corso dell'intero 2015 sono numerose le osservazioni di 1 individuo tra la foce del fiume Ombrone, Macchiozze e la Palude della Trappola, ma senza prove di riproduzione (M. Moretti, P. Brichetti, GA; PG; M. Dragonetti; E. Occhiato in EBN-Italia; M. Colli in GOM-List; ML su Ornitho.it). Raramente osservato anche in mare. 1 ind. il 17/01/2016 alla foce del fiume Ombrone (fonte: COT, archivio PRM).

\section{Cigno selvatico Cygnus cygnus A-1}

Accidentale. 1 ind. intorno al 5 gennaio 1988, dal comportamento schivo, rilevato nella zona della foce del fiume Ombrone (Giovacchini, 1995c). Viene sospesa una seconda osservazione (Giovacchini, 2006).

\section{Oca egiziana Alopochen aegyptiaca $\mathrm{M}$ irr}

In un recente passato risultava esclusa dall'elenco delle specie dell'area protetta (Giovacchini, 2006). 1 ind. il 10/11/1996 e dal 09/02 al 16/03/1997 alle Macchiozze (Giovacchini, 1999). A queste prime si aggiunge 1 ind. il 17/06/2002 nelle Risaie della Trappola (PG). Tra le specie esotiche, presenti in Italia, è tra quelle meno conosciute in termini di impatto sulla biodiversità (Andreotti et al., 2001; Baccetti et al., 2014).

Volpoca Tadorna tadorna M reg, W reg, B irr

In Europa, è specie nidificante in incremento (Staneva \& Burfield, 2017). In Italia, è nidificante e "Vulnerabile" (Peronace et al., 2012). La riproduzione interessa zone umide costiere dove occupa argini, dossi, scanni e barene artificiali, anche in bacini di acqua dolce; in migrazioni e svernamento osservata quasi esclusivamente in saline e lagune (Brichetti \& Fracasso, 2003). A livello locale, rilevata più volte dopo il 2003 la nidificazione in cavità rocciose dell'Argentario, nei pressi di Porto Ercole, secondo un processo di colonizzazione forse ancora in atto (R. Carotti, com. pers.) e, nel 2018, in anfratti rocciosi della più vicina Formica grande di Grosseto (F. Biagini, com. pers.). Nel PRM, occasionale negli anni immediatamente a seguire dall'istituzione dell'area protetta, quasi sempre nel periodo invernale e con singoli individui (Chelini, 1983; C. Castellani, ined.). Successivamente alla conclusione dello scorso secolo lo svernamento diviene regolare per quanto risulti ai più recenti censimenti di gennaio 2013-2017 con cifre comprese tra 8-17 ind. (Arcamone et al., 2007; fonte: COT, archivio PRM). Alcune osservazioni in periodo migratorio: 12 ind. il 03/11/1986 nella Palude della Trappola (PG); più recentemente, 3 ind. il 24/02/2009 alla foce del fiume Ombrone (PG); 4 ind. il 21/02/2016 nella Palude della Trappola (LP in GOM-List). L'accertato primo caso di riproduzione fa riferimento agli stagni retrodunali della Palude della Trappola dove il 16/06/2008 è stata osservata una coppia con 8 pulli (PG); il 25/05/2012, invece, rinvenuto un 1 ind. in mare con 6 pulli nei pressi di Cala di Forno, in presenza di ambienti con cavità rocciose ove la deposizione è avvenuta in condizioni di maggiore sicurezza da rischi di predazione sulle nidiate (Polizia di Stato, Squadra Nautica di Talamone, com. pers.). In quest'ultima zona i primi tentativi di nidificazione risalgono alle stagioni 2009 e 2010 (GA). Nello stesso periodo, individui in corteggiamento o coppie formate anche alla foce del fiume Ombrone con una curiosa segnalazione dalla "Grotta della Fabbrica", nei pressi del canale di Collelungo, dove il 12/04/2011 è stata osservata una coppia in comportamento riproduttivo (I. Tanganelli). Tali atteggiamenti vengono ugualmente rilevati nella Palude della Trappola sino alla stagione 2017 per poi accertare il 23/06/2018 nella Palude della Trappola un nuovo caso di riproduzione con 1 coppia e 5 giovani in acqua (PG). Questi episodi vanno ricondotti al vicino 
nucleo introdotto della Laguna di Orbetello dove la specie ha cominciato a riprodursi dal 1994 (Valle et al., 1995). In seguito inglobata entro un processo di espansione naturale (Baccetti et al., 2014). Nota storica: "1 individuo femmina veniva catturato il 21 febbraio 1907 alla foce dell'Ombrone dal signor Silvio Galigani. È una cattura assai rara per quella regione" (Brogi, 1907).

\section{Casarca Tadorna ferruginea A-1}

Accidentale. Specie precedentemente non rinvenuta nell'area di studio (Giovacchini, 2006). 1 ind. nel PRM alle Macchiozze ed a Vaccareccia dal 29/12/2012 al 16/02/2013, quasi sempre insieme a ind. svernanti di $A n$ ser anser (G. Cappelli; GA; PG; D. Verducci, E. Zarri, A. Quaglierini, M. Taddei, M. Baldini in EBN-Italia; ML su Ornitho.it; Fig. 17). Provenienza dubbia, forse attribuibile ad individui appartenenti a popolazioni recentemente acclimatate in Europa (Brichetti \& Fracasso, 2003; Baccetti et al., 2014).

\section{Marzaiola Spatula querquedula $\mathrm{M}$ reg}

In Europa, è specie nidificante in decremento (Staneva $\&$ Burfield, 2017). Durante il periodo delle migrazioni frequenta zone umide di acqua dolce, interne o costiere; accentuata in primavera secondo una fenologia ad arco (Brichetti \& Fracasso, 2003). Lo svernamento nel bacino del Mediterraneo è un evento irregolare (Zenatello et al., 2014). Nel PRM, compare generalmente sul fini- re del mese di febbraio, trattenendosi quasi sempre sino a metà aprile con gruppi occasionalmente superiori agli 80-90 individui, ad es. circa 100 ind. il 12/03/2007 alla foce del fiume Ombrone (PG). Si segnala la presenza di 1 ind. almeno dal 15/05 al 21/06/1990 nella cava dismessa della Voltina (Giovacchini, 1991). Individuo inanellato: un individuo catturato nella Padule della Trappola il 09/04/1961 era stato inanellato a Tour du Valat, Francia, il 25/04/1959 (Moltoni, 1966).

Mestolone Spatula clypeata M reg, W reg

In Europa, è nidificante stabile (Staneva \& Burfield, 2017). Durante le migrazioni e lo svernamento frequenta vari tipi di zone umide aperte, interne o costiere, anche marine (Brichetti \& Fracasso, 2003). La Macrozona "Maremma Grossetana" è un sito di importanza regionale e nazionale per la specie (Arcamone et al., 2007; Zenatello et al., 2014). Alla creazione dell'area protetta i contingenti presentavano entità oggi difficilmente rilevabili: 480 ind. il 18/01/1979, 700 ind. il 10/01/1981, sempre nella Palude della Trappola (Chelini, 1983; M. Pani ined.). In questo senso, occorre tuttavia precisare che allo stato attuale la sola Riserva Regionale Diaccia Botrona ricopre un'importanza numericamente prioritaria per lo svernamento della specie nell'ambito della stessa Macrozona (Arcamone et al., 2007). Nel PRM, si segnalano tra i più recenti censimenti: 156 ind. il 18/01/2015; 121 ind. il 17/01/2016 (fonte: COT, archivio PRM).

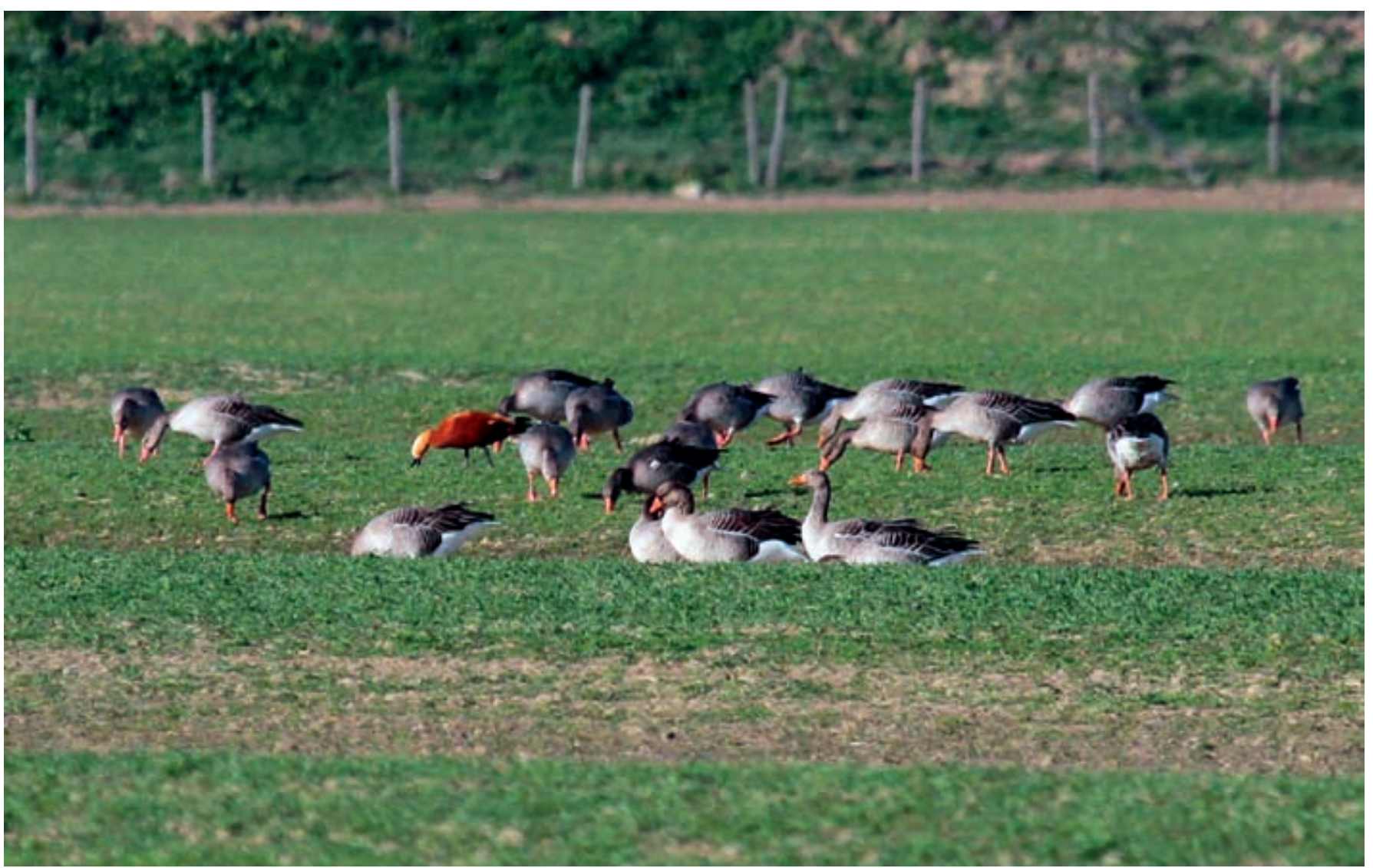

Fig. 17 - Casarca il 29/12/2012 alle Macchiozze. / Ruddy Shelduck on the $29^{\text {th }}$ December 2012 at the Macchiozze (Foto / Photo Giuseppe Anselmi). 


\section{Canapiglia Mareca strepera $\mathrm{M}$ reg, $\mathrm{W}$ reg}

In Europa, è specie nidificante in incremento (Staneva $\&$ Burfield, 2017). Nei periodi delle migrazioni e dello svernamento frequenta più comunemente corpi d'acqua dolce con vegetazione palustre (Brichetti \& Fracasso, 2003). Il PRM ricade con la Macrozona "Maremma Grossetana" in una delle aree più importanti a livello regionale e nazionale per lo svernamento della specie (Arcamone et al., 2007; Zenatello et al., 2014). Con l'istituzione dell'area protetta e immediatamente prima, alcuni conteggi autunno-invernali realizzati nella Palude della Trappola hanno evidenziato negli anni contingenti numericamente modesti, anche se probabilmente sottodimensionati rispetto alla loro reale entità. Si citano ad es. 15 ind. il 18/11/1973 (fonte: Archivio Laboratorio Zoologia Applicata alla Caccia oggi ISPRA); 10 ind. il 25/11/1978 (M. Pani, ined.); 30 ind. il 18/01/1979 (Chelini, 1983). Già nel decennio successivo, grazie anche all'avvio di monitoraggi standardizzati, si rilevano cifre più consistenti, come ad es. 100 ind. il 13/01/1986 nella Palude della Trappola (L. Calchetti, F. Cianchi e C. Giannella in Arcamone \& Tellini, 1987). Ancora più recentemente la popolazione svernante nel PRM sembra attestarsi su cifre simili, per quanto fluttuanti di anno in anno, con punte di 139 ind. il 15/01/2017 (fonte: COT, archivio PRM). Altre presenze di un certo interesse, forse da ricondurre al nucleo gravitante nella vicina Riserva Regionale Diaccia Botrona, come ad es. almeno 300 ind. il 27/12/2001 alla foce del fiume Ombrone (GA e PG). Individui già in migrazione post-riproduttiva: 4 ind. il 05/07/2011 nei laghetti dell'Azienda Agricola di San Mamiliano, Trappola (M. Biondi su Ornitho.it).

\section{Fischione Mareca penelope $\mathrm{M}$ reg, $\mathrm{W}$ reg}

In Europa, è nidificante stabile (Staneva \& Burfield, 2017). Durante le migrazioni e lo svernamento frequenta più facilmente zone umide costiere aperte con ampie distese fangose o sabbiose, saline e paludi salmastre confinanti con pascoli, ma anche fiumi e laghi interni (Brichetti \& Fracasso, 2003). La specie ha subito recentemente una evidente contrazione dei contingenti svernanti nel PRM a vantaggio della vicina Riserva Regionale Diaccia Botrona (Arcamone et al., 2007). Infatti, grazie a questa zona umida, la Macrozona "Maremma Grossetana" nel suo complesso è annoverata tra i siti di importanza internazionale per la specie (Zenatello et al., 2014). Nel periodo 2013-2017, la Palude della Trappola ha ospitato mediamente in inverno circa 150 individui per anno a fronte del precedente periodo 1993-1997 quando si considerava una presenza media di 948 individui per anno (fonte: COT, archivio PRM). Già alla sua costituzione, i contingenti censiti nell'area protetta avevano raggiunto entità tali da permettere di attestare la Palude della Trappola quale sito di rilevanza internazionale per la specie, con 3000 ind. il 18/01/1979 (Chelini, 1984). Ancora sul finire degli anni ' 80 del secolo scorso e nel successivo decennio la sosta diurna in mare di importanti contingenti (per es. circa 250 ind. il 18/12/1988; circa 600 ind. il 15/03/1991), motivata forse anche dall'esercizio venatorio nella vicina Diaccia Botrona, veniva regolarmente registrata nella zona di foce del fiume Ombrone, con osservazioni in mare sino all'abitato di Principina Mare (GA; PG). Alcune altre interessanti presenze: almeno 520 ind. raggruppati alle Macchiozze il 07/01/2001 (PG); 2 ind. il 24/06/2002 sul fosso Razzo, Palude della Trappola (GA); 1 ind. il 03/07/2014 nei chiari della Palude della Trappola (A. Picciau, PG, F. Ferragamo Visconti e G. Visconti). Individuo inanellato: 1 ind. catturato il 10/12/1989 a Principina Mare, era stato inanellato il 02/12/1989 a San Rossore, Pisa (Corsi \& Giovacchini,1995a).

Germano reale Anas platyrhynchos SB, M reg, W reg In Europa, è nidificante stabile (Staneva \& Burfield, 2017). Si riproduce in zone umide costiere o interne di varia origine, naturali o artificiali, anche di ridotte dimensioni, fossati, canali, comunque con vegetazione riparia diversificata e acque preferibilmente lente, risultando più diffuso sino a $500 \mathrm{~m}$ s.1.m.; durante le migrazioni e lo svernamento mostra maggiore plasticità ecologica (Brichetti \& Fracasso, 2003). La Macrozona "Maremma Grossetana" è un sito di importanza regionale per lo svernamento della specie (Arcamone et al., 2007). I numeri che scaturiscono dai censimenti invernali nel PRM potrebbero essere parzialmente condizionati dalla contemporanea presenza di individui appartenenti alle forme domestiche, oltretutto in conseguenza di un loro rilascio clandestino per il problema della potenziale diffusione dell'influenza "aviaria" (Zenatello et al., 2014). L'incidenza numerica di queste forme domestiche rispetto alla forma selvatica è spesso di difficile valutazione. Infatti, resta il fatto più generale che i diffusi ripopolamenti venatori vengono anche effettuati con soggetti fenotipicamente corrispondenti alla forma selvatica (Baccetti et al., 2014). Ad oggi l'area protetta ospita mediamente in inverno, nel periodo 2013-2017, circa 348 individui per anno (fonte: COT, archivio PRM). Nel periodo riproduttivo la specie viene rinvenuta nidificante con singole coppie nell'esteso reticolo idraulico di bonifica, anche secondario, da Talamone a Principina Mare, alle Macchiozze, nella Palude della Trappola e, limitatamente, lungo il fiume Ombrone. Stimabile una presenza minima di 9-10 coppie nidificanti. Prima possibile segnalazione di "anatra germanata": 1 ind. il 17/08/1995 alla cava dismessa della Voltina (PG). Individui inanellati: 1 ind. catturato il 21/12/1983 a Principina Mare, era stato inanellato ad Orbetello, Grosseto, il 03/12/1983 (Bendini \& Spina, 1990); 1 ind. preso in Alberese il 11/01/1989, era stato inanellato il 04/12/1987 ad Orbetello, Grosseto (Corsi \& Giovacchini,1995a).

\section{Codone delle Bahamas Anas bahamensis A-1}

Occasionale nell'area di studio. Specie precedentemente non rinvenuta (Giovacchini, 2006). 1 ind. dal 24 al 27/10/2011 alla foce del fiume Ombrone (ML su Ornitho. it). Specie esotica (Baccetti et al., 2014; Brichetti \& Fracasso, 2015b).

\section{Codone Anas acuta $\mathrm{M}$ reg, W reg}

In Europa, è nidificante, in decremento (Staneva \& Burfield, 2017). Con le migrazioni e lo svernamento frequenta vari tipi di zone umide aperte, interne o costiere, con acque basse e vegetazione sommersa (Brichetti \& Fracasso, 2003). I contingenti in Toscana hanno mostra- 
to una diminuzione molto marcata e, in ogni caso, non determinanti per l'attribuzione alla Macrozona "Maremma Grossetana" della qualifica di sito di importanza regionale e nazionale (Arcamone et al., 2007; Zenatello et al., 2014). Infatti, nel periodo 2013-2017, furono mediamente censiti nell'area protetta 6 individui per anno (fonte: COT, archivio PRM). Interessanti concentrazioni per il PRM attribuibili a migratori: 36 ind. il 05/02/1990 nella Palude della Trappola (PG); 29 ind. il 12/02/2013 alla foce del fiume Ombrone (PG). Occasionalmente estivo: 2 ind. il 05/06/1990 nella Palude della Trappola (PG).

\section{Alzavola Anas crecca $\mathrm{M}$ reg, $\mathrm{W}$ reg}

In Europa, è nidificante con trend sconosciuto (Staneva $\&$ Burfield, 2017). Durante le migrazioni e lo svernamento è rilevata in una grande varietà di ambienti costieri ed interni (Brichetti \& Fracasso, 2003). La Macrozona "Maremma Grossetana" è un sito di importanza regionale e nazionale per lo svernamento della specie (Arcamone et al., 2007; Zenatello et al., 2014). Nel passato, la Palude della Trappola era classificata come area di importanza internazionale con 3500 ind. il 18/01/1979 (Chelini, 1984). Recentemente, nel PRM i contingenti della specie risultano prevalere sulle altre anatre: in questo senso, la media degli individui calcolata nel periodo 2013-2017 indica per l'area protetta la presenza di circa 670 svernanti all'anno (fonte: COT, archivio PRM). Osservazioni estive limitate negli anni, per quanto in apparente crescita negli ultimi 10 circa, se però riferite all'intera Macrozona (PG). Con riferimento alla sola Palude della Trappola sono stati censiti ad esempio: 15 ind. il 4 e 05/06/1990 (PG); 6 ind. il 29/06/2015 (PG).

\section{Fistione turco Netta rufina M irr, W irr}

In Europa, è nidificante con trend incerto (Staneva \& Burfield, 2017). Nelle stagioni di migrazioni ed in quella di svernamento osservata anche in fiumi a corso lento, bacini artificiali, lagune e saline (Brichetti \& Fracasso, 2003). Nel PRM, la specie viene segnalata almeno negli inverni 1989/90, 1991/92 e 2006/07, rispettivamente con 1 coppia, 2 maschi e 1 individuo nelle zone della foce del fiume Ombrone, Macchiozze e Palude della Trappola (Giovacchini \& Anselmi, 1990; Arcamone et al., 2007; CUA in Arcamone \& Puglisi, 2008; GA e PG). Negli anni la permanenza massima registrata fa riferimento ad $1 \mathrm{ind}$. osservato alla foce del fiume Ombrone sino al 20/02/1992 (PG). Reperto museale: 1 ind. abbattuto in Alberese durante l'inverno 1902/03 (Ridolfi, 1908).

\section{Moriglione Aythya ferina $\mathrm{M}$ reg, W irr}

In Europa, è specie nidificante in decremento (Staneva $\&$ Burfield, 2017). Con le migrazioni e lo svernamento preferisce zone umide salmastre o di acqua dolce, costiere o interne, naturali o artificiali, anche di limitata estensione (Brichetti \& Fracasso, 2003). Nei primi anni del PRM la specie viene rilevata con numeri oggi definibili inconsueti, riferiti comunque alla conclusione della stagione invernale: 1500 ind. il 08/02/1976 nella Palude della Trappola (Chelini, 1983). Già sul finire degli anni '80 del secolo scorso, ed immediatamente dopo, si rafforza il quadro fe- nologico della specie con lo svernamento irregolare, ma con gruppi più ridotti, generalmente 10-50 individui (Arcamone et al., 2007). Recentemente si segnalano: 13 ind. il 21/01/2013 nella Palude della Trappola (GA); 16 ind. il 06/01/2017 nei chiari interni della Trappola (M. Carabella, F. Pianezza, PG su Ornitho.it). Vanno messi in evidenza i contingenti gravitanti nella limitrofa cava dismessa della Voltina dove, apparentemente sino all'inizio di questo secolo, vengono osservati più volte movimenti di individui in rimessa diurna provenienti anche dal PRM (GA; PG; F. Corsi e C. Castellani). In tal senso, comprensivo anche del periodo riproduttivo, per la Voltina si citano ad es. 1 coppia il 07/07/1996 (PG); 126 ind. il 10/01/2001 (PG e GA).

\section{Moretta tabaccata Aythya nyroca $\mathrm{M}$ irr, W irr}

In Europa, è nidificante con trend sconosciuto (Staneva $\&$ Burfield, 2017). Durante le migrazioni e lo svernamento preferisce zone umide acqua dolce poco profonde, costiere o interne, naturali o artificiali, anche di limitata estensione purchè con fasce di vegetazione palustre emergente (Brichetti \& Fracasso, 2003). Specie la cui comparsa nel PRM è occasionale, generalmente con singoli individui. Sono segnalate le presenze invernali di 1 ind. il 09/12/1989 ed ancora di 1 ind. maschio il 29/12/1993, unicamente alla foce del fiume Ombrone (P. Politi, L. Becherucci e N. Baccetti in Arcamone \& Tellini, 19911992). Effettivi casi di svernamento vengono irregolarmente osservati da oltre venticinque anni nella limitrofa cava dismessa della Voltina (Giovacchini, 1991; Arcamone, 1997a). Nel successivo 1994 la specie viene più volte rinvenuta in migrazione, ad es. 1 coppia il 24/03/1994 nella Palude della Trappola (PG); 1 ind. il 17/09/1994 presso l'invaso artificiale del Collecchio (M. Marinelli). Più recentemente: 1 ind. il 07/04/2000 (P. Arrigucci e D. Germani); 1 ind. il 21/12/2015, in entrambi i casi alla foce del fiume Ombrone (E. Occhiato in EBN-Italia). Negli ultimi anni rinvenuta in inverno anche nella Palude della Trappola: 1 ind. il 24/12/2018 (PG).

\section{Moretta Aythya fuligula M irr, W irr}

In Europa, è nidificante stabile (Staneva \& Burfield, 2017). Con le migrazioni e lo svernamento preferisce zone umide acqua dolce mediamente profonde, costiere o interne, naturali o artificiali (Brichetti \& Fracasso, 2003). Nel PRM, è specie migratrice irregolare, svernante irregolare. Compare sempre con un numero molto limitato di esemplari, generalmente non superiore ai 4-5 ind. con l'unica eccezione di 14 ind. il 16/01/1989 alla foce del fiume Ombrone (Arcamone et al., 2007). Le condizioni climatiche rilevabili nelle regioni d'oltralpe possono comunque influire pesantemente sul numero di individui rintracciabili nel Paese in inverno (Casini \& Genero, 1992; Brichetti \& Fracasso, 2003). Si segnala: 1 ind. il 13/01/1986 nella Palude della Trappola (GA, E. Arcamone e F. Cianchi in Arcamone \& Tellini, 1987); sino a 2 maschi osservati dal 02/02 al 09/03/1993 nella Palude della Trappola (PG); 4 ind. il 15/01/2017 nella Palude della Trappola (fonte: COT, archivio PRM); 1 femmina il 28/12/2017 nel Chiaro Grande della Trappola (C. Martelli e PG in GOM-List). 
Moretta grigia Aythya marila $\mathrm{M}$ irr, $\mathrm{W}$ irr

In Europa, è specie nidificante in decremento (Staneva \& Burfield, 2017). In inverno frequenta acque marine poco profonde, lagune e foci fluviali (Brichetti \& Fracasso, 2003). Raro visitatore nel PRM, con effettivi casi di svernamento di singoli individui alla foce del fiume Ombrone nelle sole stagioni invernali 1989/90 e 1998/99 (Arcamone et al., 2007). In occasione dell'ultimo inverno summenzionato, la specie è stata rinvenuta, sempre nella medesima località, almeno dal 01/01/1999 (GA). Precedentemente si segnala: 1 ind. dal 05 al 19/02/1989 alla foce del fiume Ombrone (E. Arcamone, N. Baccetti e F. Cianchi in Arcamone \& Tellini, 1991-1992).

\section{Edredone Somateria mollissima M irr, W irr}

In Europa, è specie nidificante in decremento (Staneva $\&$ Burfield, 2017). Durante le migrazioni e lo svernamento rilevato in acque marine, lagune, golfi, alle foci dei fiumi con fondali di profondità medio-bassa (Brichetti \& Fracasso, 2003). Nel PRM, lo svernamento della specie viene rilevato nella stagione 1988/89 (Arcamone et al., 2007). I movimenti invasivi allora registrati lungo le coste toscane inducono a descrivere una situazione numericamente eccezionale tale da interessare anche l'area protetta (Cavallini, 1992). Successivamente, si evidenzia, negli anni 2005 e 2006, una maggiore frequentazione del PRM, specialmente alla foce del fiume Ombrone, per lo più con individui giovani presenti anche in estate: 2 ind. dal 09/07 al 18/08/2006 (M. Dinetti; M. Cadanelli in EBN-Italia). Occasionali osservazioni invernali negli anni: 1-3 ind. almeno dal 27/11 al 8/12/2005 alla foce del fiume Ombrone (E. Bosi in EBN-Italia; GA; F. Farsi; L. Pardini, E. Occhiato in EBN-Italia); 1 ind. il 12/12/2010 alla foce del fiume Ombrone (PG) (Fig. 18). I dati raccolti in periodo migratorio si riferiscono specialmente a movimenti autunnali negli anni 1988, 1990, 1999 e 2006 (P. Cavallini; P. Arrigucci e L. Buratta; R. Garavaglia; M. Lausetti; M. Mei; PG).

Orco marino Melanitta fusca M irr ?, W irr

In Europa, è specie nidificante in decremento (Staneva \& Burfield, 2017). Con le migrazioni e lo svernamento frequenta acque marine poco profonde, anche a notevole distanza dalla terraferma (Brichetti \& Fracasso, 2003). La specie ha svernato lungo la costa del PRM nella sola stagione 1988/89, per quanto sia l'unica anatra marina presente regolarmente in Toscana in questo periodo dell'anno quando più spesso è rilevata nei soli settori settentrionali (Arcamone et al., 2007). Le scarne segnalazioni comprendono 4 ind. il 12/12/1988 alla foce del fiume Ombrone (I. Boschi in Arcamone \& Tellini, 1991-1992); 9 ind. in volo il 19/12/1999 alla foce del fiume Ombrone (D. Occhiato, com. pers.); 3 ind. maschi il 17/11/2001 alle Cannelle di Talamone (GA).

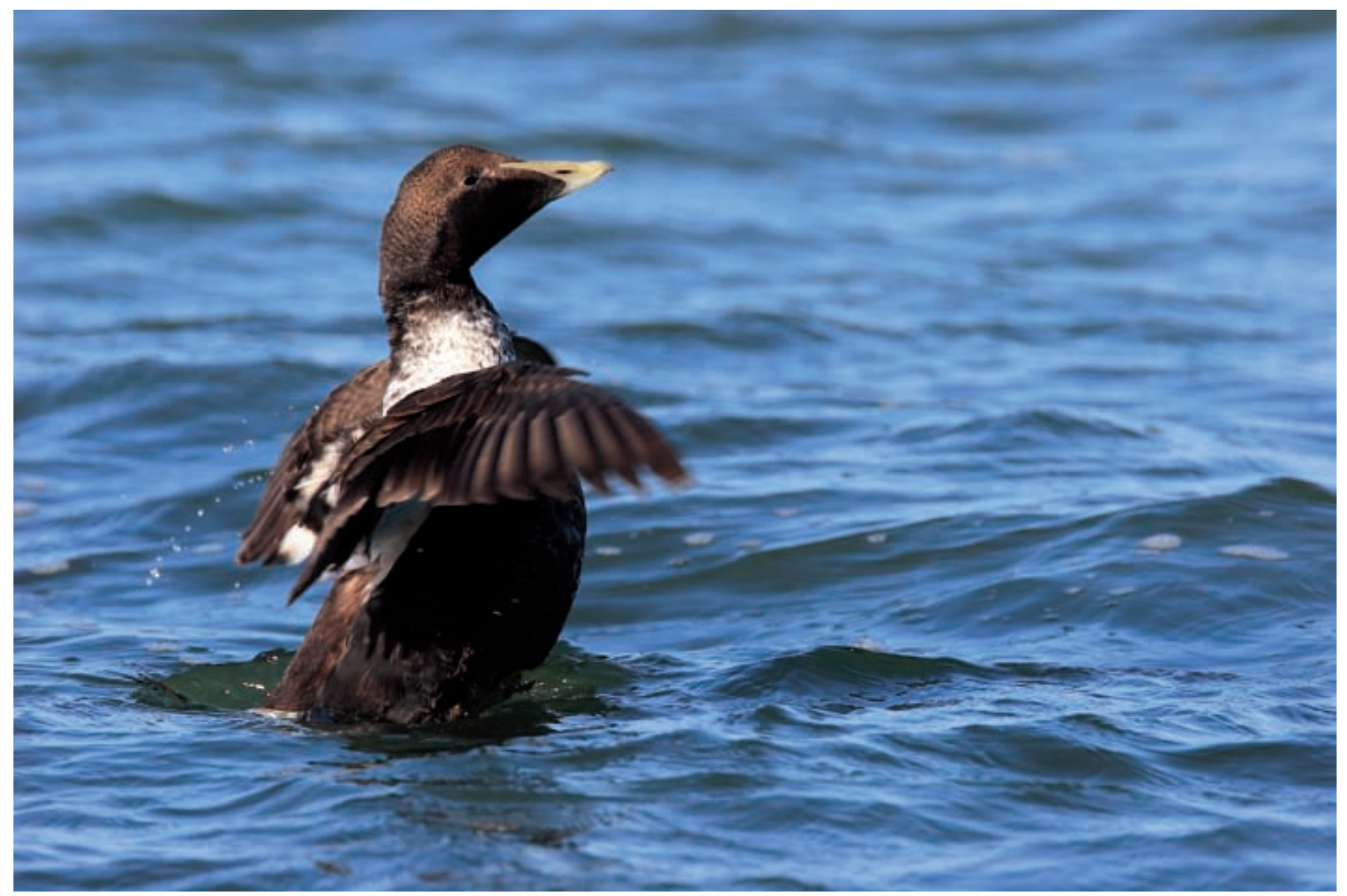

Fig. 18 - Edredone. / Common Eider (Foto / Photo Fabio Cianchi). 
Orchetto marino Melanitta nigra $\mathrm{M}$ irr, W irr

In Europa, è nidificante con trend sconosciuto (Staneva \& Burfield, 2017). In migrazioni e svernamento frequenta acque marine relativamente profonde, anche a notevole distanza dalla costa (Brichetti \& Fracasso, 2003). Raro visitatore nel PRM con un maggior numero di segnalazioni che giungono come svernante dalla costa settentrionale della Toscana, risultando spesso in associazione con altre anatre marine (Arcamone et al., 2007). Per l'area protetta si registra nel primo decennio di questo secolo: 1 ind. il 17/02/2001 alla foce del fiume Ombrone (F. Bodrero in EBN-Italia); 1 femmina svernante dal 17/11/2001 al 22/02/2002 alla foce del fiume Ombrone e lungo un suo tratto più interno ed ancora, lungo la costa sino a Principina Mare (F. Pezzo; GA, G. Manganelli e G. Cappelli; PG; S. Celletti e R. Papi); 2 ind. il 28/12/2002 alla foce del fiume Ombrone (L. Vanni in Arcamone \& Puglisi, 2006). Nel successivo decennio: 2 ind. il 25/02/2011 alla foce del fiume Ombrone (M. Cappelli su Ornitho.it); 2 ind. il 19/01/2014 alla foce del fiume Ombrone (fonte: COT, archivio PRM).

Quattrocchi Bucephala clangula $\mathrm{M}$ irr, W irr

In Europa, è nidificante stabile (Staneva \& Burfield, 2017). Durante le migrazioni e lo svernamento frequenta più facilmente acque marine costiere, poco profonde, foci fluviali, lagune e valli salmastre (Brichetti \& Fracasso, 2003). Nel PRM, compare irregolarmente e con singoli individui, più facilmente nelle stagioni invernali. In questo periodo dell'anno si evidenzia: 1 ind. dal 16/12/1988 al 19/02/1989 alla foce del fiume Ombrone (GA; GA, N. Baccetti e F. Cianchi in Arcamone \& Tellini, 1991-1992);
1 ind. femmina dal 22/12/2001 al 04/01/2002 alla foce del fiume Ombrone (GA; S. Celletti e R. Papi); 1 ind. femmina dal 14/12/2014 al 25/01/2015 al Chiaro del Porciatti, Palude della Trappola (PG e M. Colli; GA; Fig. 19). In migrazione: 1 ind. maschio dal 13/02/1999 al 18/02/1999 alla foce del fiume Ombrone (E. Occhiato in Arcamone \& Puglisi, 2006; PG).

\section{Pesciaiola Mergellus albellus A-1}

Accidentale. 2 maschi il 28/11/2004 sul mare tra le Cannelle ed il Frontone di Talamone (GA).

\section{Smergo maggiore Mergus merganser M irr, W irr}

In Europa, è specie nidificante stabile (Staneva \& Burfield, 2017). Con le migrazioni e lo svernamento frequenta più facilmente acque marine costiere, poco profonde, foci fluviali e acque dolci dell'interno (Brichetti \& Fracasso, 2003). Migratore a corto raggio, con esigue presenze invernali al di fuori dell'Italia settentrionale (Santolini \& Pandolfi, 1992; Zenatello et al., 2014). Nel PRM, i casi di svernamento accertati risalgono agli inverni 1988/89 e 2005/06 lungo costa (Giovacchini, 1995d; Arcamone et al., 2007). Per l'ultima stagione invernale menzionata, le osservazioni, riferite ad 1 ind. femmina, sono proseguite sino al 20/01/2006 nei pressi di Marina di Alberese (GA; G. Prola in EBN-Italia). Interessanti, a queste latitudini, le ripetute segnalazioni primaverili di individui in migrazione che, per questa specie, ha una portata sconosciuta: 2 ind. il 11/04/1996 a Marina di Alberese (GA e S. Celletti); 2 maschi e 1 femmina il 18/04/2004 a Marina di Alberese (GA); 2 ind. il 31/03/2012 nei pressi della foce del fiume Ombrone (C. Martelli in GOM-List).

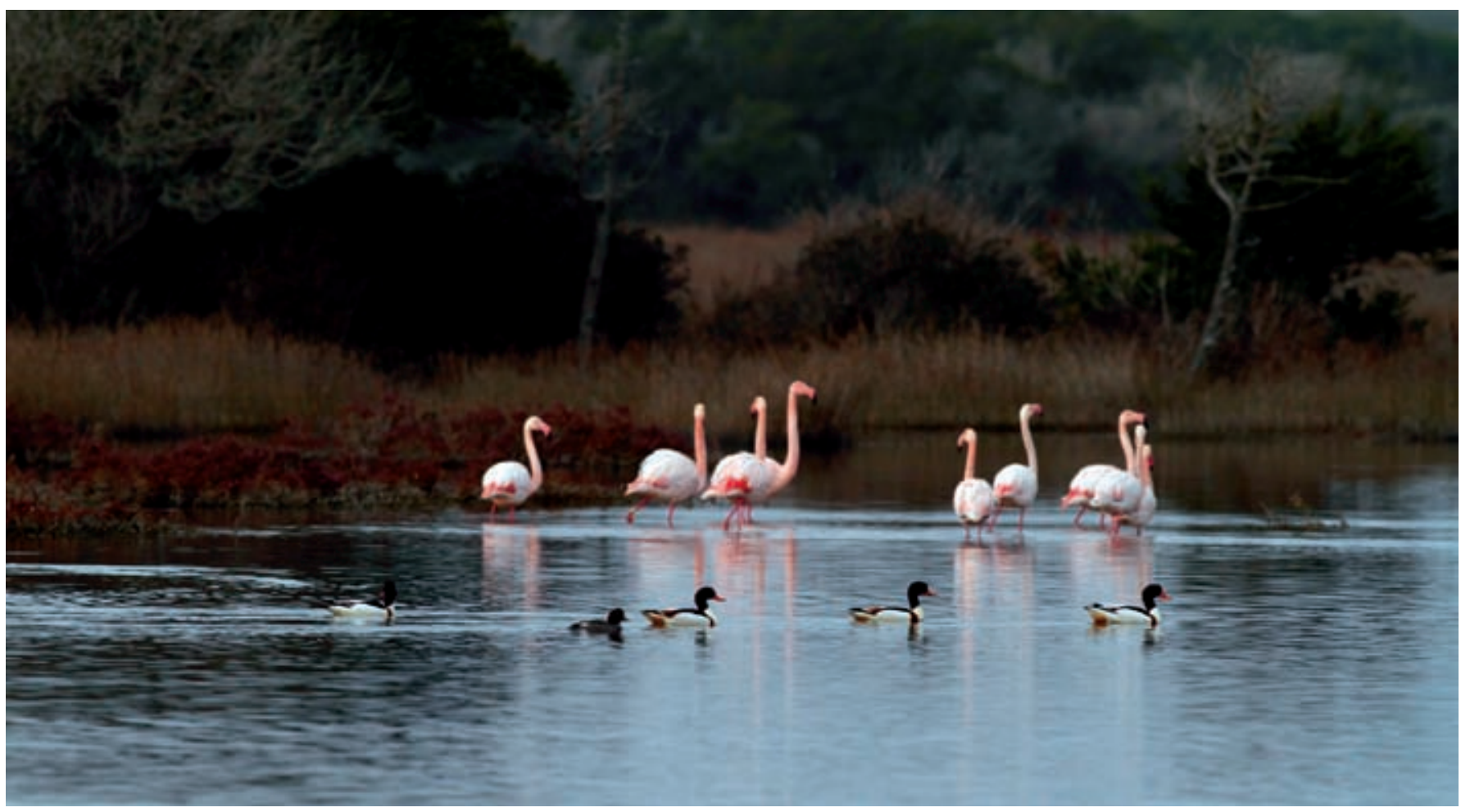

Fig. 19 - Quattrocchi, volpoche e fenicotteri il 22/01/2015 nel Chiaro del Porciatti, Palude della Trappola. / Goldeneye, shelducks and flamingos on the $22^{\text {nd }}$ January 2015 at the Porciatti's ponds and Trappola's marsh (Foto / Photo Giuseppe Anselmi). 
Smergo minore Mergus serrator $\mathrm{M}$ reg, W reg

In Europa, è specie nidificante in decremento (Staneva $\&$ Burfield, 2017). Durante le migrazioni e lo svernamento frequenta più facilmente acque marine costiere spesso rocciose, poco profonde, foci fluviali, lagune e valli salmastre (Brichetti \& Fracasso, 2003). Nel PRM, il primo indizio di svernamento porta alla recente stagione 2010/11 quando, durante i censimenti, è stato osservato 1 ind. il 16/01/2011 nei pressi della foce del fiume Ombrone (fonte: COT, archivio PRM). Se comparate con i dati disponibili alla fine del secolo scorso, le segnalazioni di presenze invernali finora raccolte permettono oggi di descrivere un quadro complessivo sensibilmente diverso rispetto al passato (Arcamone \& Tellini, 1987). Infatti, lo svernamento sembrerebbe assumere un carattere di regolarità dall'inverno 2014/15 comprendendo anche il dicembre 2017 (GA; PG e SL; M. Dragonetti in GOM-List, LP in GOM-List; M. Apuleo e A. Montemaggiori su Ornitho. it). Queste le massime concentrazioni osservate: 3 ind. il 18/12/2014 alla foce del fiume Ombrone (PG e GA); 4 ind. il 02/12/2016 alla foce del fiume Ombrone (A. Fabiani su Ornitho.it). Piccoli nuclei composti al massimo da 4-5 individui, per lo più femmine, sono stati rinvenuti con maggiore frequenza nel periodo della migrazione autunnale lungo l'intera costa dell'area protetta dagli anni 19931995 (Principina Mare, Collelungo, Le Cannelle, Talamone). Occasionale nella Palude della Trappola: 1 ind. femmina il 9 e 10/11/1995 (GA e PG); dal 6 al 10/04/1997 (M. Porciani e GA; PG). Massima concentrazione registrata: 8 ind. il 14/11/2001 in volo lungo il fiume Ombrone (GA).

\section{GALLIFORMES}

\section{Phasianidae}

\section{Starna Perdix perdix A-1}

Segnalazione riferita ad un rilascio, a nord del PRM, di esemplari nella vicina Zona di Ripopolamento e Cattura "San Lorenzo" per la stagione venatoria 1991/92, con individui rinvenuti anche nell'area protetta il 17/09/1991, nei pressi di Principina Mare (PG). Come atteso, l'immissione non risulta aver dato origine ad alcuna popolazione vitale. Intervento operato all'interno dell'areale riproduttivo storico (Baccetti \& Meschini, 1986).

\section{Quaglia Coturnix coturnix $\mathrm{M}$ reg, B}

In Europa, è nidificante con trend fluttuante (Staneva \& Burfield, 2017). Si riproduce in ambienti aperti erbosi diversificati con cespugli e alberi molto sparsi, generalmente sino a 1800 m s.l.m. (Brichetti \& Fracasso, 2004). Specie migratrice regolare, nel PRM con casi di nidificazione nelle zone di Alberese e Collecchio almeno negli anni 1991 e 2000 (I. Nerozzi, com. pers; PG). Individui in entrata dal mare venivano ancora rilevati piuttosto frequentemente alla fine degli anni '80 e nei primi anni del decennio successivo del secolo scorso, in particolare nelle prime ore del mattino. Osservazione più precoce: 2 ind. il 01/03/1988 sulla spiaggia di Principina Mare (PG). Nell'area protetta, durante il periodo riproduttivo, frequenta tutti i settori interni di pianura purchè aperti, con preferenze verso i prati stabili e le coltivazioni di frumento, in subordine gli incolti. Stimabile una presenza minima di 2-4 coppie nidificanti.

\section{Fagiano comune Phasianus colchicus SB}

Specie negli anni oggetto di immissioni a scopo venatorio in aree esterne al PRM. Risulta ben rappresentata e vitale nei settori interni di pianura, specialmente nelle aree agricole con boschetti e/o filari di vegetazione arborea ed ancora lungo i margini della golena del fiume Ombrone come del reticolo idraulico secondario ed ancora, delle pinete litoranee. Si rinviene meno facilmente in prossimità delle zone umide più estese dell'area protetta. Popolazioni di incerta attribuzione tassonomica (Baccetti et al., 2014). Stimabile una presenza minima di 30-40 coppie nidificanti.

\section{Fagiano argentato Lophura nycthemera A-1}

Occasionale nell' area di studio. Specie precedentemente non rinvenuta (Giovacchini, 2006). 1 ind. il 14/01/2013 nei pressi della foce del fiume Ombrone, insieme ad una decina di Phasianus colchicus (LP in GOM-List). Sfuggito sicuramente alla cattività (Andreotti et al., 2001; Baccetti et al., 2014).

\section{Fagiano dorato Chrysolophus pictus A-1}

Occasionale nell' area di studio. Specie precedentemente non rinvenuta (Giovacchini, 2006). 2 ind. il 09/09/2012 a Cala di Forno (GA e G. F. Martini). Sfuggito sicuramente alla cattività (Baccetti et al., 2014).

\section{GAVIIFORMES}

\section{Gaviidae}

\section{Strolaga minore Gavia stellata M irr? W irr}

In Europa, è nidificante con trend sconosciuto (Staneva $\&$ Burfield, 2017). Durante le migrazioni e lo svernamento utilizza tratti di costa marini, sopratutto alle foci dei fiumi, ma anche lagune e laghi costieri (Brichetti \& Fracasso, 2003). Specie di difficile rilevabilità, con individui osservabili anche oltre un miglio nautico dalla riva. Più in generale, si disperde su ampi tratti di costa marina ove la disponibilità di cibo, le situazioni meteorologiche e la tranquillità dei siti ne condizionano l'occupazione. Nel PRM, questa indicazione trova conferma con singole presenze o piccoli gruppi rinvenibili tra Principina Mare e Cala di Forno, tra l'altro associandosi a volte con la congenere Gavia arctica, durante l'intero periodo di frequentazione che, nella norma, è limitato all'intervallo dicembre-febbraio (Arcamone et al., 2007). In questo periodo dell'anno, tra le più recenti conferme, si citano quelle delle stagioni 2005/06, 2006/07 e 2012/13 (GA; PG; G. Chiancianesi e C. Marti in EBN-Italia). I gruppi generalmente non superano i 3 individui. Massime concentrazioni, tra le più recenti osservazioni: 5 ind. il 04/01/2002 alla foce del fiume Ombrone (S. Celletti e R. Papi); 6 ind. il 06/01/2013 sul mare a Marina di Alberese (E. Zarri in EBN-Italia). Presenze sporadiche tra maggioluglio: 3 ind. il 04/05/1997, in abito estivo, in vicinanza della foce del fiume Ombrone (GA e M. Porciani). 
Strolaga mezzana Gavia arctica $\mathrm{M}$ reg, W irr

In Europa, è specie nidificante in decremento (Staneva $\&$ Burfield, 2017). Durante le migrazioni e lo svernamento utilizza tratti di costa marini, sopratutto alle foci dei fiumi, anse portuali, ma anche laghi costieri e lagune (Brichetti \& Fracasso, 2003). Come la congere Gavia stellata è specie di difficile rilevabilità dal momento che individui vengono osservati anche ad oltre un miglio nautico dalla riva. Frequenta ampi tratti di costa marina con numeri che, nella generalità degli anni, risultano a livello regionale più abbondanti rispetto a quelli della precedente specie (Arcamone et al., 2007). Disponibilità di cibo in abbondanza, situazioni meteorologiche favorevoli e assenza di disturbo ne condizionano la presenza. Nel PRM, verificandosi queste condizioni, si segnalano singole presenze o gruppi composti mediamente da 2-3 individui osservabili più facilmente tra Principina Mare e Cala di Forno, raramente nelle zone interne della Palude della Trappola. Rilevata, a volte, con Gavia stellata. Osservazioni normalmente circoscritte all'intervallo novembre-febbraio (Giovacchini, 1995 e). Tra le più recenti conferme di presenza si riportano le stagioni 2011/12, 2012/13 e 2016/17 (PG; GA e L. Minucci; G. Chiancianesi e C. Marti in EBN-Italia) (Fig. 20). Massime concentrazioni, tra le più recenti osservazioni: 4 ind. il 27/11/2011 alla foce del fiume Ombrone (PG); 3 ind. il 27/01/2013 a Marina di Alberese (G. Chiancianesi e C. Marti in EBN-Italia); 3 ind. il 17/01/2017 a
Marina di Alberese (PG). Occasionale nella Palude della Trappola: 1 ind. il 03/01/2001 (GA). Le presenze diventano sporadiche tra giugno-settembre. A questo proposito si riporta: 1 ind. il 18/08/1998 a Collelungo (GA). Nota storica: "Colymbus glacialis giovane. Ucciso in Bocca d'Ombrone nella Tenuta Ponticelli a Grosseto 16 gennaio 1909 " Formichi (legit ?) [erroneamente identificato come una Strolaga maggiore, in realtà si tratta di una Strolaga mezzana giovane. Esemplare tassidermizzato conservato presso il Centro Visite "La Sterpaia" - Parco Regionale di Migliarino, San Rossore, Massaciuccoli].

\section{PROCELLARIIFORMES}

\section{Procellariidae}

Berta maggiore Calonectris diomedea $\mathrm{M}$ reg, $\mathrm{W}$ irr?

La specie è segnalata in diminuzione in Europa come nidificante (Staneva \& Burfield, 2017). Dal comportamento pelagico, e quindi di difficile rilevabilità dalla riva, è nidificante in poche località insulari toscane, con osservazioni imputabili a movimenti dispersivi o a individui in migrazione (Brichetti \& Fracasso, 2003). Fino alla conclusione dello scorso secolo si poteva evidenziarne l'assenza in inverno lungo la costa del PRM, perlomeno alla distanza di un miglio nautico dalla riva (Baccetti \&

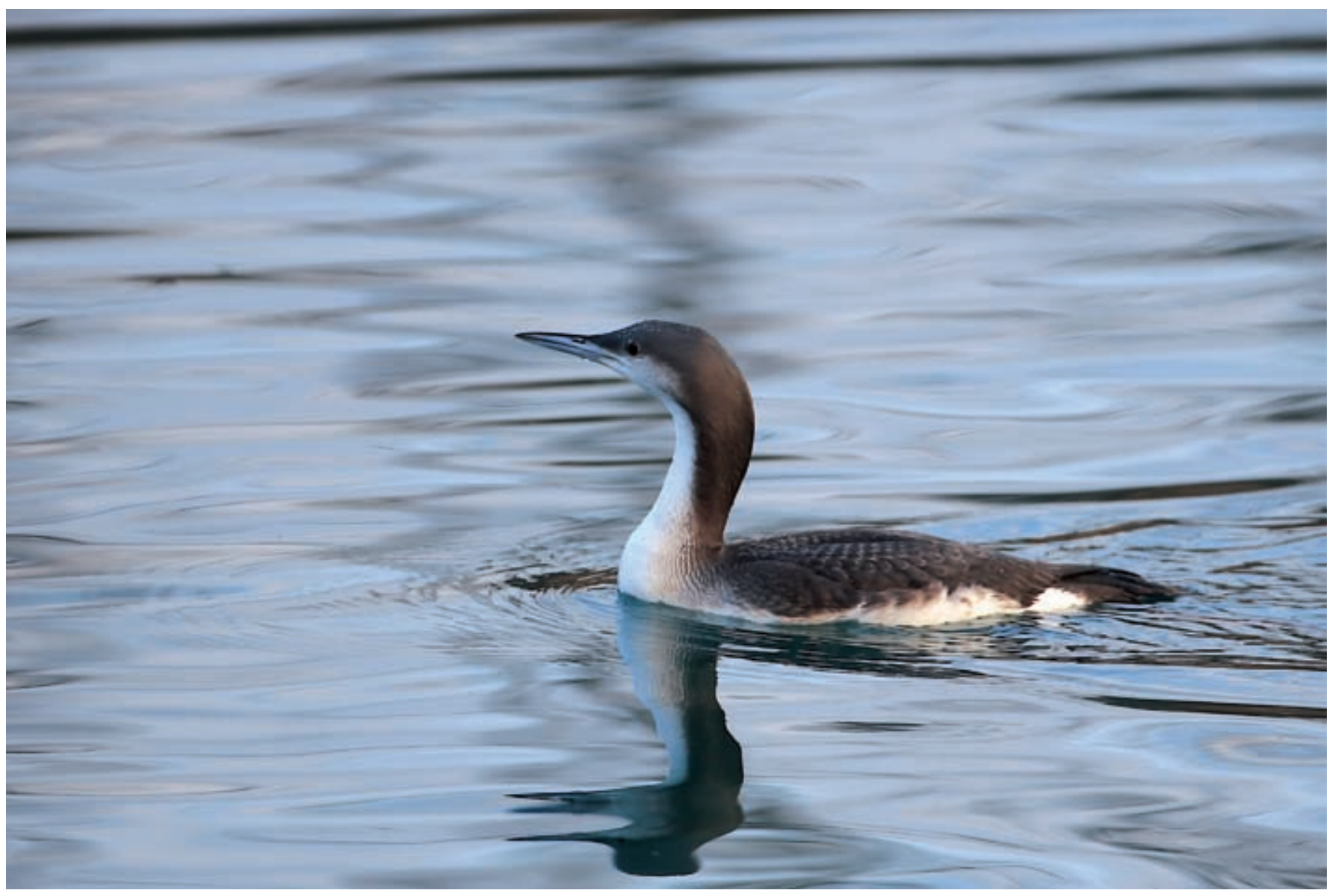

Fig. 20 - Strolaga mezzana. / Arctic Loon (Foto / Photo Fabio Cianchi). 
Roselli, 1997). Infatti, nella stagione 1996/97 ed in quella 1999/2000, così come negli anni successivi, si assiste ad un graduale aumento, per quanto irregolare, di osservazioni invernali (PG e GA; F. Corsi; M. Lausetti; S. Celletti e R. Papi). Tra le massime concentrazioni invernali in questo secolo si segnalano: 4 ind. il 23/12/2009 a Principina Mare (PG). Stormi più numerosi sono presenti nel periodo primaverile, tra marzo e aprile, con gruppi composti da 60-200 individui ed oltre, notati specialmente a Marina di Alberese negli anni 2009 e 2012 (F. Corsi e M. Dragonetti; GA; M. Passarella su Ornitho.it).

\section{Berta minore Puffinus yelkouan $\mathrm{M}$ reg, W reg}

In Europa, è nidificante con trend incerto (Staneva \& Burfield, 2017). Dal comportamento pelagico, e quindi di difficile rilevabilità dalla riva, è nidificante anche in alcune isole toscane, con le osservazioni da imputare più facilmente a lunghi movimenti per il foraggiamento o ad individui in migrazione (Brichetti \& Fracasso, 2003). Anche nel PRM le presenze invernali, non essendo strettamente vincolate alle zone riproduttive, sono più facilmente riconducibili agli spostamenti dei banchi di pesce azzurro, con interessanti concentrazioni rilevate: 2000-3000 ind. il 25/01/1991 alla foce del fiume Ombrone (Anselmi,1995a; S. Benucci e D. Biagini in Arcamone \& Barbagli, 1995-1996); almeno 600 ind. il 07/01/2002 a Marina di Alberese (PG). In migrazione rilevate anche presenze mediamente costanti su brevi periodi: $350-450$ ind. il 17/05/2009 a Marina di Alberese (F. Corsi e M. Dragonetti), oppure di rilievo per la concentrazione: 3000 ind. il 29/03/1996 sempre a Marina di Alberese (GA e S. Celletti).

\section{PODICIPEDIFORMES}

\section{Podicipedidae}

Tuffetto Tachybaptus ruficollis $\mathrm{M}$ reg, $\mathrm{W}$ reg, B

In Europa, è nidificante con trend incerto (Staneva \& Burfield, 2017). Nidifica in zone umide di acqua dolce ferma o corrente, naturali o artificiali, poco profonde, anche di ridotte dimensioni con maggiori presenze sino a $500 \mathrm{~m}$ s.l.m.; nei periodi delle migrazioni ed in inverno è rilevato, per esempio, in acque dolci o debolmente salmastre, in laghetti urbani ed in canali di bonifica (Brichetti $\&$ Fracasso, 2003). Nel PRM, è specie presente durante tutto l'anno. Ad oggi la Palude della Trappola e la foce del fiume Ombrone hanno ospitato mediamente in inverno, nell'intervallo 2013-2017, 11 individui per anno (fonte: COT, archivio PRM). In questo periodo, così come facilmente durante le migrazioni, le osservazioni possono avvenire anche in alcuni tratti più interni dell'area protetta dove esistono piccoli bacini artificiali per l'irrigazione (nella zona del Collecchio): 8 ind. il 31/12/2014 nel laghetto contiguo al Podere Francesco Vivarelli Colonna (PG). Occasionale sul mare, a causa anche delle condizioni meteorologiche spesso avverse: 1 ind. il 31/12/1996 a Principina Mare con i chiari interni gelati (PG). Nel periodo migratorio, interessanti concentrazioni nel Canale di Bonifica di Talamone: sino a 41 ind. il 07/09/2010 lungo circa $2,5 \mathrm{~km}$ (GA). La riproduzione ha luogo in zone umide d'acqua dolce, di diversa estensione e poco profonde, risultando comunque circoscritta a poche aree quali il canale Essiccatore e gli stagni delle Macchiozze, entrambi in Alberese nonchè lungo il Canale di Bonifica di Talamone, ad es. 1 coppia il 18/05/2001 alle Macchiozze; 1 coppia il 12/05/2014 nei pressi delle Macchiozze (PG; GA; A. Gianni; M. Mei; L. Buratta; M. Pacchiarotti). A volte la nidificazione avviene al limite del calendario riproduttivo: 1 ind. in cova il 25/08/2012 nel Canale di Bonifica di Talamone (GA; Fig. 21). Stimabile una presenza minima di 1-3 coppie nidificanti.

\section{Svasso collorosso Podiceps grisegena M irr, W irr}

In Europa, è specie nidificante in incremento (Staneva $\&$ Burfield, 2017). In Italia, per quanto sia un migratore regolare, le presenze invernali sono più facilmente condizionate dalle situazioni climatiche e ambientali, con il Paese posto al margine dell'areale di svernamento, frequentando in particolare acque marine portuali, riparate, lagune ed estuari (Brichetti \& Fracasso, 2003). Nel PRM, è specie rilevata quasi esclusivamente alla foce del fiu-

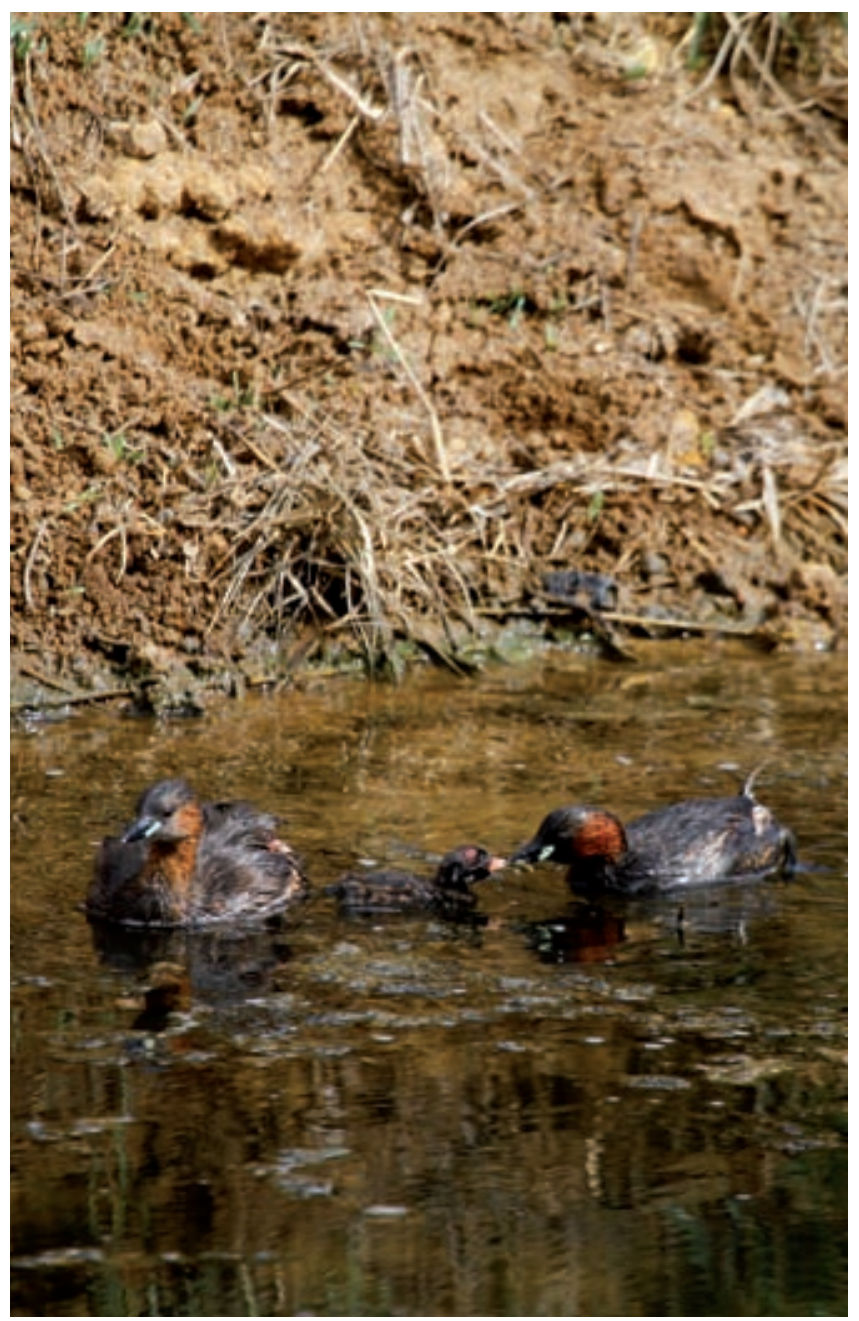

Fig. 21 - Tuffetti con giovane il 25/08/2012 nel Canale di Bonifica di Talamone. / Little Grebes and young on the $25^{\text {th }}$ August 2012 in the Talamone drainage channel (Foto / Photo Giuseppe Anselmi). 
me Ombrone, sempre con singoli individui. Nell'area protetta e nell'immediato circondario, i dati decorrono dall'inverno 1992/93 quando 1 ind. è stato osservato il 13/01/1993 nel golfo di Talamone (PG). Successivamente se ne rileva la presenza con 1 ind. il 30/12/1993 alla foce del fiume Ombrone (N. Baccetti in Arcamone \& Puglisi, 2006). L'unico effettivo caso di svernamento conosciuto fa riferimento ad 1 ind. osservato nella stessa zona del precedente, dal 25/11/2001 al 14/04/2002 (PG; A. Canci; F. Pezzo; S. Celletti e R. Papi).

\section{Svasso maggiore Podiceps cristatus $\mathrm{M}$ reg, W reg}

In Europa, è nidificante in decremento (Staneva \& Burfield, 2017). Fuori dalla stagione riproduttiva preferisce laghi d'acqua dolce naturali con fondali profondi e ricchi di pesci; localmente anche acque marine, zone umide salmastre costiere e bacini artificiali antropizzati (Brichetti \& Fracasso, 2003). Durante le migrazioni ed in inverno presenze regolari nel PRM, quasi sempre isolate o con un massimo di 2-3 individui, per un totale di circa 10 individui per stagione, distribuiti lungo il fiume Ombrone sino alla sua foce, lungo la costa marina e, limitatamente, nella Palude della Trappola. L'area protetta non rappresenta un sito di rilevanza per la specie in inverno, verosimilmente in considerazione della disponibilità di ampi specchi lacustri ricchi di pesci posti più a sud ove si rinvengono concentrazioni numeriche più significative (Arcamone et al., 2007; Monti, 2012a). Le zone più riparate e forse con una maggiore concentrazione di cibo costituiscono in qualche modo un'attrazione per la specie, per quanto modesta: 7 ind. insieme il 21/01/2000 ed il 04/01/2002 nel golfo di Talamone (PG).

\section{Svasso cornuto Podiceps auritus M irr}

In Europa, è nidificante in decremento (Staneva \& Burfield, 2017). Oltre il periodo riproduttivo gli individui appartenenti alle popolazioni orientali assumono un comportamento erratico, spostandosi anche verso le regioni settentrionali e centrali del Paese ove frequentano, in particolar modo, le acque marine costiere, foci fluviali, laghi d'acqua dolce dell'interno e laghi salmastri (Brichetti \& Fracasso, 2003). Nel PRM, è specie migratrice irregolare con almeno due segnalazioni tra le più recenti: 1 ind. il 01/10/2002 alla foce del fiume Ombrone (PG e L. Vanni); 2 ind. il 11/02/2004 nella Palude della Trappola (PG).

\section{Svasso piccolo Podiceps nigricollis $\mathrm{M}$ reg ?, W irr}

In Europa, è nidificante con trend sconosciuto (Staneva \& Burfield, 2017). Durante le migrazioni e lo svernamento frequenta acque marine, costiere e corpi idrici più interni (Brichetti \& Fracasso, 2003). Nel PRM, è più facilmente incontrato con isolate presenze o a gruppi di 2 individui alla foce del fiume Ombrone e nella Palude della Trappola, raramente sul mare o in limitati contesti interni d'acqua dolce. La specie, a queste latitudini, mostra normalmente un comportamento erratico durante il periodo invernale che potrebbe giustificarne l'assenza per una parte di quella stagione e l'irregolarità dello svernamento nell'area protetta (Arcamone et al., 2007). Segnalazioni invernali più recenti: 1 ind. il 26/12/2002 alle Macchioz- ze (PG); 1 ind. il 10/01/2009 nella Palude della Trappola (PG); 1 ind. il 03/01/2012 alla foce del fiume Ombrone (PG); 2 ind. almeno dal 24 al 31/12/2018 nella Palude della Trappola (PG). Massime concentrazioni: 5 ind. il 18/02/1992 nella Palude della Trappola (P. Arrigucci); 3 ind. il 09/02/1998 in mare a Punta del Corvo, Talamone (GA e P. Arrigucci) (Fig. 22). Segnalato un caso di nidificazione con 2 giovani nella Palude della Trappola il 07/07/1967 (Brichetti, 1980).

\section{PHOENICOPTERIFORMES}

\section{Phoenicopteridae}

Fenicottero Phoenicopterus roseus $\mathrm{M}$ reg, W reg

In Europa, è specie nidificante in incremento (Staneva $\&$ Burfield, 2017). Con le migrazioni e lo svernamento viene rilevato in vasti complessi umidi costieri, specialmente lagune, stagni e saline; occasionale nell'interno (Brichetti \& Fracasso, 2003). Nel PRM, è accidentale nei primi anni dall'istituzione (Allavena, 1975a; Romè 1980a). La specie risente positivamente di una recente tendenza all'espansione dell'areale riproduttivo nel Mediterraneo, ed incremento dei contingenti nidificanti, tale da interessare anche la provincia di Grosseto (Baccetti et al., 1994; 2008). La Macrozona "Maremma Grossetana" è un sito di importanza regionale e nazionale per lo svernamento della specie (Arcamone et al., 2007; Zenatello et al., 2014). Oggi, nell'area protetta è osservato durante tutti i mesi dell'anno, verosimilmente anche in conseguenza delle trasformazioni ambientali che favoriscono la sosta di centinaia di individui nella più vicina Riserva Regionale Diaccia Botrona (Arcamone et al., 2007; PG). Nel periodo 2013-2017, vengono mediamente censiti 81 individui per stagione invernale (fonte: COT, archivio PRM). Rinvenuto dalla stagione 1990/91 con 1 ind. dal 31/12/1990 al 03/01/1991 nei pressi della foce del fiume Ombrone (I. Nerozzi) per quanto già nel gennaio 1967 venivano rilevati 3 individui nella Palude della Trappola, seconda segnalazione per la stagione in Toscana (Giovacchini \& Corsi, 1993). Successivamente, a partire dall'inverno 1996/97 e più marcatamente da quello 2001/02, nella Palude della Trappola e alle Macchiozze, le presenze negli anni risultano essere regolari così come si nota, grazie a ripetute letture di anelli in PVC, una tendenza per alcuni individui ad ampliare il periodo di permanenza nell'area protetta ed un effettivo incremento numerico dei contingenti, quest'ultimo sicuramente condizionato anche da erratismi, con interessanti picchi di presenza, ad es. 113 ind. il 26/07/1997 alle Macchiozze (PG); 121 ind. il 21/07/2001 alle Macchiozze (PG); 121 ind. il 05/01/2002 nella Palude della Trappola (PG); 110 ind. il 06/01/2015 nella Palude della Trappola (M. Colli e PG). Osservazioni non propriamente costiere: 45 ind. in volo il 19/11/1993 in località Collecchio (L. Bernardini); 9 ind. in volo il 29/08/1994 in località Spolverino risalgono il fiume Ombrone verso nord (G. F. Martini). Provenienza degli individui con anelli in PVC letti nel periodo 1996-2017 ( $\mathrm{n}=33)$ : 51.6\% Francia, 42,4\% Italia, $6,0 \%$ Spagna (PG, banca dati ISPRA). 


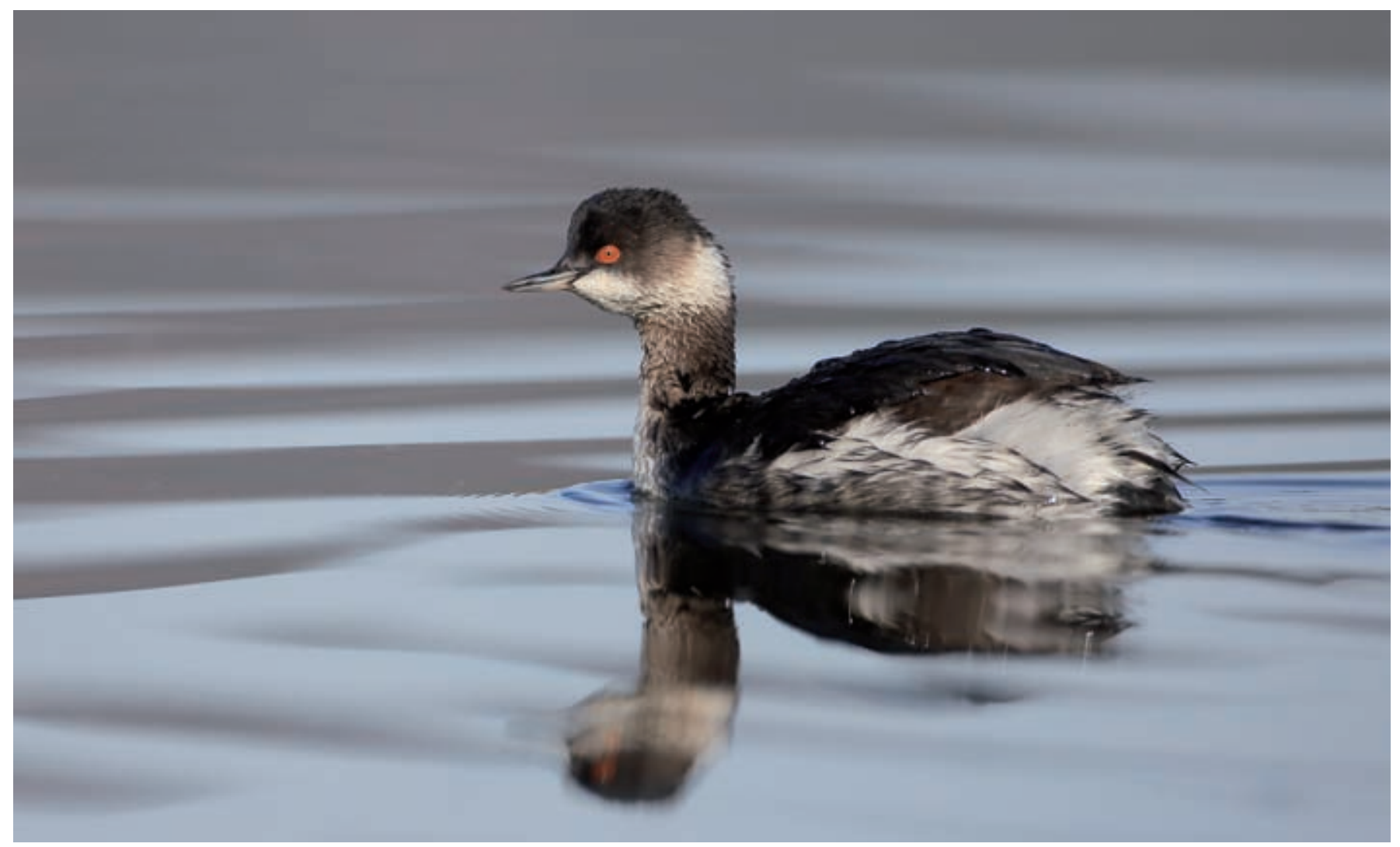

Fig. 22 - Svasso piccolo. / Black-necked Grebe (Foto / Photo Fabio Cianchi).

\section{CICONIIFORMES}

\section{Ciconiidae}

\section{Cicogna nera Ciconia nigra $\mathrm{M}$ irr}

In Europa, è specie nidificante in incremento (Staneva $\&$ Burfield, 2017). Nei periodi delle migrazioni risulta più frequente in zone aperte, anche coltivate, e nelle paludi, prati allagati e lagune (Brichetti \& Fracasso, 2003). Nel $\mathrm{PRM}$, è migratrice irregolare con un interessante numero di osservazioni raccolte sin dal 1982 che pone già da tempo l'area protetta tra i più importanti siti in Toscana (Giovacchini, 2005). Successivamente al 2004, quindi alle 12 segnalazioni recenti raccolte, se ne aggiungono altre 9 sino al 2018, riferibili unicamente a singoli individui il 75\% dei quali non adulti (GA; PG; L. Minucci; F. Ceccolini su Ornitho.it). Si tratta di osservazioni avvenute alla foce del Fiume Ombrone, Macchiozze, Spergolaia e nella Palude della Trappola, per quanto ascrivibili anche ad individui contattati in volo. Alla confermata rilevanza dell'area protetta per il numero di segnalazioni raccolte nell'intervallo 2005-2018 durante la migrazione autunnale (75\%), si aggiungono ora le osservazioni relative al periodo primaverile: 1 ind. il 22/04/2009 nella Palude della Trappola (M. Madeddu); 1 ind. il 26 e 27/05/2011 alla foce del fiume Ombrone (C. Martelli e A. Martelli in GOM-List). Una segnalazione tardo autunnale: 1 ind. il 29 e 30/11/2015 nella Palude della Trappola (PG; F. Ferragamo Visconti e G. Visconti). Inusuale la presenza di 1 ind. il 06/07/2018 in volo sulle Macchiozze (GA e L. Minucci).

\section{Cicogna bianca Ciconia ciconia $\mathrm{M}$ irr, W irr}

In Europa, è nidificante in incremento (Staneva \& Burfield, 2017). Nei periodi delle migrazioni e svernamento è osservabile in zone erbose aperte, anche intensamente coltivate, e nelle zone umide; è una specie in incremento ed espansione determinati in parte dalle reintroduzioni (Brichetti \& Fracasso, 2003). I casi di svernamento osservati più facilmente nel Centro-Nord del Paese, con riferimento quindi anche alla Toscana, possono essere ascrivibili ad individui rilasciati o sfuggiti da locali centri di allevamento della specie (Zenatello et al., 2014). Nel PRM, i dati raccolti durante l'anno risultano in parte riconducibili a loro movimenti erratici, aspetto comprovato da alcune letture di anelli in PVC attribuibili nel passato, almeno sino al 2004 circa, ad animali del Centro CARAPAX di Massa Marittima (R. Capecchi, com. pers., PG). Tale situazione sicuramente avvantaggia poi la sosta di individui di origine selvatica. Nell'area protetta sono 37 le segnalazioni catalogate nel periodo 1990-2018: queste sono specialmente riferite a singoli individui rinvenuti posati in zone aperte erbose $\mathrm{o}$ in luoghi posti in prossimità della foce del fiume Ombrone con specchi d'acqua dolce o paludi. La specie viene contattata in tutti mesi con la sola esclusione di gennaio; massima presenza in maggio con 9 dati raccolti. Vengono riscontrati pressochè identici termini di rilevanza per i soli movimenti migratori lungo questa parte della costa tirrenica $(\mathrm{n}=17$, osservazioni luglio-novembre; $\mathrm{n}=16$, febbraio-maggio). Nello stesso periodo 1990-2018, invece, assume un valore diverso la consistenza complessiva degli stessi contingenti, con una netta prevalenza di 
quelli autunnali (42 individui) rispetto ai primaverili (18 individui). Massime concentrazioni in migrazione: 6 ind. il 03/11/1990 nei pressi dei Rimessini, Marina di Alberese (A. Zampieri); 4 ind. in volo il 30/09/2014 nei pressi di Spolverino (R. Ragazzini); 2 ind. dal 15 al 19/05/2001 nelle zone della Palude della Trappola ed alla foce del fiume Ombrone (P. Arrigucci e M. Lunardi; PG). In periodo riproduttivo: 1 ind. in volo il 17/06/1997 nella zona della Palude della Trappola (P. Arrigucci). Presenze invernali nelle stagioni 2000/01 e 2001/02 con una concentrazione massima di 3 ind. il 16/12/2000 nella Palude della Trappola (L. Minucci).

\section{PELECANIFORMES}

\section{Threskiornithidae}

Ibis sacro Threskiornis aethiopicus $\mathrm{M}$ irr, $\mathrm{W}$ irr

In un recente passato risultava escluso dall'elenco delle specie dell'area protetta (Giovacchini, 2006). Per il PRM segnalazioni di 1 ind. dal mese di agosto al 12/09/2001 nei chiari della Trappola, all'interno della Tenuta di San Carlo, rilevato poi il 29/09/2001 al dormitorio di cormorani lungo il fiume Ombrone (GA; U. Bambi e R. Ragazzini). Osservato 1 ind. il 25/08/2003 nella Palude della Trappola (GA e U. Boldorini). Sino a 6 ind. il 09/12/2017 ed ancora il 16/12/2017 nella Palude della Trappola (O. Janni su Ornitho.it; PG). Della specie esotica non se ne conosce l'impatto sulla biodiversità (Andreotti et al., 2001; Baccetti et al., 2014).
Ibis eremita Geronticus eremita $\mathrm{M}$ irr

Precedentemente non rinvenuto nell'area protetta (Giovacchini, 2006). Individui riconducibili al Progetto "Waldrappteam". Le più recenti segnalazioni: 1 ind. nel settembre 2011 al Lasco di Alberese (A. Gianni); 10 ind. il 22/09/2015 alle Macchiozze (A. Gianni). Specie esclusa dalla check-list dell'avifauna italiana (Baccetti et al., 2014; Brichetti \& Fracasso, 2015b).

\section{Mignattaio Plegadis falcinellus $\mathrm{M}$ reg, W irr}

La specie risente positivamente di una recente tendenza all'incremento dei contingenti nidificanti in Europa (Staneva $\&$ Burfield, 2017). Con le migrazioni e durante lo svernamento frequenta, ad esempio, rive dei fiumi, acquitrini, prati allagati, pascoli e risaie (Brichetti \& Fracasso, 2003). Lo svernamento nel Mediterraneo risulta forse favorito dai cambiamenti climatici in atto (Zenatello et al., 2014). Risalgono alla metà degli anni ' 80 del secolo scorso le prime segnalazioni di presenza nel PRM con 1 ind. il 28/07/1986 alle Macchiozze (GA in Arcamone \& Tellini, 1987). Negli anni successivi la specie mostra un deciso incremento delle osservazioni, in particolare dall'autunno del 2000, dopo aver registrato nell'inverno 1990/91 il primo caso di svernamento per la Toscana, allora uno dei pochi nel Paese, con 1 ind. dal 08/12/1990 al 13/12/1990 ed infine 1’08/01/1991, alle Macchiozze e nella Palude della Trappola (Giovacchini, 1990a). A questo dato si aggiungono altri effettivi casi, perlomeno per le stagioni 2000/01, 2001/02, 2002/03, 2004/05 e 2017/18, verosimilmente con sole presenze invernali nel 1999/2000 e 2003/04 (Giovacchini in Arcamone \& Brichetti, 2001; M. Lausetti; PG; L. Tinti;

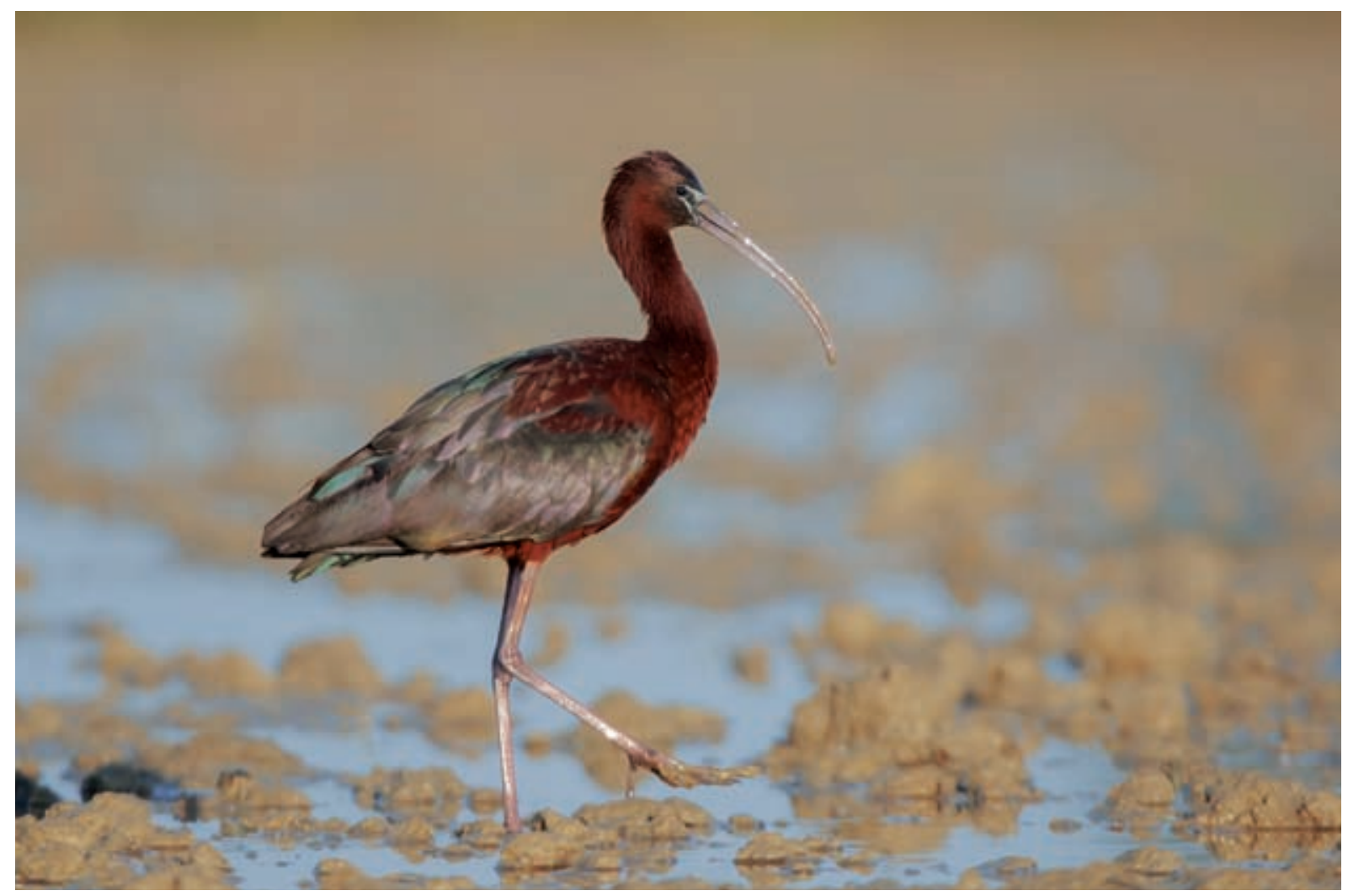

Fig. 23 - Mignattaio nelle Risaie di Principina. / Glossy Ibis in the Principina rice fields (Foto / Photo Marco Brandi). 
GA; S. Paoloni; O. Janni su Ornitho.it). Tuttavia, in taluni di questi periodi si deve anche constatare l'esistenza di movimenti pendolari di individui verso zone limitrofe all'area protetta (per esempio: cava dismessa della Voltina, inverno 2004/05), all'interno della Macrozona "Maremma Grossetana", che potrebbero indurre a valutare diversamente i risultati qui esposti. Le segnalazioni fanno principalmente capo alle risaie e alla Palude della Trappola, foce del fiume Ombrone, Macchiozze ed al dormitorio posto lungo il fiume Ombrone, località Bilancia dei Ferrovieri, con Bubulcus ibis, Ardea alba e Egretta garzetta, quest'ultimo utilizzato dalla specie perlomeno negli inverni 2001/02 e 2002/03 (GA; PG). Concentrazioni massime rilevate durante lo svernamento: 5 ind. dal 22/11/2002 al 15/01/2003 nella Palude della Trappola e al dormitorio lungo il fiume Ombrone (PG); 2 ind. dal 30/11/2004 al 15/01/2005 rinvenuti con i conteggi serali al dormitorio di Bubulcus ibis, Ardea alba e Egretta garzetta della vicina Voltina (S. Paoloni e P. Chietti) come, ugualmente, nella Palude della Trappola ad es. il 26/12/2004 (PG). Segnalazioni interessanti in periodo migratorio ed estivo: 6 ind. il 27/04/1998 nella Palude della Trappola (M. Lunardi e A. Gianni); 50 ind. il 24/04/2002 alla foce del fiume Ombrone (GA e PG); almeno 30 ind. il 11/05/2002 alla foce del fiume Ombrone (PG); 3 ind. il 08/06/2011 nelle Risaie della Trappola (A. Gianni); 12 ind. il 17/06/2016 nelle Risaie di Principina, immediatamente fuori dal PRM (M. Colli in GOM-List) (Fig. 23).

\section{Spatola Platalea leucorodia M reg, W reg}

Specie in incremento in Europa come nidificante (Staneva \& Burfield, 2017). In Italia, mostra da tempo un'espansione dell'areale riproduttivo, frequentando durante le migrazioni e lo svernamento zone umide costiere (Brichetti \& Fracasso, 2003). La Macrozona "Maremma Grossetana" è un sito di importanza regionale e nazionale per lo svernamento della specie (Arcamone et al., 2007; Zenatello et al., 2014). Nel PRM, le presenze assumono regolarità dal 2011. Risalgono al precedente decennio le prime indicazioni di presenza nel periodo invernale (Monti, 2012a). La specie frequenta la Palude della Trappola e gli specchi interni delle Macchiozze, più raramente la zona di foce del fiume Ombrone. Recentemente è stata rilevata con concentrazioni a volte interessanti per il sito: 16 ind. insieme il 08/01/2016 alle Macchiozze (PG). Il 17/01/2016 censiti complessivamente 29 individui svernanti nel PRM (fonte: COT, archivio PRM). Le osservazioni realizzate in periodo primaverile negli anni evidenziano una consistenza numerica più contenuta di quelle raccolte nel periodo autunnale, ma comunque sempre con interessanti concentrazioni, se riferite all'area di studio: massimo 7 ind. insieme il 28/03/1994 alla foce del fiume Ombrone (L. Buratta e PG). Le poche letture di anelli in PVC $(n=5)$ indicano attualmente una prevalenza di individui nati in Italia ed un solo animale proveniente dall'estero (Ungheria) (PG, banca dati ISPRA).

\section{Ardeidae}

Tarabuso Botaurus stellaris M reg, W irr?

In Europa, è nidificante stabile (Staneva \& Burfield, 2017). Con le migrazioni e lo svernamento frequenta an- che rive dei fiumi, canali, fossati, piccoli stagni e margini di paludi salmastre (Brichetti \& Fracasso, 2003). Nel PRM, le informazioni raccolte inducono a proporre uno status incerto come svernante, così come ogni stima di abbondanza potrebbe non delineare, dato il comportamento elusivo della specie, la reale portata dello svernamento che, quando osservato, è quasi sempre limitato a 1-2 individui (Romè, 1982; Arcamone et al., 2007). A testimonianza della difficoltà nel rinvenire la specie sono alcune segnalazioni raccolte in stagioni invernali in cui, al contrario, non compare con i censimenti di gennaio dello stesso anno, ad es. 1 ind. il 09/01/1994 nella Palude della Trappola (GA); 1 ind. il 26/01/1996 ai margini del Chiaro Grande nella Padule della Trappola (GA e L. Minucci); 1 ind. il 18/01/2017 nella Palude della Trappola (PG). La Macrozona "Maremma Grossetana" è tuttavia indicata come sito di importanza regionale per lo svernamento della specie (Arcamone et al., 2007). Gli ambienti dell'area protetta non sembrano idonei per la riproduzione, soprattutto in assenza di vasti canneti con acqua dolce. Dalla metà di ottobre alla prima quindicina di aprile, oltre che per le zone indicate, si hanno occasionali segnalazioni di singoli individui anche in migrazione ai margini della vegetazione elofitica di corsi d'acqua di piccole dimensioni, come i canali Essiccatore e Perazzeta dell'Alberese, così come ai bordi degli specchi d'acqua dolce posti alle Macchiozze e a volte lungo la strada per Marina di Alberese (L. Buratta; GA e L. Minucci; L. Bernardini; PG; M. Lunardi e A. Gianni; D. Germani; M. Porciani; P. Arrigucci). Si segnala 1 ind. il 06/07/1997 nelle vicine Risaie di Principina Mare (G. Bencivenga).

Tarabusino Ixobrychus minutus M reg, B irr

In Europa, è nidificante stabile (Staneva \& Burfield, 2017). In Italia, è nidificante "Vulnerabile" (Peronace et al., 2012). Nidificante in zone umide di acqua dolce, naturali o artificiali, anche di ridotte dimensioni, con vegetazione diversificata, dal livello del mare a 300-400 m s.l.m. circa; con le migrazioni frequenta anche ambienti coltivati, centri abitati e aree montane (Brichetti \& Fracasso, 2003). Nidificante irregolare nella vicina Riserva Regionale Diaccia Botrona (Giovacchini \& Stefanini, 2015). Specie dal comportamento elusivo, con la migrazione primaverile che nel PRM, rispetto a quella autunnale, appare essere regolare e verosimilmente limitata a pochi individui. L'osservazione è confermata dal fatto che solo una frazione delle popolazioni dell'Europa centrale attraversa la penisola italiana in autunno (Brichetti, 1985; 1992). Nell'area protetta, la mancanza di un adeguato ed esteso habitat riproduttivo costituito da canneti inondati comporta l'osservazione della specie più facilmente lungo il fiume Ombrone e sul canale Essiccatore, nonchè in misura minore sulle rive di piccoli bacini di origine artificiale posti nella zona nord, come il laghetto del Voltone nella Azienda Agricola di San Mamiliano e, nello stesso ambito, nell'area del collettore Morelle. Specialmente dal 2000 , viene rilevata la presenza con singoli individui lungo il fiume nei pressi della località Bilancia dei Ferrovieri, ove la vegetazione elofitica ripariale associata a quella arborea appare a tratti piuttosto densa, sebbene limitata nell'estensione (GA; PG). Si segnala la riproduzione 


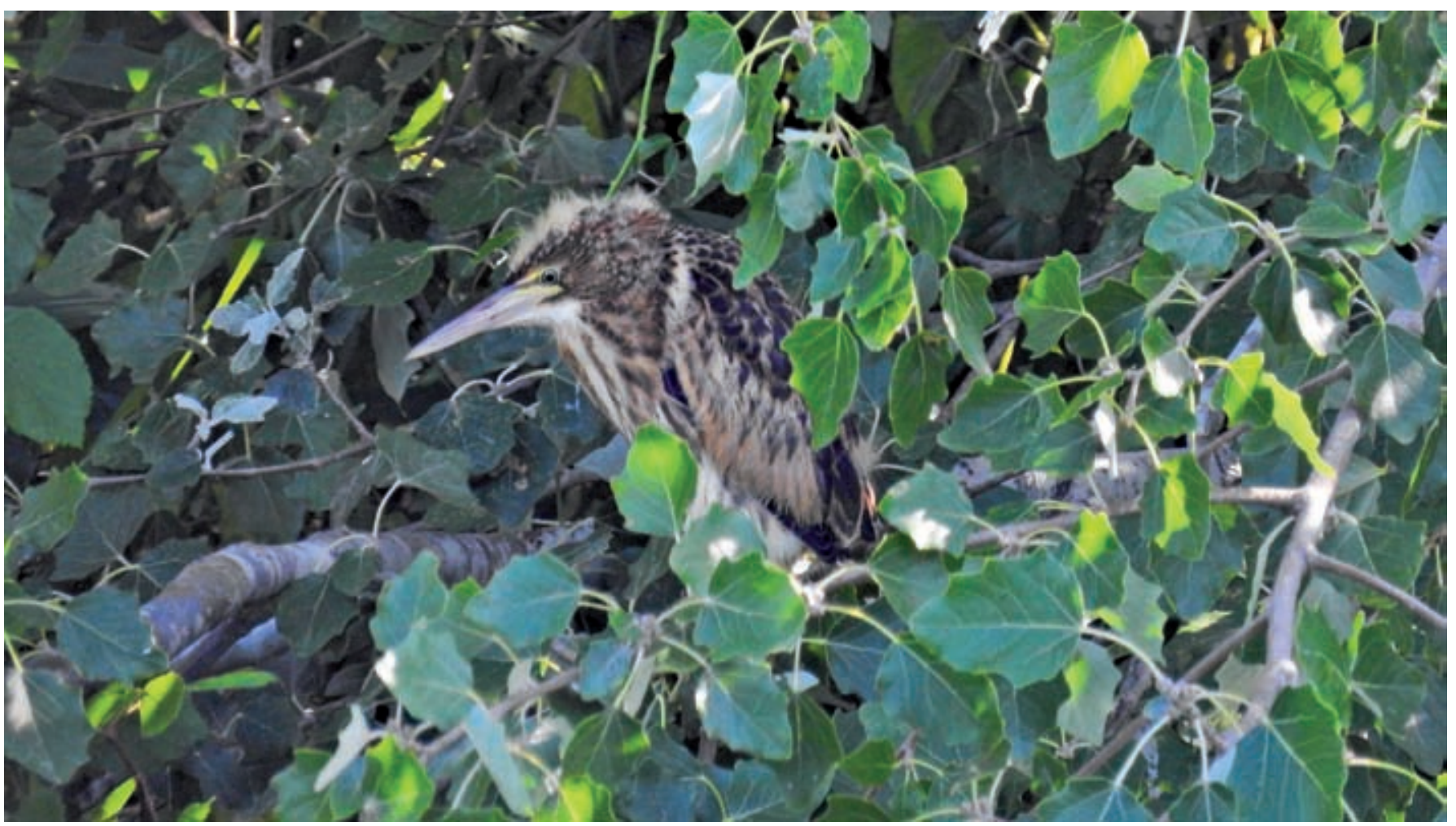

Fig. 24 - Giovane di tarabusino il 27/06/2017, Pian di Barca, fiume Ombrone. / Young Little Bittern on the $27^{\text {th }}$ June 2017 in the Pian di Barca locality, Ombrone river (Foto / Photo Vincenzo Rizzo Pinna).

certa di una coppia nel laghetto del Voltone a San Mamiliano: 1 femmina ed 1 giovane non ancora in grado di volare il 27/06/2002, ai limiti della vegetazione spondale (PG); 1 femmina il 09/07/2002 nello stesso luogo (PG). Nuovo caso di nidificazione con 1 giovane non ancora in grado di volare il 27/06/2017, lungo il fiume Ombrone nei pressi di Pian di Barca (V. Rizzo Pinna; Fig. 24).

Nitticora Nycticorax nycticorax $\mathrm{M}$ reg, W irr?

In Europa, è nidificante in decremento (Staneva \& Burfield, 2017). Durante le migrazioni frequenta ambienti acquatici disparati, anche marini costieri e montani; in svernamento, ad esempio, rive boscose di fiumi, canali, valli da pesca (Brichetti \& Fracasso, 2003). Le informazioni raccolte nel PRM, specialmente negli ultimi dieci anni, consentono di descrivere la fenologia della specie con una certa precisione permettendo di attribuire alcune delle assenze registrate in periodo invernale come possibili carenze nei rilievi per una specie dal comportamento elusivo e mobile. Gli erratismi ed i ripetuti avvistamenti di singoli individui estivanti, registrati nell'area protetta almeno dal 1986, potrebbero fare riferimento anche ad adulti non riproduttori, forse gravitanti nelle colonie toscane confinate nel nord della regione (Fasola \& Alieri, 1992; Battaglia \& Sacchetti, 1997). È certamente significativo il ruolo dell'area di studio per le finalità trofiche, specialmente nella zona del fiume Ombrone. Dopo l'inverno 2004/05 la specie viene successivamente rilevata nelle stagioni 2005/06, 2011/12, 2013/14, 2015/16 e 2017/18. Sono riferibili ai conteggi al dormitorio della cava dismessa della Voltina, ma spesso anche a contestuali segnalazioni registrate nell'area protetta, $\mathrm{i}$ dati raccolti nella stagione 2011/12: 2 ind. il 22/12/2011 alla Voltina (S. Paoloni); 5 ind. il 06/01/2012 in volo serale alle Cetine, Trappola (PG e F. Farsi); inverno 2013/14: 2 ind. il 21/12/2013 lungo la strada per Marina di Alberese (G. Radi, G. Bencini, PG, M. Porciani; Fig. 25); 1 ind. il 30/12/2013 alla Voltina (PG e P. Fastelli). Massima concentrazione invernale: 8 ind. il 27/12/2017 in volo alle Cetine, provenienti da un dormitorio posto immediatamente fuori dal PRM, vicino alle Risaie di Principina, utilizzato irregolarmente almeno dall'inverno 2011/12 (PG). Rinvenuto per la prima volta come invernale nell'area protetta con 1 ind. in volo il 24/01/1995 a Spolverino (GA). Frequentazione saltuaria di tratti del fiume Ombrone come dormitori, a cominciare con certezza dall'inverno 1996/97: 3 ind. (tutti immaturi) il 08/12/1996 in località Bilancia dei Ferrovieri (PG). Successiva presenza nello stesso periodo dell'anno: 2 ind. in richiami il 10/12/1998 a Principina Mare (PG). Concentrazioni significative in periodo migratorio: 21 ind. (17 immaturi e 4 adulti) il 25/09/2002 tra la vegetazione alla foce del fiume Ombrone (PG); circa 30 ind. (adulti e immaturi) il 15/06/2011 a Pian di Barca (R. Ragazzini).

Sgarza ciuffetto Ardeola ralloides M reg

In Europa, è nidificante stabile (Staneva \& Burfield, 2017). Durante le migrazioni frequenta vari tipi di zone umide costiere ed interne (Brichetti \& Fracasso, 2003). Nidifica nella vicina garzaia della pineta delle Marze, all'interno della Riserva Regionale Diaccia Botrona ( $\mathrm{Pu}-$ glisi et al., 2012). I casi di estivazione di adulti, registrati con maggiore regolarità dal 2003, fanno anche riferimen- 


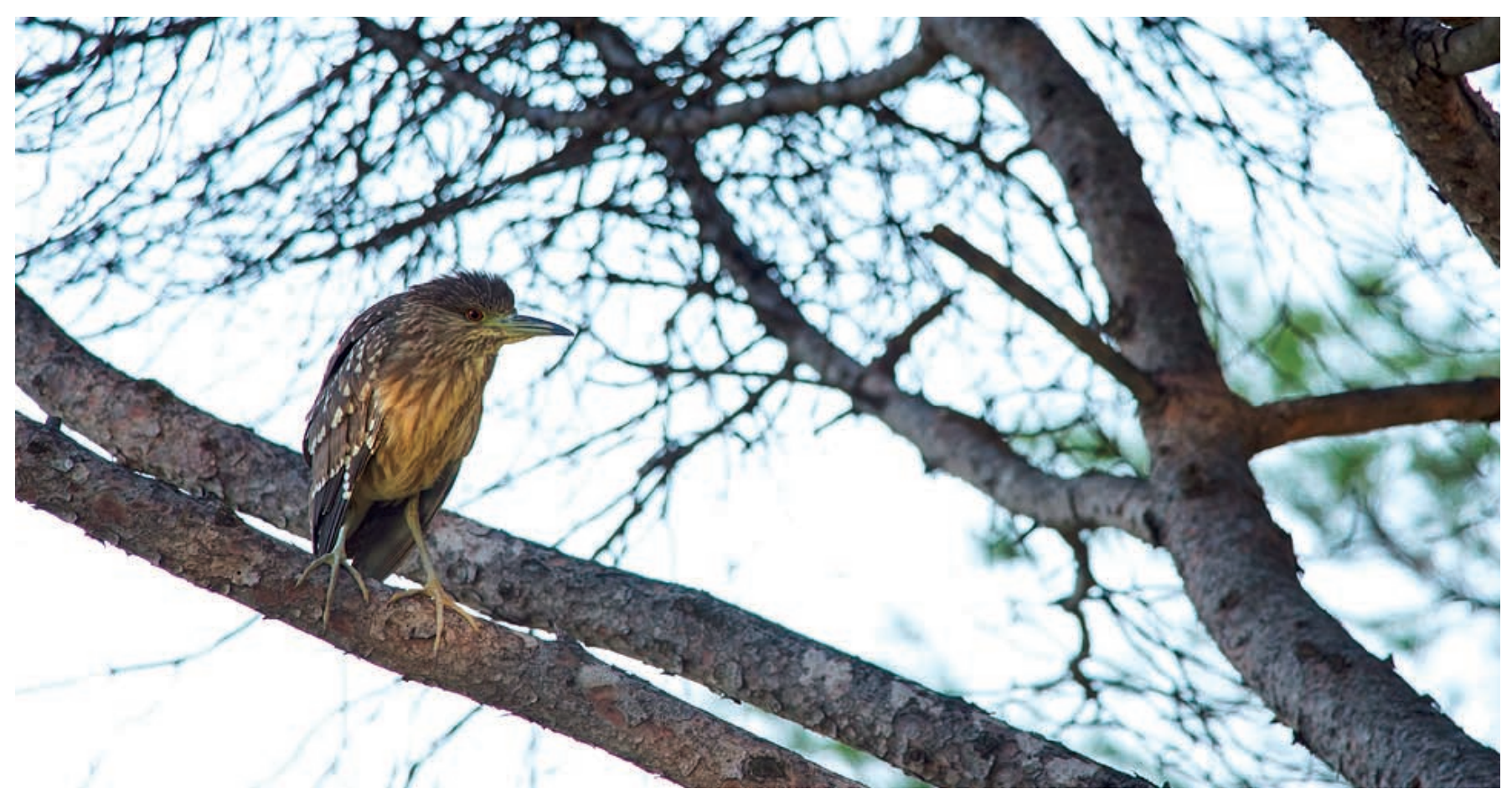

Fig. 25 - Nitticora il 21/12/2013 nei pressi dei Rimessini di Alberese. / Night Heron on the 21 ${ }^{\text {st }}$ December 2013 near Rimessini, Alberese (Foto / Photo Giacomo Radi).

to ad osservazioni che interessano le Risaie di Principina, generalmente con un numero non superiore ai 3-5 individui (M. Colli; F. Farsi). Occorre tuttavia precisare che le presenze legate al mese di giugno potrebbero essere ancora imputabili a movimenti migratori in atto così come a spostamenti tra aree limitrofe, verosimilmente riconducibili ad individui provenienti da garzaie vicine (Dragonetti \& Giovacchini, 2009; Giovacchini \& Stefanini, 2015). Nell'area di studio vengono utilizzati i pascoli cespugliati della Palude della Trappola, e di confine per il PRM a Principina Mare, più facilmente in presenza di piccole aree allagate, così come le zone antistanti alla foce del fiume Ombrone. Prima segnalazione come estivante: 1 ind. dal 01 al 12/07/1996, verosimilmente lo stesso tra San Mamiliano, Trappola, e la foce del fiume Ombrone (GA; P. Arrigucci; PG). Frequentazione più recente: 1 ind. il 08/07/2017 strada del mare, Tenuta di Torre Trappola (PG).

\section{Airone guardabuoi Bubulcus ibis $\mathrm{M}$ reg, $\mathrm{W}$ reg}

Specie in decremento in Europa come nidificante (Staneva \& Burfield, 2017). Con lo svernamento frequenta anche acquitrini, prati allagati, marcite, campi arati, pascoli, centri urbani (Brichetti \& Fracasso, 2003). Nidifica dal 1999 nella più vicina garzaia posta nella pineta delle Marze, all'interno della Riserva Regionale Diaccia Botrona (Giovacchini et al., 2001). La prima segnalazione nel PRM fa riferimento ad una osservazione tardo-autunnale: 1 ind. il 29/11/1982 alla foce del fiume Ombrone (Corsi in Toso, 1983). Per quanto attualmente non nidificante nell'area protetta, viene rilevato più facilmente da fine marzo sino ad ottobre-inizio novembre, per poi ricomparire a dicembre sino ad inizio febbraio con contingenti numericamente diversificati negli anni, più ingenti nella fase legata alla dispersione post riproduttiva ed alla migrazione autunnale. Nel periodo 2013-2017 è segnalato con un massimo di 16 individui svernanti (fonte: COT, archivio PRM). La specie mostra nell'area protetta una forte preferenza per l'alimentazione nei pascoli frequentati da bovini ed equini mantenendo una stretta associazione con le mandrie mentre si nutrono (Paoloni et al., 2018). Condizioni climatiche rigide possono comunque indurre gli uccelli a repentini spostamenti di luogo (Passalacqua et al., 2015). Per quanto limitatamente esterno all'area considerata, il dormitorio della Voltina riconferma negli anni la sua importanza come centro di aggregazione notturno per individui provenienti anche da comprensori più ampi o in migrazione: sino a 561 ind. il 10/10/2006 (S. Paoloni). Sebbene con numeri fluttuanti, forse anche per l'esistenza contemporanea del precedente, i dormitori su Populus sp. posti nell'area protetta lungo il fiume Ombrone risultano quantomeno utilizzati nelle stagioni 2000/01 e 2001/02: 86 ind. il 11/01/2001 a Pian di Barca (GA); 2 ind. il 05/01/2002 in località Bilancia dei Ferrovieri (PG), con Plegadis falcinellus ed Egretta garzetta. Nella Palude della Trappola, i censimenti realizzati dall'inverno 2007/08 al 2014/15 nel dormitorio di ardeidi su Pinus pinea hanno permesso di rilevare il 14/12/2014 un massimo di 36 individui insieme ad Ardea alba ed Egretta garzetta (GA, M. Anselmi e M. Simoni; Passalacqua et al., 2015). Alla colonizzazione della garzaia delle Marze potrebbero fare riferimento alcune segnalazioni registrate nel PRM e relative ad antecedenti periodi riproduttivi: 1 ind. fine giugno 1985 nella Palude della Trappola (GA in Arcamone \& Tellini, 1986); più recentemente, 2 ind. il 11/05/1995 nel Lasco di Alberese (M. Marinelli); 10 ind. 
il 30/05/1997 nel Lasco di Alberese (PG). L'osservazione di 2 giovani da poco involati in un gruppo di 31 ind. il 06/07/1998 alle Macchiozze (GA e PG) potrebbe fare anticipare al 1998 l'anno della prima riproduzione in provincia di Grosseto. Anche l'inverno 1996/97 fornisce un dettaglio, con il primo caso di presenza invernale per l'intera pianura grossetana: 1 ind. dal 2 al 03/01/1997 nella Palude della Trappola (PG).

\section{Airone cenerino Ardea cinerea SB, M reg, W reg}

Specie in decremento in Europa come nidificante (Staneva \& Burfield, 2017). Nidificante su alberi, arbusti e vegetazione palustre, più diffuso sino a $250 \mathrm{~m}$ s.l.m.; con le migrazioni ed in inverno è più comune in zone umide di acqua dolce o salmastra, laghi, bacini artificiali, litorali, fiumi, canali, coltivi (Brichetti \& Fracasso, 2003). Nel $\mathrm{PRM}$, è specie rilevata durante tutto l'anno, più spesso con singoli individui, nelle zone aperte con terreni incolti, pascoli, risaie, prati, ma anche lungo i corsi d'acqua ed in corrispondenza della foce del fiume Ombrone così come ai margini delle zone umide d'acqua dolce o salmastra. Nel periodo 2013-2017 la popolazione svernante nell'area protetta è stata mediamente valutata in circa 30 individui (fonte: COT, archivio PRM). La Macrozona "Maremma Grossetana" è un sito di importanza regionale per lo svernamento della specie (Arcamone et al., 2007). Le cifre che scaturiscono dai censimenti per stagione rappresentano verosimilmente soltanto una frazione del contingente gravitante ogni giorno nell'area, specialmente tenedo conto della buona diffusione della specie lungo la costa grossetana. La riproduzione viene indicata, seppur senza prove dirette, come possibile nella zona della Trappola sino al 1970 circa (Romè, 1983a). Più recentemente, indagini condotte con approcci diversi testimoniano per l'intera provincia un deciso incremento della consistenza di popolazione (Puglisi et al., 2012; Giovacchini et al., 2017). Nell'area protetta, le conferme iniziano dalla stagione 2002 quando una coppia ha nidificato a Campo al Pino, nei pressi della foce del fiume Ombrone, su una pianta di Pinus pinea (PG; Fasola et al., 2007). Un nuovo caso di riproduzione viene osservato nel 2004 al quale fa seguito, nel 2005, il fallimento di una coppia insediatasi nella stessa località dei precedenti (GA; PG). Occorre attendere il 2012 per rilevarne nuovamente la nidificazione, sempre con una coppia isolata (F. Pezzo). Nel periodo 2015-2018 prende definitivamente consistenza la garzaia: la riproduzione risulta regolare con un lieve incremento nel 2016 quando, il 18/05/2016, sono rinvenuti 6 nidi collocati su 2 piante di Pinus pinea (G. F. Martini, GA, PG). Nel 2017 e 2018 sono rispettivamente 4 e 5 le coppie nidificanti (PG).

\section{Airone rosso Ardea purpurea $\mathrm{M}$ reg}

In Europa, è in decremento come nidificante (Staneva $\&$ Burfield, 2017). Durante le migrazioni frequenta zone umide costiere ed interne ricche di vegetazione emergente; localmente, litorali marini e vallate alpine (Brichetti \& Fracasso, 2003). Osservato nel PRM da marzo in poi, generalmente sino ad aprile-inizio maggio, facilmente con singoli individui nelle Risaie della Trappola e di Principina, foce del fiume Ombrone così come ai margini delle zone umide d'acqua dolce poste specialmente alle Mac- chiozze. Occasionale altrove. L'area protetta costituisce un sito di scarsa importanza per la specie, in particolare per la migrazione autunnale dal momento che questa è apparentemente meno consistente sul Tirreno (Barbieri \& Brichetti, 1992). Presenze estive limitate, ad es. 3 ind. insieme il 12/07/1997 alle Macchiozze (PG); 1 ind. il 04/06/2007 alle Risaie di Principina (PG). Si segnala in migrazione: 7 ind. insieme il 28/03/2012 in volo diurno sopra Talamone, lungo la dorsale dei Monti dell'Uccellina (PG in GOM-List).

\section{Airone bianco maggiore Ardea alba $\mathrm{M}$ reg, W reg}

Specie in incremento in Europa come nidificante (Staneva \& Burfield, 2017). Con le migrazioni e lo svernamento frequenta preferibilmente lagune, valle da pesca e saline, risaie, prati e campi arati (Brichetti \& Fracasso, 2003). Nidificante certo da alcuni anni nella più vicina garzaia delle Marze, all'interno della Riserva Regionale Diaccia Botrona, con una popolazione che è in leggero incremento (Giovacchini \& Stefanini, 2015; Giovacchini et al., 2017). Su scala regionale, l'abbondanza invernale indica uno scenario improntato all'aumento dei contingenti, specialmente dopo la stagione 1991/92, confermando la ripresa numerica registrata per l'intero Paese (Baccetti \& Massi, 1992; Pezzo, 1997a). La Macrozona "Maremma Grossetana" è sito di importanza regionale e nazionale per lo svernamento (Arcamone et al., 2007; Zenatello et al., 2014). Nel PRM, frequenta preferibilmente terreni incolti, prati parzialmente allagati, risaie ed anche i corsi d'acqua, in corrispondenza della foce del fiume Ombrone così come i margini delle zone umide d'acqua dolce o salmastra, da nord a sud. Le cifre che scaturiscono dai più recenti censimenti confermano le favorevoli condizioni ecologiche del territorio: negli anni 2014-2017 la popolazione svernante nell'area protetta è stata mediamente valutata in 28 individui (fonte: COT, archivio PRM). Dormitori su Populus sp. posti lungo il fiume Ombrone, spesso utilizzati in contemporanea con quello collocato all'interno della cava dismessa della Voltina (GA; F. Corsi; PG; S. Paoloni e P. Chietti), risultano occupati quantomeno nelle seguenti stagioni: inverno 1996/97 con 20 ind. il 24/12/1996 (PG) e 1 ind. il 27/01/1997 a Pian di Barca (PG), con Egretta garzetta; inverno 2001/02 con 1 ind. il 05/01/2002 in località Bilancia dei Ferrovieri (PG), con Plegadis falcinellus, Bubulcus ibis ed Egretta garzetta. Nella Palude della Trappola censimenti realizzati dall'inverno 2007/08 al 2014/15 nel dormitorio di ardeidi su Pinus pinea hanno permesso di rilevare il 22/12/2008 un massimo di 34 individui insieme a Bubulcus ibis ed Egretta garzetta (GA; Passalacqua et al., 2015). Le osservazioni sulle migrazioni collocano tali movimenti nel periodo fine settembre - metà maggio con le prime osservazioni di individui estivanti nell'area protetta, tutti immaturi, che fanno riferimento al biennio 1997-1998: 2 ind. il 26/07/1997 alle Macchiozze (PG); 1 ind. il 06/06/1998 a Principina Mare (PG). Concentrazione elevata in periodo migratorio: 90 ind. circa in volo il 20/10/1994 a Principina Mare (PG). Individuo inanellato: 1 ind. catturato il 24/04/1936 nella Palude della Trappola, era stato inanellato il 28/05/1935 nella Riserva della Camargue-Bouches du Rhône, Francia (Moltoni, 1976; Bendini \& Spina, 1983). 


\section{Garzetta Egretta garzetta $\mathrm{M}$ reg, W reg}

Specie in decremento in Europa come nidificante (Staneva \& Burfield, 2017). Durante le migrazioni e lo svernamento predilige acque salmastre costiere, frequentando anche coltivi, marcite, acque urbane (Brichetti \& Fracasso, 2003). Da oltre trenta anni nidifica nella più vicina garzaia posta nella pineta delle Marze, all'interno della Riserva Regionale Diaccia Botrona (Corsi \& Giovacchini, 1995b; Puglisi et al., 2012). La Macrozona "Maremma Grossetana" è sito di importanza regionale per lo svernamento (Arcamone et al., 2007). Nel passato la riproduzione viene indicata per la zona della Trappola per quanto poi messa in discussione e comunque mai provata (Di Carlo \& Heinze, 1975; Romè, 1981). Nel PRM, frequenta preferibilmente i margini dei corsi d'acqua, per esempio in corrispondenza della foce del fiume Ombrone, così come le zone umide d'acqua dolce o salmastra, nonchè i prati parzialmente allagati e le risaie. I più recenti censimenti invernali realizzati negli anni 2014-2017 indicano per l'area protetta una popolazione valutata mediamente in 34 individui (fonte: COT, archivio PRM). Dormitori rilevati nel passato su Populus sp., e posti lungo il fiume Ombrone, vengono utilizzati in contemporanea con quello della cava dismessa della Voltina (GA; PG; S. Paoloni e P. Chietti; F. Corsi), risultando occupati perlomeno nelle seguenti stagioni: inverno 1996/97 con 60 ind. il 24/12/1996 (PG) e 25 ind. il 27/01/1997 a Pian di Barca (PG), con Ardea al$b a$; inverno 2001/02 con 47 ind. il 05/01/2002 in località Bilancia dei Ferrovieri (PG), con Plegadis falcinellus, Bubulcus ibis ed Ardea alba. Nella Palude della Trappola censimenti realizzati dall'inverno 2007/08 al 2014/15 nel dormitorio di ardeidi su Pinus pinea hanno permesso di rilevare il 17/01/2015 un massimo di 93 individui insieme a Bubulcus ibis ed Ardea alba (PG; Passalacqua et $a l ., 2015)$. Le osservazioni sulle migrazioni della specie conducono nuovamente a rilevare l'interesse dei dormitori come centro di aggregazione per individui verosimilmente in spostamento: 98 ind. il 24/10/1996 a Pian di Barca (PG). In questi periodi, rilevanti concentrazioni in aree aperte, anche in fase di dispersione: 150 ind. il 09/10/1998 nei prati della Tenuta di San Carlo (PG); 75 ind. il 11/07/2014 alle Macchiozze (PG).

\section{Airone schistaceo Egretta gularis A-2}

Accidentale. 1 ind. il 16/09/1990 nella Palude della Trappola (L. Bernardini e PG in Arcamone \& Barbagli, 1995-1996); 1 ind. il 24/09/2002 alla foce del fiume Ombrone (PG).

\section{Pelecanidae}

\section{Pellicano comune Pelecanus onocrotalus A-2}

Accidentale. 1 ind. il 03/02/1996 alle Macchiozze (fonte: Azienda Terre Regionali Toscane, A. Zampieri) (Arcamone \& Brichetti, 1997; Giovacchini, 1999). Si aggiunge ora la seguente segnalazione: 1 ind. dal 30/10 al 01/11/2002 nella Palude della Trappola e a Principina Mare (E. Calvario, S. Sarrocco e P. Sposimo in EBNItalia; B. Perroud e F. Perroud in EBN-Italia). Verosimilmente lo stesso individuo poi osservato il 02/11/2002 nella Riserva Regionale Diaccia Botrona (GA, ined.). Dubbia la provenienza (Baccetti et al., 2014).

\section{Pellicano riccio Pelecanus crispus A-1}

Accidentale. 1 ind. il 24/04/1986 alle Macchiozze e nella Palude della Trappola (L. Buratta e G. Stellini in Arcamone \& Tellini, 1987).

\section{SULIFORMES}

\section{Sulidae}

Sula Morus bassanus $\mathrm{M}$ reg, $\mathrm{W}$ reg

In incremento in Europa come nidificante (Staneva \& Burfield, 2017). Uccello strettamente pelagico, ovvero di ardua rilevabilità dalla riva, nel Paese è di recente acquisizione tra i nidificanti (Brichetti \& Fracasso, 2015b). Effettivi difficilmente quantificabili, se non forse con sforzo di indagine costante, e comunque su tratti di costa più ampi del solo litorale compreso tra Principina Mare e Talamone. Per quanto la specie compia ampi movimenti erratici a lungo raggio, all'interno del Mediterraneo ancora oggi poco noti, potrebbe essere necessario riconoscere a questo tratto di costa un'importanza diversa legata alle mutate condizioni di pescosità. Le indicazioni di presenza appaiono poi per essere distribuite nel corso dell'anno con modalità differenti rispetto ad un recente passato, se riferite (seppur su scala più ampia) al Tirreno centrale (Spanò \& Baccetti, 1992). In particolare, le segnalazioni radunate per il PRM nel periodo 1986-2017 $(n=54)$, e specialmente dopo il 1990, sono principalmente il risultato dell'incremento degli osservatori: la parte dei soli dati invernali (dicembre-gennaio) è comunque riconducibile al più elevato numero di informazioni raccolte $(n=25)$. Entrambi gli aspetti possono quindi assumere un importante ruolo per una maggiore conoscenza della fenologia della specie. Le segnalazioni sono più facilmente ascritte a singole presenze o a gruppi di 2-3 individui. Rari i casi concernenti gruppi numerosi: 20 ind. il 21/04/1991 alla foce del fiume Ombrone (D. Occhiato in Arcamone \& Barbagli, 1995-1996). Occasionale in periodo estivo: 1 immaturo il 15/06/1997 in vicinanza della costa dei Monti dell'Uccellina (GA); 1 ind. il 19/06/2009 alla foce del fiume Ombrone (F. Monti, com. pers.; Monti, 2012a); 1 immaturo il 09/07/2012 alla foce del fiume Ombrone (S. Bassi su Ornitho.it).

\section{Phalacrocoracidae}

Marangone dal ciuffo Phalacrocorax aristotelis M reg, W irr

In Europa, è in decremento come nidificante (Staneva $\&$ Burfield, 2017). In inverno gli adulti sono spesso già insediati nelle colonie di nidificazione (Brichetti \& Fracasso, 2003). Pelagico, frequenta anche le coste rocciose purché riparate e pescose. Sino ad un miglio circa dal PRM le osservazioni fanno quasi sempre capo a singoli (o al massimo tre) individui, più spesso immaturi rilevati in volo o in acqua, anche piuttosto vicini alla linea di riva, o posati sulle scogliere dei Monti dell'Uc- 
cellina. In questo ambito dell'area protetta le prime indicazioni di presenza sono riferite alla metà circa degli anni '90 del secolo scorso: 1 ind. adulto il 05/07/1994 posato sulle scogliere a nord di Talamone (Giovacchini, 1999). Rinvenuto sicuramente con maggiore frequenza dal 2008, da Capo d'Uomo alle Cannelle, Talamone, ed anche in aree immediatamente esterne come in località Bagno delle Donne (GA). Dispersivo, compie migrazioni a corto raggio, testimoniando una importante, per quanto limitata, presenza come nidificante sulle isole ed isolotti del Tirreno centrale, specialmente dopo il 1980 (Brichetti et al., 1992). In questo senso, i dati raccolti nel PRM potrebbero risentire del calendario delle deposizioni che a queste latitudini decorre da febbraio, trovando così un'ulteriore possibile spiegazione il limitato numero di osservazioni riferite al periodo invernale (Brichetti, 1982).

\section{Cormorano Phalacrocorax carbo $\mathrm{M}$ reg, $\mathrm{W}$ reg}

In Europa, è specie nidificante in incremento (Staneva $\&$ Burfield, 2017). Sverna in acque dolci o salmastre, dalle lagune ai bacini artificiali montani e urbani, con abbondanti ed accessibili risorse alimentari (Brichetti \& Fracasso, 2003). Notoriamente piscivoro, nel PRM viene più facilmente osservato in attività trofica, occasionalmente anche in gruppi numerosi (cd. "pesca cooperativa" o "pesca sociale"), o posato su tronchi, alberi, paletti o bricole, lungo il corso del fiume Ombrone ed alla foce, così come nella Palude della Trappola e nei canali di bonifica distribuiti da nord a sud. Scarso sul mare. L'area protetta, tuttavia, nel panorama regionale è nota per essere uno dei siti secondari per la specie, stabilmente occupato in ogni stagione di svernamento, con frequenti casi di individui osservati in pendolarismo per alimentazione, anche da aree esterne (Arcamone et al., 2007). Alla situazione registrata nei primi anni ' 80 del secolo scorso, ha poi fatto seguito il riscontro di comportamenti diversi dal precedente decennio, anche con la colonizzazione di un tratto della vegetazione arborea ripariale su Populus sp. eletto a dormitorio dall'inverno 1990-91 (Baccetti, 1989; Giovacchini, 1994). L'esame delle borre raccolte negli anni ha permesso di appurare che il $90 \%$ della dieta della specie è costituita da Mugilidae (Martucci \& Giovacchini, 1994). La popolazione gravitante nel nucleo di Bocca d'Ombrone, per quanto spesso riferita a conteggi non riconducibili ai dormitori, nel periodo 2013-2017 risulta mediamente attestata in 145 individui svernanti l'anno (fonte: COT, archivio PRM). Massima presenza negli anni al dormitorio serale, lungo il corso del fiume Ombrone: 392 ind. il 13/01/2002 (PG). Letture di anelli in PVC con codici alfanumerici e riscontro di un caso di fedeltà al sito di svernamento: 1 ind. il 19/11/1994, 05/11/1995, 09/01/1996, 23/10/1996, 17/11/1996, al dormitorio lungo il corso del fiume Ombrone, era stato inanellato il 28/05/1990 a Toft, Vorsø, Danimarca (fonte: National Environmental Research Institute, Horsens, Denmark; PG); 1 ind. il 26/10/1996, al dormitorio lungo il corso del fiume Ombrone, era stato inanellato il 02/06/1996 a Vorsø, Danimarca (fonte: National Environmental Research Institute, Horsens, Denmark; PG).

\section{ACCIPITRIFORMES}

\section{Pandionidae}

Falco pescatore Pandion haliaetus $\mathrm{M}$ reg, W reg, B

Specie in incremento in Europa come nidificante (Staneva \& Burfield, 2017). Durante le migrazioni osservato in vari ambienti umidi costieri e interni; lo svernamento interessa lagune e stagni costieri nonchè, localmente, laghi artificiali interni (Brichetti \& Fracasso, 2003). Il PRM è specie oggetto di interessanti azioni intraprese sin dal 2004, e tutt'ora in corso, nell'ambito del più vasto "Progetto Falco pescatore", inizialmente promosso e sostenuto soltanto dallo stesso Ente Parco e dal Parco Naturale Regionale della Corsica, per la ricostituzione di una popolazione della specie nel Paese e per agevolare l'espansione di quella corsa. In particolare, dal 2006 sono state attivate traslocazioni in Maremma di giovani individui prelevati dai nidi nella Riserva Naturale di Scandola, Corsica, Francia, adottando la tecnica dell'hacking e, successivamente, l'uso di strumentazione satellitare per una analisi della dispersione degli individui rilasciati e della filopatria (Monti et al., 2018). Il primo caso di riproduzione nell'area protetta italiana viene registrato nel 2011: una coppia formata da un maschio rilasciato nel 2006 e una femmina non inanellata, di cui non si conosce dunque la provenienza, che ha deposto tre uova e ha poi visto la nascita di due pulcini portati all'involo, dopo oltre quarant'anni dalla sua estinzione in Italia (Monti et al., 2014). Le nidificazioni hanno attualmente luogo sui nidi artificiali installati nei pressi di Bocca d'Ombrone. Nel periodo 2011-2018 si sono insediate complessivamente 4 coppie (1 nel PRM, 2 nella Riserva Naturale Diaccia Botrona ed 1 nell'Oasi WWF Laguna di Orbetello) le quali hanno portato all'involo di 33 pulcini, complessivamente (F. Monti, com. pers.) (Fig. 26). Nell'area del bacino del Mediterraneo e quindi anche nel Paese, durante le migrazioni e lo svernamento, vengono rilevati individui di origine Scandinava per quanto da tempo sia valutato importante l'apporto di individui di origine corsa (Melotti \& Spagnesi, 1979; Monti, 2012b). Nel passato la specie risulta indicata come migratrice regolare (Lovari \& Martelli, 1977). Risale all'inverno 1990-91 la prima segnalazione conosciuta per questa stagione: 1 ind. il 14 e 15/01/1991 alla foce del fiume Ombrone (Giovacchini, 1995f). Prime indicazioni di estivazioni, riconducibili ad immaturi: 1 ind. da giugno a settembre 1985 lungo il fiume Ombrone (GA e F. Favali in Arcamone \& Tellini, 1986); 1 ind. dal 20/05 al 26/06/1990 (Giovacchini, 1990b). Individuo inanellato: 1 ind. catturato il 15/12/1978 a Valle Maggiore, Grosseto, era stato inanellato il 11/07/1976 a Närpiö (Närpes), Finlandia (Bendini \& Spina, 1983).

\section{Accipitridae}

Falco pecchiaiolo Pernis apivorus $\mathrm{M}$ reg, B irr

Specie in decremento in Europa come nidificante (Staneva \& Burfield, 2017). La riproduzione riguarda zone boscate diversificate di latifoglie e conifere, pure o miste, anche di scarsa estensione, preferibilmente d'alto fusto, confinanti con aree erbose aperte ricche di imenotteri, con 


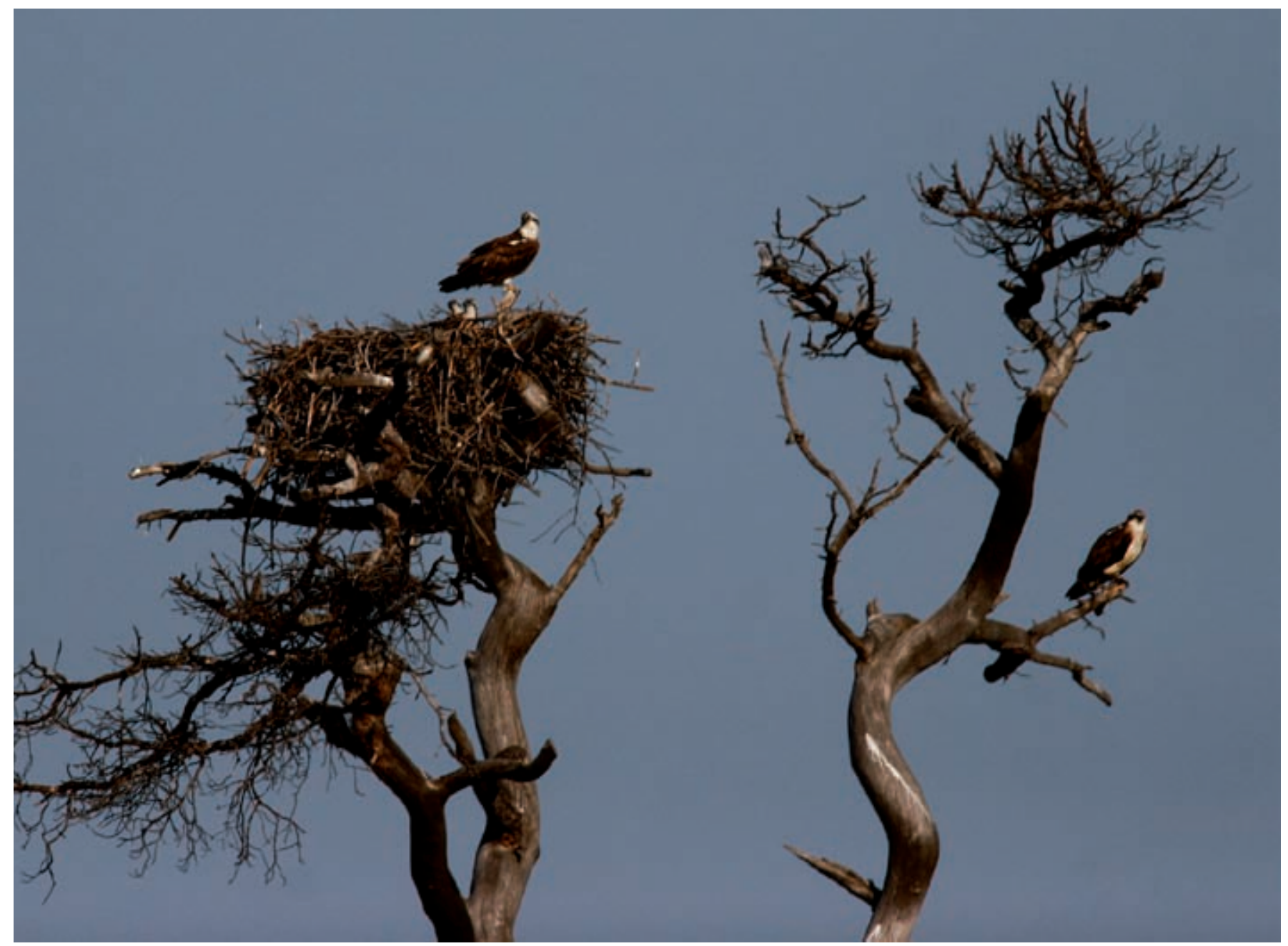

Fig. 26 - Falchi pescatori con giovani alla foce del fiume Ombrone. / Ospreys with young at Ombrone river mouth (Foto / Photo Giuseppe Anselmi).

maggiore diffusione tra 400-1000 m s.l.m. (Brichetti \& Fracasso, 2003). Migratore scarso nell'area protetta lungo una parte della fascia litoranea medio tirrenica. Limitata presenza anche in periodo riproduttivo, probabilmente per mancanza di habitat idoneo (Scoccianti \& Scoccianti, 1995). Nel PRM, dal 2001, sono stati rilevati indizi di nidificazione per i Monti dell'Uccellina, con osservazioni di adulti che decorrono dalla metà del mese di giugno: 1 ind. il 15/06/2001 a Collelungo (Fa. Perco, E. Arcamone e PG); 1 ind. il 30/06/2002 a Castel Marino (GA e D. Germani). Alla luce anche di queste indicazioni, la segnalazione di riproduzione certa di 1 coppia nella stagione 2006, ma non successivamente, assume quindi carattere episodico (Morimando et al., 2008; Petretti, 2008). Osservazione di presenza più tardiva registrata negli anni: 1 ind. il 22/10/1998 alla foce del fiume Ombrone (PG).

\section{Grifone Gyps fulvus A-2}

Accidentale. 1 ind. trovato morto inizio luglio 1992 in località Collecchio, ai margini del PRM (fonte: Quotidiano "La Nazione", edizione del 09/07/1992) (Giovacchini, 1999). Si deve ora aggiungere: 1 ind. in volo il 16/11/2015 nei pressi di Castel Marino (A. Gianni e P. Arrigucci).
Biancone Circaetus gallicus $\mathrm{M}$ reg, $\mathrm{B}$

Specie stabile in Europa come nidificante (Staneva \& Burfield, 2017). In Italia, è nidificante "Vulnerabile" (Peronace et al., 2012). Nidifica in boschi e boschetti di latifoglie sempreverdi, intervallati a vaste superfici nude, sabbiose o rocciose con parziale copertura erbacea e arbustiva, utilizzate per cacciare rettili, su versanti caldi e soleggiati, spesso scoscesi (Brichetti \& Fracasso, 2003). Le prime notizie circa la presenza della specie nel PRM giungono da Lovari \& Martelli (1977). La catena montuosa dell'Uccellina, caratterizzata prevalentemente da boschi termoxerofili, è luogo di riproduzione certa. In questo comprensorio i nidi vengono rinvenuti alla quota più bassa rilevata sino ad oggi nel Paese: $50 \mathrm{~m}$ s.l.m. (Petretti, 2008). Gli arrivi più precoci sin qui conosciuti si attestano entro la prima decade del mese di marzo, ad es. il giorno 08/03/1994 in località Salto del Cervo, Alberese (PG); il 02/03/1996 a San Rabano (GA); il 08/03/2001 sulla dorsale dei Monti dell'Uccellina, nei pressi di Talamone (PG). Successivamente la coppia provvede a costruire il sito riproduttivo, il quale può anche essere lo stesso della precedente stagione dove vengono osservate parate nuziali (Cattaneo \& Petretti, 1992). Ricerche condotte negli anni 


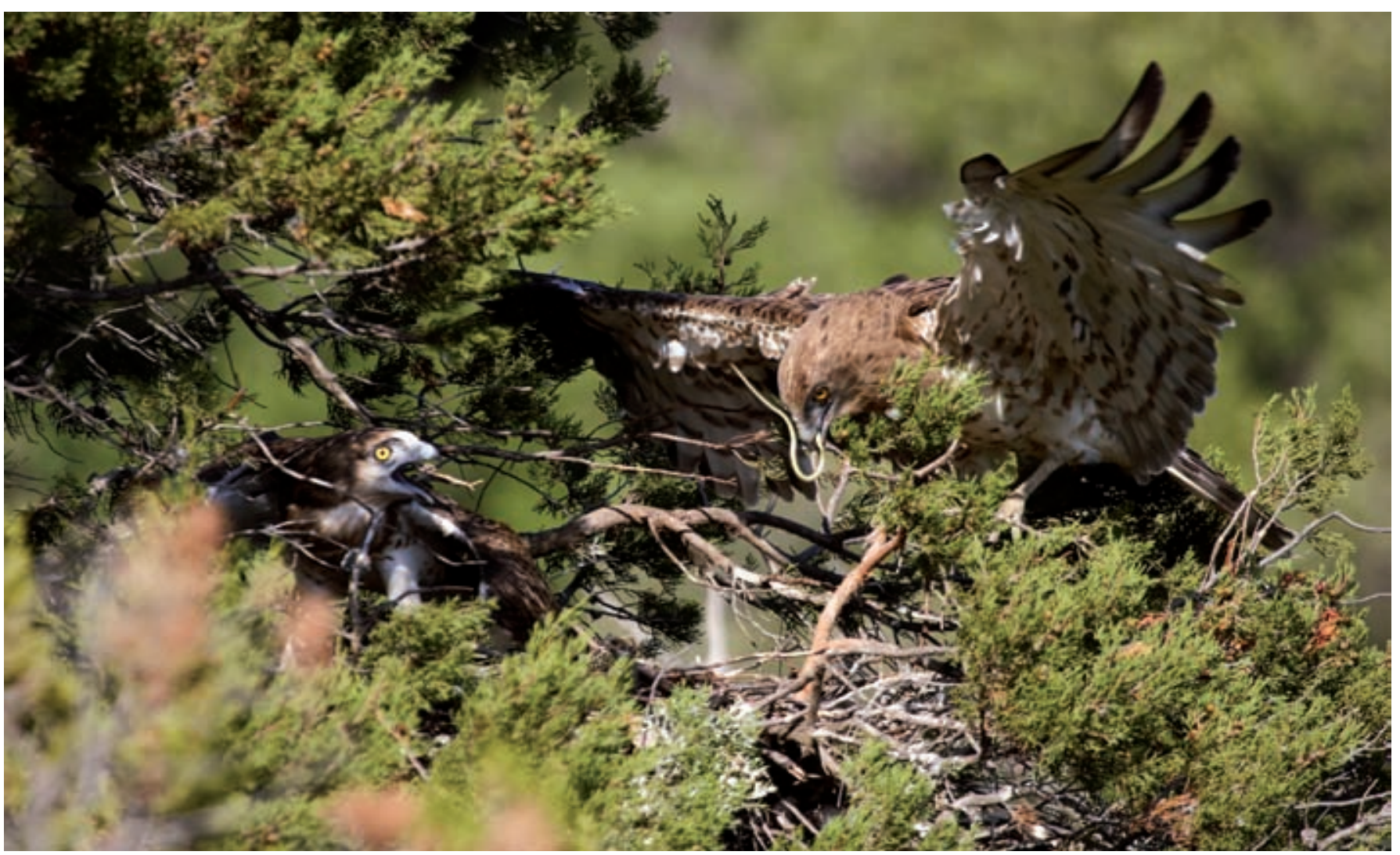

Fig. 27 - Bianconi al nido. / Short-toed Eagles at the nest (Foto / Photo Giuseppe Anselmi).

2006-2009 hanno definito la popolazione dell'area protetta che risulta compresa tra 4-6 coppie con una densità pari a 1 coppia $/ 30 \mathrm{~km}^{2}$ e con i territori di caccia più produttivi di quelli dei vicini Monti della Tolfa perchè più ricchi di erpetofauna (Petretti \& Rizzo Pinna, 2010) (Fig. 27). Occasionalmente osservate concentrazioni significative, ad esempio: sino a 6 individui a caccia nel maggio 2005 ai margini dei Monti dell'Uccellina, Talamone (GA). Segnalazione di presenza tardiva: 1 ind. in volo il 09/11/2012 nei pressi dello Scoglietto, Alberese (PG).

\section{Aquila anatraia minore Clanga pomarina A-1}

Accidentale. Specie precedentemente non rinvenuta (Giovacchini, 2006). Una segnalazione invernale: 1 ind. il 26/12/2006 alla foce del fiume Ombrone (M. Franchini, GA e E. Valentini in Arcamone \& Puglisi, 2008).

\section{Aquila anatraia maggiore Clanga clanga $\mathrm{M}$ irr, $\mathrm{W}$ irr}

In decremento in Europa come nidificante (Staneva \& Burfield, 2017). Nei periodi delle migrazioni e con lo svernamento frequenta zone umide costiere o interne con presenza marginale di aree boscate (Brichetti \& Fracasso, 2003). La presenza nel Paese pare contribuire, negli ultimi anni, all'ampliamento dei più vasti areali di svernamento della specie mentre, durante la migrazione autunnale, si può notare lo spostamento di individui verso la costa tirrenica per quanto la maggioranza della popolazione trascorra l'inverno più a sud (Zenatello et al., 2014). In questo quadro, la comparsa in Toscana sembra oggi più frequente rispetto ad un recente passato (Arcamone et al., 2007). Nel PRM, il quadro fenologico ricalca quanto rilevato a livello regionale, con un numero di segnalazioni decisamente incrementate rispetto allo scorso decennio (Giovacchini, 2006). Queste pervengono quasi unicamente dalla zona della Palude della Trappola e dalla foce del fiume Ombrone. Dall'inverno 1999/00 a quello 2018/19 sono infatti 10 le stagioni in cui ne è stata rilevata la presenza, unicamente con singoli individui (R. Nardi, F. Pratesi; GA; PG; F. Monti; LP; F. Pezzo, E. Savo, SL, C. Martelli, F. Bonucci, C. Castellani, E. Sbrana; G. Chiancianesi e C. Marti in EBN-Italia; fonte: COT, archivio PRM; CUA in Arcamone \& Puglisi, 2008; R. Maistri, L. Petruzzelli e P. Marotto su Ornitho. it). Le presenze paiono concentrarsi tra la fine di ottobre ed il mese di febbraio: in questo arco di tempo, durante la giornata, vengono quasi sempre utilizzati dal rapace, come punti di riposo e di osservazione, aree alberate prossime a spazi aperti della Tenuta della Trappola. Occasionali osservazioni fuori di tale periodo e zona: 1 ind. in volo il 25/04/2006 sulla strada di Collelungo, Alberese (LP).

Aquila minore Hieraaetus pennatus $\mathrm{M}$ irr?, W irr

Specie fluttuante in Europa come nidificante (Staneva $\&$ Burfield, 2017). Durante le migrazioni e lo svernamento è specie rilevata in aree pianeggianti o di collina, anche in vicinanza di aree boscate, comunque più facilmente ove si concentrano elevate presenze di uccelli (Brichetti \& Fracasso, 2003). Successivamente ad uno straordinario flusso migratorio verso i quartieri africani, che peraltro ha interessato buona parte del Paese, la stagione di svernamento 2004/05 ha visto ospitare nella provincia di Grosseto 2-3 
individui, di cui uno nel PRM (GA; Baghino et al., 2007). Nel 2013, nella stessa provincia, è stata resa nota la prima segnalazione di riproduzione in Italia (Petretti, 2013). La prima osservazione riferita all'area protetta è invece del precedente secolo: 1 ind. il 16/01/1988 alle Macchiozze (S. Benucci e P. Politi in Arcamone \& Tellini, 1991-1992). I dati radunati nell'area protetta fanno quasi sempre riferimento a singoli individui. Oltre alla citata stagione, gli inverni 2007/08 e 2015/16 sono risultati i più importanti per le frequenti osservazioni raccolte, da novembre a gennaio compresi (L. Rigacci; GA; PG; L. Bonanno su Ornitho.it, M. Cappelli su Ornitho.it). Migrazione primaverile verosimilmente irregolare, almeno lungo questa parte di costa, con osservazioni di individui apparentemente non oltre la prima decade di maggio: 1 ind. il 07/05/2007 (G. Serra in EBN-Italia).

\section{Aquila delle steppe Aquila nipalensis A-1}

Accidentale. 1 ind. il 25/11/2000 nella zona nord del PRM, al confine con Principina Mare (S. Benucci, P. M. Politi e L. Vanni).

\section{Sparviere Accipiter nisus SB, M reg, W reg}

Specie stabile in Europa come nidificante (Staneva \& Burfield, 2017). Nidifica in complessi forestali diversificati, collinari e montani, prediligendo quelli fitti di media grandezza, radurati e circondati da aree aperte, naturali o coltivate, utilizzate per cacciare, più diffusa tra 500-1600 $\mathrm{m}$ s.l.m.; durante le migrazioni e lo svernamento frequenta anche zone urbane dove può anche nidificare (Brichetti \& Fracasso, 2003). Nel PRM, una frazione della popolazione è molto probabilmente sedentaria. Specie elusiva, verosimilmente in incremento, è segnalata nei boschi di qualsiasi tipo sebbene le indicazioni raccolte in questi ultimi trent'anni evidenziano per l'area protetta una certa preferenza verso le fustaie miste e la pineta con sottobosco. Una conferma in tal senso giunge da Lovari \& Martelli (1977). Segnalato un caso di iperpredazione da parte di Falco peregrinus a carico della specie (Anselmi \& Corsi, 1992). In dettaglio, ai siti riproduttivi di Vergheria, Salto del Cervo e San Carlo, vanno aggiunti quelli della Valentina e delle Cannelle, nella zona di Talamone, ove la nidificazione è stata accertata rispettivamente almeno nelle stagioni riproduttive 2003 e 2008 (GA; PG). Nel periodo autunno-invernale, la maggiore concentrazione di uccelli in pianura favorisce l'incremento della popolazione. Infatti, da ottobre in poi, ne viene rilevata più facilmente la presenza in prossimità della foce del fiume Ombrone per quanto sia presumibile che già da ottobre, l'area possa ospitare svernanti provenienti dall'Europa settentrionale. Si segnala: 1 maschio il 12/12/2007 attaccato da 1 maschio di Falco columbarius, Alberese (PG). Nel periodo 2000-2004 stimata la presenza di 0,48 coppie/10 km2 (Giovacchini, 2006). Stimabile una presenza minima di 5-6 coppie nidificanti. Un individuo albino catturato a Talamone il 12 dicembre 1918 (Arrigoni degli Oddi, 1929).

\section{Astore Accipiter gentilis $\mathrm{M}$ reg ? W irr}

Specie in decremento in Europa come nidificante (Staneva \& Burfield, 2017). Consistenza e fenologia dei movimenti poco conosciuti; fenomeni di erratismo limitati ai soggetti immaturi (Benussi, 1992). Nel passato considerata unicamente specie a comparsa irregolare nel PRM (Martelli, 1983). A tutti gli effetti, occorre attendere la metà circa degli anni ' 90 del secolo scorso per riosservare il rapace, forse anche perchè in precedenza confuso con Accipiter nisus: 1 ind. in volo il 30/12/1994 sui Monti dell'Uccellina (Fabbrizzi, 1995a). Esclusa la nidificazione nell'area protetta (Cauli \& Genero, 2017). Si segnala: 1 ind. il 08/12/2006 alla foce del fiume Ombrone (F. Piccolo in EBN-Italia); 1 ind. impegnato ad emettere richiami nell'aprile 2009 in località Cannelle, Talamone (GA); 1 ind. il 14/02/2010 a Castel Marino, Alberese (F. Fabbrizzi in GOM-List); 1 femmina il 24/10/2010 in località Le Grotte, Alberese (C. Martelli e A. Martelli in GOM-List).

\section{Falco di palude Circus aeruginosus $\mathrm{M}$ reg, W reg}

Specie in incremento in Europa come nidificante (Staneva \& Burfield, 2017). Durante le migrazioni e lo svernamento frequenta, oltre alle zone palustri, anche aree coltivate, fiumi, canali e pascoli (Brichetti \& Fracasso, 2003). Negli anni che fanno immediatamente seguito all'istituzione dell'area protetta la nidificazione è stata ritenuta probabile nei chiari della Palude della Trappola (Lovari \& Martelli, 1977). Nel successivo decennio, in presenza di un quadro fisionomico della vegetazione ad elofite mutato per via del ripetuto ingresso di acqua salata dal mare a causa dell'erosione costiera, la riproduzione risultava già assente (Liberatori et al., 1988; Corsi \& Anselmi, 1991; GA; PG). Nella stagione invernale ed nella fase post-riproduttiva il rapace appare meno selettivo rispetto all'habitat. Negli anni 2013-2017 le osservazioni sullo svernamento nel PRM si riferiscono ad un massimo di 8 individui censiti il 18/01/2015 nelle zone umide della Trappola, foce del fiume Ombrone e Macchiozze (fonte: COT, archivio PRM). Adulti e giovani gravitanti nell'area si riuniscono, per trascorrere la notte insieme ad altri accipitridi, nel dormitorio posto nella Riserva Regionale Diaccia-Botrona (Arcamone et al., 2007). In inverno è possibile rilevare la presenza di un dormitorio utilizzato irregolarmente dalla specie nel Chiaro del Porciatti, all'interno della Palude della Trappola, che viene anche condiviso con un'altra specie di accipitride, ad es. 3 immaturi il 08/12/2015 insieme a 3 maschi di Circus cyaneus (L. Rigacci). Durante la stagione invernale la specie viene contattata più raramente nel settore meridionale di Talamone, mentre in periodo riproduttivo risulta generalmente circoscritta a pochi esemplari erratici.
Albanella reale Circus cyaneus $\mathrm{M}$ reg, $\mathrm{W}$ reg
Specie in decremento in Europa come nidificante (Sta- neva \& Burfield, 2017). Con le migrazioni e lo svernamen- to viene rinvenuta in ambienti aperti, generalmente erbosi sia di pianura, che montani (Brichetti \& Fracasso, 2003). Osservata con singoli individui nel settore settentrionale e centrale del PRM, da Principina Mare al Collecchio, in perlustrazione negli spazi aperti pianeggianti, caratterizza- ti da incolti, prati pascolo e zone umide. In misura minore, il rapace viene anche rinvenuto a caccia nei rilievi colli- nari e nel settore meridionale dell'area protetta. Adulti e giovani gravitanti nell'area si riuniscono, per trascorrere la notte insieme ad altri accipitridi, nel dormitorio posto 
nella Riserva Regionale Diaccia-Botrona (Arcamone et al., 2007). In inverno, tuttavia, è possibile rilevarne un altro nel Chiaro del Porciatti, all'interno della Palude della Trappola, utilizzato irregolarmente dalla specie, anche con altro accipitride, ad es. 3 maschi il 08/12/2015 insieme a 3 immaturi di Circus aeruginosus (L. Rigacci; cfr. specie precedente). Tale comportamento viene osservato già nello scorso secolo, unicamente con Circus cyaneus, ad es. sino a 6 ind. il 28/11/1999 (GA). L'utilizzazione dello stesso Chiaro del Porciatti per identiche finalità è confermata ancora nell'inverno 2017/18, ma con numeri estremamente bassi: sino a 2 ind. il 30/12/2017 (PG e A. Picciau). La popolazione svernante, gravitante nell'intero comprensorio dell'area protetta, è valutata ogni anno in 1-2 esemplari (Fabbrizzi, 1995b). Nella fase conclusiva della sua permanenza invernale può condividere con Circus pygargus gli ambienti nei quali si dedica alla ricerca del cibo.

\section{Albanella pallida Circus macrourus M irr}

In Europa, è nidificante con trend sconosciuto (Staneva \& Burfield, 2017). Con le migrazioni si rileva in ambienti aperti costieri e di pianura come: pascoli, coltivi e margini di zone umide (Brichetti \& Fracasso, 2003). 1 maschio il 01/04/1997 nei pressi della località Pianacce, Alberese (Giovacchini, 1999). Si aggiungono ora i seguenti dati riferiti unicamente a maschi: 1 ind. il 04/02/2005 nei pressi di Vaccareccia, Alberese (F. Fabbrizzi); 1 ind. il 07/02/2005 alla foce del fiume Ombrone (GA); 1 ind il 12/10/2014 a Spergolaia (M. Dragonetti in GOM-List).

\section{Albanella minore Circus pygargus $\mathrm{M}$ reg}

In Europa, è specie nidificante con trend sconosciuto (Staneva \& Burfield, 2017). Frequenta una grande varietà di spazi aperti, sia naturali che coltivati, e con rada copertura arborea od arbustiva; moderatamente gregario durante le migrazioni (Brichetti \& Fracasso, 2003). La nidificazione del rapace all'interno del perimetro del PRM non è stata ancora rilevata per quanto i siti più vicini distino circa $1,2 \mathrm{~km}$ in linea d'aria (F. Farsi e Progetto WWFProvincia di Grosseto "Albanella minore"; GA; PG; Corsi \& Anselmi, 1991). La specie viene rinvenuta dai primi giorni di aprile sino a settembre: nella fase iniziale del suo arrivo può condividere parte degli ambienti con Circus cyaneus, prossima alla partenza. Le indicazioni raccolte nell'area protetta riguardano quasi esclusivamente singoli individui di sesso maschile. Rilevata nei distretti settentrionali e centrali della Trappola, di golena del fiume Ombrone sino a Campo al Pino, Spergolaia e, meno frequentemente, in quello di Alberese Scalo-Collecchio sino alla Bonifica di Talamone, ai limiti meridionali del PRM. Osservazioni più recenti: 1 maschio il 10/05/2015 in località Vaccareccia (PG); 1 femmina il 07/06/2015 nei pressi del Podere Isonzo, Alberese (PG); 1 maschio il 03/06/2017 nei pressi delle golene di Alberese lungo il fiume Ombrone (GA). Rare le segnalazioni che giungono dalla Palude della Trappola. Nella fase post-riproduttiva adulti e giovani gravitanti anche nell'area protetta si riuniscono per trascorrere la notte insieme ad altri accipitridi verosimilmente nel roost posto nella Riserva Regionale Diaccia-Botrona. Individuo inanellato: 1 ind. catturato in
Alberese il 10/09/1950, era stato inanellato da pullus il 30/05/1950 nei pressi di Amburgo, Germania (Bendini \& Spina, 1983).

Nibbio reale Milvus milvus $\mathrm{M}$ reg, $\mathrm{W}$ irr

Specie in decremento in Europa come nidificante (Staneva \& Burfield, 2017). Durante le migrazioni frequenta anche campagne coltivate, risaie, laghi, lagune e pascoli; nel periodo dello svernamento può formare dormitori di decine di individui (Brichetti \& Fracasso, 2003). Le prime indagini sull'avifauna del PRM giungono a segnalarne la presenza (Lovari \& Martelli, 1977). Già nei primi anni '90 del secolo scorso il rapace compare verosimilmente con maggiore frequenza, quasi sempre con singoli individui in migrazione, in ambienti aperti di pianura come la foce del fiume Ombrone, San Mamiliano, Spolverino, sino alle propaggini meridionali del promontorio di Talamone, anche nei pressi delle falesie dell'Uccellina (Giovacchini, 2006). Un ulteriore incremento delle osservazioni è da attribuirsi anche ai risultati raccolti sin dall'anno 2011 nell'ambito di importanti progetti LIFE-Natura (Ceccolini et al., 2013). Tra le più recenti osservazioni: 1 ind. il 17/04/2013 in località Magazzini, Alberese (R. Ragazzini); 1 ind. il 12/03/2015 lungo il canale di Collelungo (C. Martelli in GOM-List). Presenze invernali apparentemente irregolari: inizialmente negli anni 1985-1989 con un massimo di 2 individui nella Palude della Trappola e zone adiacenti (Corsi \& Anselmi, 1991); 1 ind. il 11/12/1994 a Spolverino (A. Chimenti); più recentemente, recuperate informazioni di individui con data logger nelle stagioni 2012/13, 2013/14 e 2014/15 nell'ambito del progetto LIFE-Natura "Save the Flyers" (G. Ceccolini e A. Cenerini, com. pers.).

\section{Nibbio bruno Milvus migrans $\mathrm{M}$ reg}

In Europa, è specie nidificante con trend incerto (Staneva \& Burfield, 2017). Nei periodi delle migrazioni frequenta una ampia varietà di ambienti costieri e montuosi (Brichetti \& Fracasso, 2003). Nel PRM, segnalato inizialmente da Lovari \& Martelli (1977). Le osservazioni riguardano quasi sempre singoli individui nelle aree pianeggianti, anche interne, dell'area protetta. Presenza occasionale a novembre con individui attardatisi durante gli spostamenti: 1 ind. il 14/11/1995 alle Macchiozze (PG). La migrazione primaverile pare essere meno diluita rispetto a quella autunnale. Rare osservazioni in periodo estivo: 2 ind. il 03/06/1999 in località Lasco, Alberese (GA). Rinvenuto anche in alimentazione su carcasse di ungulati: 1 ind. il 18/04/2003 in località Rimessini, Alberese (GA). Tra le più recenti osservazioni: 1 ind. il 29/03/2016 in località Apparita, Talamone (GA); 1 ind. il 07/09/2016 in località San Mamiliano, Trappola (PG).

\section{Aquila di mare Haliaeetus albicilla $\mathrm{M}$ irr, W irr}

In Europa, è specie nidificante in incremento (Staneva \& Burfield, 2017). Durante le migrazioni e con lo svernamento frequenta zone paludose di acqua dolce, lagune, valli salmastre, laghi ed aree coltivate alberate (Brichetti \& Fracasso, 2003). Nel PRM, rilevata esclusivamente nelle zone umide della Trappola, alla foce del fiume Ombrone ed in aree immediatamente circostanti. Osservazio- 
ni all'inizio del corrente secolo: 1 ind. il 12/01/2001 nei pressi della foce del fiume Ombrone (GA). Successivamente, con regolarità, sino al 19/04/2001, tra la Palude della Trappola, la Tenuta di San Carlo e la foce del fiume Ombrone (GA; G. Manganelli e G. Cappelli; PG e G. F. Martini; A. Gianni). Segnalazione invernale anche nel successivo anno: 1 ind. il 27 e 28/01/2002 alla foce del fiume Ombrone (G. Sammuri e R. Nardi). Brogi (1896) riferisce "Un individuo femmina, di un gruppo di quattro, catturato il 6 aprile 1896 nella Tenuta "La Trappola", erano intenti a mangiare gli intestini di agnelli uccisi il giorno prima".

Poiana calzata Buteo lagopus A-2

Accidentale. 1 ind. il 18/11/1995 in località Pratini, Alberese (Giovacchini, 1999). Si deve ora aggiungere: 1 ind. il 22/09/2005 in volo sul Collecchio (PG).

Poiana Buteo buteo SB, M reg, W reg

In Europa, è nidificante stabile (Staneva \& Burfield, 2017). Nei periodi delle migrazioni e durante lo svernamento frequenta preferibilmente aree planiziali e costiere (Brichetti \& Fracasso, 2003). Nonostante la natura eclettica nella scelta del sito riproduttivo, nel PRM mostra maggiore preferenza verso i boschi misti di latifoglie governati a fustaia, specialmente se con piante adeguatamente coperte da Hedera helix. I dati raccolti in quasi venti anni (1986-2004) portano a stimare una bassa densità minima delle coppie nidificanti $\left(0,6\right.$ coppie $\left./ 10 \mathrm{~km}^{2}\right)$, conseguenza anche della generale rarefazione degli habitat idonei nel settore costiero tirrenico (Giovacchini, 2006; Fornasari et al., 2010). Più recentemente indicate 6-7 coppie (Petretti, 2008). Durante la riproduzione, la specie è segnalata nella zona della Tenuta di Torre Trappola, in prossimità del confine con la Tenuta di San Carlo, ed ancora, con indicazioni di nidificazione certa nelle vicinanze della strada del Condotto di Alberese e nel comprensorio tra Torre Bassa e Torre della Bella Marsilia, Collecchio, mentre è probabile nella zona della Valentina, Talamone (GA; S. Celletti; PG; F. Fabbrizzi). Stimabile una presenza minima di 3-4 coppie nidificanti. In inverno incremento evidente dei contingenti come in buona parte della Toscana (Savio, 1997). Rare le osservazioni di individui gregari in migrazione, ad es. 4 ind. il 07/04/1996 in località Vaccareccia (PG).

\section{GRUIFORMES}

\section{Rallidae}

Porciglione Rallus aquaticus $\mathrm{M}$ reg, B ?, W reg

In Europa, è specie nidificante con trend incerto (Staneva \& Burfield, 2017). Nidifica in zone umide di acqua dolce, anche di ridotte dimensioni e con fondali poco profondi, localmente in stagni salmastri, comunque con maggiore diffusione sino a $500 \mathrm{~m}$ s.l.m.; durante lo svernamento frequenta preferibilmente zone umide costiere, anche salmastre (Brichetti \& Fracasso, 2004). Verosimilmente sottostimato a causa della sua indole particolarmente elusiva. Nel PRM, specialmente durante l'autun- no-inverno, possibili contatti con individui in richiamo ai margini di ambienti umidi della fascia costiera purché in tratti con scarsa profondità e sponde bordate da vegetazione erbacea palustre, anche di superficie limitata, tra $\mathrm{i}$ quali: foce del fiume Ombrone, Chiari del San Carlo e Palude della Trappola, Fosso Razzo e Canale Essiccatore. In periodo riproduttivo rinvenuto occasionalmente nei canali adiacenti alle Macchiozze e al confine tra le tenute della Torre Trappola e di San Carlo, rispettivamente: 1 ind. il 12/05/2000 (PG); 1 ind. il 04/05/2007 (PG). Durante le migrazioni segnalato occasionalmente in contesti inusuali: 1 ind. il 12/10/1986 nel laghetto del Precoriale, Alberese (PG); 1 ind. il 11/03/1991 al Paduletto di Collelungo (PG). Dato storico: 1 ind. il 13/07/1969 nella Palude della Trappola (Castellani, 1970).

Re di quaglie Crex crex A-1

Accidentale. Specie precedentemente non rinvenuta (Giovacchini, 2006). 1 ind. il 09/05/2008 alla foce del fiume Ombrone (GA).

\section{Schiribilla Porzana parva M irr?}

In Europa, è nidificante stabile (Staneva \& Burfield, 2017). Di indole schiva, per quanto risulti piuttosto tollerante alla presenza umana, durante le migrazioni frequenta zone umide di acqua dolce con ricchi ammassi sparsi di vegetazione secca in spazi aperti (Brichetti \& Fracasso, 2004). Nel PRM, presenze difficilmente valutabili, verosimilmente scarse. Si ritiene di sospendere una segnalazione invernale già nota (M. Pani, ined.): 1 ind. il 13/01/1984 nella Palude della Trappola (Giovacchini, 2006)

\section{Schiribilla grigiata Porzana pusilla A-1}

Accidentale. 1 ind. trovato morto il 09/04/1989 alla foce del fiume Ombrone (Giovacchini, 1999).

\section{Voltolino Porzana porzana M irr? W irr}

In Europa, è specie nidificante con trend sconosciuto (Staneva \& Burfield, 2017). Generalmente schivo, durante le migrazioni e lo svernamento frequenta ambienti palustri con vegetazione erbacea piuttosto folta, ma non troppo alta alternati a spazi aperti ove si possono rinvenire più spesso singoli individui (Brichetti \& Fracasso, 2004). Nel PRM, le osservazioni hanno inizio dagli anni '90 del secolo scorso, in concomitanza con il miglioramento della copertura dell'area da parte di esperti rilevatori. In migrazione: 1 ind. il 17/09/1990 alla foce del fiume Ombrone (PG); 1 ind. dal 9 al 23/11/2012 alle Macchiozze ed in prossimità del contiguo Canale Essiccatore (PG). Con riferimento al periodo invernale: 1 ind. il 01/12/2000 nei pressi della foce del fiume Ombrone (PG); 1 ind. il 11/01/2003 nella Palude della Trappola (CUA in Arcamone \& Puglisi, 2006).

\section{Gallinella d'acqua Gallinula chloropus SB, M reg, W reg}

In Europa, è nidificante stabile (Staneva \& Burfield, 2017). Nidifica in zone umide di varia natura, anche di ridottissime dimensioni, con vegetazione palustre diversificata e acque ferme o debolmente correnti, preferibilmente dolci, generalmente sino a $500 \mathrm{~m}$ s.l.m.; durante le 
migrazioni e lo svernamento frequenta per motivi trofici anche marcite, prati asciutti e coltivi distanti dalle zone umide (Brichetti \& Fracasso, 2004). Nel PRM, viene rilevata più facilmente, per esempio, lungo il fiume Ombrone, nel Canale Essiccatore, Padule della Trappola e Chiari del San Carlo, Canale di Bonifica di Talalmone, Canale Collelungo, Fosso Razzo, laghetti ad uso irriguo. Rilevato occasionalmente nel Paduletto di Collelungo (PG). Data l'eterogeneità dei siti utilizzati nonchè il suo comportamento elusivo, i contingenti della specie risultano quindi di difficile stima. Quello nidificante nell'area protetta può essere tentativamente stimato in almeno 25-30 coppie (Fig. 28).

\section{Folaga Fulica atra SB, M reg, W reg}

In Europa, è specie nidificante in decremento (Staneva \& Burfield, 2017). Per la nidificazione la specie frequenta ambienti palustri o di acqua dolce, anche debolmente correnti, generalmente sino a 400-500 m s.l.m., caratterizzati più comunemente dalla presenza di una cintura rivierasca e di letti di vegetazione palustre emergente ove vengono rilevate coppie sparse; in periodo non riproduttivo è rinvenuta anche in corpi idrici con sponde prive di effettiva copertura di vegetazione emergente, ma con idrofite sommerse (Brichetti \& Fracasso, 2004). In tutte queste situazioni si distingue tra i rallidi per essere la specie più confidente nei confronti dell'uomo. La Ma- crozona "Maremma Grossetana" è un sito di importanza regionale per lo svernamento della specie (Arcamone et al., 2007). Nei primi anni dall'istituzione dell'area protetta rinvenuta anche con numeri oggi difficilmente raggiungibili: 600 ind. il 08/02/1976 nella Palude della Trappola (Chelini, 1983). Nel PRM, nel periodo 2013-2017, vengono mediamente censiti 149 individui per stagione di svernamento (fonte: COT, archivio PRM). Verosimilmente, una frazione piuttosto limitata della popolazione gravitante nell'area protetta potrebbe essere sedentaria. Nidificazioni circoscritte nelle zone delle Macchiozze e del Canale Essiccatore, sebbene se ne rilevi irregolarmente la presenza in piccoli bacini artificiali ad uso irriguo del Collecchio così come lungo il Canale di Bonifica di Talamone ed alla Voltina. In quest'ultima località, la riproduzione è regolare (M. Colli; F. Corsi; GA; PG; F. Farsi; S. Paoloni). Da un esame dell'andamento delle riproduzioni nel corso di più anni, sembra sussistere una correlazione della nidificazione con l'entità delle precipitazioni atmosferiche in primavera ed inizio estate. Piogge abbondanti garantiscono il raggiungimento di livelli idrometrici idonei alla nidificazione nei corpi d'acqua isolati, come per esempio succede alle Macchiozze: 1 ad. con 3 pulli il 10/06/1996 (PG); 2 pulli il 12/06/2003 (GA e PG). Concentrazioni estive: ca. 150 ind. adulti il 29/06/2015 nella Palude della Trappola (PG). Stimabile una presenza minima di 1-2 coppie nidificanti.

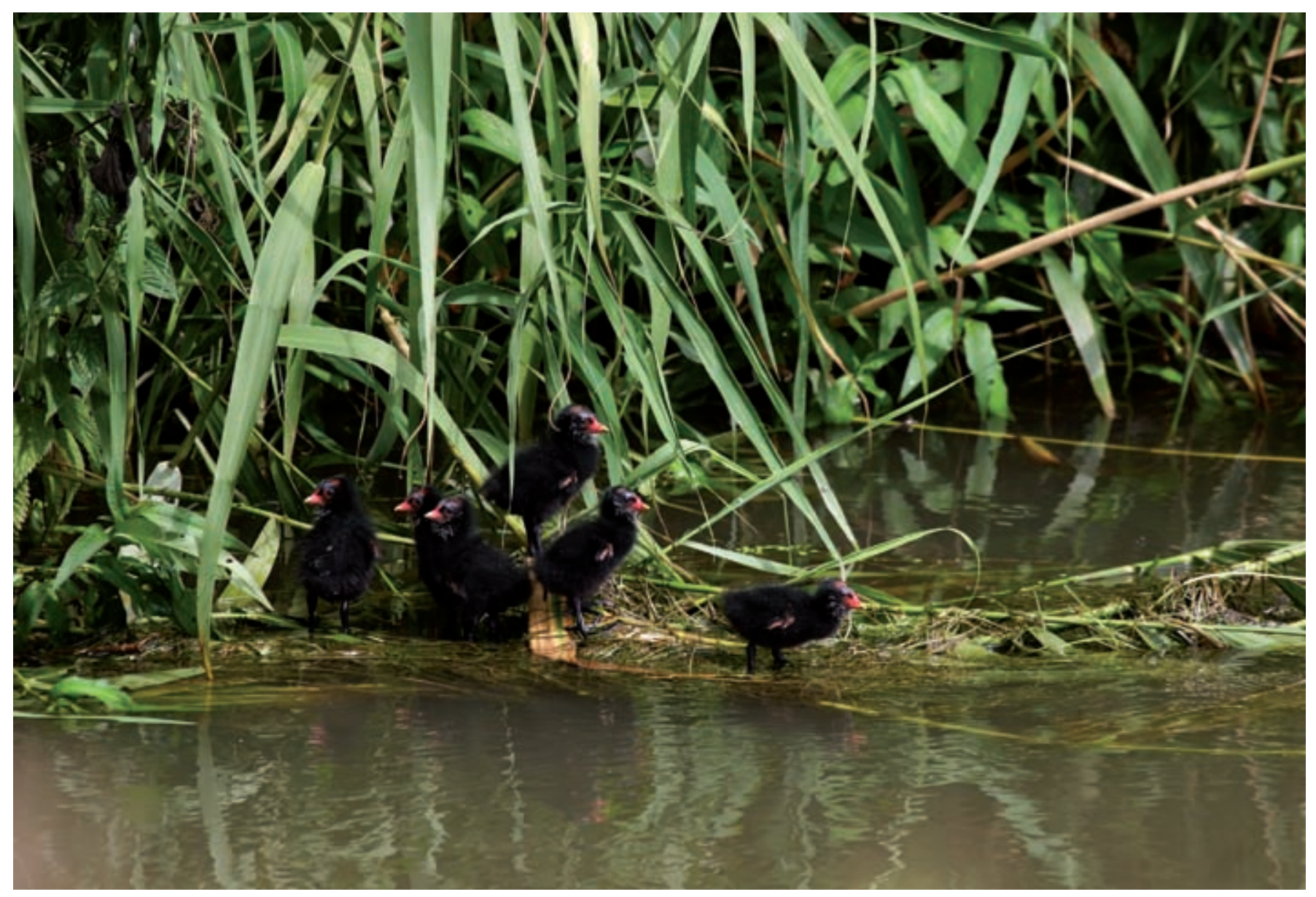

Fig. 28 - Pulcini di gallinella d'acqua. / Moorhen chicks (Foto / Photo Giuseppe Anselmi). 


\section{Gruidae}

Gru Grus grus $\mathrm{M}$ reg, W reg

Specie in incremento in Europa come nidificante (Staneva \& Burfield, 2017; Baccetti et al., 2017). In inverno e durante le migrazioni risulta decisamente gregaria e legata maggiormente a spazi aperti erbosi, zone umide ed aree coltivate anche estensivamente (Brichetti \& Fracasso, 2004). È della stagione 1991/92 il primo caso di svernamento registrato nel PRM, con 11 individui dal 21/12/1991 al 14/02/1992 (Giovacchini 1995g; Arcamone et al., 2007). In quello stesso periodo vengono rilevati occasionali spostamenti per finalità trofiche verso la sola Diaccia Botrona, comportamento che si è poi regolarizzato negli anni (F. Corsi, com. pers.). Attualmente, molti individui utilizzano in maniera complementare comprensori limitrofi come le Risaie di Principina o aree più interne della pianura, tuttavia recandosi regolarmente nell'area protetta per il pernottamento. La Macrozona "Maremma Grossetana", in ragione di un più deciso incremento dei contingenti e della presenza di un roost nella Palude della Trappola, ha assunto (in particolare negli ultimi cinqueotto anni) un ruolo prioritario per lo svernamento in ambito regionale, per quanto sia da rilevare che nel passato venissero utilizzati per le medesime finalità anche altri comprensori interni o limitrofi all'area protetta, ad es. 7-9 ind. almeno dal 09/01 al 23/01/2001 alle Macchiozze (PG e GA); 209 ind. il 20/02/2011 in località Voltina
(GA). Nel periodo 2013-2017 sono stati censiti 9-426 individui svernanti (fonte: COT, archivio PRM). Presenze invernali massime raggiunte con i conteggi serali al rientro nel dormitorio della Palude della Trappola: 600 ind. il 08/01/2017 (PG e A. Picciau); 610 ind. il 30/12/2017 (PG e A. Picciau). La specie viene osservata con maggiore regolarità dal 1997, mentre dal 2008 si registra un tendenziale anticipo negli arrivi dei contingenti nell'area protetta, con i primi individui segnalati tra la seconda quindicina di ottobre e la successiva di novembre (Fig. 29). Tale andamento potrebbe dipendere, nei comportamenti, da un rafforzamento di strategie migratorie alternative che vedono anche una maggiore incidenza numerica degli svernanti lungo la costa tirrenica (Mingozzi et al., 2013; Zenatello et al., 2014). Sin dal 2011 la lettura di anelli in PVC di più individui $(n=3)$ mostra la rilevanza dell'area per i contingenti finlandesi, registrando nel contempo un caso di fedeltà al sito nell'inverno 2017/18 (F. Corsi; PG; A. Picciau; SL; F. Monti) (fonte: archivi Ringing Centre Finnish Museum of Natural History - LUOMUS; ISPRA). Le cifre rilevate nei due momenti estremi di occupazione del PRM presentano al 2017 una tendenza ad esaurire le partenze nella seconda quindicina di febbraio. Le medie calcolate per gli anni 2008-2017 indicano 13,7 individui per il periodo iniziale di osservazioni (i primi 10 giorni di presenza) e 134,4 individui per quello finale (gli ultimi 10 giorni di presenza) (PG). Conferme in tal senso, tra le più recenti: almeno 500 ind. il 21/02/2016 in

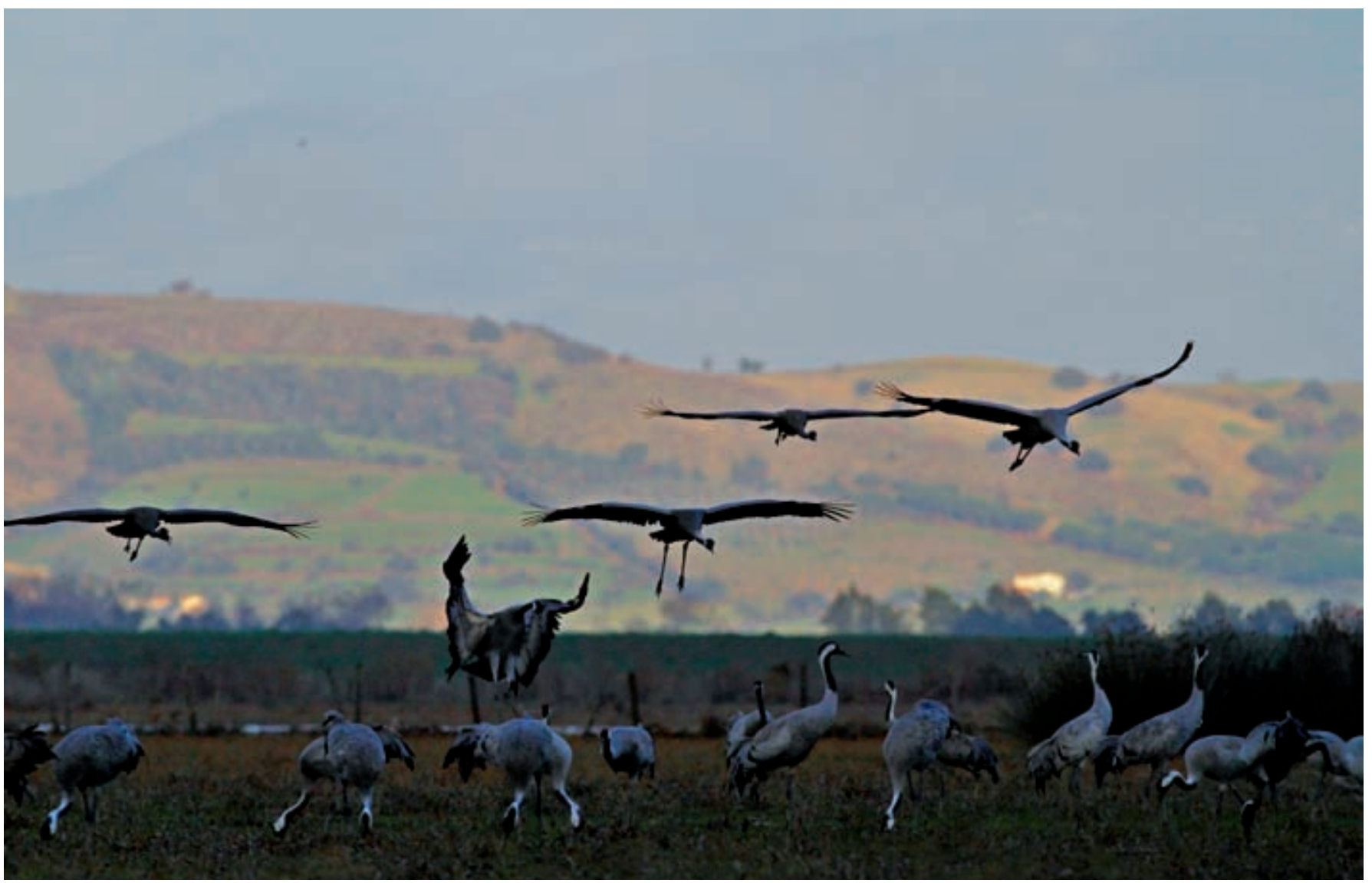

Fig. 29 - Gru alle Macchiozze. / Cranes at the Macchiozze (Foto / Photo Giuseppe Anselmi). 
volo sulla Palude della Trappola (LP in GOM-List); 259 ind. il 18/02/2017 complessivamente tra le Macchiozze, Spergolaia e Spolverino (A. Galimberti e G. Rossi su Ornitho.it). Al contrario, per quanto anch'esse provvedano analoghe disponibilità di risorse trofiche, le aree collocate nella fascia più interna della provincia, forse perché meno tutelate dal disturbo antropico, forniscono un quadro della presenza della specie che si discosta da quello osservato nell'area protetta (Corsi \& Dragonetti, 2010; Corsi \& Colli, 2013). Presenze estive: 1 ind. il 27/08/2007 in volo sulla Bonifica di Talamone (A. Chimenti); 12 ind. il 9 e 10/08/2011 in volo nei pressi della foce del fiume Ombrone ed alla Trappola (M. Colli e C. Martelli in GOM-List). Osservato un raro caso di individuo leucistico il 30/11 e 01/12/2014 in località Spolverino, Alberese (Colli \& Giovacchini, 2016). Distribuzione e consistenza massima dei contingenti in periodo autunno-invernale raffigurati in Fig. 30.

\section{CHARADRIIFORMES}

\section{Burhinidae}

Occhione Burhinus oedicnemus M reg, B, W reg

Specie in incremento in Europa come nidificante (Staneva \& Burfield, 2017). In Italia, è nidificante "Vulnerabile" (Peronace et al., 2012). Durante tutto l'anno frequenta spazi aperti su substrati aridi e con copertura erbacea-arbustiva rada, mostrando un crescente adattamento alle situazioni offerte da svariate attività umane; durante

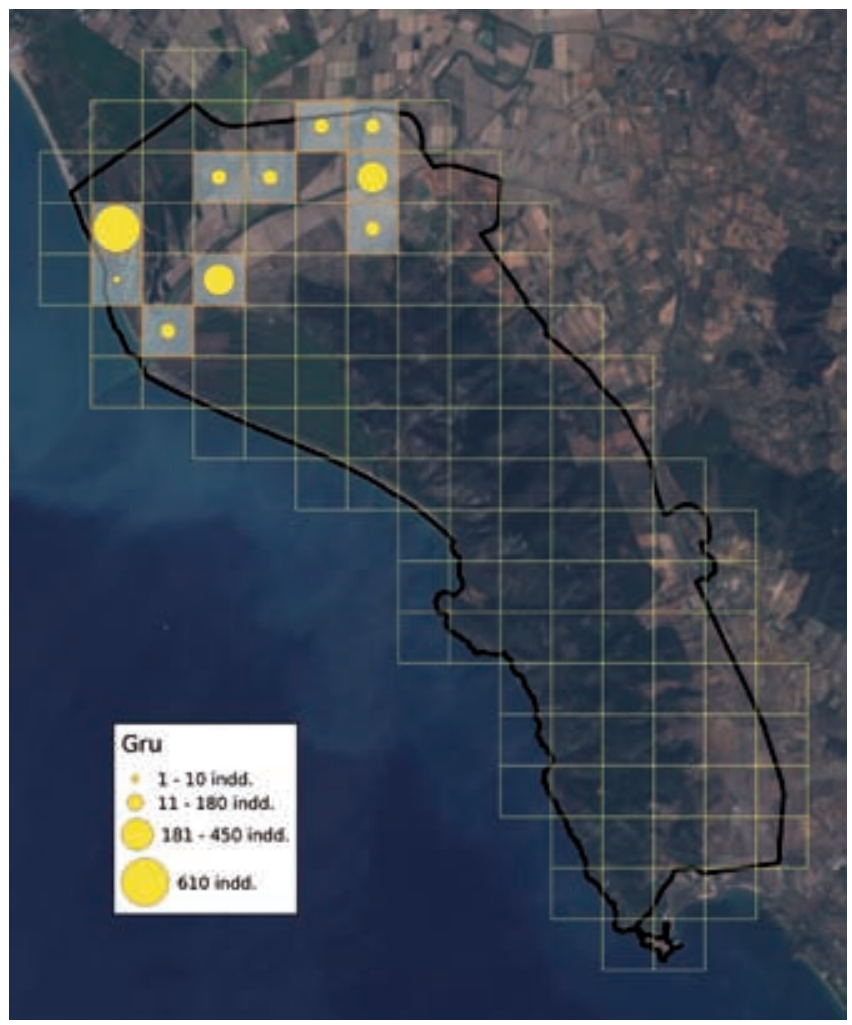

Fig. 30 - Siti di presenza della gru in periodo autunno-invernale. / Sites of presence of Crane in autumn-winter season (1991-2017). la nidificazione è rilevato generalmente nelle steppe cerealicole, nei pascoli, ai margini di zone umide, nei prati pascoli e nei ghiareti, normalmente sino a $300 \mathrm{~m}$ s.l.m., così come nei periodi delle migrazioni e dello svernamento quando, gregario, frequenta sostanzialmente gli stessi ambienti costieri e interni (Brichetti \& Fracasso, 2004; Tinarelli et al., 2009). Criptico e crepuscolare e perciò verosimilmente sottostimato, in primavera ed estate può essere contattato più facilmente attraverso gli individui in canto spontaneo al crepuscolo, per quanto questa attività prosegua durante tutto l'anno $\mathrm{e}$, in misura ridotta, anche nel periodo invernale (Giovacchini et al., 2012). Per quanto annoverabile tra gli uccelli "acquatici" la specie si distingue per essere rilevata in una più ampia disponibilità di habitat congeniali, anche su base locale (Giovacchini \& Stefanini, 2008; Picciau, 2017). Nel PRM, sono frequentati alcuni ambienti tra i più tipici tra quelli in cui viene rinvenuto in Italia, quali prati pascoli, steppe alofile ed incolti con bassa vegetazione, nelle zone comprese tra Spergolaia, Macchiozze, foce del fiume Ombrone, Trappola e Tenuta di San Carlo (Meschini \& Fraschetti, 1989; GA e PG). Compare raramente in ambiente dunale: 1 ind. il 23/11/2009 alla Trappola (PG). Una frazione della popolazione potrebbe essere costituita da individui sedentari. Attraverso la metodologia del conteggio degli individui in canto in periodo riproduttivo, negli anni 1998-2001 è stata valutata una popolazione complessiva di 6-8 coppie sparse, incrementata a 9-10 per la stagione 2005 (PG). Le presenze invernali registrate nei decenni succssivi alla scoperta avvenuta nel 1986 di un importante area di raduno diurno collettivo, confermano la rilevanza del dormitorio stagionale posto nei pascoli della Palude della Trappola, per quanto non utilizzato regolarmente e in modo complementare ad altri esterni al PRM (GA in Arcamone \& Tellini, 1987; Dragonetti et al., 2014) (Fig. 31). Alcuni tra i più recenti conteggi effettuati al dormitorio invernale nella Palude della Trappola: 12 ind. il 16/01/2004 (PG); 13 ind. il 14/01/2017 (A. Picciau, PG e M. Dragonetti); 10 ind. il 30/12/2017 (PG, A. Picciau, F. Ferragamo Visconti e G. Visconti). Segnalazioni apparentemente irregolari in altre località: 32 ind. il 16/01/2018 ai Rimessini di Alberese (GA); 7 ind. il 08/12/2018 alla foce del fiume Ombrone (PG, A. Canci, C. Martelli, F. Carpita, S. Parenti). In questo periodo dell'anno, i singoli individui contattati a più riprese indicano in ogni caso una regolarità nella frequentazione delle aree di alimentazione, specialmente di quelle della Trappola. Stimabile una popolazione minima di 7-8 coppie nidificanti.

\section{Haematopodidae}

\section{Beccaccia di mare Haematopus ostralegus $\mathrm{M}$ reg}

In Europa, è specie nidificante in decremento (Staneva \& Burfield, 2017). Nei periodi delle migrazioni rilevata più facilmente in complessi deltizi, presso aree fangose e zone umide (Brichetti \& Fracasso, 2004). Nel PRM, osservata quasi esclusivamente alla foce del fiume Ombrone e nella Palude della Trappola ove, specialmente per alimentarsi, frequenta le aree fangose e i cordoni sabbiosi. Le osservazioni riguardano perlopiù 1-3 individui non riproduttori o, comunque, senza poter rilevare indizi di ni- 


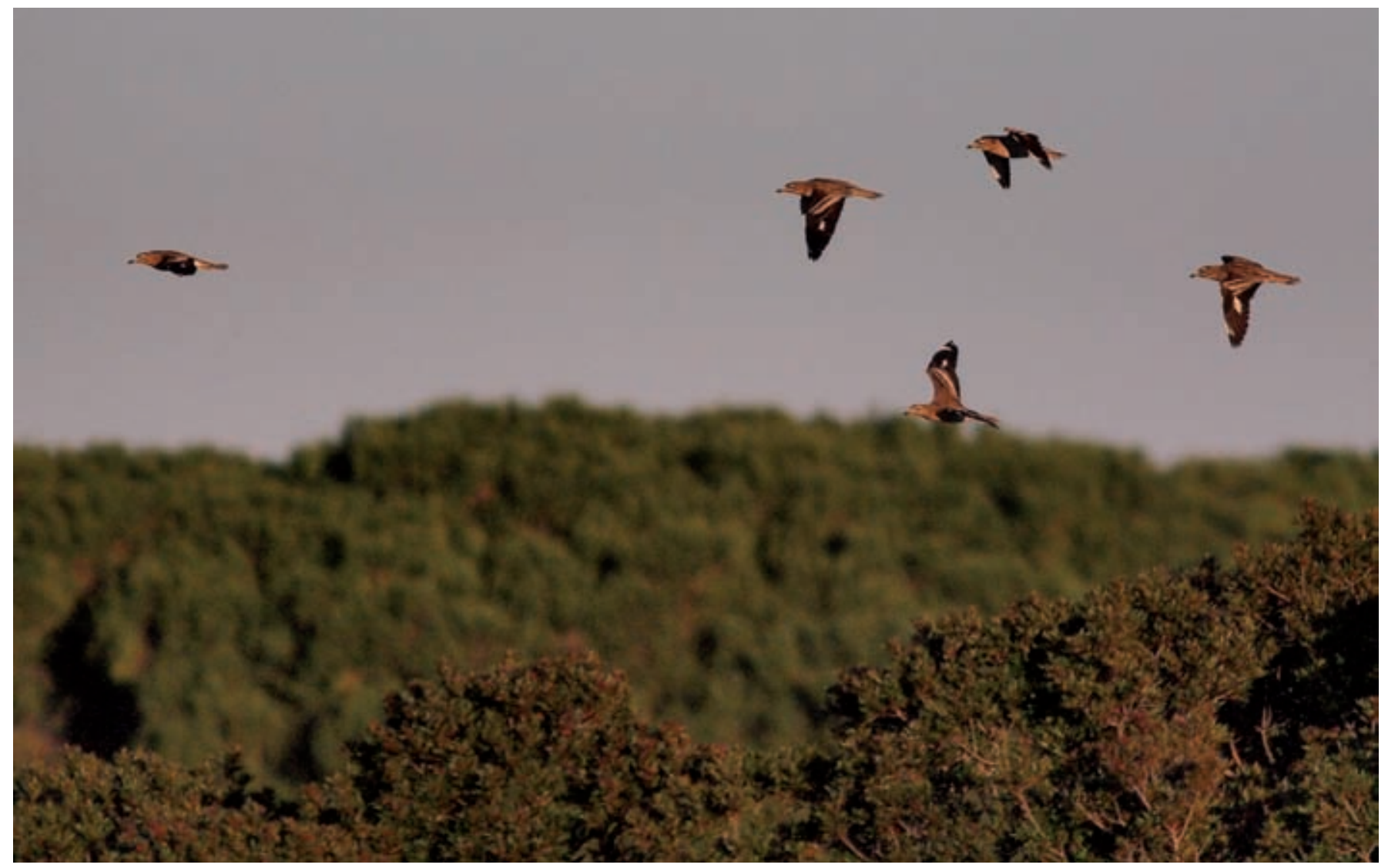

Fig. 31 - Occhioni il 07/12/2013 a Torre Trappola. / Stone-curlews on the $7^{\text {th }}$ December 2013 at Trappola Tower (Foto / Photo Stefano Laurenti).

dificazione. Concentrazioni interessanti, tuttavia riferibili a movimenti migratori: 12 ind. il 07/10/1990 alla foce del fiume Ombrone (PG); 11 ind. il 09/05/1994 alla foce del fiume Ombrone (PG); 8 ind. il 31/03/2004 nella Palude della Trappola (PG e R. Dennis). Segnalazione in periodo inusuale per l'area protetta: 1 ind. il 14/11/2011 alla foce del fiume Ombrone (M. Lunardi). Occasionale altrove: 2 ind. il 05/04/1995 in località Cannelle, Talamone (GA).

\section{Recurvirostridae}

Cavaliere d'Italia Himantopus himantopus M reg, B irr Specie stabile in Europa come nidificante (Staneva $\&$ Burfield, 2017). Si riproduce in nuclei semi-coloniali costituiti usualmente da una a poche decine di coppie, in ambienti salmastri costieri e di acqua dolce dell'interno, prevalentemente sino a $100 \mathrm{~m}$ s.l.m.; durante la migrazione rinvenuto anche in laghi e foci fluviali (Brichetti \& Fracasso, 2004). Nel PRM, è inizialmente osservato intorno alla fine della prima decade di marzo, con presenze che nell'area protetta (sino 25-35 individui) si protraggono sino alla conclusione di agosto - inizio settembre. Segnalazione più precoce: 2 ind. il 02/03/2002 alla foce del fiume Ombrone (PG). Le indicazioni di avvenuta riproduzione decorrono dal 1978 e proseguono nel 1981 (Romè, 1978; Romè \& Travison, 1982a). Sebbene la nidificazione risultasse già in quegli anni irregolare, è stato possibile accertare un nuovo caso di riproduzione nella Palude del- la Trappola, con un individuo ed un giovane non ancora in grado di volare ai margini del Chiaro del Porciatti il 21/06/1999, seguito nel giugno 2004 da un tentativo infruttuoso di almeno 6 coppie alle Saline di San Paolo e foce del fiume Ombrone (PG; GA). In questa ultima area, il ripetuto ingresso di acqua dal mare associata a repentini innalzamenti dei livelli prima della realizzazione di opere di difesa, ha facilmente compromesso il buon esito delle deposizioni. Insediamenti successivi negli anni 2005 , 2006, 2008, 2010 e 2011 con un numero variabile tra le 7-8 e 20-22 di coppie riproduttrici (Monti, 2012a; GA, F. Pezzo, PG; Fig. 32). Nel 2018 rilevata la nidificazione di 3 coppie ai margini della Palude della Trappola (PG).

\section{Avocetta Recurvirostra avosetta $\mathrm{M}$ reg, $\mathrm{W}$ irr}

Specie fluttuante in Europa come nidificante (Staneva \& Burfield, 2017). Gregaria durante tutto l'anno, frequenta specchi di acqua poco profonda, quasi sempre salata, fiancheggiati da superfici asciutte e prive di vegetazione (Brichetti \& Fracasso, 2004). Nel PRM, viene rilevata alla foce del fiume Ombrone così come nella zona umida salmastra della Palude della Trappola. La Macrozona "Maremma Grossetana" è un sito di importanza regionale e nazionale per lo svernamento della specie (Arcamone et al., 2007; Zenatello et al., 2014). Le osservazioni riguardano, in ogni circostanza, per lo più 2-3 individui in migrazione, occasionalmente sino ad inizio giugno, ma senza evidenze di nidificazione: 3 ind. il 05/06/1990 nella 


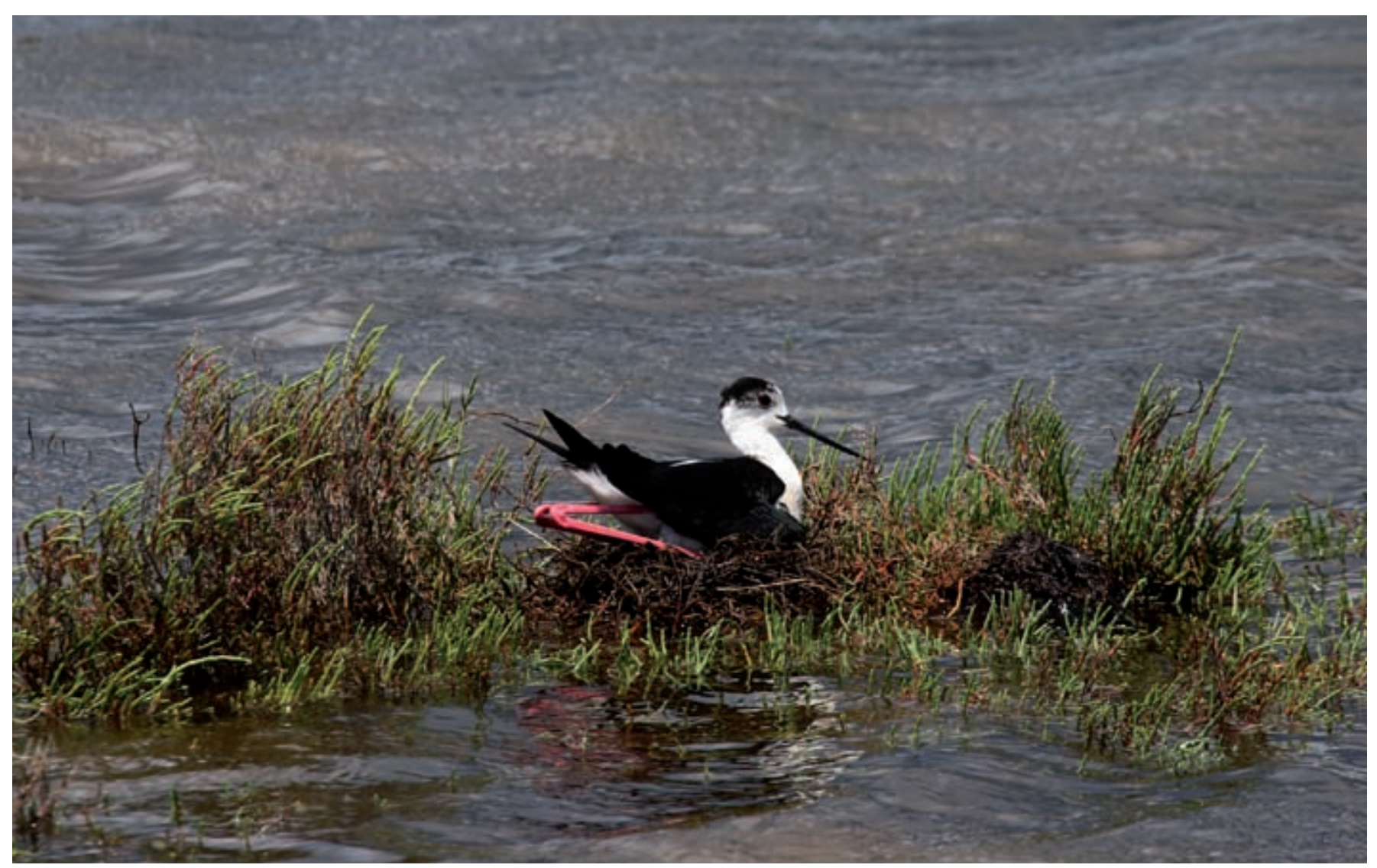

Fig. 32 - Cavaliere d'Italia in cova alle Saline di San Paolo. / Black-winged Stilt in hatching at the San Paolo Salt Flats (Foto / Photo Giuseppe Anselmi).

Palude della Trappola (PG e M. Mei); 2 ind. il 02/06/1995 alla foce del fiume Ombrone (PG e M. Lunardi). Segnalazioni in inverno piuttosto ricorrenti, secondo i dati raccolti nelle seguenti stagioni: 1995/96, 1997/98, 1998/99, 1999/00, 2009/10, 2017/18 (Monti, 2012a; PG; D. Germani; A. Picciau). In questo periodo dell'anno, osservati al massimo 6 individui il 08/12/2017 nella Palude della Trappola (PG, E. Arcamone e E. Meschini). Cifra massima registrata negli anni: 12 ind. il 13/03/1991 nella Palude della Trappola (PG).

\section{Charadriidae}

Pavoncella Vanellus vanellus $\mathrm{M}$ reg, W reg

Specie in decremento in Europa come nidificante (Staneva \& Burfield, 2017). Con le migrazioni e lo svernamento viene rilevata più comunemente nelle zone costiere con spazi aperti e suoli umidi, generalmente sino a circa $500 \mathrm{~m}$ s.l.m. (Brichetti \& Fracasso, 2004). In Toscana è anche nidificante occasionale (Mainardi, 1997a). Nel PRM, i contingenti osservati a gennaio presentano negli anni consistenze fluttuanti, con una media per stagione nel periodo 2013-2017 di 292 individui (fonte: COT, archivio PRM). La Macrozona "Maremma Grossetana" è sito di importanza regionale e nazionale per la specie (Arcamone et al., 2007; Zenatello et al., 2014). I pascoli occupati stabilmente dal bestiame, specialmente dove sia stato sparso letame, avvantaggiano la specie nella ricerca trofica, con interessanti concentrazioni rilevate nel tempo: ad es. circa 1000 ind. il 10/01/1997 alle Macchiozze (PG e M. Lunardi); 802 ind. il 07/01/2001 alle Macchiozze (PG); 600 ind. il 18/01/2014 nella Tenuta Torre Trappola (PG e A. Picciau). In periodo estivo, ripetute osservazioni di singoli individui: 1 ind. il 26/07/1993 alle Macchiozze (PG); 1 ind. il 03/08/1998 nei pascoli della Trappola (PG); 1 ind. il 02/08/2003 alla foce del fiume Ombrone (P. Arrigucci e L. Bernardini).

Piviere dorato Pluvialis apricaria $\mathrm{M}$ reg, W reg

Specie in incremento in Europa come nidificante (Staneva \& Burfield, 2017). Durante le migrazioni e lo svernamento frequenta ambienti aperti di tipo steppico, interni e costieri, sia coltivati che naturali (Brichetti \& Fracasso, 2004). In autunno-inverno, è nota nel PRM la regolare utilizzazione dei pascoli salmastri delle Saline di San Paolo anche come dormitorio notturno (GA; PG; Mainardi, 1997b). Il roost risulta occupato ad inizio ottobre: 3 ind. il 03/10/2015 (PG), più facilmente dopo la prima quindicina di novembre (Fig. 33). La Macrozona "Maremma Grossetana" è sito di importanza regionale e nazionale per la specie (Arcamone et al., 2007; Zenatello et al., 2014). Movimenti regolari all'interno dell'area protetta, con limitati spostamenti di gruppi numericamente anche consistenti all'esterno: le zone maggiormente frequentate sono infatti le Risaie di Principina, Spergolaia, Rimessini, Le Macchiozze e la Trappolaccia, per quanto l'utilizzazione av- 


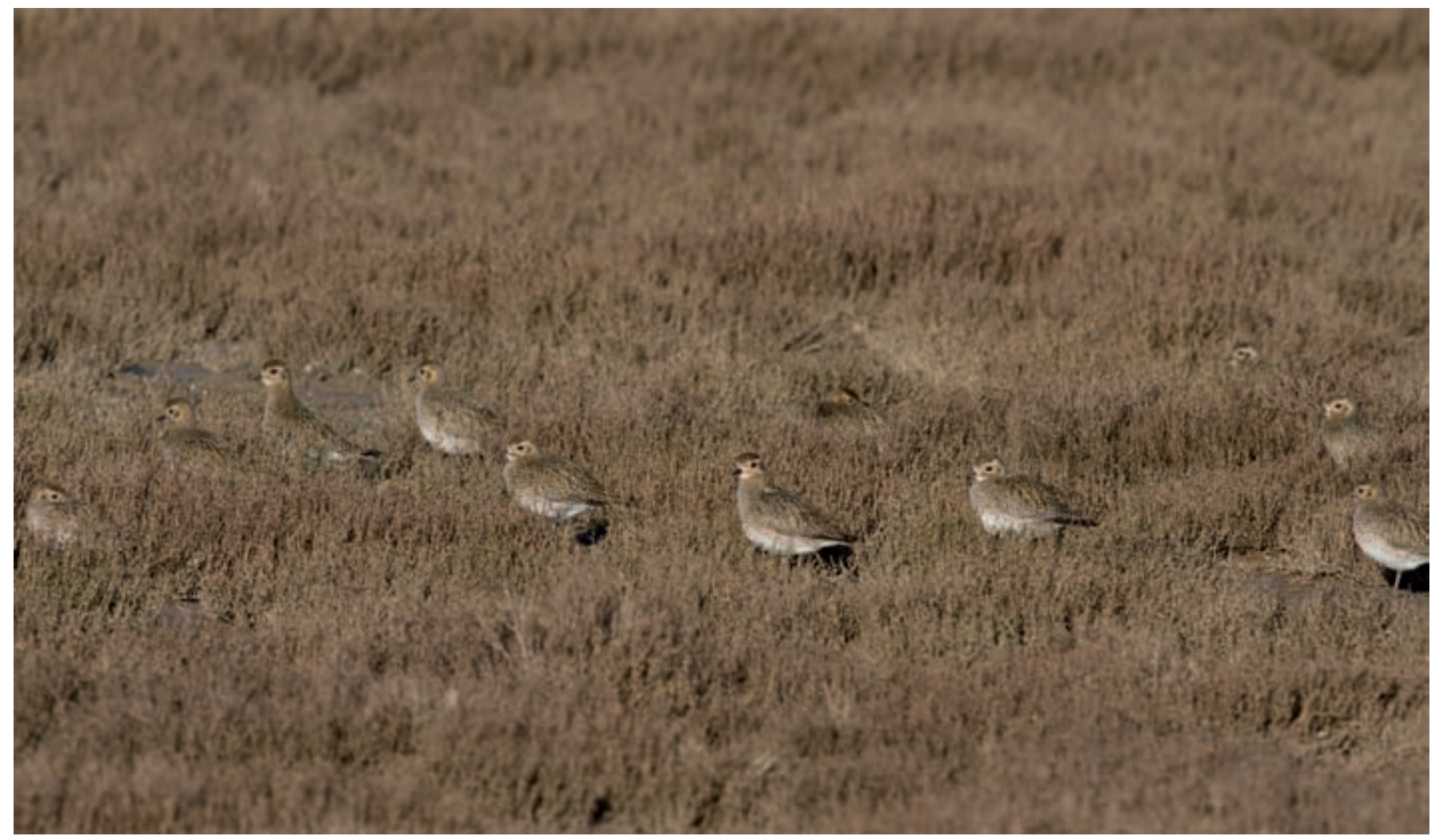

Fig. 33 - Pivieri dorati alle Saline di San Paolo. / Golden Plover at the San Paolo Salt Flats (Foto / Photo Giovanni Cappelli).

venga per ciascuna di esse in modo discontinuo (PG; GA; A. Picciau; M. Brandi; M. Dragonetti). Vengono quindi occupati seminativi, pascoli salmastri ed incolti. La media degli individui calcolata nel periodo 2013-2017 indica per l'area protetta la presenza di 186 svernanti l'anno (fonte: COT, archivio PRM). Recenti concentrazioni significative: 350 ind. il 12/02/2012 a Spergolaia (PG); 327 ind. il 06/01/2017 tra Spergolaia, foce del fiume Ombrone e Trappola (M. Carabella, PG e F. Pianezza su Ornitho.it). Presenza di 9 ind. in volo il 28/09/1969 alla Trappola (Castellani, 1970).

\section{Pivieressa Pluvialis squatarola $\mathrm{M}$ reg, $\mathrm{W}$ irr}

In Europa, è specie con trend sconosciuto come nidificante (Staneva \& Burfield, 2017). Con le migrazioni frequenta vari tipi di zone umide, soprattutto costiere; in svernamento è legata a foci fluviali in aree con forti escursioni di marea, saline e lagune ricche di banchi di fango o sabbia (Brichetti \& Fracasso, 2004). La Macrozona "Maremma Grossetana" è un sito di importanza regionale per lo svernamento della specie (Arcamone et al., 2007). Nel PRM, durante i recenti anni 2010-2017, le presenze invernali nei pascoli costieri salmastri e quelle registrate in corrispondenza dei banchi di fango alla foce del fiume Ombrone e nelle zone umide della Trappola risultano discontinue, con effettive assenze registrate durante le intere stagioni $2013 / 14$ e 2014/15 e dove, comunque, la popolazione media svernante è stata valutata in 6 individui/ anno nel periodo 2013-2017 (fonte: COT, archivio PRM; PG). Nei decenni precedenti la specie compare a gennaio con contingenti anche numericamente più consistenti:
65 ind. il 13/01/1986 nella Palude della Trappola (E. Arcamone, GA, N. Baccetti, F. Cianchi, A. Massi, E. Meschini e A. Roselli in Arcamone \& Tellini, 1987); 48 ind. il 25/12/1997 alla foce del fiume Ombrone (PG). Concentrazioni rilevanti in migrazione: 81 ind. il 03/11/1998 alla foce del fiume Ombrone (PG); 46 ind. il 15/10/2002 sulla spiagga a Principina Mare (PG). Interessanti segnalazioni per l'area in periodo estivo: 5 ind. il 02/06/1995 alla foce del fiume Ombrone (M. Lunardi e PG); 1 ind. il 24/06/1995 alla foce del fiume Ombrone (PG); 4 ind. il 01/07/1996 alla foce del fiume Ombrone (PG).

\section{Corriere grosso Charadrius hiaticula $\mathrm{M}$ reg, W irr}

In Europa, è nidificante con trend non conosciuto (Staneva \& Burfield, 2017). Specie strettamente costiera ed a distribuzione limitata in Toscana (Serra, 1997; Brichetti \& Fracasso, 2004). Nel PRM, viene rilevato alla foce del fiume Ombrone, più facilmente in corrispondenza dei banchi fangosi e dei pascoli salmastri, così come ai margini degli stagni retrodunali e nella Palude della Trappola. Occasionalmente rinvenuto nell'area del golfo di Talamone. La Macrozona "Maremma Grossetana" è sito di importanza regionale per lo svernamento della specie (Arcamone et al., 2007). In inverno è osservato in gruppi composti generalmente da 1-4 individui, sebbene la presenza nell'area protetta sia discontinua come risulta, nel periodo 2013-2017, dalla regolare assenza durante i censimenti di gennaio (fonte: COT, archivio PRM). Limitate osservazioni ad inizio dicembre: 1 ind. il 03/12/2017 alla foce del fiume Ombrone (PG e SL). Negli anni, registrati movimenti da marzo sino a fine maggio e da agosto a 
tutto novembre, normalmente riguardanti un totale di 1-6 individui. Concentrazioni inusuali in migrazione, ad es. 12 ind. il 12/03/1993 alla foce del fiume Ombrone (PG); 7 ind. il 04/09/2014 nella Palude della Trappola (PG); 8 ind. il 11/10/2015 nella Palude della Trappola (PG e F. Farsi). Occasionale ai margini esterni del PRM: 1 ind. il 29/12/2000 nel golfo di Talamone (PG). Rinvenuti alla foce del fiume Ombrone, nel mese di settembre 2014 e nel periodo agosto-settembre 2015, individui provvisti di anello in PVC $(\mathrm{n}=2)$, inanellati rispettivamente nell'agosto 2013 e luglio 2015 alle Saline di Comacchio, Ferrara (PG; ML) (fonte: ISPRA). Osservati 1 ind. il 27/12/1987 nella Palude della Trappola (A. Sorace in Arcamone \& Tellini, 1988); 2 ind. il 16/12/1988 alla foce del fiume Ombrone (S. Benucci in Arcamone \& Tellini, 1991-1992); 3 ind. il $03 / 12 / 1989$ alla foce del fiume Ombrone (N. Baccetti in Arcamone \& Tellini, 1991-1992). Segnalata la presenza di circa 25 ind. il 11/05/1969 alla Trappola (Castellani, 1970).

\section{Corriere piccolo Charadrius dubius $\mathrm{M}$ reg, W irr}

In Europa, è specie nidificante in decremento (Staneva $\&$ Burfield, 2017). Durante le migrazioni e lo svernamento frequenta, oltre a saline e litorali sabbiosi, anche pascoli salmastri, coltivi e laghi montani (Brichetti \& Fracasso, 2004). Nel PRM, rilevato lungo le sponde del fiume Ombrone, ma anche in corrispondenza dei banchi fangosi e dei pascoli salmastri, più raramente ai margini degli stagni temporanei delle Macchiozze. Si tratta di osservazioni registrate durante la migrazione, per lo più 1-3 individui da marzo a tutto il mese di maggio e da luglio a ottobre-novembre, con più recenti casi di estivazione di adulti in giugno negli anni 2012 e 2017, forse da mettere anche in rapporto con la vicina cava dismessa della Voltina, dove la specie nidifica (A. Madella su Ornitho.it; A. Picciau e PG). Svernamento di singoli individui alla foce del fiume Ombrone nelle stagioni 1990/91 e 2004/05 (Arcamone et al., 2007). Segnalato anche nel gennaio 1998, con un individuo alle Macchiozze (Biondi et al., 2000). In questo periodo dell'anno rilevato un raggruppamento inusuale: 26 ind. il 19/01/1987 nella Palude della Trappola (N. Baccetti e E. Meschini in Arcamone \& Tellini, 1988). In migrazione si segnala: 1 ind. il 09/11/1998 sulla spiaggia di Principina Mare (PG); 14 ind. il 14/03/2004 nella Palude della Trappola (PG). Occasionale altrove: 1 ind. il 21/03/2009 nel golfo di Talamone (PG).

Fratino Charadrius alexandrinus $\mathrm{M}$ reg, B, W irr

In Europa, è specie nidificante in decremento (Staneva \& Burfield, 2017). In Italia, è nidificante "In Pericolo" (Peronace et al., 2012). Durante tutto l'anno è presente prevalentemente lungo i litorali sabbiosi ben conservati, barene argillose, ai margini di zone umide costiere ed alle

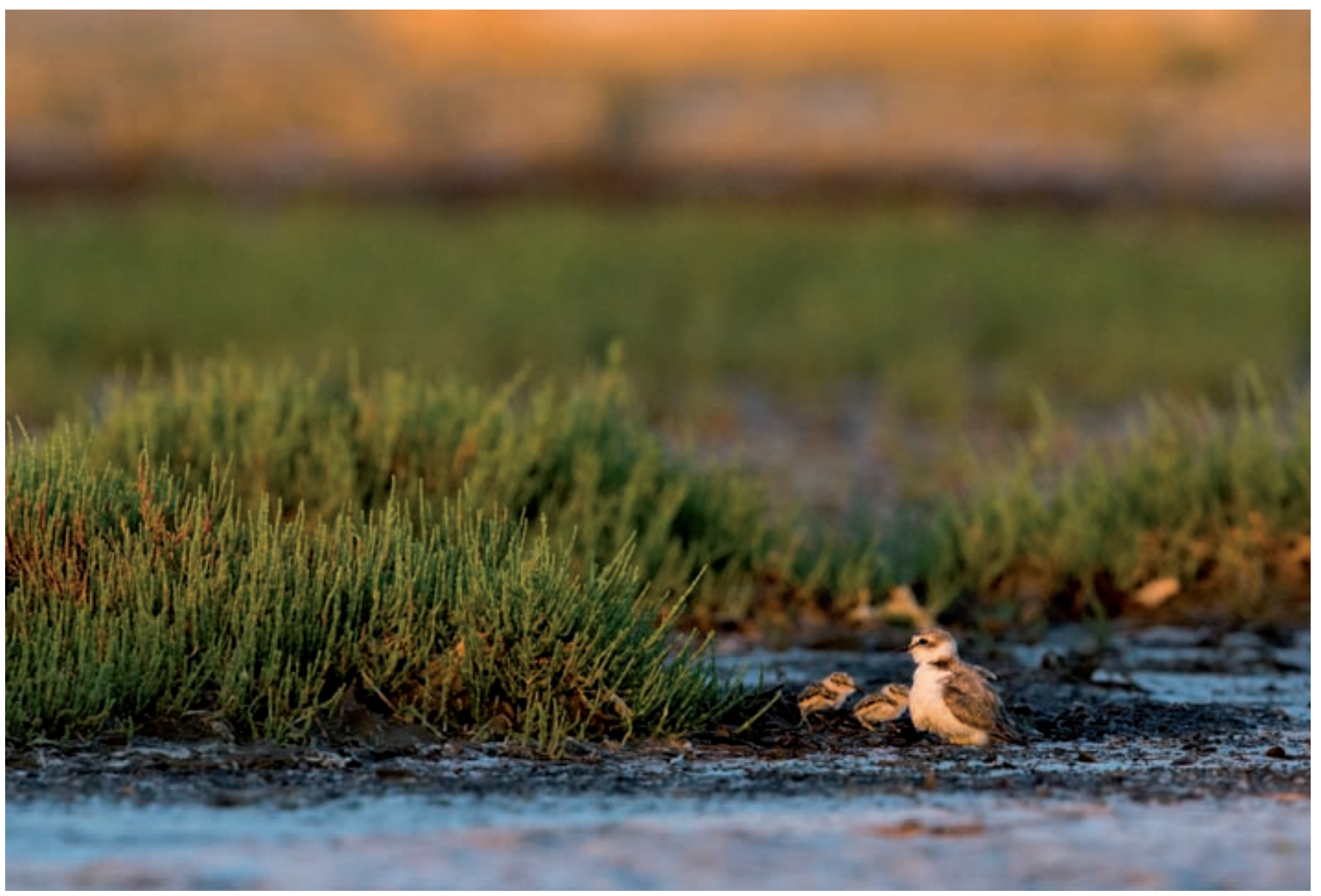

Fig. 34 - Fratino con pulcini il 04/08/2011 alla foce del fiume Ombrone. / Kentish Plover with chicks on the $4^{\text {th }}$ August 2011 at the Ombrone river mouth (Foto / Photo Alberto Pastorelli). 
foci dei fiumi; la nidificazione avviene con coppie isolate o raggruppate; le uova vengono deposte sul terreno in ambiente quasi privo di vegetazione, tra metà marzometà agosto (Brichetti \& Fracasso, 2004). La Macrozona "Maremma Grossetana" è un sito di importanza regionale per lo svernamento della specie (Arcamone et al., 2007). In fatto di habitat, nel PRM vengono preferiti i litorali sabbiosi, i pascoli salmastri e le barene argillose della foce del fiume Ombrone così come le zone soggette ad interventi per la stabilizzazione del litorale. In questa area protetta, successivamente ai dati raccolti sulla nidificazione nel 1989, si evidenzia nel 2008, in occasione di una prima nuova ricerca, il crollo della popolazione verosimilmente nella misura del $86 \%$ circa. Infatti, complessivamente: nel 1989 da Marina di Grosseto Sud a Cala Rossa rilevate 15 coppie; nel 2008 da Principina Mare sino a Cala Francese (o Cala Rossa) rinvenute 2 coppie (Mainardi, 1993-1994; Giovacchini et al., 2011). Nuove inchieste per gli anni 2009, 2014, 2015 e 2016 hanno comunque confermato la gravità della situazione in Toscana, specialmente nell'ultimo triennio con 3-5 coppie censite nel PRM (Puglisi \& Meschini, 2016). Alcuni dati interessanti: 2 ind. il 14/01/1979 nella zona del San Carlo-Trappola (C. Castellani); 1 adulto e 2 pulli il $04 / 08 / 2011$ alla foce del fiume Ombrone (A. Pastorelli; Fig. 34); 22 ind. e 7 giovani recentemente involati il 18/08/2011 insieme alla foce del fiume Ombrone (GA in GOM-List); 1 adulto e 3 pulli il 29/07/2013 in prossimità della foce del fiume Ombrone (M. Colli in GOM-List). Anche per l'area protetta è corretto evidenziare, negli anni più recenti, un successo riproduttivo scarso così come risulta ormai minima la possibilità di rilevare una produttività adeguata a sostenere la popolazione in un prossimo futuro (Puglisi \& Meschini, 2015). In inverno, in un recente passato, la specie veniva osservata regolarmente, anche in folti raggruppamenti: circa 30 ind. il 22/12/1993 nei pascoli salmastri alla foce del fiume Ombrone (PG). Nel mese di gennaio, nel periodo 2010-2017, osservati 2 individui unicamente in occasione del censimento del 2016 (fonte: COT, archivio PRM). Specialmente sul finire dello scorso secolo, nel periodo 1993-1996, presenze regolari invernali di 1-2 individui nel golfo di Talamone, immediatamente fuori dal PRM (PG; GA); 2 ind. il 07/12/2003 nel golfo di Talamone (PG). Cifre in assoluto più elevate durante la migrazione autunnale: 51 ind. il 20/08/1997 alla foce del fiume Ombrone (PG); circa 40 ind. il 27/09/2002 nella Palude della Trappola (S. Benucci e L. Vanni in EBN-Italia). Stimabile una popolazione minima di 1 coppia nidificante.

\section{Piviere tortolino Charadrius morinellus M irr}

In Europa, è specie nidificante in decremento (Staneva $\&$ Burfield, 2017). Con le migrazioni frequenta ambienti montani sommitali, ma anche coltivi di pianura, pascoli, litorali marini e margini di zone umide (Brichetti \& Fracasso, 2004). Nel PRM, è rilevato nell'area compresa tra la foce del fiume Ombrone e le Macchiozze, in ambienti caratterizzati da rada vegetazione erbacea. Le osservazioni decorrono dalla fine del secolo scorso: 1 ind. il 02/11/1997 alla foce del fiume Ombrone (PG); 2 ind. il 07/03/1998 alla foce del fiume Ombrone (M. Lausetti); 2 ind. il 23/05/2000 alle Macchiozze (Giovacchini in Arcamone \& Brichetti, 2001); 1 ind. il 16/08/2001 alla foce del fiume Ombrone (GA); 1 ind. il 30 e 31/03/2002 alla foce del fiume Ombrone (L. Vanni e P. Marotto in Arcamone \& Puglisi, 2006).

\section{Scolopacidae}

Chiurlo piccolo Numenius phaeopus $\mathrm{M}$ reg, W irr

In Europa, è specie nidificante stabile (Staneva \& Burfield, 2017). Nei periodi delle migrazioni frequenta zone umide costiere, con maggiori concentrazioni autunnali in lagune soggette a maree; in inverno appare legato a lagune, stagni salmastri, saline e foci fluviali (Brichetti \& Fracasso, 2004). Nel PRM, viene rilevato per lo più in ambienti caratterizzati da stagni salmastri ed alla foce del fiume Ombrone. Movimenti migratori nell'area protetta generalmente tra marzo-aprile e agosto-settembre. Svernamento irregolare, forse soltanto dall'inverno 1987/88 in poi; un effettivo caso registrato più recentemente con 2 ind. il 17/01/2010 (fonte: COT, archivio PRM; Arcamone, 1997b). Al contrario, le presenze invernali paiono essere più ricorrenti, ad es. 1 ind. il 09/12/1998 alla foce del fiume Ombrone (PG); 2 ind. il 04/01/1999 alla foce del fiume Ombrone (PG); 1 ind. il 29/11/2015 al dormitorio notturno di Numenius arquata, Palude della Trappola (PG). Segnalazioni estive: 1 ind. dal 03 al 29/06/1999 alla foce del fiume Ombrone (PG); 1 ind. il 03/07/2003 alla foce del fiume Ombrone (GA e PG); 1 ind. il 20/06/2009 alla foce del fiume Ombrone (PG); 1 ind. insieme a 2 ind. di Numenius arquata il 08/07/2017 nella Palude della Trappola (PG); 2 ind. insieme a 26 ind. di Numenius arquata il 23/06/2018 in un'area di raduno notturno collettivo nella Palude della Trappola (PG). Concentrazioni inusuali per l'area: 12 ind. il 26/07/1986 alle Macchiozze (GA in Arcamone \& Tellini, 1987); 19 ind. il 07/04/1997 sulla spiaggia di Principina Mare (PG).

\section{Chiurlo maggiore Numenius arquata $\mathrm{M}$ reg, W reg}

In Europa, è specie nidificante in decremento (Staneva \& Burfield, 2017). Con le migrazioni e lo svernamento appare legata a zone umide costiere, pascoli parzialmente allagati, zone fangose, foci dei fiumi; possibilità di registrare spostamenti anche a dicembre (Brichetti \& Fracasso, 2004). Nel PRM, movimenti migratori generalmente tra febbraio-aprile e luglio-novembre. Gruppi consistenti a fine inverno: oltre 200 ind. il 01/02/2013 alla foce del fiume Ombrone (ML su Ornitho.it). La Macrozona "Maremma Grossetana" è sito di importanza regionale e nazionale per lo svernamento della specie (Arcamone et al., 2007; Zenatello et al., 2014). Nel periodo 2013-2017 rilevata a gennaio una media di 69 individui svernanti per stagione (fonte: COT, archivio PRM). Alla Trappola, presenza di un roost utilizzato anche in periodo estivo, più recentemente: 26 ind. il 23/06/2018 insieme a 2 ind. di $\mathrm{Nu}$ menius phaeopus in un'area di raduno notturno collettivo (PG). Si conferma più facilmente anche l'importanza dei siti di alimentazione ubicati all'interno o nei pressi di aree sottoposte a stretto regime di protezione (Trotta, 2011). Alcuni risultati tra $i$ più recenti censimenti realizzati al roost della Palude della Trappola nel periodo invernale: 
150 ind. il 18/01/2012 (GA, M. Simoni, M. Anselmi e M. Carri); 175 ind. il 18/01/2014 (PG e A. Picciau); 183 ind. il 17/01/2015 (PG). Sempre in inverno, concentrazioni rilevanti nelle limitrofe aree di alimentazione delle Risaie di Principina, ad es. 105 ind. il 10/01/2010 (PG). Occasionale nel passato ai margini esterni del PRM, ad es. 1 ind. il 10/01/1996 nel golfo di Talamone (PG). Anche le segnalazioni estive con numeri rilevanti sono probabilmente riferibili a individui in migrazione: 44 ind. il 07/07/1986 in località Macchiozze (GA); 31 ind. il 14/06/1989 nei Chiari del San Carlo, Trappola (PG); 43 ind. il 26/07/1997 in località Macchiozze (PG).

\section{Pittima minore Limosa lapponica $\mathrm{M}$ reg, W irr}

In Europa, è specie nidificante con trend sconosciuto (Staneva \& Burfield, 2017). Durante le migrazioni ed in inverno frequenta habitat fangosi costieri, spiagge sabbiose, foci di fiumi, margini di stagni salmastri o di acqua dolce (Brichetti \& Fracasso, 2004). Nel PRM, presenze di 1-6 individui durante le migrazioni, più facilmente tra febbraio-aprile e agosto-ottobre. Occasionale lo svernamento e, in Toscana, circoscritto quasi sempre alle sole zone umide grossetane: ad es. 1 ind. il 14/01/1979 alla foce del fiume Ombrone (C. Castellani); 1 ind. il 16/01/1992 a Bocca d'Ombrone (S. Benucci e D. Biagini in Arcamone \& Puglisi, 2006); 1 ind. il 19/01/2014 nella Palude della Trappola (fonte: COT, archivio PRM). Paiono essere più frequenti le presenze invernali, ad es. 2 ind. il 07/12/1988 alla foce del fiume Ombrone (GA e PG); 1 ind. dal 23 al 25/12/1997 alla foce del fiume Ombrone (PG); 1 ind. il 21/12/2013 alla foce del fiume Ombrone (G. Radi, M. Porciani, G. Bencini e PG); 1 ind. il 04/12/2016 alla foce del fiume Ombrone (PG); 1 ind. il 08/12/2017 nella Palude della Trappola (PG, E. Arcamone e E. Meschini). Presenza estiva: 2 ind. dal 24 al 26/06/2002 alla foce del fiume Ombrone (GA e PG).

\section{Pittima reale Limosa limosa $\mathrm{M}$ reg, $\mathrm{W}$ irr}

In Europa, è specie nidificante in decremento (Staneva \& Burfield, 2017). Con le migrazioni ed in inverno la specie frequenta preferibilmente habitat fangosi, foci di fiumi, margini di stagni salmastri e pascoli parzialmente allagati (Brichetti \& Fracasso, 2004). Nel PRM, i movimenti primaverili risultano essere oggi fortemente ridimensionati. Ancora negli anni ' 80 del secolo scorso Bocca d'Ombrone era infatti considerata una delle aree chiave nel Paese per la specie (Serra \& Baccetti, 1991). Considerando i movimenti della specie a minore dettaglio, si osserva l'uso di percorsi differenti nelle due migrazioni, con una "migrazione ad arco" che comporta anche arrivi e soste precoci ad inizio anno, più spesso non riconducibili a casi di svernamento (Smit, 1986; Arcamone et al., 2007). In tale ottica, le presenze di metàfine gennaio dovrebbero essere riferite a migratori (Corsi, 1995a). Anche nell'area protetta i casi di svernamento più probabili risultano essere limitati, con osservazioni che fanno unicamente riferimento, nella zona di foce del fiume Ombrone, ai censimenti effettuati a gennaio delle stagioni 1987/88 e 1996/97 rispettivamente con 8 ed 1 individuo (Baccetti, 1997a; CUA in Arcamone \& Puglisi, 2006). Più recentemente nel PRM, nei primi anni ' 90 del secolo scorso, i movimenti paiono essere meno diluiti nel tempo, con segnalazioni quasi del tutto circoscritte ai soli mesi di febbraio-marzo e settembre. Interessanti concentrazioni per l'area: 15 ind. il 07/02/1994 alla foce del fiume Ombrone (PG); 25 ind. il 14/03/2015 nella Palude della Trappola (PG e M. Dragonetti). Presenza estiva: 1 ind. il 06/06/2008 alla foce del fiume Ombrone (GA).

Voltapietre Arenaria intrepres M irr, W irr

In Europa, è nidificante in decremento (Staneva \& Burfield, 2017). Durante le migrazioni e lo svernamento appare legato per lo più a litorali sabbiosi con aree soggette a escursione di marea; meno frequente nelle zone umide salmastre (Brichetti \& Fracasso, 2004). Nel PRM, viene rinvenuto più facilmente alla foce del fiume Ombrone e lungo i tratti di spiaggia con distese di detriti naturali di vario genere. Presenze, per quanto irregolari, riferite nella maggioranza dei casi al periodo della migrazione autunnale, normalmente con segnalazioni di singoli o 2-3 individui. In questa parte dell'anno, cifre più consistenti: 3-6 ind. il 27/09/2009 nella Palude della Trappola (S. Benucci in EBN-Italia); 4 ind. il 06/09/2013 sulla spiaggia di Principina Mare (M. Colli in GOM-List); 4 ind. il 06/09/2014 sulla spiaggia di Principina Mare (M. Colli in GOM-List; Fig. 35). In un recente passato, la migrazione primaverile è piuttosto regolare ma verosimilmente con numeri minimi: 1 ind. il 25/05/1993 alla foce del fiume Ombrone (PG); 1 ind. il 08/05/1995 alla foce del fiume Ombrone (GA); 1 ind. il 27/05/1997 alla foce del fiume Ombrone (M. Porciani e PG). Sino al 1994 svernante regolare nel PRM con un massimo di 4 individui (Arcamone et al., 2007). Successivamente, segnalazioni ripetute ma senza mostrare continuità negli anni, ad es. 2 ind. il 24 e 25/12/1998 alla foce del fiume Ombrone (PG); 1 ind. il 23/12/2002 alla foce del fiume Ombrone (L. Vanni in EBN-Italia); 3 ind. il 08/12/2008 alla foce del fiume Ombrone (M. Dragonetti). Concentrazione inusuale per l'area: 10 ind. il 27/07/1992 alla foce del fiume Ombrone (GA).

\section{Piovanello maggiore Calidris canutus $\mathrm{M}$ irr, $\mathrm{W}$ irr}

In Europa, è nidificante con trend sconosciuto (Staneva \& Burfield, 2017). Nei periodi delle migrazioni e dello svernamento appare legato a litorali sabbiosi con zone umide costiere, stagni salmastri e aree soggette a forte escursione di marea (Brichetti \& Fracasso, 2004). Nel PRM, paiono più evidenti i movimenti migratori autunnali, riconducibili al periodo agosto-ottobre, mentre risultano numericamente scarsi quelli primaverili, circoscritti ai mesi di febbraio-maggio. Osservazioni riferite alla foce del fiume Ombrone, litorale da Principina Mare a Marina di Alberese e, limitatamente, Palude della Trappola. Tra i più recenti dati: 3 ind. il 22/02/2002 alla foce del fiume Ombrone (PG); 1 ind. il 01/05/2010 a Marina di Alberese (M. Fedi su Ornitho.it); 1 ind. il 12/10/2011 alla foce del fiume Ombrone (ML su Ornitho.it); 6 ind. il 20/08/2012 alla foce del fiume Ombrone (ML su Ornitho.it); 6 ind. il 06/09/2013 a Principina Mare (M. Colli in GOM-List). Segnalazione invernale: 1 ind. il 17/01/2010 alla foce del fiume Ombrone (R. Molajoli su Ornitho.it). 


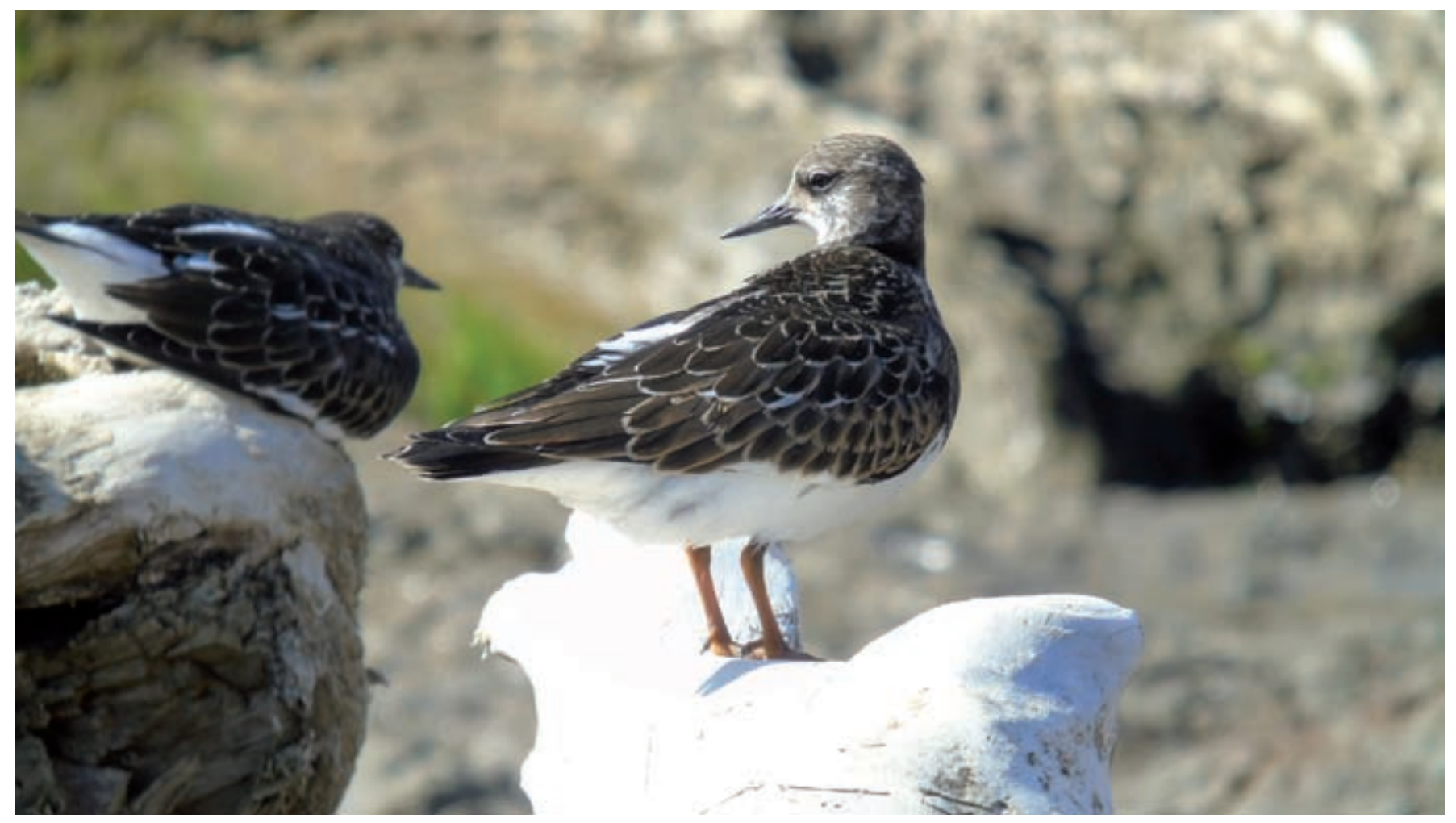

Fig. 35 - Voltapietre alla foce del fiume Ombrone. / Turnstone at the Ombrone river mouth (Foto / Photo Michelangelo Colli).

Combattente Calidris pugnax $\mathrm{M}$ reg, $\mathrm{W}$ irr

In Europa, è specie nidificante in decremento (Staneva $\&$ Burfield, 2017). Durante le migrazioni viene rilevato in zone umide costiere ed interne; in inverno la specie frequenta preferibilmente habitat fangosi, foci di fiumi, margini di stagni salmastri e pascoli parzialmente allagati (Brichetti \& Fracasso, 2004). Nel PRM, i movimenti oggi descrivono una contrazione numerica dei contingenti rispetto ad un recente passato, per quanto questi risultino più evidenti in primavera. Ancora negli anni ' 80 del secolo scorso Bocca d'Ombrone era infatti considerata una delle aree chiave nel Paese per la specie (Serra \& Baccetti, 1991). In linea di massima, come avviene per Limosa limosa, nei due momenti migratori si osserva, a un minore dettaglio di scala, l'uso di percorsi differenti, con una "migrazione ad arco" che può comportare ad inizio anno arrivi e soste precoci assimilabili a casi di svernamento (Smit, 1986; Arcamone et al., 2007). Di conseguenza, già le presenze di fine gennaio vanno riferite a migratori (Baccetti, 1997b). Nell'area protetta le osservazioni invernali ed i casi di svernamento risultano limitati, con rilevazioni che decorrono dall'inverno 1996/97: 1 ind. alla foce del fiume Ombrone il 18/01/1997 (CUA in Arcamone \& Puglisi, 2006). Altri dati invernali: 1 ind. almeno dal 1 al 05/01/2007 alla foce del fiume Ombrone (G. Paesani in Arcamone \& Puglisi, 2008; U. Mellone in EBN-Italia). Movimenti primaverili rilevati non oltre la prima decade di maggio, ad es. 34 ind. il 20/04/1996 nella Palude della Trappola (PG); 82 ind. il 14/03/2004 nella Palude della Trappola (PG e R. Dennis); almeno 7 ind. il 01/05/2010 nella zona di Marina di Alberese (M. Fedi su Ornitho.it); 4 ind. il 06/04/2011 alla foce del fiume Ombrone (C. Mar- telli in GOM-List); 3 ind. il 14/03/2015 nella Palude della Trappola (PG e M. Dragonetti). Osservazioni riferite alla migrazione autunnale, che risulta meno diluita nel tempo, ad es. 2 ind. il 28/07/1986 alle Macchiozze (GA in Arcamone \& Tellini, 1987); 25 ind. il 08/07/2000 alle Macchiozze (PG); 9 ind. il 22/07/2001 alle Macchiozze (PG); 5 ind. il 21/07/2013 alla foce del fiume Ombrone (PG). Interessante concentrazione per l'area: almeno 320 ind. il 15/03/1998 alle Macchiozze (PG). Individuo inanellato: una femmina presa alla foce del fiume Ombrone nel marzo 1969, era stata inanellata da giovane a Suomenoja, Finlandia, il 13/07/1968 (Moltoni, 1973).

\section{Gambecchio frullino Calidris falcinellus $\mathrm{M}$ irr}

In Europa, è nidificante con trend sconosciuto (Staneva \& Burfield, 2017). Durante le migrazioni frequenta preferibilmente paludi e zone fangose soggette a maree nei pressi delle foci dei fiumi (Brichetti \& Fracasso, 2004). Nel PRM, è specie precedentemente non rinvenuta (Giovacchini, 2006). Osservazioni raccolte unicamente durante la migrazione autunnale: 1 ind. il 20/08/2012 alla foce del fiume Ombrone (ML; Fig. 36); 2 ind. il 22/08/2012 alla foce del fiume Ombrone (ML). Presenze forse sottostimate.

Piovanello comune Calidris ferruginea $\mathrm{M}$ reg, W irr

In Europa, è specie svernante in incremento (Staneva $\&$ Burfield, 2017). Nei periodi di migrazione frequenta zone umide interne e costiere; in queste ultime viene specialmente osservato negli stagni, nelle saline e nelle zone intertidali (Brichetti \& Fracasso, 2004). Nel PRM, viene rinvenuto più facilmente alla foce del fiume Ombrone e 


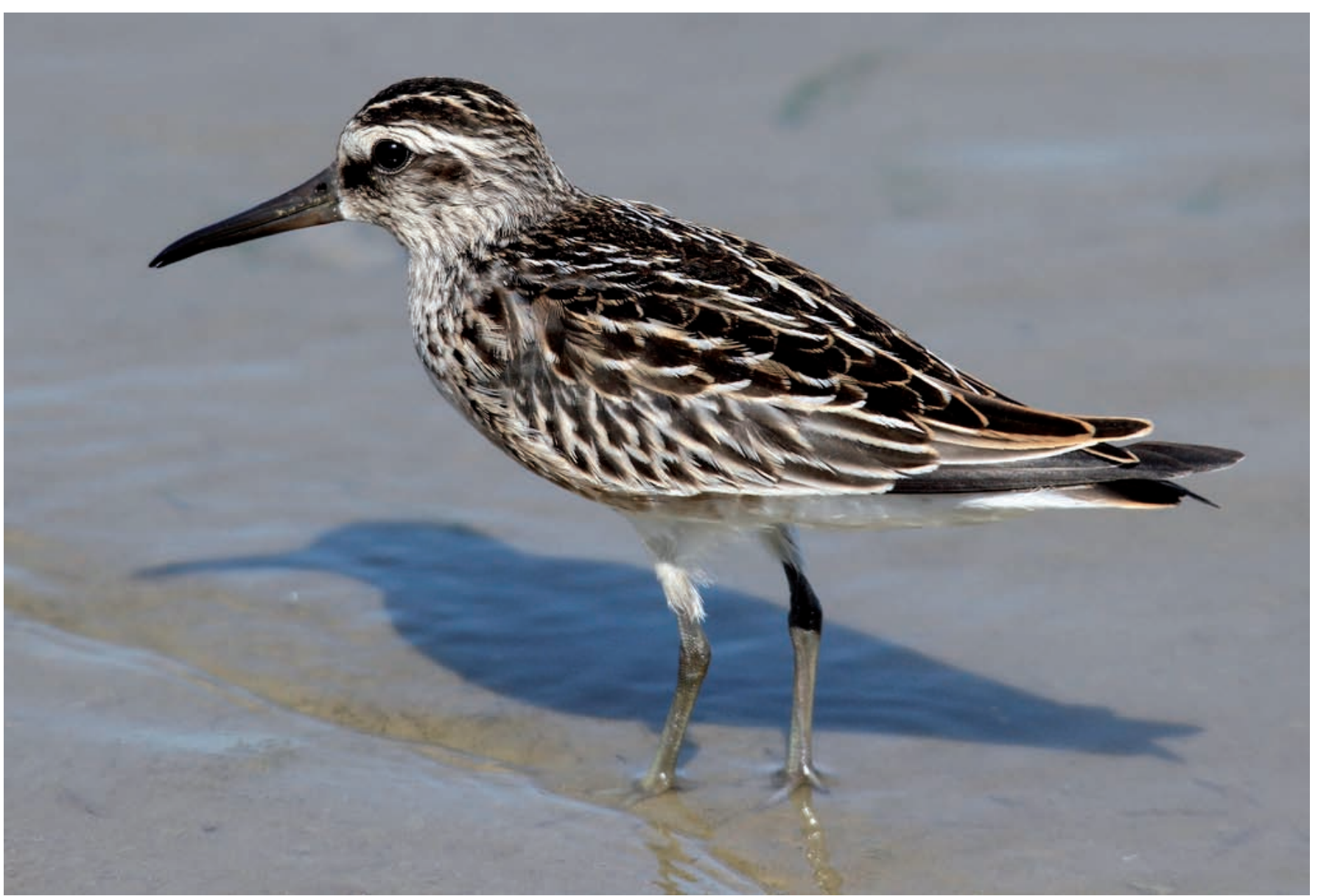

Fig. 36 - Gambecchio frullino il 20/08/2012 alla foce del fiume Ombrone. / Broad-billed Sandpiper on the $20^{\text {th }}$ August 2012 at the Ombrone river mouth (Foto / Photo Michele Lamberti).

lungo i tratti di spiaggia meno disturbati, anche in presenza di distese di detriti trasportati dal mare. Presenze riferite nella maggioranza dei casi a piccoli gruppi osservati durante le migrazioni composti da 2-3 individui. Tra le segnalazioni più interessanti: 3 ind. di cui 1 in abito nuziale il 25/05/1993 alla foce del fiume Ombrone (PG); 3 ind. il 01/05/2001 alle Macchiozze (PG); 2 ind. il 18/09/2008 lungo spiaggia a Principina Mare (GA); almeno 8 ind. il 09/09/2013 alla foce del fiume Ombrone (G. Cazzola e A. Maggioni su Ornitho.it). Presenza in periodo estivo: 4 ind. il 29/06/2015 nella Palude della Trappola (PG). Primo caso di svernamento in Toscana: 1 ind. il 11/01/2003 nella Palude della Trappola (G. Paesani in Arcamone, 2005). Concentrazione inusuale per l'area: 14 ind. di cui 3 in abito nuziale il 28/05/1999 alla foce del fiume Ombrone (PG e E. Valentini).

\section{Gambecchio nano Calidris temminckii $\mathrm{M}$ irr}

In Europa, è nidificante con trend stabile (Staneva \& Burfield, 2017). Durante le migrazioni appare maggiormente legato a zone umide costiere ed interne, preferibilmente d'acqua dolce, mentre durante l'inverno frequenta saline, stagni e ambienti di costa con substrati fangosi e parzialmente allagati (Brichetti \& Fracasso, 2004). Nel PRM, è specie precedentemente non valutata per la checklist (Giovacchini, 2006). Osservazioni riferite alla migra- zione autunnale: 1 ind. il 22/08/2002 alla foce del fiume Ombrone (M. Altieri in EBN-Italia); 3 ind. il 30/08/2002 alla foce del fiume Ombrone (F. Acerbi e L. Vanni in EBN-Italia). Per la stessa località, successive segnalazioni di individui in migrazione nel periodo 2008-2010 (Monti, 2012a). Presenze forse sottostimate per via delle piccole dimensioni che ne rendono difficile l'avvistamento.

\section{Piovanello tridattilo Calidris alba $\mathrm{M}$ reg, $\mathrm{W}$ irr}

In Europa, è nidificante con trend sconosciuto (Staneva \& Burfield, 2017). Durante le migrazioni e lo svernamento frequenta litorali sabbiosi, lagune e foci fluviali, anche in vicinanza di zone umide salmastre o saline (Brichetti \& Fracasso, 2004). Nel PRM, è specie rilevata con scarsi contingenti anche in migrazione, per lo più di 1-3 individui, lungo la spiaggia di Principina Mare sino alla foce del fiume Ombrone. Tra le segnalazioni si ricordano: 3 ind. il 15/11/1998 alla foce del fiume Ombrone (M. Lausetti); 1 ind. il 27/09/2002 nella zona della Palude della Trappola (S. Benucci e L. Vanni in EBN-List); 3 ind. il 27/10/2002 alla foce del fiume Ombrone (R. Gildi); 1 ind. il 12/09/2009 alla foce del fiume Ombrone (PG); 1 ind. il 07/09/2016 lungo la spiaggia di Principina Mare (M. Colli in GOM-List). Svernante irregolare: 1 ind. il 16/01/1999 alla foce del fiume Ombrone (CUA in Arcamone \& Puglisi, 2006); 
7 ind. il 07/12/2008 alla foce del fiume Ombrone (F. Monti, com. pers; Monti, 2012a). Concentrazione inusuale per l'area: 9 ind. il 26/04/1999 alla foce del fiume Ombrone (PG).

\section{Piovanello pancianera Calidris alpina $\mathrm{M}$ reg, $\mathrm{W}$ reg}

In Europa, è nidificante con trend sconosciuto (Staneva \& Burfield, 2017). Nei periodi di migrazione viene rilevato in vari tipi di zone umide costiere come lagune, stagni retrodunali, foci fluviali, spiagge sabbiose, così come è regolare osservarlo anche in zone umide dell'interno; durante lo svernamento appare maggiormente legato alle foci dei fiumi, specialmente nei contesti con substrati fangosi, lagune e stagni (Brichetti \& Fracasso, 2004). È il limicolo costiero svernante più numeroso in Toscana (Arcamone et al., 2007). La Macrozona "Maremma Grossetana" è un sito di importanza regionale e nazionale per lo svernamento della specie (Arcamone et al., 2007; Zenatello et al., 2014). Nel PRM, è osservabile anche in gruppi numerosi (oltre 20-30 individui) ed è facilmente rinvenuto in associazione con altri caradriformi. Negli anni risulta soggetto a fluttuazioni numeriche con una media, nel periodo 2013-2017, di circa 34 individui svernanti per stagione (fonte: COT, archivio PRM). In condizioni di marea meno favorevoli e verosimilmente per finalità trofiche, anche gli individui legati all'area protetta compiono spostamenti per utilizzare altre zone umide limitrofe. Il 16/01/1991 censiti 160 ind. svernanti nel PRM; 225 ind. il 17/01/2010 alla foce del fiume Ombrone (Arcamone et al., 1994; fonte: COT, archivio PRM). In migrazione è rinvenuto anche con numeri consistenti per singoli gruppi: circa 200 ind. il 24/03/1994 alla foce del fiume Ombrone (PG); 188 ind. 02/03/2011 alla foce del fiume Ombrone (PG). Presenza in periodo estivo: 1 ind. il 27/06/2000 alla foce del fiume Ombrone (PG). Individui inanellati: 1 ind. inanellato il 05/08/1987 alla foce della Vistola, Polonia, ricatturato il 17/01/1993 alla foce del fiume Ombrone; 1 ind. inanellato il 17/09/1993 alla foce del Reda, Polonia, ricatturato il 29/12/1993 alla foce del fiume Ombrone (Valtriani, 1994). Fedeltà al sito di svernamento: 1 ind. inanellato il 17/01/1992 alla foce del fiume Ombrone, ricatturato il 17/01/1993 e il 29/12/1993 (Valtriani, 1994).

Gambecchio comune Calidris minuta M irr ?, W irr

In Europa, è nidificante con trend stabile (Staneva \& Burfield, 2017). Nei periodi di migrazione e svernamento è rilevato di frequente in zone umide costiere come lagune, stagni salmastri e foci fluviali; nell'interno è osservato anche in risaie, bacini artificiali e rive fangose di laghi (Brichetti \& Fracasso, 2004). La Macrozona "Maremma Grossetana" è un sito di importanza regionale per lo svernamento della specie (Arcamone et al., 2007). Nel PRM, oltre che alla foce del fiume Ombrone, si rinviene anche lungo i banchi fangosi e nella Palude della Trappola. Specie generalmente contattata con un numero limitato di individui, per lo più durante le migrazioni, ad es. 7 ind. il 27/05/1997 nella Palude della Trappola (M. Porciani e PG); 1 ind. il 27/09/2003 nella Palude della Trappola (S. Benucci in EBN-Italia); 3 ind. il 17/04/2009 alla foce del fiume Ombrone (PG); 1 ind. il 09/09/2013 alla foce del fiume Ombrone (G. Cazzola e A. Maggioni su Ornitho.it). Contingenti forse sottostimati in inverno. Ripetute osservazioni a dicembre e casi di svernamento nell'area protetta rilevati almeno dalla metà degli anni ' 80 del secolo scorso: 15 ind. il 13/01/1986 nella Palude della Trappola (GA, E. Arcamone, F. Cianchi e A. Roselli in Arcamone \& Tellini, 1987; Arcamone et al., 2007). Altre osservazioni invernali tra quelle più recenti: 1 ind. il 21/12/2011 alla foce del fiume Ombrone (S. Benucci e P. M. Politi in EBN-Italia); 11 ind. il 04/12/2016 alla foce del fiume Ombrone (PG). Concentrazione inusuale per l'area: 35 ind. il 19/02/1989 alla foce del fiume Ombrone (D. Occhiato e F. Pieri in Arcamone \& Tellini, 1991-1992).

\section{Limnodromo pettorossiccio Limnodromus scolopaceus A-1}

Accidentale. 1 ind. il 26/09/1985 alla foce del fiume Ombrone (GA e F. Favali; Fig. 37). Prima segnalazione di presenza certa in Italia, omologata dalla COI, dopo un'altra riferita al Lazio, ma ritenuta dubbia (Moltoni \& Brichetti, 1978; Brichetti et al., 1986).

\section{Beccaccia Scolopax rusticola $\mathrm{M}$ reg, W reg}

In Europa, è specie nidificante stabile (Staneva \& Burfield, 2017). Durante le migrazioni e lo svernamento frequenta anche i boschi collinari e litoranei, con sottobosco arbustivo, nonchè pascoli e prati utilizzati come aree di alimentazione notturna (Brichetti \& Fracasso, 2004). Le scarse osservazioni registrate nel PRM sono più facilmente imputabili alla mancanza di specifiche indagini che ne accertino, in particolar modo, la distribuzione come svernante. Osservazioni più recenti: 1 ind. il 01/11/2009 alle Macchiozze (M. Dragonetti e PG); 1 ind. il 04/12/2013 allo Scoglietto, Alberese (PG). Nella stessa zona, registrato un incremento delle segnalazioni durante la stagione invernale 2018/19 (U. Boldorini). Individuo inanellato: 1 ind. catturato il 24/03/1998 a Sisak, Croazia, era stato inanellato il 05/01/1995 in località Lasco di Alberese, PRM (S. Spanò, com. pers.; archivio PRM).

\section{Frullino Lymnocryptes minimus $\mathrm{M}$ irr, $\mathrm{W}$ irr}

In Europa, è specie nidificante stabile (Staneva \& Burfield, 2017). Durante le migrazioni e lo svernamento frequenta zone umide di acqua dolce interne e costiere, anche debolmente salmastre (Brichetti \& Fracasso, 2004). Specie con abitudini elusive e crepuscolari, quindi più facilmente sottostimabile. Nel PRM, è stato rilevato quasi esclusivamente nelle più estese zone umide salmastre della Palude della Trappola. Lo svernamento pare essere irregolare, con segnalazioni di singoli individui che comunque sono stati rilevati sino al censimento del gennaio 2015 (Arcamone et al., 2007; fonte: COT, archivio PRM). Presenza invernale: 1 ind. il 31/12/2016 alla foce del fiume Ombrone (P. Marotto su Ornitho.it). Segnalazione precedente: 1 ind. il 22/10/1995 nella Palude della Trappola (GA); le seguenti forse riferite allo stesso animale: 1 ind. il 30/11/2005 alla foce del fiume Ombrone (GA); 1 ind. il 28/02/2006 alla foce del fiume Ombrone (R. Gildi in Arcamone \& Puglisi, 2008). 


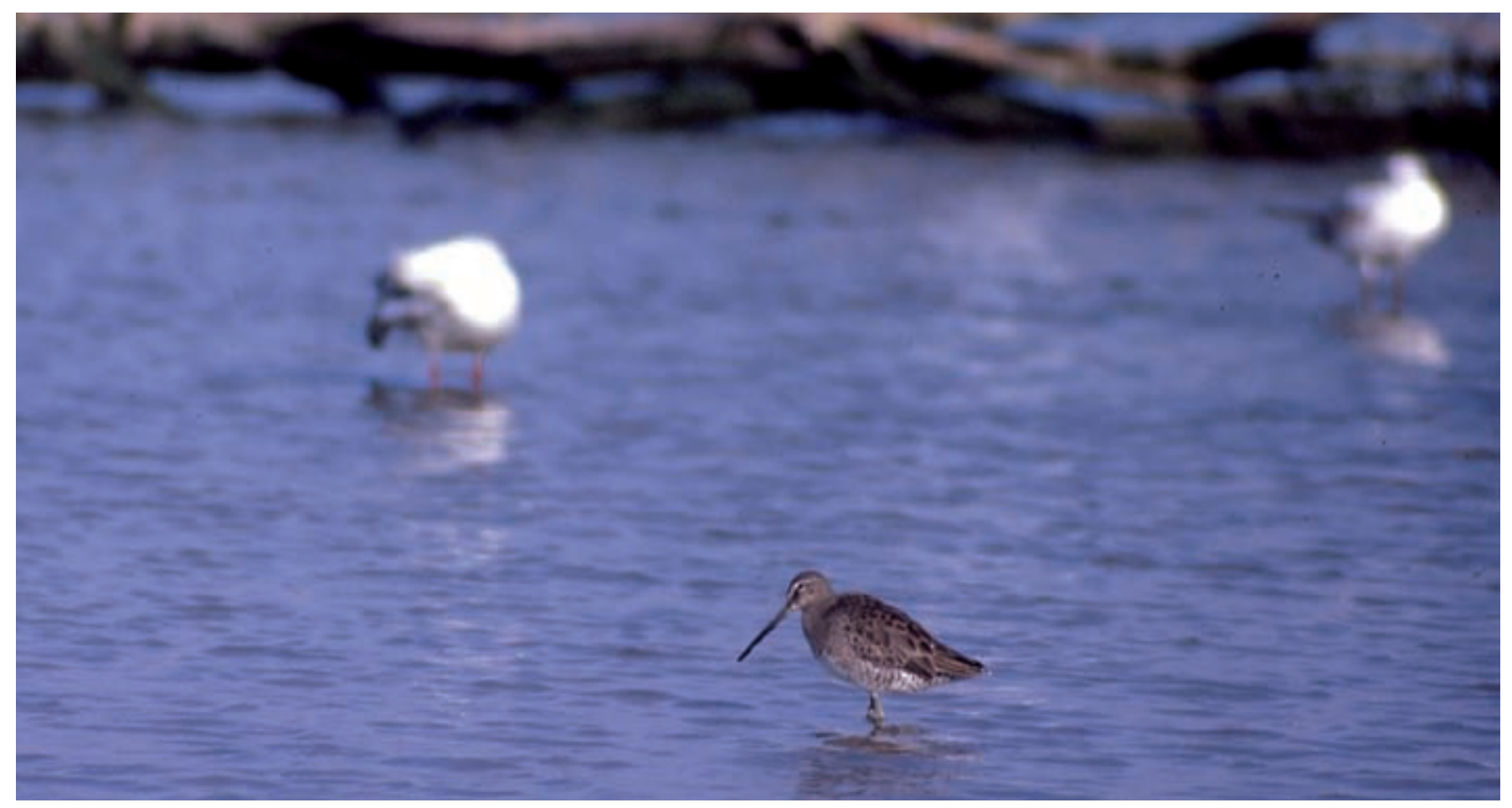

Fig. 37 - Limnodromo pettorossiccio il 26/09/1985 alla foce del fiume Ombrone. / Long-billed Dowitcher on the $26^{\text {th }}$ September 1985 at the Ombrone river mouth (Foto / Photo Giuseppe Anselmi e Fabio Favali).

\section{Croccolone Gallinago media A-1}

Accidentale. 1 ind. il 07/12/1996 in prossimità degli stagni delle Macchiozze (D. Occhiato, A. Corso e R. Gildi). Primo caso di presenza invernale certa in Italia (Moltoni \& Brichetti, 1978; Arcamone \& Brichetti, 1997).

\section{Beccaccino Gallinago gallinago $\mathrm{M}$ reg, W reg}

In Europa, è nidificante in decremento (Staneva \& Burfield, 2017). Nei periodi delle migrazioni e dello svernamento gli ambienti preferiti sono i prati parzialmente sommersi e pascolati, bordi di stagni, fiumi e canali (Brichetti \& Fracasso, 2004). La Macrozona "Maremma Grossetana" è sito di importanza regionale e nazionale per lo svernamento della specie (Arcamone et al., 2007; Zenatello et al., 2014). Nel PRM, viene rilevato più facilmente nei pascoli moderatamente allagati di Spergolaia, Macchiozze così come alla Trappola sino alle Tenute di San Carlo e delle Femminelle ed ancora in prossimità di aree di raccolta dello stallatico, negli incolti, sempre se associati a suoli umidi o fangosi, e ai margini dei canali di regimazione idraulica di Alberese, Collecchio e Talamone. Si tratta di una specie elusiva che nell'area protetta mostra una distribuzione non definibile con precisione. Nel periodo 2013-2017, nel PRM si stima una media di 50 svernanti all'anno (fonte: COT, archivio PRM). Individuo inanellato: 1 ind catturato a Valle Trappola il 14/12/1972 era stato inanellato il 23/07/1972 ad Helgoland, Germania (GIL, ined.).

\section{Piro piro piccolo Actitis hypoleucos $\mathrm{M}$ reg, $\mathrm{W}$ reg}

In Europa, è specie nidificante in decremento (Staneva $\&$ Burfield, 2017). Con le migrazioni si rinviene in vari tipi di zone umide interne così come in quelle costiere; duran- te lo svernamento è osservato più facilmente lungo costa ovvero alle foci dei fiumi ove occupa rilevati fangosi, saline, paludi, lagune ma anche i bordi dei canali di bonifica (Brichetti \& Fracasso, 2004). La Macrozona "Maremma Grossetana" è un sito di importanza regionale per lo svernamento della specie (Arcamone et al., 2007). Nel PRM, la distribuzione trattandosi di una specie piuttosto elusiva potrebbe risultare più ampia, specialmente durante le migrazioni, se potesse essere applicato un maggiore sforzo di rilevamento. In questo periodo segnalazioni riferite per lo più a singoli individui, rinvenuti anche ai margini esterni dell'area protetta, ad es. 1 ind. il 28/09/1996 nel golfo di Talamone (PG); 1 ind. il 02/03/2015 nella Palude della Trappola (PG). Interessante concentrazione: oltre 100 ind. il 26/07/1995 alla foce del fiume Ombrone (PG). Osservazione invernale tra le più recenti: 2 ind. il 04/12/2016 alla foce del fiume Ombrone (PG). Nel PRM, rilevati a gennaio nel periodo 2013-2017 una media di circa 2 individui svernanti per stagione (fonte: COT, archivio PRM).

\section{Piro piro culbianco Tringa ochropus $\mathrm{M}$ reg, $\mathrm{W}$ irr}

In Europa, è specie nidificante con trend orientato verso la stabilità (Staneva \& Burfield, 2017). Durante le migrazioni e lo svernamento frequenta zone umide con acqua dolce compresi canali di bonifica, cave, risaie, torbiere, mentre risulta più scarso lungo costa, principalmente nelle zone umide salmastre (Brichetti \& Fracasso, 2004). Nel PRM, è migratore con contingenti già presenti intorno a metà giugno. Concentrazioni di riferimento per l'area, ad es. 4 ind. il 10/09/1994 alla foce del fiume Ombrone (PG); 6 ind. il 07/04/1997 nel Canale della Bonifica di Talamone (M. Porciani e GA); 6 ind. il 18/06/2016 nella Palude della Trappola (PG e A. Picciau). Nell'area pro- 
tetta, svernamento a cominciare dalla stagione 1997/98: 1 ind. il 17/01/1998 nella Palude della Trappola (PG e G. Tellini Florenzano). Successivamente, a partire dall'inverno 2003/04, si registrano con maggiore frequenza segnalazioni invernali e nuovi casi di svernamento, anche in aree marginalmente esterne, ad es. 1 ind. il 17/01/2004 nel Canale di Bonifica di Talamone (fonte: COT, archivio PRM); 1 ind. il 12/01/2005 lungo il fiume Ombrone (PG); 1 ind. il 24/01/2010 nella zona di Alberese (M. Gagliardone in EBN-Italia); 1 ind. il 08/01/2011 alle Macchiozze (D. Brendan su Ornitho.it); 2 ind. il 15/12/2012 alla cava dismessa della Voltina (ML, A. Cappelli e A. Santi); 1 ind. il 27/12/2014 alla foce del fiume Ombrone (M. Biondi, PG, SL e E. Savo su Ornitho.it); 1 ind. il 22 e 24/12/2018 tra il fosso Razzo, Palude della Trappola, e le Risaie di Principina (PG e A. Picciau).

\section{Pettegola Tringa totanus $\mathrm{M}$ reg, W reg}

In Europa, è specie nidificante in decremento (Staneva \& Burfield, 2017). Durante le migrazioni ed in inverno frequenta le paludi e le lagune costiere, saline, spiagge, zone fangose spesso nei pressi delle foci dei fiumi (Brichetti \& Fracasso, 2004). La Macrozona "Maremma Grossetana" è sito di importanza regionale e nazionale per lo svernamento della specie (Arcamone et al., 2007; Zenatello et al., 2014). Nel PRM, rilevati a gennaio nel periodo 2013-2017 una media di circa 18 individui svernanti per stagione (fonte: COT, archivio PRM). Nell'area protetta viene quasi sempre rinvenuta con singoli individui o con gruppetti composti da 2-4 individui. Raduni notturni di più individui, anche con Tringa erythropus, nei pascoli salmastri della Palude della Trappola: 8 ind. il 16/12/2017 (PG). Con riferimento ad un recente passato, concentrazioni invernali di un certo interesse per il sito: 56 ind. il 05/01/1997 alla foce del fiume Ombrone (PG); 25 ind. dal 25 al 29/12/1999 nella Palude della Trappola (PG). Anche in migrazione contingenti a volte cospicui, ad es. 70 ind. il 15/07/1996 alla foce del fiume Ombrone (PG); 38 ind. il 16/02/1998 alla foce del fiume Ombrone (PG). Interessanti concentrazioni per l'area in estate: 8 ind. il 24/06/1994 alla foce del fiume Ombrone (PG); 9 ind. il 29/06/1998 alla foce del fiume Ombrone (PG). Individui inanellati: 1 ind. catturato a Talamone, nel novembre 1964, era stato inanellato da giovane a Amager, Danimarca, il 07/07/1962 (Moltoni, 1966); 1 ind. catturato alla foce del fiume Ombrone, Grosseto, nel marzo 1969, era stato inanellato da giovane a Aflandshage, Danimarca, il 04/07/1967 (Moltoni, 1973).

\section{Albastrello Tringa stagnatilis $\mathrm{M}$ irr, W irr}

In Europa, è specie nidificante con trend sconosciuto (Staneva \& Burfield, 2017). Con le migrazioni ed in inverno frequenta preferibilmente paludi e zone fangose soggette a maree nei pressi delle foci dei fiumi; localmente anche risaie e zone umide artificiali (Brichetti \& Fracasso, 2004). Nel PRM, compare irregolarmente in migrazione, più spesso con singoli individui ed occasionalmente a coppie: 2 ind. il 30/08/2013 alla foce del fiume Ombrone (E. Rigamonti su Ornitho.it). Segnalazione invernale di individui nella stagione 1983/84: 2 ind. il 13/01/1984 nella Palude della Trappola (M. Pani, ined.). Indicazione di presenza più recente: 1 ind. il 28/12/2015 alla foce del fiume Ombrone (PG, A. Picciau, F. Ferragamo Visconti e G. Visconti). Osservazioni di migratori ancora a novembre: 2 ind. il 02/11/1991 alla foce del fiume Ombrone (L. Becherucci in Arcamone \& Barbagli, 1995-1996); 1 ind. il 02/11/2011 alla foce del fiume Ombrone (PG).

\section{Piro piro boschereccio Tring a glareola $\mathrm{M}$ reg}

In Europa, è specie nidificante con trend discontinuo (Staneva \& Burfield, 2017). Nei periodi delle migrazioni frequenta zone umide con acqua dolce come laghetti di caccia, risaie ed anche canali di bonifica, mentre risulta più scarso nelle zone umide salmastre (Brichetti \& Fracasso, 2004). Nel PRM, segnalazioni da inizio aprile e maggio, generalmente di 1-9 individui, osservati alle Macchiozze, foce del fiume Ombrone, Palude della Trappola e nei pascoli parzialmente allagati delle Tenute di San Carlo e delle Femminelle. Risulta raro nei successivi mesi di giugno e luglio, anche con l'occupazione delle Risaie di Principina, da poco inondate: 7 ind. il 04/06/2007 alle Risaie di Principina (PG); 3 ind. il 09/07/2014 nella Palude della Trappola (PG) (Fig. 38). Scarso in migrazione in autunno con un massimo di 2 individui rilevati: 2 ind. il 19/10/2008 nella Palude della Trappola (GA). Dato pregresso: 100 ind. il 19/04/1970 alla Trappola (C. Castellani, ined.).

\section{Totano moro Tringa erythropus $\mathrm{M}$ reg, W irr}

In Europa, è specie nidificante in decremento (Staneva \& Burfield, 2017). Nei periodi delle migrazioni frequenta zone umide di acqua salmastra o dolce, costiere e interne; in inverno rilevata più facilmente in acque costiere come saline, lagune, stagni retrodunali, foci fluviali e prospicienti zone fangose (Brichetti \& Fracasso, 2004). Nel PRM, presenze concentrate con regolarità specialmente

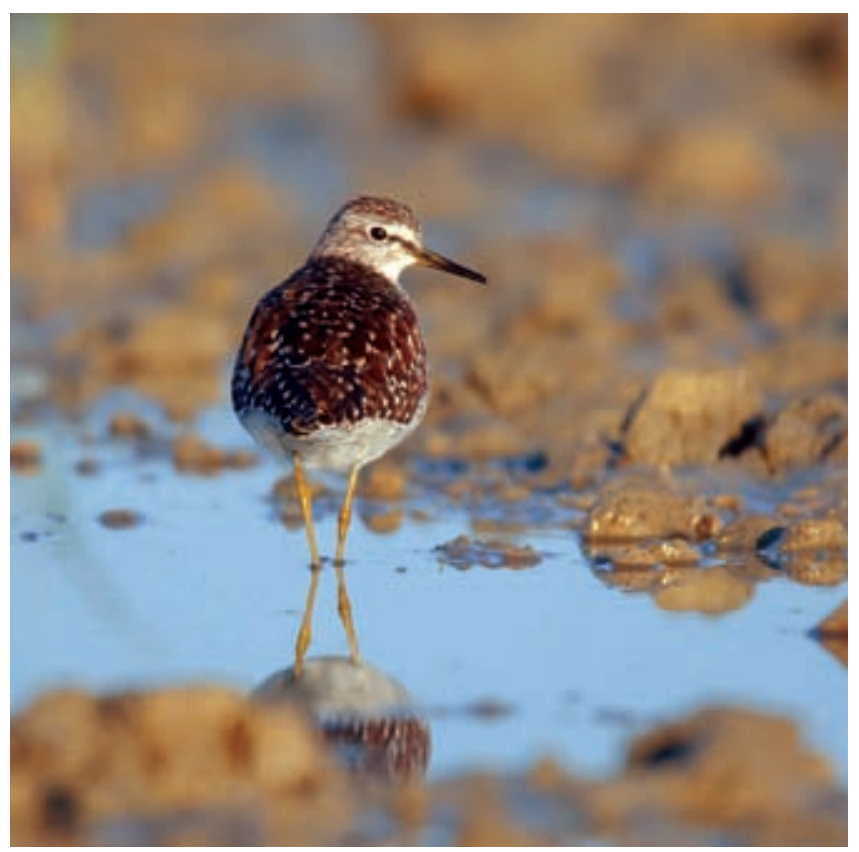

Fig. 38 - Piro piro boschereccio nelle Risaie di Principina. / Wood Sandpiper in the Principina rice fields (Foto / Photo Marco Brandi). 
durante la migrazione autunnale. Alla Trappola rilevati 5 ind. il 14/01/1979 (C. Castellani). La Macrozona "Maremma Grossetana" è sito di importanza regionale e nazionale per lo svernamento della specie (Arcamone et al., 2007; Zenatello et al., 2014). Nell'area protetta svernamento irregolare nel periodo 2013-2017, con un totale di 20 individui censiti (fonte: COT, archivio PRM). Raduni notturni di più individui nei pascoli salmastri della Palude della Trappola: almeno 6 ind. il 16/12/2017 (PG). In un recente passato, contingenti invernali più cospicui: 23 ind. il 18/01/1997 (fonte: COT, archivio PRM); 21 ind. il 30/12/2000 nella Palude della Trappola (GA, G. F. Martini e PG). Occasionali osservazioni di singoli individui in periodo estivo.

\section{Pantana Tringa nebularia $\mathrm{M}$ reg, W reg}

In Europa, è specie nidificante con trend incerto (Staneva \& Burfield, 2017). Nei periodi delle migrazioni frequenta zone umide di acqua dolce o salmastra, con regolari presenze in risaie; con lo svernamento utilizza saline, stagni costieri e lagune (Brichetti \& Fracasso, 2004). Presenze invernali lungo la fascia costiera tirrenica scarse ed irregolari sino alla metà circa degli anni ' 90 del scorso secolo. La Macrozona "Maremma Grossetana" è un sito di importanza regionale per lo svernamento della specie (Arcamone et al., 2007). Nel PRM, analoga situazione anche durante le migrazioni dove pare essere rinvenuto con regolarità a partire dal 1994: 1 ind. il 28/04/1994 alla foce del fiume Ombrone (PG). In questo periodo sono osservati più facilmente singoli esemplari o gruppetti per un massimo di 3 individui, unicamente nelle zone della foce del fiume Ombrone e nella Palude della Trappola. Concentrazioni superiori, meritevoli di segnalazione per l'area, ad es. 12 ind. il 09/09/1996 alla foce del fiume Ombrone (PG); 8 ind. il 14/10/2002 alla foce del fiume Ombrone (PG); 6 ind. il 04/09/2014 nella Palude della Trappola (PG). Prime segnalazioni raccolte in inverno nell'area protetta: 1 ind. il 18/01/1997 nella Palude della Trappola (GA); 1 ind. il 01/12/1997 e 2 ind. il 07/01/1998 alla foce del fiume Ombrone (PG; GA e PG). Il recente svernamento nel Mediterraneo risulta forse favorito dai cambiamenti climatici in atto (Zenatello et al., 2014). Nel PRM, rilevati a gennaio nel periodo 2013-2017 una media di circa 7 individui svernanti per stagione (fonte: COT, archivio PRM). Ancora recentemente, negli anni 20122015, alcune osservazioni di singoli individui durante tutto il mese di giugno sono difficilmente attribuibili ad estivanti, trattandosi più facilmente di possibili migratori (GA; PG e F. Farsi).

\section{Glareolidae}

\section{Pernice di mare Glareola pratincola $\mathrm{M}$ irr}

In Europa, è specie nidificante in decremento (Staneva $\&$ Burfield, 2017). Durante le migrazioni viene rilevata nelle zone umide costiere con vegetazione emergente e galleggiante, mentre in periodo riproduttivo frequenta $\mathrm{i}$ margini e l'interno delle zone umide salmastre, i campi lavorati di fresco e varie coltivazioni specialmente a sviluppo tardivo (Brichetti \& Fracasso, 2004). Nel PRM, le segnalazioni decorrono verosimilmente dal maggio 1982 nella Palude della Trappola (Romè, 1983b). Più facilmente, la specie durante le migrazioni compare per pochi giorni risultando quindi tra quelle che maggiormente potrebbero aver risentito nel passato di una valutazione fenologica errata per via di uno sforzo di copertura nel rilevamento non sempre adeguato. Successivamente, con inizio dagli anni '90 del secolo scorso, le indicazioni di presenza si riferiscono unicamente alla foce del fiume Ombrone: 1 ind. il 12/07/1992 (PG); 1 ind. il 03/05/1995 (S. Celletti). Monti (2012a) riferisce di osservazioni ad aprile-maggio e luglio durante una indagine sull'avifauna di quell'area negli anni 2008-2010. Segnalazioni più recenti: 2 ind. il 24/04/2012 alla foce del fiume Ombrone (PG); 2 ind. il 01/05/2016 (A. Picciau e D. Giunchi in GOM-List). Interessante concentrazione per l'area: 5 ind. il 15/05/2009, sempre alla foce del fiume Ombrone (GA).

\section{Laridae}

Gabbiano tridattilo Rissa tridactyla $\mathrm{M}$ reg ?, W irr?

In Europa, è specie nidificante in decremento (Staneva $\&$ Burfield, 2017). Nei periodi di migrazione e svernamento frequenta aree pelagiche; occasionalmente rilevato lungo tratti di costa e in aree portuali (Brichetti \& Fracasso, 2006). I dati raccolti lungo il PRM, sino ad un miglio circa dalla riva, sono quindi limitati in ragione dell'ardua rilevabilità della specie. Recenti osservazioni invernali: 1 immaturo il 07/01/2013 sul mare nei pressi del porto di Talamone (GA); 1 ind. il 13/12/2015 lungo la costa dei Monti dell'Uccellina (GA).

\section{Gabbiano roseo Chroicocephalus genei $\mathrm{M}$ irr}

In Europa, è specie nidificante in decremento (Staneva $\&$ Burfield, 2017). In migrazione viene rilevato più facilmente in zone umide salmastre costiere e alle foci di fiumi (Brichetti \& Fracasso, 2006). Nel PRM, compare irregolarmente, unicamente con singoli individui alla foce del fiume Ombrone: 1 ind. il 28/05/1998 (PG); 1 ind. il 29/04/2009 (GA); 1 ind. il 21/08/2016 (ML su Ornitho. it).

\section{Gabbiano comune Chroicocephalus ridibundus $\mathrm{M}$ reg, W reg \\ In Europa, è specie nidificante con trend fluttuan-} te (Staneva \& Burfield, 2017). Durante le migrazioni e lo svernamento frequenta zone umide costiere e dell'interno, di origine naturale o artificiale, con acqua salata o dolce, e con spostamenti quotidiani dai siti di riposo notturno verso quelle zone di alimentazione poste nell'interno (principalmente coltivi in lavorazione, discariche) e viceversa (Brichetti \& Fracasso, 2006). La Macrozona "Maremma Grossetana" è un sito di importanza regionale per lo svernamento della specie (Arcamone et al., 2007). Nel PRM, è specie rilevata durante tutto l'anno per quanto normalmente con contingenti numericamente ridotti a meno di 10 individui tra maggio ed inizio luglio, ma senza indizi di riproduzione. A dicembre segnalate alla foce del fiume Ombrone punte di circa 300 individui negli inverni 1991/92-1993/94 (Giovacchini, 1995h) e, ancora in inverno, 350 ind. il 14/12/1998 alla foce del fiume Ombrone (PG). Evidente il calo dei contingenti svernanti in un ven- 
tennio: raffrontando i dati raccolti nei periodi 1994-1997 e 2014-2017 la media per stagione a gennaio è passata da 164 a 19 individui (fonte: COT, archivio PRM). Individuo inanellato: uno trovato morto ad Alberese, Grosseto, il 14/02/1965, era stato inanellato a Hauho Jokijärvi, Finlandia, il 26/06/1964 (Moltoni, 1973).

Gabbianello Hydrocoloeus minutus M irr, W irr

In Europa, è nidificante in decremento (Staneva \& Burfield, 2017). Nei periodi delle migrazioni e svernamento viene osservato nelle acque marine aperte, zone umide costiere e presso le foci fluviali, con frequentazione dei bacini interni durante i movimenti stagionali; segnalazioni di presenze estive (Brichetti \& Fracasso, 2006). Nel PRM, la specie è rinvenuta occasionalmente alla foce del fiume Ombrone e lungo la costa nord dell'area protetta. Osservazioni forse limitate nel numero dal momento che frequenta più comunemente le acque marine aperte, con le conseguenti difficoltà indotte da una più ardua osservabilità dalla riva, più battuta dai rilevatori. In migrazione: 1 ind. il 25/07/1999 alla foce del fiume Ombrone (B. Parisotto e PG); 1 ind. il 24/04/2002 alla foce del fiume Ombrone (GA e PG). Presenza invernale: 1 ind. il 15/12/1995 vicino alla linea di costa a Principina Mare (PG). Presenza estiva: circa 10 immaturi il 17/06/1987 nella Palude della Trappola (N. Baccetti in Arcamone \& Tellini, 1988). Alcuni individui lungo la costa della Trappola il 20/02/1971 e rinvenimento di un individuo morto sulla spiaggia ( $\mathrm{Di}$ Carlo, 1981).

\section{Gabbiano sghignazzante Leucophaeus atricilla A-1}

Accidentale. 1 adulto e 1 ind. del primo anno il 08/04/2004 alla foce del fiume Ombrone (L. Vanni). Segnalazione omologata dalla COI (Brichetti \& Occhiato, 2005).

Gabbiano corso Ichthyaetus audouinii $\mathrm{M}$ reg, W irr

In Europa, è specie nidificante con trend discontinuo (Staneva \& Burfield, 2017). Frequenta coste marine e isole dove nidifica su scogliere pietrose con copertura erbacea moderata; fuori da tale periodo si rinviene sempre su scogliere pietrose, svernando preferibilmente in mare aperto (Brichetti \& Fracasso, 2006). Nidificante negli anni all'Isola del Giglio (Lambertini, 1997). Nel PRM, le osservazioni fanno principalmente riferimento alle falesie dei Monti dell'Uccellina dove viene rilevato occasionalmente con 1-3 individui al massimo. La specie frequenta per finalità trofiche $\mathrm{i}$ tratti costieri nei pressi di Capo d'Uomo, Le Cannelle e Punta del Corvo, così come l'area immediatamente antistante al porto di Talamone: ad es. 1 adulto il 27/05/1998 alle Cannelle (GA); 2 ind. il 12/07/2003 a Capo d'Uomo (GA); 1 in volo il 06/02/2013 nei pressi della Rocca di Talamone (A. Quaglierini in EBN-Italia); 1 ind. il 13/11/2015 a Punta del Corvo (GA). Nell'area protetta viene anche rinvenuto a nord: 2 ind. il 23/06/1998 alla foce del fiume Ombrone (GA); 2 ind. il 28/03/2009 a Marina di Alberese (M. Dragonetti e F. Corsi). Presenze invernali irregolari: 1 ind. il 08/12/1997 alla foce del fiume Ombrone (GA); 2 ind. al censimento di gennaio 2001 nella Palude della Trappola (Arcamone et al., 2007).
Gabbiano corallino Ichthyaetus melanocephalus M reg, W irr

In Europa, è nidificante in decremento (Staneva \& Burfield, 2017). In periodo extra riproduttivo frequenta acque marine e litorali, anche con copertura vegetale bassa (Brichetti \& Fracasso, 2006). Nel PRM, presenze legate prevalentemente alla sola zona della foce del fiume Ombrone e al tratto antistante di mare aperto. Osservazioni localmente incrementate dall'inizio di questo secolo sebbene numericamente scarse, specialmente se rapportate al panorama regionale in inverno (Arcamone et al., 2007). Nel corso dell'anno concentrazioni di gruppi composti, nella normalità dei casi, da un massimo di circa dieci individui: 8 ind. il 28/03/2009 sul mare a Marina di Alberese (M. Dragonetti e F. Corsi). Raro svernante, anche recentemente: 1 ind. il 03/01/2016 alla foce del fiume Ombrone (A. Galimberti su Ornitho.it). In periodo postriproduttivo: 1 ind. il 18/07/2000 sul mare alle Cannelle di Talamone (GA); 6 ind. il 10/07/2018 alla foce del fiume Ombrone (PG). Concentrazioni inusuali per l'area: 650 ind. il 21/03/2004 alla foce del fiume Ombrone (R. Gildi in EBN-Italia); almeno 30 ind. il 29/03/2006 sul mare verso Cala Francese (A. Delle Monache su Ornitho.it).

\section{Gavina Larus canus $\mathrm{M}$ irr, W irr}

In Europa, è specie nidificante in decremento (Staneva $\&$ Burfield, 2017). Durante le migrazioni e in inverno compare lungo i litorali costieri e nelle zone interne, frequentando ad esempio lagune, foci fluviali, laghi, torbiere e fiumi (Brichetti \& Fracasso, 2006). Nel PRM, compare con pochi individui in migrazione, che risultano spesso difficili da censire per via dell'abitudine ad unirsi a stormi numerosi di Larus michahellis. Nell'area protetta è rilevata occasionalmente in inverno con numeri solo di poco superiori all'unità: 2 ind. il 02/01/2000 alla foce del fiume Ombrone (M. Lausetti). Dati recenti riferiti alle migrazioni: 1 ind. il 25/11/2005 alla foce del fiume Ombrone (PG); 1 ind. il 24/04/2013 alla foce del fiume Ombrone (G. Cazzola su Ornitho.it); 1 ind. il 13/03/2015 alla foce del fiume Ombrone (GA); 1 ind. il 25/02/2016 alla foce del fiume Ombrone (PG).

\section{Gabbiano reale nordico Larus argentatus A-1}

Accidentale. Specie non rinvenuta precedentemente (Giovacchini, 2006) e solo recentemente distinta da Larus michahellis. A complicare le cose, sussistono difficoltà nel riconoscimento sul campo, specialmente degli esemplari immaturi (Arcamone et al., 2007). 1 ind. il 28/12/2001 alla foce del fiume Ombrone (P. Marotto e L. Vanni in Arcamone \& Puglisi, 2006).

Gabbiano reale Larus michahellis $\mathrm{M}$ reg, B, W reg In Europa, è nidificante in incremento (Staneva \& Burfield, 2017). Nidifica generalmente sino a $500 \mathrm{~m}$ s.l.m., prevalentemente sulle pareti rocciose quali falesie, scogliere e isolotti, in zone umide costiere, risultando in espansione nei centri urbani; fuori dal periodo della riproduzione si rinviene facilmente lungo i litorali marini e nelle zone umide dell'interno, alimentandosi comunemente nelle discariche di rifiuti urbani (Brichetti \& Fracasso, 2006). In Toscana, in inverno, $i$ contingenti risultano concentrati lungo costa 
per quanto la specie risulti ampiamente distribuita anche nell'interno; la Macrozona "Maremma Grossetana" è considerata sito di importanza regionale per la specie (Arcamone et al., 2007). Nel PRM, viene rinvenuto comunemente sul mare da Principina Mare a Talamone, più facilmente nella zona della foce del fiume Ombrone e negli specchi lacustri della Palude della Trappola. Una frazione della popolazione potrebbe essere costituita da individui sedentari. Nel periodo 2013-2017 la media a gennaio degli svernanti nell'area protetta è di circa 205 individui (fonte: COT, archivio PRM). Nel corso dell'anno, movimenti pendolari giornalieri di più individui vengono registrati regolarmente anche in questo ambito, con rientri serali verso i dormitori posti lungo costa o sulle isole. In tal senso è nota anche l'utilizzazione di un ampio tratto di litorale sabbioso a sud di Collelungo (Giovacchini \& Celletti, 1995). La nidificazione nell'area protetta è stata accertata per la prima volta nel 1993 sulle falesie dei Monti dell'Uccellina, in località Porticciolo (GA). In quel contesto, nel 1998 censite 23 coppie (GA), poi 15-20 nel 2005 (GA; Franceschi, 2012). Accertata la nidificazione di 1 coppia nella Palude della Trappola: 2 adulti con 3 pulli il 03/06/2012 su una struttura galleggiante in legno posta in un chiaro palustre nei pressi della Casetta Ponticelli (PG e F. Farsi). Stimabile una popolazione minima di 10-11 coppie nidificanti.

\section{Zafferano Larus fuscus M irr, W irr}

In Europa, è specie nidificante in incremento (Staneva $\&$ Burfield, 2017). Durante le migrazioni ed in inverno compare lungo i litorali costieri, frequentando foci fluviali, aree portuali e discariche, così come laghi e fiumi, più spesso in associazione con Larus michahellis (Brichetti \& Fracasso, 2006). Nel PRM, contattato quasi sempre con singoli individui, più facilmente durante le migrazioni alla foce del fiume Ombrone, occasionalmente alle Macchiozze così come al Lasco di Alberese. La specie è infatti rinvenuta raramente in inverno, generalmente con singole presenze, ad es. 1 ind. il 19/01/1994 alla foce del fiume Ombrone (PG); 2 ind. il 06/12/1998 in volo sul fiume Ombrone (PG e SL); 1 ind. il 13/01/2013 alla foce del fiume Ombrone (fonte: COT; archivio PRM). Migrazione primaverile verosimilmente più evidente, con il maggior numero di osservazioni registrate ad aprile. Tra le più recenti: 1 ind. il 17/04/2006 alla foce del fiume Ombrone (PG); 1 ind. il 14/09/2012 al Lasco di Alberese insieme a numerosi Larus michahellis (F. Pezzo).

\section{Sterna zampenere Gelochelidon nilotica $\mathrm{M}$ irr?}

In Europa, è specie nidificante in incremento (Staneva $\&$ Burfield, 2017). Nei periodi di migrazione viene osservata in mare ed in ambienti salmastri costieri; raramente in acque dolci e in campi allagati (Brichetti \& Fracasso, 2006). Nel PRM, è rinvenuta unicamente alla foce del fiume Ombrone, registrando oltretutto limitate presenze in periodo estivo. Tra le segnalazioni più interessanti in periodo tardo primaverile-estivo: 6 ind. il 21/05/1986 (PG); 6 ind. il 02/07/1989 (A. Rivola, D. Occhiato, F. Pieri e G. Scoccianti in Arcamone \& Tellini, 1991-1992); 3 ind. il 04/06/2000 (GA e R. Ferrari); 5 ind. il 23/07/2009 (A. Madella in EBNItalia); 4 ind. il 12/07/2010 (PG); 2 ind. il 27/04/2014 (F. Blangetti su Ornitho.it); 8 ind. il 15/07/2018 (PG).
Sterna maggiore Hydroprogne caspia $\mathrm{M}$ irr, W irr

In Europa, è specie nidificante in incremento (Staneva $\&$ Burfield, 2017). Durante le migrazioni e nel periodo invernale compare più facilmente lungo le coste marine con litorali sabbiosi, in ambienti salmastri costieri, lagune e foci fluviali (Brichetti \& Fracasso, 2006). Nel PRM, le presenze sono riconducibili ad individui osservati prevalentemente durante le migrazioni alla Palude della Trappola, alla foce del fiume Ombrone e lungo i limitati tratti costieri dei Monti dell'Uccellina posti nei pressi delle Cannelle. In tal senso si segnalano, ad es. 1 ind. il 16/09/1995 alla foce del fiume Ombrone (PG); 3 ind. il 17/11/2001 sul mare alle Cannelle di Talamone (GA); 3 ind. il 28/03/2012 alla foce del fiume Ombrone (C. Martelli in GOM-List). Presenze estive: ad es. 2 ind. almeno dal 12 al 17/06/1990 alla foce del fiume Ombrone (P. Politi e L. Becherucci in Arcamone \& Barbagli, 1995-1996); 3 ind. il 29 e 30/06/1993 alla Palude della Trappola (GA); 1 ind. il 04/07/2011 alla foce del fiume Ombrone (M. Biondi). Segnalazione invernale: 1 ind. il 16/12/2003 sul mare alle Cannelle di Talamone (GA).

\section{Beccapesci Thalasseus sandvicensis $\mathrm{M}$ reg, W irr}

In Europa, è specie nidificante con trend discontinuo (Staneva \& Burfield, 2017). Nei periodi delle migrazioni e dello svernamento frequenta più facilmente litorali marini con coste sabbiose e limitrofe zone umide, specialmente ove siano presenti acque limpide e ricche di pesci (Brichetti \& Fracasso, 2006). Nel PRM, presenze non regolari e numericamente fluttuanti nel periodo invernale, più recentemente con punte di 30 individui al censimento di gennaio 2013 alla foce del fiume Ombrone (fonte: COT, archivio PRM). Sempre per la stessa area, interessanti concentrazioni anche nel recente passato: sino a 60 individui nell'inverno 1992-93 (Anselmi, 1995b); 40 ind. il 08/12/1997 (PG); 21 ind. il 24/12/1998 (PG); almeno 100 ind. il 04/01/2002 (S. Celletti e R. Papi). Ugualmente, per la migrazione post-riproduttiva, maggiore accentuazione dei movimenti in vicinanza della foce fluviale, a volte con interessanti concentrazioni per l'area protetta, ad es. 48 ind. il 04/11/1994 (PG); 67 ind. il 21/10/2002 (PG). Limitate segnalazioni da aree immediatamente esterne al PRM, tra le più recenti: 3 ind. il 01/01/2016 nel golfo di Talamone (PG). Presenze occasionali in periodo estivo che, in alcune circostanze, forse non sono ancora imputabili a spostamenti migratori: 4 ind. il 03/07/1995 alla foce del fiume Ombrone (PG); 12 ind. il 16/08/2001 alla foce del fiume Ombrone (PG); 3 ind. il 09/07/2012 alla foce del fiume Ombrone (S. Bassi su Ornitho.it).

\section{Fraticello Sternula albifrons M irr?}

In Europa, è specie nidificante con trend incerto (Staneva \& Burfield, 2017). Durante le migrazioni frequenta acque marine costiere, foci fluviali e zone umide circostanti (Brichetti \& Fracasso, 2006). Nel PRM, osservazioni numericamente limitate e riconducibili, in molti casi, a movimenti migratori registrati alla sola foce del fiume Ombrone: 2 ind. il 03/05/1994 (GA); 6 ind. il 14/05/1998 (PG); 8 ind. il 26/04/1999 (PG). Presenze riferite alla migrazione post-riproduttiva: 7 ind. il 12/07/2003 lungo la costa dei Monti dell'Uccellina (GA); 2 ind. il 07/07/2011 
alla foce del fiume Ombrone (M. Biondi). Dato pregresso: 45 ind. il 11/05/1969 nella zona del San Carlo-Trappola (Castellani, 1970).

\section{Sterna comune Sterna hirundo M reg, W irr}

In Europa, è specie nidificante in incremento (Staneva $\&$ Burfield, 2017). Durante le migrazioni frequenta preferibilmente corsi d'acqua dolce con ampi sabbioni e ghiareti, foci fluviali, lagune e zone umide circostanti (Brichetti \& Fracasso, 2006). Nel PRM, osservazioni regolari negli anni tra la foce del fiume Ombrone, Le Macchiozze e la Palude della Trappola, con una maggiore evidenza dei movimenti pre-riproduttivi per quanto generalmente limitati nella consistenza dei contingenti (più facilmente 1-3 individui): tra i più recenti, ad es. 1 ind. il 11/04/2015 alle Macchiozze (PG e M. Colli). Si aggiungono negli anni occasionali presenze di adulti in periodo estivo, ad es. 1 ind. il 23/06/1995 alla foce del fiume Ombrone (PG); 4 ind. il 08/07/2017 alla foce del fiume Ombrone (PG). Individui in dispersione già dopo metà luglio: 6 adulti ed almeno 3 giovani da poco involati il 18/07/2012 alla foce del fiume Ombrone (C. Martelli in GOM-List). Presenza invernale: 1 ind. dicembre 1988 alla foce del fiume Ombrone (Giovacchini, 1995i). Dato pregresso: 5 ind. il 11/05/1969 nella zona del San Carlo-Trappola (Castellani, 1970).

\section{Mignattino piombato Chlidonias hybrida $\mathrm{M}$ irr}

In Europa, è specie nidificante in incremento (Staneva $\&$ Burfield, 2017). Durante le migrazioni viene rilevato in zone umide costiere ed interne, naturali o artificiali, con fondali poco profondi, foci fluviali, laghi e risaie (Brichetti \& Fracasso, 2006). Nel PRM, osservazioni irregolari negli anni: 6 ind. il 09/05/1998 alla foce del fiume Ombrone (B. Parisotto); alcune segnalazioni riferite alla migrazione primaverile nel periodo 2008-2010 alla foce del fiume Ombrone (Monti, 2012a).

\section{Mignattino alibianche Chlidonias leucopterus M irr}

In Europa, è specie nidificante con trend discontinuo (Staneva \& Burfield, 2017). Nei periodi delle migrazioni frequenta zone umide interne senza disdegnare complessi lacustri di elevate dimensioni e lagune costiere, comunque con acque poco profonde (Brichetti \& Fracasso, 2006). Nel PRM, presenze irregolari negli anni, per quanto sembri verosimilmente più frequente rispetto a Chlidonias hybrida, ad es. 2 ind. il 28/04/1988 alle Macchiozze (E. Arcamone in Arcamone \& Tellini, 1991-1992); 1 ind. il 29/04/1991 alla foce del fiume Ombrone (E. Arcamone in Arcamone \& Barbagli, 1995-1996); 1 ind. il 02/05/2011 alla foce del fiume Ombrone (GA); 1 ind. il 25/09/2014 alla foce del fiume Ombrone (L. Nigro su Ornitho.it).

\section{Mignattino comune Chlidonias niger $\mathrm{M}$ reg, W irr}

In Europa, è specie nidificante con trend sconosciuto (Staneva \& Burfield, 2017). Nei periodi delle migrazioni frequenta complessi lacustri e lagune costiere, foci fluviali, zone umide interne, così come canali, fiumi a corso lento e saline (Brichetti \& Fracasso, 2006). Nel PRM, segnalazioni regolari negli anni alla foce del fiume Ombrone, specialmente con la migrazione pre-nuziale che, per quanto limitata circa al periodo metà aprile-metà maggio, assume per l'area protetta connotati di maggiore rilevanza a volte con elevate concentrazioni numeriche, ad es. 90 ind. il 11/05/1993 alla foce del fiume Ombrone (PG); almeno 200 ind. il 12/05/1998 alla foce del fiume Ombrone (PG). Altrove osservazioni occasionali: 1 ind. il 23/04/1991 nella Palude della Trappola (PG); 2 ind. il 17/05/1992 nelle Risaie di Principina, immediatamente fuori dal PRM (PG); 2 ind. il 06/05/1995 alla Macchiozze (PG). Presenza invernale: 1 ind. il 16/01/1988 alla foce del fiume Ombrone (F. Cianchi \& A. Massi in Brichetti \& Toso, 1989).

\section{Stercorariidae}

Stercorario mezzano Stercorarius pomarinus M irr, W irr

In Europa, è specie nidificante con trend sconosciuto (Staneva \& Burfield, 2017). Durante le migrazioni e lo svernamento appare legata al mare aperto, spingendosi tuttavia sotto costa dove viene rilevato anche in corrispondenza delle foci fluviali (Brichetti \& Fracasso, 2006). Nel PRM, le segnalazioni decorrono verosimilmente dalla fine dello scorso secolo per quanto possa risultare poco definibile la reale frequenza della specie in questo tratto di costa marina: 1 ind. il 21/02/1999 a Marina di Alberese (M. Lausetti). Successive osservazioni riferite a singoli individui alla foce del fiume Ombrone: 1 ind. il 07/10/2000 (E. Occhiato in Arcamone \& Puglisi, 2006); 1 ind. il 02/04/2002 (P. Marotto in Arcamone \& Puglisi, 2006). Presenza invernale: 1 ind. in fase scura il 13/12/2003 a Principina Mare (M. Fedi in EBN-Italia).

\section{Labbo Stercorarius parasiticus $\mathrm{M}$ irr}

In Europa, è specie nidificante con trend sconosciuto (Staneva \& Burfield, 2017). Con le migrazioni e lo svernamento frequenta preferibilmente acque marine aperte $\mathrm{e}$ costiere ma anche grandi laghi e fiumi dell'interno (Brichetti \& Fracasso, 2006). 1 ind. il 23/03/1998 ad un miglio nautico circa dalla costa a Marina di Alberese (Giovacchini, 1999), ma sicuramente poco definibile la reale frequenza della specie in questo tratto di costa marina. Successivamente a questo dato, segnalazioni irregolari di singoli individui in migrazione tra gli anni 2007 e il 2010. La più recente: 1 ind. il 27/03/2010 a Marina di Alberese (F. Corsi e M. Dragonetti).

\section{COLUMBIFORMES}

\section{Columbidae}

\section{Piccione di città Columba livia [var. domestica] SB}

Molto comune, principalmente presso edifici rurali, nelle frazioni al confine del PRM, così come nelle aree coltivate dove viene rilevato durante tutto l'anno. Si segnala la presenza di due colonie fuori da un contesto tipicamente antropizzato: a Torre Bassa, in convivenza con Coloeus monedula e all'abbazia di San Rabano, quest'ultima colonizzata all'inizio degli anni '90 del secolo scorso (PG, GA). 
Colombella Columba oenas $\mathrm{M}$ reg, $\mathrm{W}$

In Europa, è specie nidificante in incremento (Staneva \& Burfield, 2017). Nei periodi di migrazione e svernamento viene generalmente osservata in ambienti con boschi e boschetti planiziali, ricchi di piante arboree con cavità e circostanti spazi aperti dove alimentarsi (Brichetti \& Fracasso, 2006). Nel PRM, presenze regolari da ottobre sino a febbraio, specialmente nell'area compresa tra il Lasco di Alberese e le Tenute di Torre Trappola e di San Carlo ove, ad esempio, si rinvengono individui nelle stoppie di riso, nei campi lavorati e nei seminativi. 20 ind. il 14/01/1979 nella zona del San Carlo-Trappola (C. Castellani). Si registra un evidente aumento dei contingenti in transito e svernanti, a cominciare dall'anno 2015. Verosimilmente risulta oggi più raro osservare, rispetto per esempio agli anni ' 90 del secolo scorso, gruppi in spostamento o in alimentazione aggregati a Columba palumbus (Fig. 39). Dormitori monospecifici: nell'inverno 2017/18 almeno nella zona del Lasco di Alberese (M. Dragonetti e C. Martelli). Tra le osservazioni più recenti: oltre 500 ind. il 18/11/2015 a Pian di Barca, Alberese (PG); 120 ind. il 20/11/2016 alla Trappola nelle stoppie delle risaie (PG e A. Picciau); circa 550 ind. il 17/12/2017 complessivamente nella zona compresa tra il Canale Essiccatore e i Magazzini di Alberese (PG). Quest'ultimo rappresenta in assoluto il dato più elevato registrato nell'area protetta. Distribuzione e consistenza massima dei contingenti in periodo autunno-invernale raffigurati in Fig. 40.

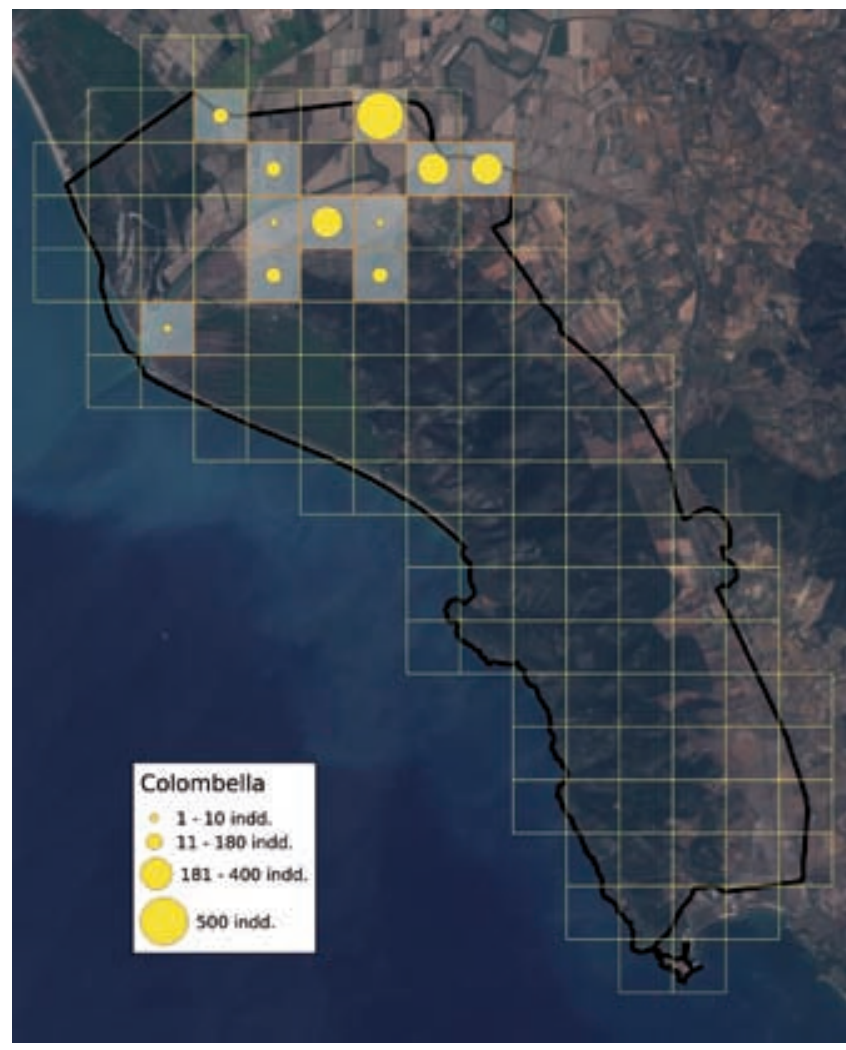

Fig. 40 - Siti di presenza della colombella in periodo autunno-invernale. / Sites of presence of Stock Dove in autumn-winter season (19952017).

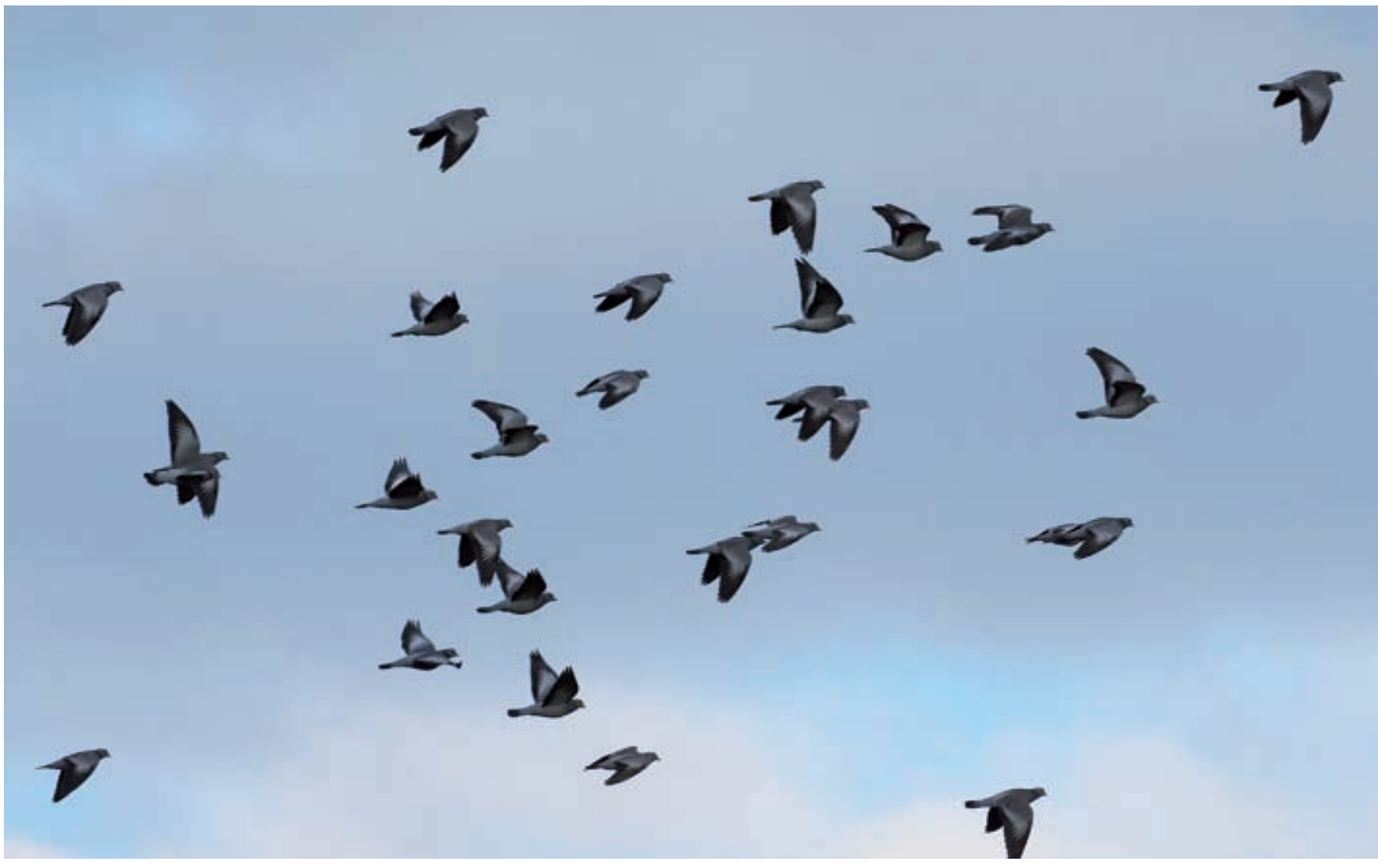

Fig. 39 - Colombelle vicino a Torre Trappola. / Stock Doves near at Torre Trappola (Foto / Photo Stefano Laurenti). 
Colombaccio Columba palumbus SB, M reg, W reg

In Europa, è specie nidificante in incremento (Staneva $\&$ Burfield, 2017). Si riproduce in aree boscate di diversa composizione ed estensione, intercalate da zone aperte, più facilmente sino a circa $1000 \mathrm{~m}$ s.l.m., mentre durante lo svernamento frequenta preferibilmente zone pianeggianti, costiere e collinari, specialmente con formazioni vegetali termofile, affiancate spesso a seminativi o stoppie (Brichetti \& Fracasso, 2006). Nel PRM, viene rilevato diffusamente durante tutto l'anno con una frazione della popolazione che verosimilmente è costituita da individui sedentari; a quelli presenti nel territorio dell'area protetta si assommano esemplari e gruppi in migrazione o in spostamento verso questa importante area di alimentazione. Gli ambiti riproduttivi sono ambienti forestali maturi caratterizzati da Pinus pinea, Quercus ilex e Quercus pubescens, per quanto non vengono disdegnate anche le formazioni ripariali con Populus sp. e i boschi misti anche di limitata estensione. Specialmente in autunno, da ottobre in poi, osservati grossi raggruppamenti nei seminativi e nei pascoli, più raramente con Columba oenas, così come dormitori di alcune migliaia di individui, specialmente in aree indisturbate, ad es. almeno 3000 ind. il 21/11/1995 nei boschi del Collecchio ed altrettanti in quelli del Condotto, Alberese, nell'inverno 2015/16 (GA e PG). Tale comportamento, per quanto apparentemente più raro negli ultimi dieci anni circa, verosimilmente a causa anche della minore produzione di ghiande, nei mesi invernali riguarda specialmente i boschi monospecifici con Quercus ilex (Corsi \& Marcucci, 1995). Prime indicazioni di nidificazione: almeno 1 ind. il 01/06/1992 in località Collecchio, Magliano in Toscana; 1 ind. il 15/05/1994 in località Valentina, Talamone (PG); 1 ind. il 16/06/1997 a Fonte Lepre, Talamone (GA). Stimabile una popolazione minima di 150 coppie nidificanti.

\section{Tortora selvatica Streptopelia turtur M reg, B}

In Europa, è nidificante in decremento (Staneva \& Burfield, 2017). Nidifica in zone boscate aperte, calde e soleggiate, preferendo quelle di pianura e collina coltivate a cereali, più spesso con presenze di siepi, incolti e raccolte d'acqua, normalmente sotto i 300-500 m s.l.m. (Brichetti \& Fracasso, 2006). Nel PRM, è specie rilevata da metà aprile sino ad agosto-inizio settembre, anche a piccoli gruppi, nei mosaici agricoli, siepi e negli incolti soleggiati, spesso in vicinanza di canali e fossi. Più rara nella Pineta Granducale dell'Uccellina ed in quella del San Carlo ove si rinviene specialmente in aree caratterizzate da boschi vetusti con sottobosco. Stimabile una popolazione minima di 50 coppie nidificanti. Nelle stagioni di nidificazione 2000-2004 censiti 53 territori con una frequenza relativa pari a $6,33 \%$.

\section{Tortora dal collare Streptopelia decaocto SB}

In Europa, è nidificante in incremento (Staneva \& Burfield, 2017). Nidifica in aree pianeggianti, caratterizzate da bassa o moderata piovosità, spesso in ambienti rurali così come in zone urbane e nelle loro periferie, con alberi ornamentali, boschi di latifoglie e pinete, anche di limitata estensione, comunque più diffusa sino a 200-300 m s.l.m. (Brichetti \& Fracasso, 2006). Nel PRM, distri- buzione riconducibile principalmente al perimetro orientale dell'area protetta dove più spesso sono rintracciabili insediamenti umani con caseggiati anche sparsi, tipici di ambienti rurali così come di allevamenti zootecnici. Ugualmente rilevata anche nei centri urbani posti ai confini esterni dello stesso PRM (Principina Mare, Alberese, Talamone). Stimabile una popolazione minima di 40 coppie nidificanti. Nelle stagioni di nidificazione 20002004 censiti 28 territori con una frequenza relativa pari a $3,34 \%$.

\section{CUCULIFORMES}

\section{Cuculidae}

Cuculo dal ciuffo Clamator glandarius $\mathrm{M}$ reg, B, W irr In Europa, è specie nidificante in incremento (Staneva \& Burfield, 2017). In Italia, è nidificante "In Pericolo" (Peronace et al., 2012). Parassita di cova, in particolare di Pica pica, frequenta ambienti xerici con pinete litoranee, margini di paludi e prati pascoli cespugliati, sino a 100200 m s.l.m. (Brichetti \& Fracasso, 2006). La prima segnalazione riconducibile all'area dell'attuale PRM risale alla primavera del 1950, con un individuo catturato nella pineta a nord della foce del fiume Ombrone (collezione Federcaccia, Grosseto); nel periodo 1990-2004 la popo-

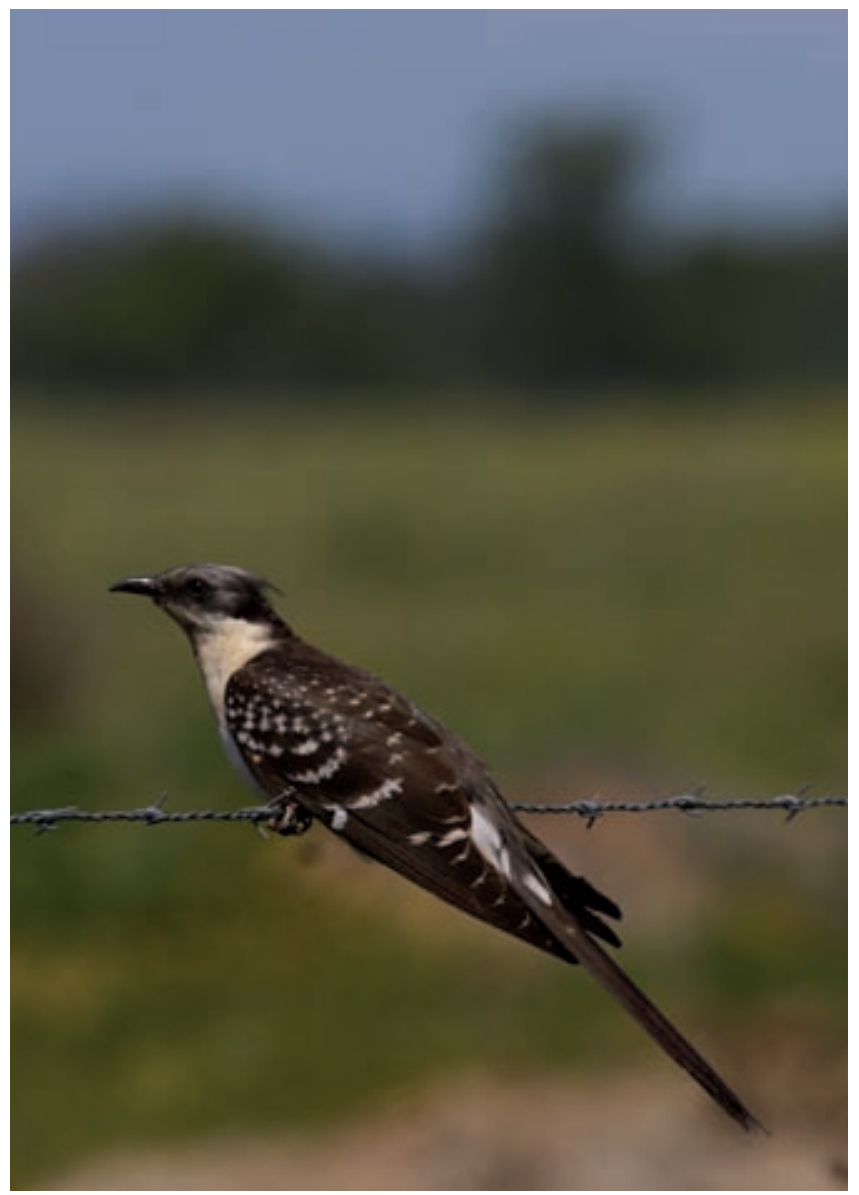

Fig. 41 - Cuculo dal ciuffo alle Macchiozze. / Great Spotted Cuckoo at the Macchiozze (Foto / Photo Giuseppe Anselmi). 
lazione tra Vaccareccia e Principina Mare è stata valutata in 2-3 coppie (Corsi \& Giovacchini, 2001; Giovacchini et al., 2004). Tendenza all'espansione dell'areale, in ragione anche delle favorevoli disponibilità trofiche come ad esempio Thaumetopoea pityocampa, ad es. 1 ind. in richiami il 26/05/2005 in località Valentina di Talamone (PG). Per il periodo 2007-2014, sulla scorta delle osservazioni di adulti e giovani insieme, popolazione ritenuta verosimilmente stabile rispetto al decennio precedente (GA, F. Farsi, F. Corsi). Osservazione invernale: 1 ind. il 10/12/2009 alla foce del fiume Ombrone (F. Monti, com. pers.). Stimabile una popolazione minima di 2 coppie nidificanti (Fig. 41).

\section{Cuculo Cuculus canorus $\mathrm{M}$ reg, B}

In Europa, è nidificante stabile (Staneva \& Burfield, 2017). Parassita di cova, durante la riproduzione frequenta ambienti asciutti boscati, alberati, ma anche zone umide con vasti canneti, specialmente sino a $1400-1500 \mathrm{~m}$ s.l.m. (Brichetti \& Fracasso, 2006). Nel PRM, negli anni, per quanto sicuramente con basse densità, segnalazioni regolari di maschi cantori da metà aprile in poi ai margini del complesso forestale dei Monti dell'Uccellina e della pineta della Tenuta di San Carlo. Scarsa disponibilità di habitat idoneo con vegetazioni elofitica ad esempio nella zona della Palude della Trappola (GA). Stimabile una popolazione minima di 10 maschi cantori.

\section{STRIGIFORMES}

\section{Tytonidae}

Barbagianni Tyto alba SB, M irr?

In Europa, è nidificante con trend discontinuo (Staneva \& Burfield, 2017). Riproduzione in ambito urbano, specialmente in edifici vetusti, così come in cavità di rocce e alberi, ambienti rurali a mosaico con fienili, ruderi, cascinali e manufatti vari, circondati da spazi aperti con coltivazioni erbacee, incolti e spesso da rete viaria, più diffuso sino a 400-500 m s.l.m.; durante l'inverno utilizza sostanzialmente gli stessi ambienti (Brichetti \& Fracasso, 2006). La scarsa contattabilità della specie può comportare una sottostima delle presenze anche nel PRM ove comunque sembra gradire l'esistenza dei querceti mediterranei laddove confinano con spazi aperti: Martelli (1980), in uno studio sullo spettro trofico della specie, ha evidenziato la netta preponderanza dei micromammiferi $(85 \%)$ e degli Uccelli (9\%) nella dieta del barbagianni. Nell'area protetta registrato l'abbandono, nel periodo 2013-2017, di almeno cinque siti "storici" posti in ambienti tra loro diversi e seguiti già tra il 1976 e il 1978 nella zona di Alberese (ad esempio: Spergolaia, Scoglietto) (C. Martelli, ined.). Indicazioni attuali di presenza giungono dalle Tenute di San Carlo e San Mamiliano, forse riconducibili ad individui erratici, come dal settore meridionale di Talamone nella zona della Valentina ove invece è presente almeno 1 coppia, peraltro già nota negli anni ' 90 del secolo scorso (GA; PG), ad es. 1 ind. il 05/05/2014 posato ai margini della strada poderale della Valentina (PG). In un recente passato, osservazioni in periodo autunno-inverna- le nella cava di parete dismessa della Burraia, Alberese: 1 ind. il 10/11 e 01/12/2003 (Giovacchini, 2014). Stimabile una popolazione minima di 1-2 coppie nidificanti.

\section{Strigidae}

Assiolo Otus scops M reg, B, W irr

In Europa, è specie nidificante con trend incerto (Staneva \& Burfield, 2017). Più diffuso sino a 600-700 m s.l.m. e legato ai versanti soleggiati, è nidificante in cavità poste su alberi ai margini di ambienti boscosi, a volte in anfratti rocciosi ed ancora in alberi isolati e parchi cittadini; in inverno è specie difficilmente contattabile per quanto sia possibile rilevarne la presenza ai margini di aree forestali, in ambienti urbani e suburbani, ma sempre con adeguata copertura arborea (Brichetti \& Fracasso, 2006). Nel PRM, l'assiolo è presente lungo la fascia costiera con prevalenza di Pinus pinea, da Principina Mare a Collelungo. I boschi misti delle aree interne posti nelle vicinanze di pascoli e spazi aperti, specialmente da Spolverino sino alla Valentina di Talamone, costituiscono un'altra porzione dell'area protetta dove è possibile rinvenire il rapace notturno. Nella Pineta Granducale dell'Uccellina la specie utilizza per nidificare anche le cassette nido (Pezzo, 2012). L'assiolo è rilevabile al canto più facilmente dalla prima decade di marzo a fine luglio. Si segnala 1 ind. ancora in canto il 31/10/1992 alle Caprarecce, Talamone (PG). Presenze invernali irregolari, unicamente con individui contattati al canto: 1 ind. il 28/01/1997 nei pressi di Alberese (GA); 2 ind. il 26/01/2010 nella pineta a Principina Mare (M. Colli); 1 ind. dicembre 2011 vicino ai pascoli confinanti della Tenuta delle Femminelle, Principina Mare (S. Chechi). Stimabile una popolazione minima di 40 coppie nidificanti.

\section{Gufo reale Bubo bubo A-1}

Accidentale. 1 ind. trovato morto il 19/11/1996 in località Scoglietto, Alberese (Giovacchini, 1999). L'esame effettuato sulla carcassa non ha fornito alcuna indicazione circa una possibile origine aufuga dell'individuo (S. Celletti e GA). Il dato riconferma l'esistenza di movimenti erratici che per la specie potrebbero quindi interessare anche questa parte della Toscana (Rigacci, 1993).

\section{Allocco Strix aluco SB, M irr}

In Europa, è specie nidificante con trend incerto (Staneva \& Burfield, 2017). Rinvenuto in ogni tipo di bosco sia di conifere, di latifoglie o misto ed anche nelle formazioni arboree artificiali, ma comunque con alberi maturi alternati a radure, risulta più diffuso sino a 1000-1200 m s.l.m.; sebbene presentino un'idonea copertura boschiva, alcuni tratti della costa toscana mostrano l'effettiva assenza della specie (Brichetti \& Fracasso, 2006). Nel PRM, difficoltà nel reperire dati particolareggiati sulla distribuzione, con informazioni riferibili quasi unicamente ad individui contattati al canto. Negli anni, presenze stabili, almeno dal 1986, di 1-2 individui nella pineta della Tenuta di San Carlo, così come nelle zone dello Scoglietto, Condotto di Alberese, San Rabano e Torre Bassa: prevale in questo contesto un uso del suolo caratterizzato da boschi e boschetti misti di caducifoglie, spesso associati a cedui 
invecchiati (GA; PG; I. Boschi; V. Ferri; L. Tonini; S. Celletti; A. Gianni; G. F. Martini; C. Martelli; P. Arrigucci; I. Nerozzi). A sud, nelle zone della Valentina di Talamone, Caprarecce e Cannelle, i cedui di sclerofille si impongono sui boschi misti a caducifoglie delineando verosimilmente una modesta presenza del rapace notturno in questa parte dell'area protetta. Stimabile una popolazione minima di 10 coppie nidificanti. Distribuzione meglio precisata rispetto a precedenti lavori (Corsi \& Anselmi, 1991).

\section{Civetta Athene noctua SB}

In Europa, è nidificante stabile (Staneva \& Burfield, 2017). Si riproduce con maggiore frequenza in ambienti aperti purchè accompagnati da alberi sparsi, generalmente sotto i $600 \mathrm{~m}$ s.l.m.; la specie viene anche rilevata nelle aree sottoposte a coltivazioni intensive e nei centri urbani (Brichetti \& Fracasso, 2006). Come in altre regioni, anche in Toscana il rapace notturno appare legato alla presenza di vegetazione arborea, nidificando soprattutto nelle cavità naturali degli alberi (Benucci, 1997). Nel PRM, distribuzione tendenzialmente uniforme e riconducibile principalmente alle zone marginali di aree forestali, in particolare dove la fustaia rappresenta la forma prevalente di coltivazione del bosco. Segnalazioni di individui giungono ugualmente da edifici o manufatti anche in stato di abbandono, nonché dalle adiacenze alle principali sedi stradali, ove paletti o strutture similari sono utilizzati come posatoi notturni durante la caccia. Nella stagione autunnale ed in quella invernale, individui possono essere rinvenuti occasionalmente nelle ore diurne in prossimità delle zone umide (PG). In questo ultimo periodo si è assitito a un probabile incremento della popolazione, forse in massima parte per erratismi verticali. Il quadro che ne emerge porta a definire il rapace notturno come il più comune tra le specie presenti regolarmente durante l'anno. Stimabile una popolazione minima di 30 coppie nidificanti (Fig. 42).

Gufo comune Asio otus M reg, B ?, W irr?

In Europa, è specie nidificante con trend sconosciuto (Staneva \& Burfield, 2017). Legato agli ambienti moderatamente alberati purchè provvisti di sottobosco arbustivo e di zone aperte dove cacciare, più diffuso sino a 1200$1300 \mathrm{~m}$ s.l.m., durante tutto l'anno ben tollera la presenza dell'uomo, anche in contesti decisamente urbani (Brichetti \& Fracasso, 2006). Oggettiva difficoltà a rinvenire il rapace notturno e conseguente limitazione nel delineare un quadro esaustivo della sua distribuzione anche in Toscana (Pezzo, 1997b). In tutti questi anni, nel PRM singoli individui sono contattati principalmente grazie ai richiami spontanei, prevalentemente nell'area compresa tra Scoglietto e Principina Mare, su alberature rade, ai margini di zone umide, alla foce del fiume Ombrone, ma anche nei pressi di cavità naturali, quasi sempre da fine ottobre a tutto aprile, ad es. 1 ind. il 17/12/1993 alla foce del fiume Ombrone (GA); 1 ind. il 28/04/1997 nella pineta di San

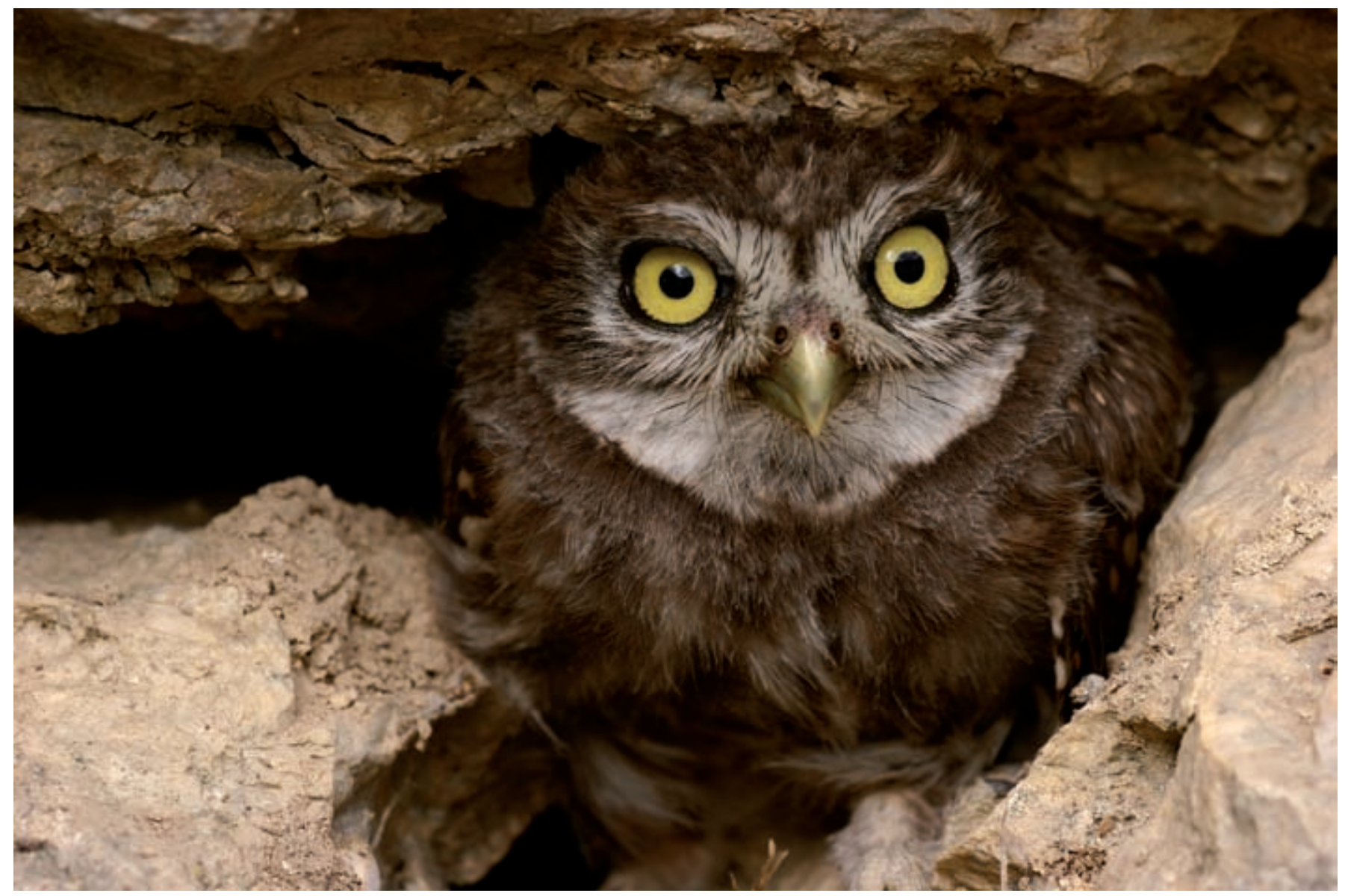

Fig. 42 - Civetta. / Little Owl (Foto / Photo Giuseppe Anselmi). 
Carlo (PG); 1 ind. il 18/03/2002 allo Scoglietto di Alberese (PG); 1 ind. il 14/01/2003 a Vaccareccia (GA); 1 ind. il 10/12/2015 al Chiaro del Porciatti, Palude della Trappola (L. Rigacci). Dormitori invernali occupati irregolarmente: nella stagione 1990/91 sino a 5 ind. nella pineta della Tenuta di San Carlo (GA); nella stagione 1999/2000 con 3 ind. il 26/01/2000 su Quercus ilex lungo la strada per San Rabano (GA e G. F. Martini). Un'unica segnalazione proveniente dalla zona meridionale dell'area protetta: 1 ind. il 18/12/1996 in località Casacce, Talamone (PG). Ritrovamento nel luglio 1987 a Marina di Alberese di un esemplare adulto ferito (Corsi \& Anselmi, 1991). Si segnala inoltre 1 ind. il 08/07/2017 nella pineta a Campo al Pino, Alberese (PG).

\section{Gufo di palude Asio flammeus M irr, W irr}

In Europa, è nidificante con trend fluttuante (Staneva \& Burfield, 2017). Più crepuscolare che notturno, ma attivo anche durante le ore diurne, la specie durante le migrazioni frequenta specialmente spazi aperti o moderatamente cespugliati, estesi coltivi, prati e stadi iniziali di rimboschimenti, mentre in periodo invernale utilizza margini di zone umide costiere (Brichetti \& Fracasso, 2006). Nel PRM, la specie è contattata irregolarmente con singoli individui, per lo più alla foce del fiume Ombrone. Le osservazioni decorrono apparentemente dall'inizio degli anni ' 80 del secolo scorso e fanno riferimento alla migrazione autunnale (Corsi \& Anselmi, 1991). Successivamente, per quanto limitate nel numero, la maggior parte delle segnalazioni risalgono al 1991: 1 ind. il 20 e 21/04/1991 alla foce del fiume Ombrone (D. Occhiato in Arcamone \& Barbagli, 1995-1996); 1 ind. il 20/10/1991 nei pressi della Palude della Trappola (F. Pieri). Occasionale in inverno, anche in contiguità esterna con il PRM: 1 ind. il 13/01/1981 ai margini della Palude della Trappola (M. Pani, ined.); 1 ind. almeno il 01/12/2002 in volo sulla cava dismessa della Voltina (PG e S. Paoloni); 1 ind. il 20/12/2007 alla foce del fiume Ombrone (E. Di Masso in EBN-Italia). Recente segnalazione in periodo primaverile: 1 ind. il 20/03/2018 alla foce del fiume Ombrone (G. Radi in GOM-List).

\section{CAPRIMULGIFORMES}

\section{Caprimulgidae}

\section{Succiacapre Caprimulgus europaeus M reg, B}

In Europa, è nidificante con trend stabile (Staneva \& Burfield, 2017). Durante la nidificazione è frequente rilevarne la presenza in zone asciutte con vegetazione arborea e arbustiva sparsa, più facilmente intervallate da radure, ghiareti, incolti, garighe e calanchi, normalmente sino a 800-1000 m s.l.m. (Brichetti \& Fracasso, 2006). Rinvenuto al canto notturno da fine aprile a luglio, nel PRM è contattabile in particolare ai margini delle fustaie di recente avviamento, nelle garighe ed in altri ambienti xerici posti in corrispondenza di estesi affioramenti rocciosi collinari con vegetazione arbustiva ed arborea discontinua. In tal senso ne viene segnalata la presenza ai margini e lungo la catena dei Monti dell'Uccellina come, ad esempio, in località Scoglietto di Alberese, Cala di Forno, lungo la stra- da del Condotto, a Capo d'Uomo, Talamone, ma anche nelle zone totalmente pianeggianti come quelle ricadenti nella Tenuta di Torre Trappola. Nidificazione accertata: 1 ind. in cova il 02/07/2002 a Cala di Forno (GA); 1 ind. in cova il 24/07/2018 nei pressi della Strada degli Ulivi, Alberese (F. Ferretti). Osservazione autunnale: 1 ind. il 11/11/2001 allo Scoglietto di Alberese (PG). Stimabile una popolazione minima di 25 coppie nidificanti.

\section{APODIFORMES}

\section{Apodidae}

Rondone maggiore Tachymarptis melba $\mathrm{M}$ reg?

In Europa, è nidificante con trend stabile (Staneva \& Burfield, 2017). Si riproduce in zone rupestri costiere e dell'interno, in aree urbane dove preferisce manufatti vari purché elevati in altezza, aree montuose, perilacustri e pedemontane, gole rocciose, calanchi, pareti calcaree strapiombanti con grotte ed anfratti, normalmente sino a 1300-1500 m s.l.m.; movimenti alla ricerca di cibo normalmente lontano dai siti riproduttivi (Brichetti \& Fracasso, 2007). In Toscana, lungo costa, le falesie alte e frastagliate, nonchè le rupi dominanti, per esempio con torri e fari, costituiscono in massima parte gli ambienti prescelti dalla specie (Arcamone \& Roselli, 1997). Nel PRM, segnalazioni irregolari, riferite agli anni 19892013, quasi sempre nei periodi delle migrazioni, tra aprile e metà maggio e nell'ultima decade di luglio-settembre, in particolare riferite alle zone della Trappola, Fattoria Granducale di Alberese, Scoglietto di Alberese, falesie dei Monti dell'Uccellina ed aree di poco esterne come la Rocca di Talamone (B. Parisotto; D. Verducci in GOMList; A. Lamek su Ornitho.it; A. Rivola e P. Politi in Arcamone \& Tellini, 1991-1992; D. Occhiato in Arcamone \& Barbagli, 1995-1996; M. Marcelli in GOM-List). Maggiori concentrazioni, unicamente basate su individui rilevati in volo: 35 ind. il 01/05/1999 in località Punta del Corvo, Talamone (GA e PG); oltre 10 ind. il 12/04/2002 alla Fattoria Granducale di Alberese (PG); almeno 12 ind. il 16/08/2004 tra il cimitero e il campeggio di Talamone, in area immediatamente esterna (M. Dragonetti); 9 ind. il 20/04/2013 nei pressi di Talamone (L. Boscain in EBNItalia). La segnalazione di 1 ind. il 02/07/1989 nei pressi dei Monti dell'Uccellina è più probabilmente attribuibile a spostamenti da un possibile sito riproduttivo (per esempio: Monte Argentario, Isola del Giglio) per finalità trofiche (A. Rivola e D. Occhiato in Arcamone \& Tellini, 1991-1992). Nel passato segnalazioni generiche a riguardo della presenza di una colonia sui Monti dell'Uccellina (Di Carlo, 1981).

\section{Rondone comune Apus apus $\mathrm{M}$ reg, $\mathrm{B}, \mathrm{W}$ irr}

In Europa, è nidificante in decremento (Staneva \& Burfield, 2017). La riproduzione avviene in centri urbani, cascinali e manufatti vari, normalmente sino a 1300-1500 m s.l.m. (Brichetti \& Fracasso, 2007). Nel PRM, limitate presenze di coppie riproduttrici, con un esiguo numero di insediamenti nel contesto proprio dell'area protetta in Alberese (Fattoria Granducale di Alberese, Vaccareccia). 
In massima parte risultano evitate le costruzioni in aree rurali, molto spesso a causa di scelte architettoniche che offrono scarsa attrattività per la specie. La situazione si caratterizza per essere più favorevole in alcuni centri urbani confinanti con il PRM stesso (Alberese, Talamone). Il 10/05/1991 rilevata sulla spiaggia di Marina di Alberese la morte di decine di individui in migrazione, quale conseguenza di un improvviso abbassamento della temperatura (PG). Prima segnalazione di svernamento in Toscana: 1 ind. il 13/01/1996 alla foce del fiume Ombrone (PG in Arcamone \& Brichetti, 1997). Stimabile una popolazione minima di 10 coppie nidificanti.

Rondone pallido Apus pallidus $\mathrm{M}$ irr ?, B irr, W irr

In Europa, è specie nidificante con trend stabile (Staneva $\&$ Burfield, 2017). Si riproduce preferibilmente lungo le coste marine con falesie e scogliere ricche di grotte ed ampie spaccature; presente anche in centri urbani, specialmente costieri, normalmente sino a $300 \mathrm{~m}$ s.l.m. (Brichetti \& Fracasso, 2007). Nel PRM, segnalato come nidificante sui Monti dell'Uccellina almeno sino alla stagione riproduttiva 2006, con una colonia monospecifica stimata al massimo in circa 10 coppie alla Grotta della Casa, Talamone (GA). Di difficile intrepretazione l'osservazione di alcuni individui il 27/07/2011 alla vicina Rocca di Talamone (D. Verducci in GOM-List). Dato pregresso: 1 ind. il 17/08/1983 costa dei Monti dell'Uccellina (A. Rivola in Arcamone \& Tellini, 1985). Osservazione invernale: 2 ind. il 27/12/2010 lungo la costa dei Monti dell'Uccellina (F. Fabbrizzi in GOM-List).

\section{CORACIIFORMES}

\section{Coraciidae}

Ghiandaia marina Coracias garrulus $\mathrm{M}$ reg, B

In Europa, è nidificante in decremento (Staneva \& Burfield, 2017). In Italia, è specie nidificante "Vulnerabile" (Peronace et al., 2012). Riproduzione nelle cavità naturali di alberi maturi o nelle cavità di muri, ponti, pali in cemento e manufatti vari, ma anche in nidi artificiali o scavati da altri uccelli come, ad esempio Picus viridis, comunque non oltre $300 \mathrm{~m}$ s.l.m. (Brichetti \& Fracasso, 2007). Le segnalazioni relative alla parte di territorio oggi tutelato come PRM ne indicano la presenza storica già sul finire dell'Ottocento (Baccetti \& Meschini, 1986). Da oltre trent'anni è specie oggetto di proposte e azioni messe in atto a favore della sua conservazione dopo aver rischiato localmente l'estinzione (Anselmi et al., 1989). Nell'area protetta, infatti, la gestione delle attività forestali e zootecniche nella Zona di Protezione Speciale IT51A0014 "Pineta Granducale dell'Uccellina" assume anche per la specie, in quanto dominante nella comunità nidificante, una significativa rilevanza rispetto ad altri contesti dello stesso comprensorio (Pezzo, 2012). Dati disponibili relativi ai singoli anni 1991 e 2003 nonchè attraverso il monitoraggio della popolazione dal 2007 mostrano una situazione che decorre con 8-10 coppie nel 1991 e giunge ad un aumento marcato sino al 2010, anno in cui è stata accertata la presenza del massimo numero di coppie presenti nel PRM ( $\mathrm{n}=52$ ); successivamente, sino al 2013, la diminuzione registrata sembra aver portato la popolazione ad un effettivo compreso tra 30-40 coppie nidificanti (Corsi \& Anselmi 1994; Giovacchini, 2006; Dottori, 2008; Scoccati, 2014). In questo senso, i nidi artificiali installati negli anni $(\mathrm{n}=47)$ si sono rilevati un efficace strumento di conservazione tale da contribuire all' incremento della importante popolazione del PRM (Pezzo \& Cianchi, 2015) (Fig. 43). La specie è rinvenuta regolarmente in riproduzione anche poco più a nord, sempre in prossimità di spazi aperti: dalla pineta della Tenuta di San Carlo e poi lungo il corso del fiume Ombrone verso la zona della Pineta Granducale dell'Uccellina. Segnalazione di nidificazione in habitat inusuale per l'area protetta: 1 coppia osservata il 01/07/2011 e il 04/07/2011 portare cibo ai nidiacei nelle fessure dei muri dell'ex centro aziendale delle proprietà Vivarelli Colonna, Collecchio (R. Ragazzini). Osservazione più tardiva: 1 ind. il 26/09/2014 lungo le golene del fiume Ombrone (M. Anselmi). Segnalazioni storiche: 1 coppia il 11/05/1969 e 23/06/1974 nella pineta Granducale, Alberese (Castellani, 1970 e ined.). Stimabile una popolazione minima di 13-15 coppie nidificanti.

\section{Alcedinidae}

Martin pescatore Alcedo atthis $\mathrm{M}$ reg, $\mathrm{B}, \mathrm{W}$ reg

In Europa, è nidificante in decremento (Staneva \& Burfield, 2017). Specie presente con coppie isolate: il nido è collocato in una galleria scavata in scarpata prossima all'acqua, normalmente sino a 300 m s.l.m. (Brichetti

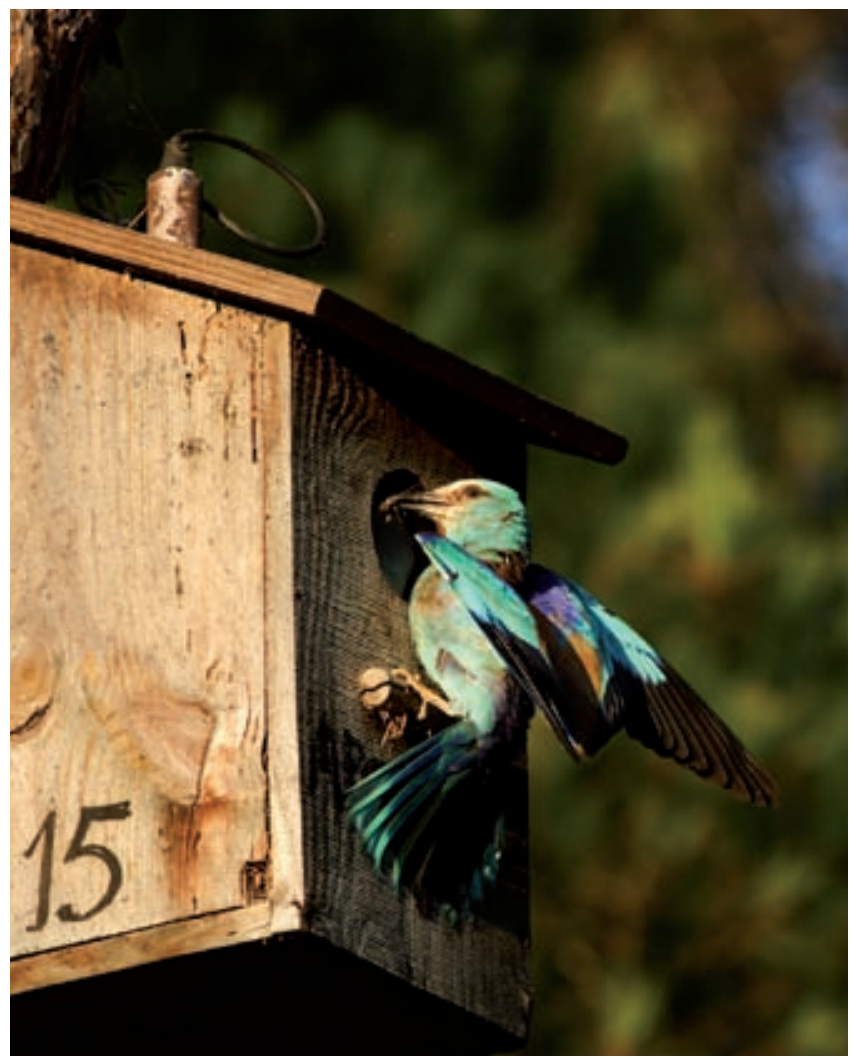

Fig. 43 - Ghiandaia marina nella Pineta Granducale di Alberese. / Roller in the Granducale pinewood of Alberese (Foto / Photo Giuseppe Anselmi). 
$\&$ Fracasso, 2007). Più recentemente, sino alla stagione riproduttiva 2017, 2 coppie nidificano regolarmente nel PRM in aree con pareti nude poste lungo il fiume Ombrone tra Pian di Barca e la foce dello stesso (M. Simoni). Nel 2015, rilevato un probabile sito nei pressi dello Scoglietto di Alberese, con alcune osservazioni raccolte da inizio luglio sino alla conclusione della stagione riproduttiva: 1 ind. il 27/07/2015 vicino al Canale Essiccatore (P. Arrigucci; PG). In autunno-inverno anche il reticolo idrografico della Trappola, sino alla omonima Palude, è stabilmente frequentato da 1-2 individui (GA; M. Dragonetti; PG, M. Brandi, R. Sirna, F. Ferragamo Visconti e G. Visconti). La nidificazione è ritenuta possibile nell'area del Canale di Bonifica di Talamone (GA). In questa zona, singoli individui svernano regolarmente con osservazioni che apparentemente decorrono da oltre trent'anni orsono: 1 ind. il 12/12/1987 (PG). Occasionale altrove: 1 ind. il 25/12/2000 alle Macchiozze (PG). Migrazione in atto: 3 ind. insieme il 22/09/2002 alla foce del fiume Ombrone (PG). Stimabile una popolazione minima di 1-2 coppie nidificanti.

\section{Meropidae}

\section{Gruccione Merops apiaster $\mathrm{M}$ reg, B}

In Europa, è nidificante stabile (Staneva \& Burfield, 2017). Si riproduce in ambienti aperti e xerici, caratterizzati da vegetazione assente o discontinua, e con un substrato prevalentemente sabbioso o misto con argilla, in condizioni idonee per la costruzione del nido, con gallerie scavate specialmente in corrispondenza di scarpate, terreni di riporto e golene fluviali, più diffuso sino a 300 m s.l.m. (Brichetti \& Fracasso, 2007). Dal 2012 circa osservata nel PRM una sensibile diminuzione dei contingenti nidificanti; regolare negli anni la presenza di singole coppie distribuite lungo fossi e canali tra le località Torre Trappola e Collecchio (GA). Da segnalare due vaste aree utilizzate per la riproduzione, sebbene oggi di minore significativà rispetto anche agli anni ' 90 del secolo scorso: la prima lungo il fiume Ombrone, tra Pian di Barca e la foce dello stesso, e in una area limitatamente interna della Trappola caratterizzata da riporti di terreno; la seconda corrispondente al confine sud dell'area protetta tra la Valentina ed il Collettore Occidentale, Talamone. Migrazioni meno evidenti lungo costa rispetto ai settori interni della provincia. Osservazione più tardiva: 1 ind. il 14/10/2003 alla foce del fiume Ombrone (GA e L. Tinti). Stimabile una popolazione minima di 10 coppie nidificanti.

\section{BUCEROTIFORMES}

\section{Upupidae}

Upupa Upupa epops $\mathrm{M}$ reg, B

In Europa, è nidificante stabile (Staneva \& Burfield, 2017). Si riproduce in ambienti aperti assolati di pianura e collina, in filari con boschetti e vecchi alberi meglio se associati a siepi, in manufatti con cavità, parchi di ville patrizie, in vecchi frutteti, oliveti ma anche in aree urbane caratterizzate dalla presenza di caratteristiche ecologiche idonee, più diffusa sino a $800-900 \mathrm{~m}$ s.l.m. (Brichetti \& Fracasso, 2007). Nel PRM, occupati più facilmente i settori di pianura posti ai margini delle pinete ed i coltivi arborei, mentre risultano scarse le indicazioni di riproduzione nei manufatti, in ragione sicuramente della vasta disponibilità di cavità naturali adatte: 1 ind. il 03/06/2000 accede con l'imbeccata in una fessura della Torre Trappola $(\mathrm{PG})$. I primi arrivi nell'area protetta sono più facilmente collocabili nella seconda decade di marzo. Stimabile una popolazione minima di 20-30 coppie nidificanti.

\section{PICIFORMES}

\section{Picidae}

Torcicollo Jynx torquilla M irr ?, B ?, W irr

In Europa, è specie nidificante in incremento (Staneva \& Burfield, 2017). In Italia, è nidificante "In Pericolo" (Peronace et al., 2012). Durante l'anno rilevato più facilmente in vari tipi di ambienti boscati sotto gli 800-900 m s.l.m., preferibilmente aperti con soprassuoli caratterizzati da latifoglie, ma anche in vecchi frutteti, oliveti, parchi, giardini e orti urbani; in inverno, comunque, accentuazione delle osservazioni in aree boscate costiere pianeggianti e di collina così come in ambiente urbano (Brichetti \& Fracasso, 2007). Nel PRM, presenze limitate negli anni e circoscritte ai settori centro settentrionali dell'area protetta. Le osservazioni fanno quasi sempre riferimento a singoli individui, con rare eccezioni: 3 ind. il 25/01/1993 a Principina Mare (PG). In autunno-inverno la specie viene contattata anche in prati pascoli prossimi a zone umide $\mathrm{o}$ alla foce del fiume Ombrone. Tra le osservazioni più recenti: 1 ind. il 27/11/2006 lungo le golene del fiume Ombrone (PG); 1 ind. il 01/05/2010 a Marina di Alberese (M. Fedi su Ornitho.it); 1 ind. il 03/07/2011 nella Pineta di San Carlo (M. Biondi).

Picchio rosso maggiore Dendrocopos major $\mathrm{M}$ irr ?, B, W reg

In Europa, è specie nidificante stabile (Staneva \& Burfield, 2017). Presente in vari tipi di ambienti boscati e alberati, di latifoglie e conifere, purché con alberi morti o deperienti in cui scavare fori di alimentazione e nidi. Comune anche in pioppeti maturi, comunque diffuso dal livello del mare sino ai $1700 \mathrm{~m}$ circa s.l.m. (Brichetti \& Fracasso, 2007). Nel PRM, la specie risulta segnalata per la prima volta in occasione di movimenti erratici registrati nell'inverno 1993/94: 1 ind. in richiami il 19/12/1993 e 30/01/1994 in località Collecchio (GA). Sono verosimilmente due le vie di ingresso della specie nell'area protetta: la prima dal sistema boschivo circostante ai settori centrali del PRM in quanto spazialmente più vicino e simile in fatto di habitat; la seconda attraverso il corridoio ecologico fornito dal corso del fiume Ombrone dove, tra l'altro, si rinvengono maggiormente alberi senescenti o morti. Potrebbero trattarsi delle prime segnalazioni per l'intera pianura grossetana (Tellini Florenzano, 1997a). Negli anni 1997-1998 se ne rileva infatti la presenza in inverno tra Spolverino e Torre Trappola con individui sino alla vicina pineta della Tenuta di San Carlo (GA; PG; L. Minucci e L. 
Buratta). La specie nel 2010 è ancora assente dalla Pineta Granducale dell’Uccellina (Pezzo, 2012). Scarne le segnalazioni in periodo riproduttivo: 1 ind. il 09/05/1999 in località Spolverino (PG); 1 ind. il 23/05/2012 nella Pineta Granducale dell'Uccellina (A. Martelli e C. Martelli in GOM-List). Stimabile una presenza minima di 1 coppia nidificante.

\section{Picchio verde Picus viridis $\mathrm{SB}$}

In Europa, è specie nidificante in incremento (Staneva $\&$ Burfield, 2017). Frequenta zone boscate diversificate, anche di modeste estensioni, pure o miste, comunque ricche di alberi d'alto fusto isolati ed in corrispondenza di spazi aperti, nidificando generalmente sino a 1200-1300 m s.l.m.; sul versante tirrenico predilige pinete litoranee di Pinus pinea (Brichetti \& Fracasso, 2007). Nel PRM, la specie risulta ben distribuita nella fascia a pineta compresa tra Principina Mare e Alberese, lungo il corso del fiume Ombrone e nei settori settentrionali e centrali dei Monti dell'Uccellina con formazioni forestali termofile, occupando anche spazi di modesta estensione (per esempio: Lasco di Alberese). Rilevato quasi sempre con singoli individui. È una delle specie dominanti della comunità nidificante nella Pineta Granducale dell'Uccellina (Pezzo, 2012). Nelle stagioni di nidificazione 2000-2004 censiti 23 territori con una frequenza relativa pari a $2,74 \%$. Stimabile una presenza minima di 50-60 coppie nidificanti.

\section{FALCONIFORMES}

\section{Falconidae}

\section{Grillaio Falco naumanni M irr?}

In Europa, è specie nidificante in incremento (Staneva $\&$ Burfield, 2017). Durante le migrazioni viene osservato in ambienti aperti, caldi e secchi, circondati da aree di alimentazione incolte o coltivate in modo estensivo (Brichetti \& Fracasso, 2003). In Toscana segnalazioni di nidificazione ormai datate, comunque riconducibili al settore costiero meridionale (La Gioia et al., 2017). Nel PRM, è specie segnalata inizialmente da Lovari \& Martelli (1977). È realistico ipotizzare che qualche individuo passi inosservato perché confuso con Falco tinnunculus. Successivamente, le uniche indicazioni raccolte dal 1987 al 2004 riguardano singoli maschi erratici osservati in volo nel mese di aprile del 1989 e 1997, rispettivamente a Spergolaia ed a Principina Mare (GA). Successivamente al 2012, segnalazioni di adulti anche in periodo riproduttivo ed in gruppi: 5 ind. il 01/09/2014 al Salto del Cervo, Alberese (C. Martelli in GOM-List); 1 ind. il 27/05/2015 in volo sulla Palude della Trappola (PG e A. Picciau).

\section{Gheppio Falco tinnunculus SB, M reg, W}

In Europa, è specie nidificante in decremento (Staneva \& Burfield, 2017). Si riproduce più facilmente con coppie isolate, in zone rupestri e forestali aperte, tralicci, edifici, utilizzando anche nidi abbandonati da altri uccelli, più diffuso sino a $2000 \mathrm{~m}$ s.l.m. (Brichetti \& Fracasso, 2003). È tra i più comuni rapaci diurni nidificanti nel
PRM (Petretti, 2008). Una frazione della popolazione è verosimilmente costituita da individui sedentari, con un evidente incremento numerico dei contingenti in inverno, specialmente nei tratti aperti di pianura. Nonostante la natura eclettica nella scelta del sito riproduttivo, il rapace viene più facilmente osservato laddove la pineta cinge da Principina Mare a Collelungo questa parte della fascia costiera. In dettaglio si insedia nelle zone di transizione con Pinus pinea attigue ai prati pascoli, incolti o radure e nel suo settore più interno (per esempio: Serrata dei Tre Pini, Le Grotte, Poste del Granduca). L'utilizzazione delle pareti rocciose dei Monti dell'Uccellina sembrerebbe ridimensionata rispetto al passato (Martelli, 1983). Rinvenuto occupare durante tutto l'anno la cava di parete dismessa della Burraia, Alberese (Giovacchini, 2014). Almeno nei primi anni ' 90 del secolo scorso nidificante anche in manufatti isolati a Cala di Forno ed nelle zone di Fonte Lepre-Poggio alla Murcia, in ambienti caratterizzati da alberature sparse (GA e PG). Nel periodo 19862004 stima di 6-9 coppie presenti, con una densità di 1 coppia/7,7-11,6 km² (Giovacchini, 2006). Stimabile una presenza minima di 5-6 coppie nidificanti.

\section{Falco cuculo Falco vespertinus $\mathrm{M}$ irr}

In Europa, è specie nidificante in decremento (Staneva $\&$ Burfield, 2017). In migrazione è possibile osservarla in aree aperte pianeggianti con filari alberati, ricche di insetti, più facilmente in prossimità di zone umide o canali (Brichetti \& Fracasso, 2003). Nel PRM, è un migratore scarso, quasi sempre solo primaverile (Martelli, 1983). Dal 1990 al 2004 raccolte segnalazioni di presenza per 7 anni. La specie utilizza negli anni, come posatoio, la linea elettrica esistente a ridosso della strada provinciale della Trappola e della Tenuta di San Mamiliano. Molto scarse le segnalazioni provenienti dai settori meridionali dell'area protetta: 1 ind. il 18/03/1998 a Talamone (PG). Tra le più recenti osservazioni: 1 femmina il 31/05/2015 alle Saline di San Paolo, foce del fiume Ombrone (PG); 1 femmina il 22/05/2018 a ridosso della Tenuta di San Mamiliano, lungo la strada provinciale della Trappola (PG).

\section{Falco della regina Falco eleonorae $\mathrm{M}$ reg}

In Europa, è nidificante in incremento (Staneva \& Burfield, 2017). La specie si distingue, in particolare, per alcuni aspetti legati al ritardato calendario riproduttivo, tale da permettere lo sfruttamento delle risorse trofiche indotte dalla migrazione autunnale di piccoli uccelli, eleggendo a colonie alcune zone poste su rocce delle due maggiori isole del Paese ma anche di quelle satelliti (Brichetti \& Fracasso, 2003). Nel PRM, osservato da aprile ad ottobre, generalmente con 1-4 individui, estivanti o migratori, in volo per lo più nelle ore crepuscolari, in un'ampia fascia a Pinus pinea posta nei pressi di Marina di Alberese, insieme a zone aperte che comprendono in massima parte la Palude della Trappola, la foce del fiume Ombrone ed i prati contigui; visitate anche zone rocciose interne. Prevalente la fase di colore chiara $(78,8 \%)$ su un campione di 47 individui osservati con certezza tra il 1994-1996 (Giovacchini \& Celletti, 1997). Il PRM non ospita dunque colonie riproduttive: 1'alimentazione sembra basarsi su Coleotteri ed Odonati. Tra le massime presenze: 12 
ind. il 03/10/1995 complessivamente tra Castel Marino e Scoglietto, Alberese (S. Celletti); 10 ind. il 29/07/1996 a Marina di Alberese (GA e PG). Le osservazioni più recenti indicano contingenti numericamente inferiori, ad es. 2 ind. il 01/09/2014 a Spergolaia, Alberese (C. Martelli in GOM-List); 2 ind. il 04/08/2016 al confine di Principina Mare (PG in GOM-List). A volte osservato cacciare in gruppo con Falco subbuteo, specie con analogo spettro trofico.

\section{Smeriglio Falco columbarius $\mathrm{M}$ reg, W reg}

In Europa, è specie nidificante con trend sconosciuto (Staneva \& Burfield, 2017). Svernante in ambienti erbosi con alberi ed arbusti sparsi (Brichetti \& Fracasso, 2003). Nel PRM, rinvenuto con singoli individui dalla seconda quindicina di ottobre a fine febbraio; occasionali le presenze maggiori: 2 ind. il 14/12/2005 al Lasco di Alberese (PG). Osservazioni sporadiche sino a metà marzo: 1 ind. il 14/03/1996 allo Scoglietto, Alberese (S. Celletti); 1 ind. il 17/03/1999 in località Romitorio, Alberese (PG). Infatti, su un totale di oltre 40 osservazioni raccolte dal 1986 al 2017 solo 5 sono riferite al periodo metà febbraio-marzo, con la migrazione primaverile che potrebbe procedere seguendo rotte differenti da quelle utilizzate per lo svernamento. Nell'area protetta frequenta quasi esclusivamente le zone di pianura e collinari comprese tra Alberese e Principina Mare. Nel passato episodiche segnalazioni dalla zona di Talamone, ad es. 1 ind. il 08/02/1992 (PG). Più recentemente: 1 femmina il 19/01/2016 a Spolverino (PG); 1 maschio il 23/01/2017 nella Palude della Trappola (GA); 1 maschio il 26/12/2017 alle Macchiozze (PG). 1 maschio il 12/12/2007 osservato attaccare 1 maschio di Accipiter nisus, Alberese (PG).

\section{Lodolaio Falco subbuteo M reg, B, W irr}

In Europa, è specie nidificante stabile (Staneva \& Burfield, 2017). Nel periodo riproduttivo è presente generalmente dal livello del mare sino a $700 \mathrm{~m}$ s.l.m. circa, in ambienti caratterizzati da aree boscate intervallate da ampie radure ove caccia le prede costituite soprattutto da Insetti e passeriformi; non costruisce il nido, ma ne utilizza uno abbandonato da corvidi, preferibilmente Pica pica o Corvus cornix (Brichetti \& Fracasso, 2003). Nel PRM, è specie in moderato incremento come nidificante dall'inizio di questo secolo, osservata da maggio a metà ottobre. Negli anni '80 del secolo scorso ritenuto unicamente migratore (Corsi \& Anselmi, 1991). Nel periodo riproduttivo la distribuzione coincide con la parte settentrionale dell'area protetta: dal confine posto a Principina Mare sino a Spolverino-Marina di Alberese, in una vasta zona caratterizzata dal corso del fiume Ombrone, prati pascoli con alberature sparse ed aree boscate (GA; PG; F. Pezzo). Nella Pineta Granducale dell'Uccellina stimata oggi una popolazione di 3-5 coppie nidificanti (Pezzo, 2012). Ulteriori segnalazioni giungono dopo il 2010 dalla località Salto del Cervo, posta poco più a sud dello Scoglietto, Alberese (C. Martelli). Predazione possibile su adulti di Coracias garrulus (F. Pezzo, com. pers.). Accidentale in inverno: 1 ind. il 29/12/1982 nel "Parco dell'Uccellina" (Bonazzi \& Vigarani in Toso, 1983). Nell'area protetta stimabile una popolazione minima di 5 coppie.
Lanario Falco biarmicus M reg ?, B ? (1995), W irr?

In Europa, è specie nidificante in decremento (Staneva $\&$ Burfield, 2017). In periodo riproduttivo rinvenuto in aree aperte naturali o coltivate purché dotate di anfratti e su dirupi, dal livello del mare ad oltre $1100 \mathrm{~m}$ s.l.m., mentre in inverno, specialmente giovani e immaturi, tendono a disperdersi frequentando prati, pascoli e le zone umide più vicine alla linea di costa (Brichetti \& Fracasso, 2003). In drammatico calo come nidificante in Toscana nel periodo 2014-2016 (Pezzo et al., 2016). Nel PRM, per quanto piuttosto elusiva e perciò di difficile osservazione, è verosimilmente una specie di recente comparsa in inverno, con maggiore frequentazione dell'area protetta a partire dalla stagione 1999/00 (Giovacchini, 2006). Nel 1995 osservazioni regolari durante tutto l'anno tra Collelungo e la Palude della Trappola, dove era possibile la nidificazione in una zona prossima alla Grotta dello Scoglietto (G. Sammuri; R. Nardi; GA; PG). Nei successivi 1998 e 2003 rilevata la ripetuta presenza, tra febbraio-aprile, di singoli individui adulti (GA; PG). Ancora, tra le più recenti osservazioni: 1 giovane il 31/08/2011 a Castel Marino, Alberese (C. Martelli in GOM-List); 1 ind. il 21/12/2011 a Spergolaia, Alberese (PG); 1 ind. il 27 e 28/03/2012 alle Saline di San Paolo, foce del fiume Ombrone (C. Martelli in GOM-List); 1 ind. il 08/11/2015 alle Macchiozze, Alberese (PG).

\section{Sacro Falco cherrug M irr, W irr}

In Europa, è specie nidificante in incremento (Staneva $\&$ Burfield, 2017). Durante le migrazioni e lo svernamento frequenta zone aperte, specialmente ambienti xerici (Brichetti \& Fracasso, 2003). Segnalazioni prevalentemente invernali provenienti dal Centro Italia e dalla Sicilia (Cauli \& Genero, 2017). Nel PRM, precedentemente ritenuto accidentale con una sola segnalazione: 1 ind. il 06/11/2004 (R. Nardi in Mellone et al., 2005). Successivamente, raccolte segnalazioni invernali anche in zone immediatamente esterne all'area protetta: 1 ind. il 02/02/2005 tra Alberese e Rispescia (R. Nardi); 1 individuo nell'inverno 2005/06 o 2006/07 nel PRM (GA); forse 1 ind. il 19/01/2009 alle Macchiozze, Alberese (A. Corso in EBN-Italia). In migrazione: 1 ind. il 27/10/2009 in volo sopra i Monti dell'Uccellina, un animale slovacco munito di dispositivo satellitare e soprannominato "Lucia" (P. Harris in EBN-Italia); 1 ind. il 11/09/2011 alle Poste del Granduca, Alberese (C. Martelli in GOM-List).

\section{Falco pellegrino Falco peregrinus SB, M reg, W reg}

In Europa, è specie nidificante in incremento (Staneva $\&$ Burfield, 2017). Durante il periodo riproduttivo è rilevato più facilmente dal livello del mare a $1400 \mathrm{~m}$ s.l.m., distinguendosi nei settori costieri per essere rinvenuto preferibilmente nelle zone con falesie, mentre nelle aree montuose o collinari in corrispondenza di pendii rocciosi dominanti ed ancora in alcuni centri urbani, dove occupa specialmente torri e palazzi elevati; durante le migrazioni e lo svernamento frequenta anche pianure coltivate, pascoli e zone umide (Brichetti \& Fracasso, 2003). Nel PRM, segnalato nidificante con una coppia in un'area occupata da oltre tre decenni, in posizione dominante sulle falesie dei Monti dell'Uccellina (GA). Constatata la predazione 
del rapace su Accipiter nisus (Anselmi \& Corsi, 1992). Nel 1997 individuato tra il Salto del Cervo e lo Scoglietto di Alberese un secondo sito riproduttivo, poi abbandonato nel 1998 (GA). Negli anni 2002 e 2005 successo riproduttivo della coppia con 3 giovani involati, così come nel decennio successivo (GA). Incremento della popolazione in inverno, stagione già in parte interessata da comportamenti di coppia, come i voli nuziali: ad es. 1 coppia il 02/02/1998 a Cala di Forno (PG e D. Germani). Tra le più recenti osservazioni raccolte in questo periodo dell'anno: 1 ind. appartenente alla ssp. calidus il 10/12/2011 alla foce del fiume Ombrone (D. Brendan su Ornitho.it); 1 ind. il 16/01/2015 in volo rapido sopra i ruderi dell'abbazia di San Rabano, Alberese, a caccia di Columba livia [var. domestica] (C. Martelli, PG e P. Arrigucci); 1 ind. il 07/12/2015 alle Macchiozze (PG). Stimabile una presenza minima di 1 coppia nidificante.

\section{PSITTACIFORMES}

\section{Psittaculidae}

\section{Parrocchetto dal collare Psittacula krameri A-2}

Occasionale nell'area di studio. 1 ind. il 06/06/1994 al confine nord del PRM a Principina Mare (GA). Si aggiunge ora: 1 ind. il 10/05/2012 a Pian di Barca, Alberese (M. Simoni). Probabile provenienza da località (ad es. Follonica) in cui risulta naturalizzato (Andreotti et al., 2001; Baccetti et al., 2014).

\section{Pappagallino ondulato Melopsittacus undulatus A-1}

Occasionale nell' area di studio. Specie precedentemente non riportata (Giovacchini, 2006). 1 ind. il 21/07/2001 rinvenuto ferito sulla strada provinciale, Alberese (fonte: Centro Recupero Fauna Selvatica, Semproniano, Grosseto). Probabile provenienza domestica (Baccetti et al., 2014).

\section{PASSERIFORMES}

\section{Laniidae}

\section{Averla piccola Lanius collurio $\mathrm{M}$ reg, B}

In Europa, è specie nidificante stabile (Staneva \& Burfield, 2017). In Italia, è nidificante "Vulnerabile" (Peronace et al., 2012). Si riproduce preferibilmente tra 100 e $1300 \mathrm{~m}$ s.l.m., in ambienti aperti, incolti con cespugli, vigneti, frutteti, alberi sparsi, con abbondanza di insetti, dove per cacciare utilizza posatoi dominanti come palizzate o cavi sospesi (Brichetti \& Fracasso, 2011). Nel PRM, negli anni, presenze puntiformi, specialmente in luoghi caratterizzati da ambienti agricoli diversificati; contattato anche in vicinanza di pascoli con zone umide. Circa dal 2012, la specie mostra, come Lanius minor e $L$. senator, un evidente crollo della popolazione con contrazione dell'areale, in particolare nel settore costiero, dove nel secolo scorso veniva rilevata diffusamente e dove, nel 2005, era stimata una popolazione di 5-10 coppie (Farina \& Martelli, 1979; Giovacchini, 2006). Nella zona nord dell'area protetta risulta oggi scomparsa dalla Tenuta di
San Carlo mentre viene ancora rilevata nella Tenuta di Torre Trappola, in ambienti caratterizzati da pascoli magri con cespugliati e zone umide circostanti: 1 maschio il 27/05/2017 lungo la strada del mare (PG e A. Picciau). A sud della foce del fiume Ombrone: 1 giovane dell'anno il 10/08/2011 alle Saline di San Paolo (C. Martelli in GOMList). Altrove, le presenze non sono sempre imputabili a individui nidificanti. Stimabile una presenza minima di 1-5 coppie nidificanti. Distribuzione dei siti in periodo riproduttivo in Fig. 44.

\section{Averla cenerina Lanius minor $\mathrm{M}$ reg, B}

In Europa, è nidificante in decremento (Staneva \& Burfield, 2017). In Italia, è nidificante "Vulnerabile" (Peronace et al., 2012). Predilige ambienti aperti di pianura e collina caratterizzati da alberi sparsi o a gruppi in zone soleggiate ed in prossimità di colture estensive, pascoli o allevamenti zootecnici, generalmente sino 200-300 m s.l.m. e dove vi sia abbondanza di insetti (Brichetti \& Fracasso, 2011). Nel PRM, successivamente alla stagione riproduttiva 2007, presenze limitate al territorio compreso tra il confine nord di Principina Mare e Magazzini di Alberese, con sensibile riduzione dell'areale. Tra le più recenti osservazioni si segnala: 1 ind. il 30/05/2008 lungo la strada provinciale della Trappola (G. Ceccolini e A. Cenerini); 1 ind. il 14/06/2008 in località Magazzini, Alberese (PG); 1 ind. il 12/06/2012 nei pressi della foce del fiume Ombrone (PG); 1 ind. il 27/05/2017 in allarme su posatoio all'interno della Tenuta di Torre Trappola (PG e A. Picciau). Stimabile una presenza minima di 1-3 coppie nidificanti. Distribuzione dei siti in periodo riproduttivo in Fig. 45.

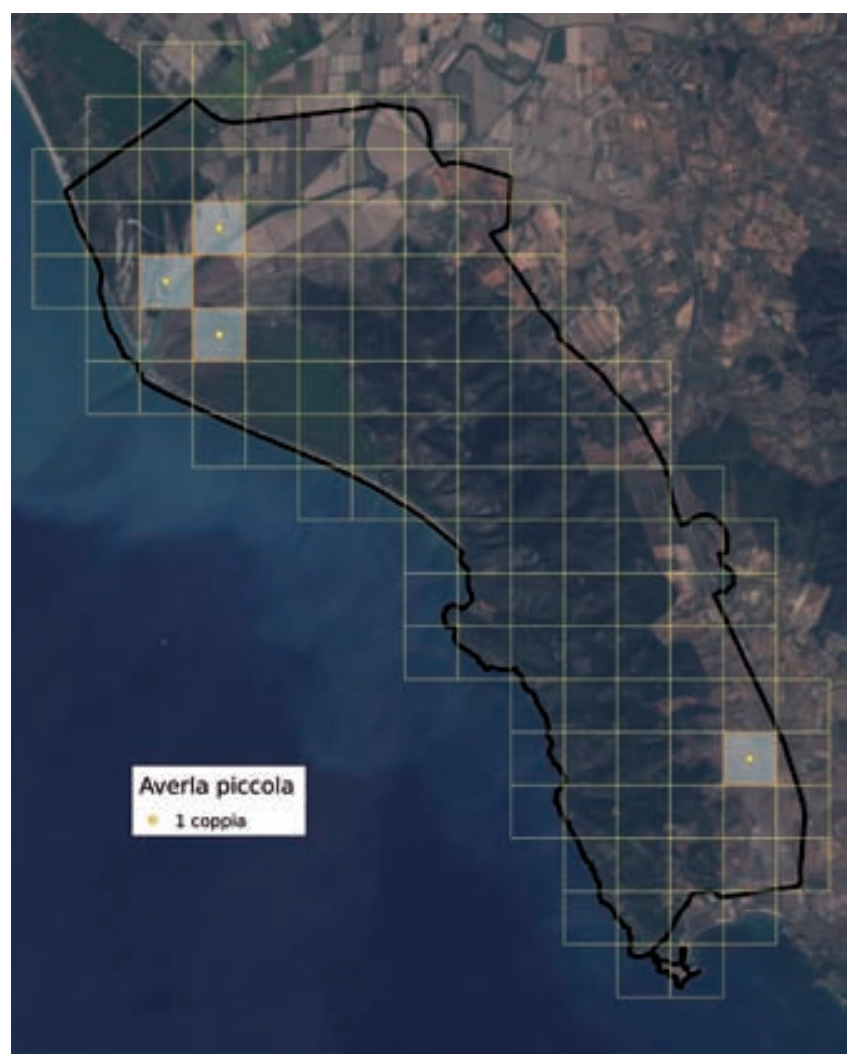

Fig. 44 - Siti di presenza di averla piccola in periodo riproduttivo. / Sites of presence of Red-backed Shrike in breeding season (2008-2017). 


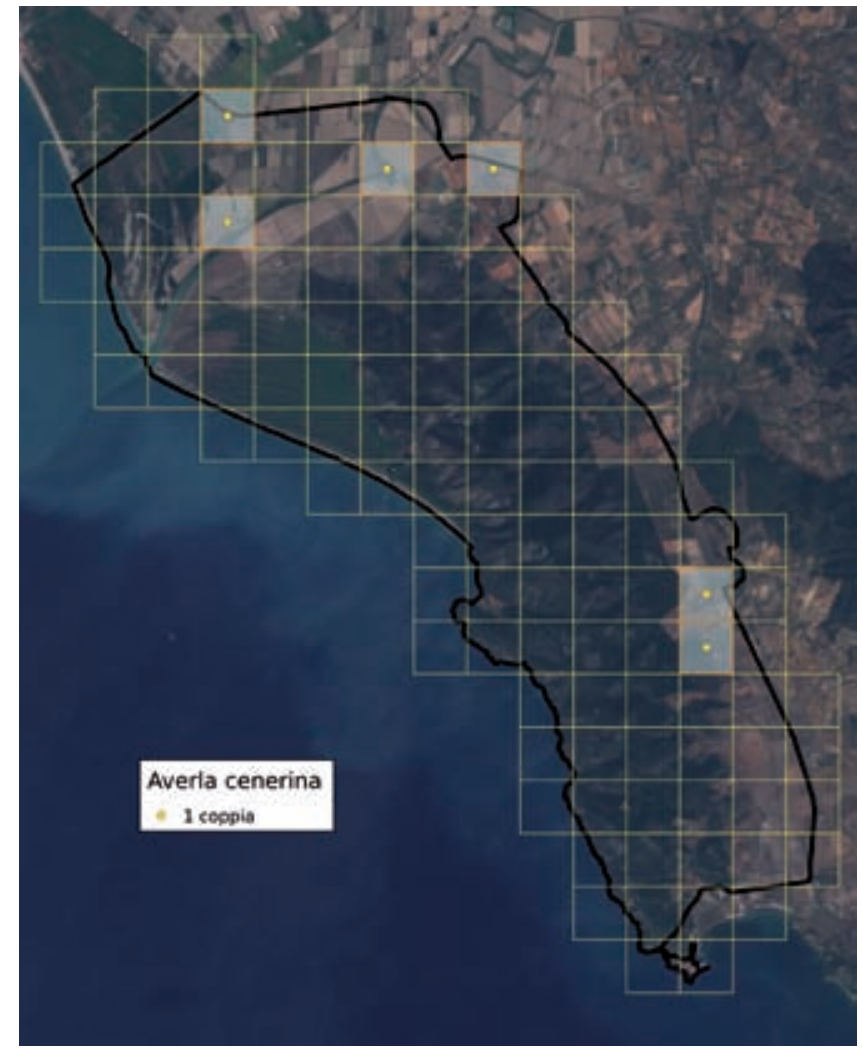

Fig. 45 - Siti di presenza di averla cenerina in periodo riproduttivo. / Sites of presence of Lesser Grey Shrike in breeding season (20012017).

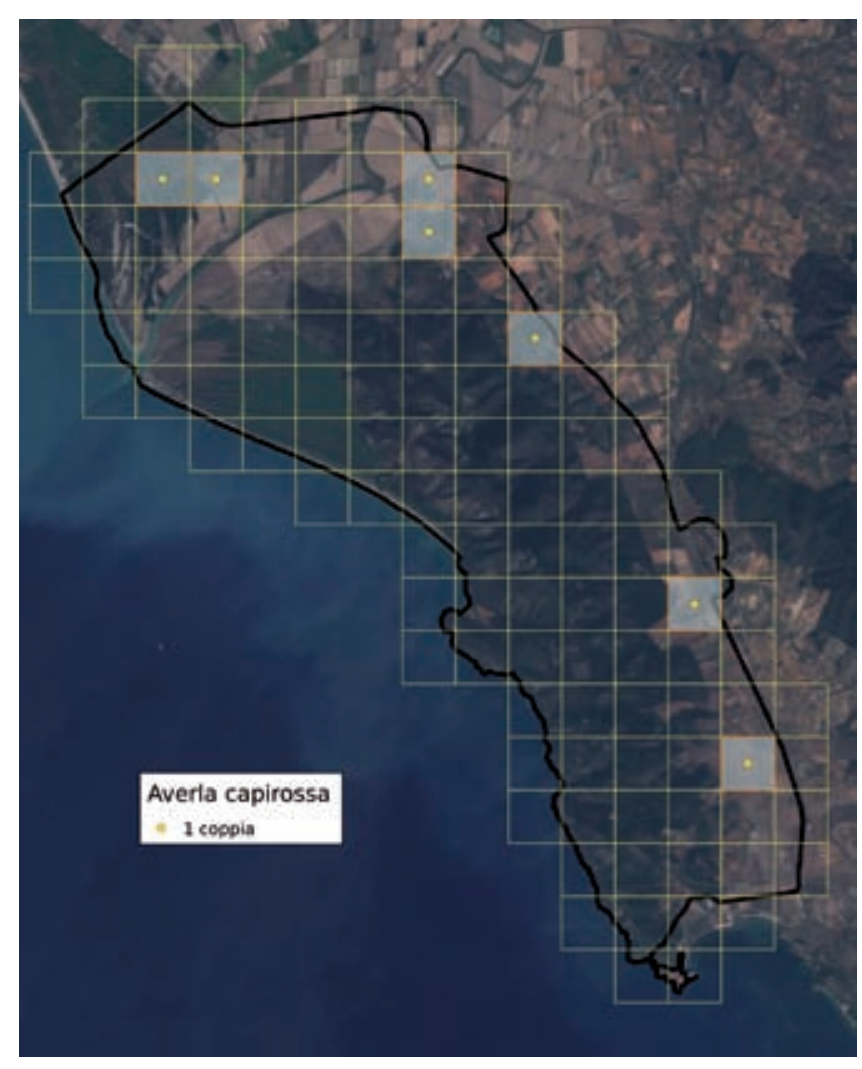

Fig. 46 - Siti di presenza di averla capirossa in periodo riproduttivo. / Sites of presence of Woodchat Shrike in breeding season (2001-2017).
Averla maggiore Lanius excubitor A-1

Accidentale. 1 ind. nel periodo compreso tra il 5 e 24/12/1986 a Marina di Alberese (S. Benucci, D. Biagini e GA in Arcamone \& Tellini, 1987).

Averla capirossa Lanius senator $\mathrm{M}$ reg, B

In Europa, è nidificante in decremento (Staneva \& Burfield, 2017). In Italia, è nidificante "In Pericolo" (Peronace et al., 2012). Predilige ambienti aperti in zone soleggiate di pianura e collina con abbondanza di insetti, cespugliati con alberi sparsi, in prossimità di colture estensive, pascoli e allevamenti zootecnici, generalmente sino a 600-800 m s.l.m. (Brichetti \& Fracasso, 2011). Nel PRM, rilevata una evidente contrazione del proprio areale rispetto al primo decennio di questo secolo, con scomparsa da località tradizionalmente frequentate, per esempio: Collecchio, Tenuta di San Carlo e Podere Cava Zuccherina (PG; GA). Segnalazioni più recenti: 1 ind. il 03/06/2012 nei pressi del Ponte delle Carriole, Tenuta di Torre Trappola (F. Farsi e PG); 1 ind. nel giugno 2017 in località La Bernarda, Alberese (F. Pezzo); 1 ind. il 27/04/2018 nei pressi di Spolverino (GA). Stimabile una presenza minima di 1-5 coppie nidificanti. Distribuzione dei siti in periodo riproduttivo in Fig. 46.

\section{Oriolidae}

Rigogolo Oriolus oriolus $\mathrm{M}$ reg, B

In Europa, è specie nidificante con trend incerto (Staneva \& Burfield, 2017). Si riproduce in zone boscose di latifoglie formanti fitte canopee nei pressi di corpi d'acqua, ma anche in frutteti, coltivi alberati con siepi ed in aree urbane e suburbane, generalmente sino a 500-600 m s.l.m. (Brichetti \& Fracasso, 2011). Nel PRM, appare ben rappresentato lungo il tratto ripariale nord del fiume Ombrone, sino a Torre Trappola. Nella Pineta Granducale dell'Uccellina è relativamente diffuso, per quanto con valori di dominanza e abbondanza bassi (Pezzo, 2012). Nel periodo della nidificazione segnalato anche nei pressi del Condotto di Alberese, Torre Bassa e della Valentina (PG; GA; U. Boldorini; G. F. Martini; L. Buratta). Stimabile una popolazione minima di 15 coppie nidificanti.

\section{Corvidae}

\section{Ghiandaia Garrulus glandarius SB}

In Europa, è nidificante in incremento (Staneva \& Burfield, 2017). Si riproduce in ambienti boschivi di varia natura e composizione, con predilezione per quelli di latifoglie, soprattutto querceti, ma localmente anche in pinete e boschi planiziali, risultando più diffusa dal livello del mare a 1400-1500 m s.l.m. (Brichetti \& Fracasso, 2011). Nel PRM, presenze piuttosto uniformi in ambienti adatti, con densità stagionali variabili in relazione ai vari habitat utilizzati: ad esempio, in inverno la densità è massima (15-150 ind $/ \mathrm{km}^{2}$ ) nella macchia caratterizzata da lecci a portamento arboreo (Rolando et al., 1997; Rolando, 1998). I movimenti, le dimensioni e la disposizione degli home range sembrano dipendere dai fattori trofici (Patterson et al., 1991; Rolando et al., 1995). Nelle stagioni di nidificazione 2000-2004 rilevati 48 territori su un 
totale di 91 punti di ascolto, con una frequenza relativa pari a 5,37\%. Stimabile una popolazione minima di 200 coppie nidificanti.

\section{Gazza Pica pica SB}

In Europa, è nidificante stabile (Staneva \& Burfield, 2017). Si riproduce in ambienti alberati di varia composizione e struttura, in parchi, giardini e orti urbani, più diffusa sino a 600-700 m s.l.m. (Brichetti \& Fracasso, 2011). Nel PRM, sembra preferire gli ambienti con soprassuoli radi, specialmente i margini delle pinete (Pezzo, 2012). Nelle stagioni di nidificazione 2000-2004 rilevati 34 territori su un totale di 91 punti di ascolto, con una frequenza relativa pari a $4,0 \%$. Stimabile una popolazione minima di 80 coppie nidificanti.

\section{Taccola Coloeus monedula SB, M reg, W reg}

In Europa, è nidificante stabile (Staneva \& Burfield, 2017). In periodo riproduttivo è specie rilevata prevalentemente in situazioni sinantropiche, ma non disdegna gli ambienti naturali con spazi aperti ricchi di cavità naturali, ruderi, torri, generalmente sino a 700-800 m s.l.m.; movimenti dispersivi e formazione di gruppi anche di una certa consistenza nel resto dell'anno (Brichetti \& Fracasso, 2011). Nel PRM, presenza regolare con colonie "storiche" nei pressi della grotta dello Scoglietto ed a Torre Bassa, dove convive con Columba livia [var. domestica], entrambi siti caratterizzati da scarso disturbo antropico. Almeno nell'anno 2000 nidificazione di una coppia in una cavità di Pinus pinea nella Tenuta di San Carlo (PG). Negli anni la specie si è vista occupare con successo i nidi artificiali predisposti per Coriacias garrulus (Corsi \& Anselmi, 1994; Pezzo, 2012). In inverno, negli anni 20002009 si registra un andamento fluttuante dei contingenti, con riferimento specialmente all'area di Torre Bassa ove si rilevano cifre comunque comprese tra 20-70 individui a gennaio (PG). Specialmente in questo periodo dell'anno quando agli individui sedentari si aggiungono gli svernanti, la specie è osservata anche in gruppi nei pressi dei pascoli con bestiame a Spergolaia, Macchiozze e Rimessini (PG). Stimabile una popolazione minima di 20 coppie nidificanti.

\section{Corvo comune Corvus frugilegus A-1}

In Europa, è nidificante in decremento (Staneva \& Burfield, 2017). Durante lo svernamento e con le migrazioni frequenta preferibilmente zone di pianura e fondovalle ricche di seminativi e prati da sfalcio, con presenza di filari alberati, vigneti e frutteti dove forma concentrazioni di decine o centinaia di individui (Brichetti \& Fracasso, 2011). La Toscana non è compresa nell'attuale areale di svernamento (Bogliani, 1985; Arcamone et al., 2011). Nel PRM, osservazioni di 1-2 individui riferite unicamente all'inverno 1993/94: 1 adulto il 16 e 20/12/1993 nei seminativi delle Macchiozze (PG); 2 adulti posati a terra il 14/02/1994 nella Tenuta di Torre Trappola (Giovacchini, 19951). Ancora nelle stagioni invernali comprese tra il 1935 e il 1940, in Alberese, era oggetto di interventi finalizzati al contenimento dei danni procurati alle colture (A. Innocenti, com. pers.). 1 maschio catturato nel gennaio 1884 a Talamone, donato nel 1888 al National Museum of Natural History di Washington (catalogo delle cessioni reperti del Museo Zoologico La Specola, Firenze, F. Barbagli, com. pers.).

Cornacchia grigia Corvus cornix SB, M reg, W reg

Nidifica in ambienti alberati diversificati, naturali o artificiali, dalle pinete litoranee ai pioppeti industriali sino alle praterie di media montagna adattandosi agli ambienti fortemente antropizzati, dal livello del mare ai 1700-1900 m s.l.m. (Brichetti \& Fracasso, 2011). Nel PRM, la specie viene segnalata diffusamente nella Pineta Granducale dell'Uccellina nidificando nelle parti più alte delle chiome (Farina \& Martelli, 1979; Pezzo, 2012). Una frazione della popolazione dell'area protetta è verosimilmente costituita da individui sedentari. La riproduzione viene ugualmente segnalata nei settori orientali, in vicinanza di contesti caratterizzati da ambienti rurali coltivati o non, più spesso con presenze di siepi e modesta copertura arborea. Nel resto dell'anno, presenze regolari di gruppi di una certa consistenza numerica, anche oltre 20 individui. Nell'area protetta stimabile una popolazione minima di 35 coppie nidificanti.

\section{Corvo imperiale Corvus corax $\mathrm{M}$ irr, B ext}

In Europa, è nidificante in incremento (Staneva \& Burfield, 2017). Si riproduce su falesie e pareti rocciose strapiombanti, quindi lungo le coste marine e nelle aree interne, comunque in vicinanza di zone aperte in cui cacciare, con l'occupazione territoriale del sito durante tutto l'anno da parte della coppia, dal livello del mare a circa 2000 m s.l.m. (Brichetti \& Fracasso, 2011). Abbastanza di recente, si ipotizzava la riproduzione nel PRM sui Monti dell'Uccellina (Di Carlo, 1981). Residente nidificante con una coppia dalla stagione 1993, con la prima indicazione di occupazione stabile dell'area protetta raccolta nel precedente anno: 2 ind. il 15/01/1992 in volo sopra Poggio La Murcia, Talamone (PG e I. Nerozzi). Sino alla stagione riproduttiva 2001 utilizzata per il nido una falesia a Punta del Corvo, Talamone (PG e GA). Nel 2002 spostamento del sito in località Porticciolo, Talamone, fino alla scomparsa come nidificante dai Monti dell'Uccellina avvenuta con la conclusione della stagione 2006 (GA). Nel corso degli anni radunate alcune segnalazioni riferite a voli condotti dagli adulti con i giovani, ad es. 4 ind., di cui 2 giovani, in volo il 15/06/1997 a Punta del Corvo (PG); 4 ind., di cui 2 giovani, in volo il 22/06/2000 a Vaccareccia (GA). Nel periodo 1992-2006 la specie è stata comunque rilevata in tutta l'area protetta durante voli di spostamento verso le zone di alimentazione poste nel PRM, come il Lasco di Alberese, Macchiozze, Trappola, foce del fiume Ombrone; occasionalmente osservazioni della specie anche in movimenti verso l'esterno del PRM, ad es. 1 ind. il 08/10/1998 in volo sopra la Trappola in direzione nord-est (PG); 2 ind. il 14/11/2003 in volo sopra l'abbazia di San Rabano in direzione est (R. Ferrari).

\section{Paridae}

Cincia mora Periparus ater $\mathrm{M}$ irr, $\mathrm{W}$ irr

In Europa, è nidificante in incremento (Staneva \& Burfield, 2017). Migratrice numericamente molto flut- 
tuante con spostamenti saltuari di tipo invasivo; lo svernamento nelle aree non interessate dalla nidificazione riguarda verosimilmente anche individui locali in erratismo verticale, osservati prevalentemente in parchi urbani, boschi collinari e di pianura a conifere pure o miste (Brichetti \& Fracasso, 2011). Prime "apparenti" segnalazioni per il PRM: 1-2 ind. il 04/01/1997 in località Campo al Pino, Alberese, ed ancora 1 ind. il 31/01/1997 a Cala di Forno (Giovacchini, 1999). Successivamente rilevata anche negli inverni 2005/06 e 2006/07 (L. Bordignon e PG; A. Vezzani in Arcamone \& Puglisi, 2008). Le osservazioni fanno prevalentemente riferimento a 1-3 individui rinvenuti nella pineta a Principina Mare, nei pressi della foce del fiume Ombrone e ai Rimessini: 3 ind. il 25/11/2005 in questa ultima località (PG). Successive indicazioni riferite all'inverno $2017 / 18$ con un massimo di 4 ind. il 04/01/2018 alla foce del fiume Ombrone (M. Fedi su Ornitho.it). Massima concentrazione assoluta per il PRM: 5 ind. il 09/12/2005 nella pineta al confine con Principina Mare (PG). Una segnalazione storica, antecedente all'istituzione dell'area protetta: 1 ind. il 27/12/1972 nella pineta di Alberese (C. Castellani, ined.).
Cincia dal ciuffo Lophophanes cristatus A-1

Accidentale. Precedentemente non rinvenuta (Giovacchini, 2006). Almeno 2 ind. il 03/11/2016 nella pineta al confine con Principina Mare (M. Grylle su Ornitho.it; Fig. 47).

Cinciarella Cyanistes caeruleus SB, M reg ?

In Europa, è nidificante in incremento (Staneva \& Burfield, 2017). Si riproduce in zone boscose estese decidue o sempreverdi, pure o miste, con alberi maturi e radure, più diffusa sino a 1200-1300 m s.l.m.; in inverno movimenti di erratismo verticale con apporto di migratori da aree esterne all'Italia, frequenta anche ambienti con scarsa copertura arborea come frutteti o siepi (Brichetti \& Fracasso, 2011). Nel PRM, è specie con basse densità, piuttosto localizzata durante tutto l'anno, presente preferibilmente nei complessi forestali con latifoglie più freschi, boschetti misti e nei pascoli con filari di caducifoglie, come quelli dell'abbazia di San Rabano, Condotto, Lasco di Alberese, Pancotto e Valentina di Talamone (GA; S. Celletti; PG). Nei periodi riproduttivi compresi tra il 2000-2004 concentrazione massima di 4 individui nell'area dell'abbazia di San Rabano (PG). Una frazione della popolazione

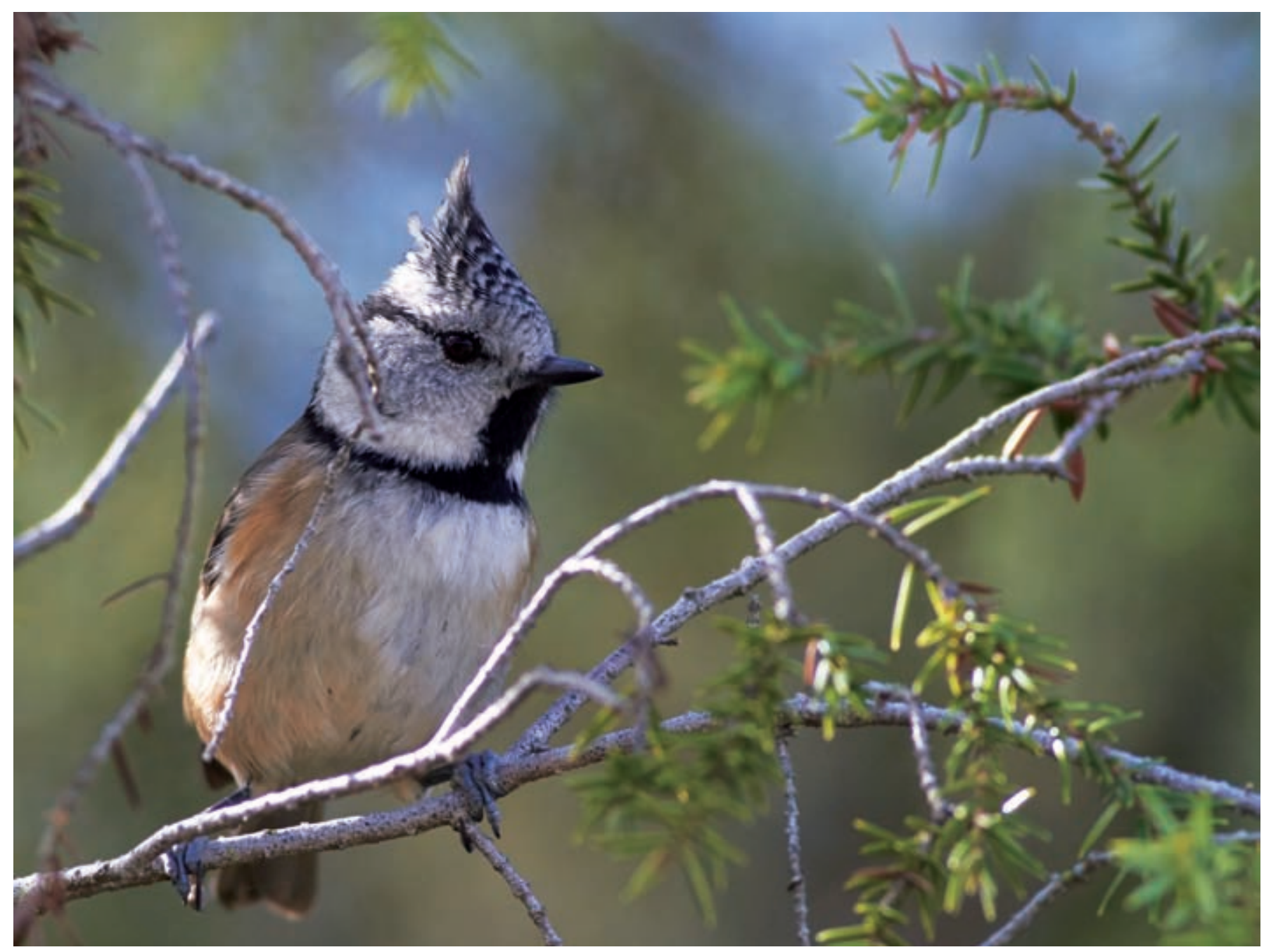

Fig. 47 - Cincia dal ciuffo il 03/11/2016 nella pineta di Principina Mare. / Crested Tit on the $3^{\text {rd }}$ November 2016 in the Principina Mare pinewood (Foto / Photo Magnus Grylle). 
è verosimilmente costituita da individui sedentari. Nelle stagioni di nidificazione 2000-2004 censiti 13 territori su un totale di 91 punti di ascolto, con una frequenza relativa pari a $1,55 \%$. Nell'area protetta stimabile una popolazione minima di 30 coppie nidificanti.

\section{Cinciallegra Parus major SB, M reg?}

In Europa, è nidificante in incremento (Staneva \& Burfield, 2017). La specie si riproduce in ambienti di varia natura, generalmente con alberi maturi provvisti di cavità idonee, più facilmente in boschi di latifoglie mesofile, pinete, frutteti, boschi ripariali e parchi urbani, risultando più diffusa sino a 1000-1200 m s.l.m.; in inverno frequenta sostanzialmente gli stessi ambienti (Brichetti \& Fracasso, 2011). Nel PRM, durante l'anno, è specie decisamente più diffusa di Cyanistes caeruleus. Una frazione maggioritaria della popolazione è verosimilmente costituita da individui sedentari. Risulta dominante nella comunità degli uccelli nidificanti nella Pineta Granducale dell'Uccellina, occupando con successo anche i nidi artificiali predisposti per Coriacias garrulus (Pezzo, 2012). Nelle stagioni di nidificazione 2000-2004 censiti 39 territori su un totale di 91 punti di ascolto con una frequenza relativa pari a $4,66 \%$. Nell' area protetta stimabile una popolazione minima di 250 coppie nidificanti.

\section{Remizidae}

Pendolino Remiz pendulinus $\mathrm{M}$ reg, $\mathrm{W}$ reg, B ext

In Europa, è nidificante con trend incerto (Staneva $\&$ Burfield, 2017). Si riproduce in zone umide di acqua dolce, salmastra, fiumi, con vegetazione igrofila ripariale, così come in aree periurbane con piante arboree collocate nei pressi di canali o fossi, più facilmente sino a 200-300 $\mathrm{m}$ s.l.m.; in inverno e in migrazione frequenta una vasta gamma di ambienti, all'interno di zone umide, canali, fossi, purchè provvisti di canneti, o poco distanti da corpi idrici, più facilmente con alberature (Brichetti \& Fracasso, 2011). In Toscana, nel periodo della riproduzione, è specie localmente in calo (Brichetti \& Grattini, 2010). Nel PRM, è scomparso come nidificante dalla stagione 2003. Sino all'anno precedente rilevate infatti 1-2 coppie lungo il fiume Ombrone (R. Ragazzini; M. C. Paolini; M. Simoni). In questo periodo dell'anno massime concentrazioni negli anni 1985-1990 con 4 coppie lungo 1,2 km del fosso che scorre nei pressi della strada per Marina di Alberese (GA e PG). Individuo inanellato: 1 ind. catturato il 17/10/1972 alla foce del fiume Ombrone, Grosseto, era stato inanellato il 06/07/1972 a Ladek, Polonia (Bendini \& Spina, 1990). Segnalazioni pregresse all'istituzione dell'area protetta, con nidi rinvenuti nella Tenuta di San Carlo (Di Carlo, 1981) (Fig. 48).

\section{Alaudidae}

Tottavilla Lullula arborea SB, M reg, W reg

In Europa, è nidificante in incremento (Staneva \& Burfield, 2017). Nidifica preferibilmente in ambienti erbosi in leggera pendenza posti ai margini di boschetti e cespugli sparsi occupando oliveti, vigneti e pascoli alberati, principalmente tra i 200-1000 m s.l.m.; in inverno e durante le migrazioni presente anche in zone litoranee occupando una vasta gamma di tipologie ambientali (Brichetti \& Fracasso, 2007). Nel PRM, è specie comunque a distribuzione localizzata; questa, nel periodo riproduttivo, è riconducibile ai settori centro meridionali dell'area protetta, quasi sempre a quote prossime al livello del mare. Una frazione della popolazione è verosimilmente costituita da individui sedentari. La specie, durante la stagione di nidificazione viene per esempio segnalata ai piedi del sistema montuoso dei Monti dell'Uccellina nei pressi delle località Vergheria e Bernarda in Alberese, così come nell'area di Fonte Lepre della Valentina ed ancora ai margini dei boschi cedui in località Pancotto, questi ultimi nella zona di Talamone (GA; PG). In inverno, rilevati anche gruppetti composti per un massimo di 4-5 individui nella zona della Valentina a Talamone (PG). Nelle stagioni di nidificazione 2000-2004 censiti 10 territori su un totale di 91 punti di ascolto con una frequenza relativa pari a $1,20 \%$. Nell'area protetta stimabile una popolazione minima di 10 coppie nidificanti.

\section{Allodola Alauda arvensis SB, M reg, W reg}

In Europa, è nidificante in decremento (Staneva \& Burfield, 2017). In Italia, è nidificante "Vulnerabile" (Peronace et al., 2012). Si riproduce in ambienti aperti, dalla pianura ai $2000 \mathrm{~m}$ s.l.m. circa, dove occupa per esempio, coltivi intensivi, alte praterie montane, prati e pascoli, margini di zone umide e zone suburbane; in inverno rilevata anche nelle stoppie di cereali e ai margini delle sedi stradali (Brichetti \& Fracasso, 2007). Nel PRM, è specie a distribuzione piuttosto ampia, occupando tra l'altro habitat tra loro molto diversi come le praterie alofile di Bocca d'Ombrone e i coltivi intensivi posti quasi sempre nel settore orientale dell'area protetta. Una frazione della popolazione è verosimilmente costituita da individui sedentari a cui si aggiungono in inverno contingenti svernanti. Oltre a Bocca d'Ombrone, durante la stagione di nidificazione la specie viene per esempio segnalata nei prati

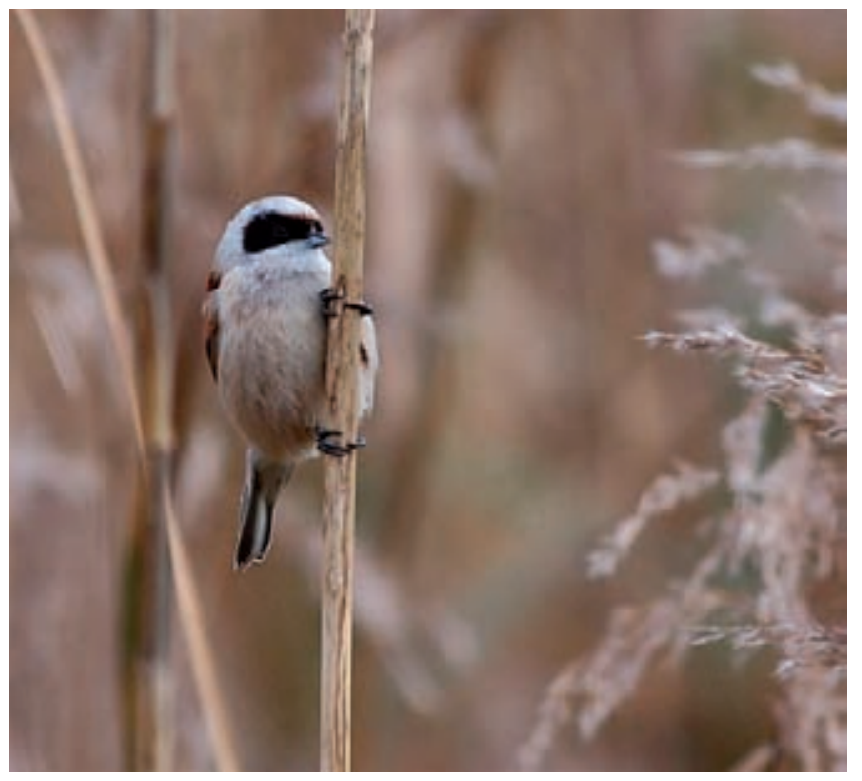

Fig. 48 - Pendolino nella Palude della Trappola. / Penduline Tit in the Trappola's marsh (Foto / Photo Stefano Laurenti). 
pascoli delle Macchiozze con densità pari a 4,3 coppie/10 ha (PG), nei seminativi delle Pianacce ed ancora nell'area della ex cava di parete della Burraia in Alberese e, nel settore nord del PRM, diffusamente nella Tenuta di Torre Trappola, mentre a sud compare per esempio negli incolti del Collecchio, nei coltivi della Valentina così come nei seminativi della bonifica di Talamone (GA; PG). Nelle stagioni di nidificazione 2000-2004 censiti 40 territori su un totale di 91 punti di ascolto con una frequenza relativa pari a $4,77 \%$. Nell'area protetta stimabile una popolazione minima di 40 coppie nidificanti.

\section{Cappellaccia Galerida cristata SB, M irr}

In Europa, è nidificante in decremento (Staneva \& Burfield, 2017). La riproduzione interessa zone aperte, incolte, aride, sabbiose o pietrose, pascoli, anche ai margini di aree urbane, così come frutteti, coltivazioni di ortaggi e cereali, alvei fluviali, margini di cave dismesse, risultando più diffusa sino a 500-600 m s.l.m.; in inverno frequenta anche litorali sabbiosi, argini fluviali, margini di boschetti, campi arati e sedi stradali (Brichetti \& Fracasso, 2007). Nel PRM, è specie a distribuzione piuttosto ampia, occupando spesso nicchie simili a quelle segnalate per Alauda arvensis per quanto in contesti caratterizzati anche da spazi aperti di minore estensione. La popolazione è verosimilmente costituita da individui sedentari a cui in inverno si aggiungono contingenti più facilmente in dispersione da aree locali che portano ad incrementarne la diffusione, rilevando gruppetti formati anche da 4-6 individui. È possibile che la forma rappresentata localmente sia attribuibile alla sottospecie di G. c. neumanni la cui distribuzione copre dalla Toscana all'area di Roma. Nel periodo della riproduzione viene segnalata, ad esempio, nei pascoli della foce del fiume Ombrone, nei frutteti delle Pianacce ed ancora negli incolti delle Macchiozze, così come nei pascoli della Tenuta delle Femminelle, Tenuta di San Carlo e Tenuta di Torre Trappola nonchè nei pressi della intera rete viaria poderale, da nord a sud (PG; GA; G. F. Martini; A. Gianni; U. Boldorini; I. Nerozzi). Nelle stagioni di nidificazione 2000-2004 censiti 47 territori su un totale di 91 punti di ascolto con una frequenza relativa pari a 5,61\%. Nell'area protetta stimabile una popolazione minima di 70 coppie nidificanti.

\section{Calandrella Calandrella brachydactyla $\mathrm{M}$ reg, B}

In Europa, è nidificante con trend sconosciuto (Staneva \& Burfield, 2017). In Italia, è nidificante "In Pericolo" (Peronace et al., 2012). Si riproduce in zone aperte, calde, costiere ed interne, dove occupa dune sabbiose, greti fluviali, margini di zone umide, pascoli ma anche coltivi erbacei nei primi stadi vegetativi purché su suoli ben drenati (Brichetti \& Fracasso, 2007). Nel PRM, presenze localizzate di singoli individui in canto, con l'occupazione di habitat che nel periodo della riproduzione spaziano dai pascoli magri della foce del fiume Ombrone alle coltivazioni orticole o a cereali nei pressi di Spergolaia o in quelle immediatamente esterne all'area protetta. Individui in canto, per esempio, anche nei pressi del Podere Merano in Alberese, in località Tombolello e nel Podere Ernesta a Talamone (PG). Tra le più recenti segnalazioni: 1 ind. il 27/05/2015 nei pascoli della Tenuta di Torre Trappola (PG e A. Picciau); 1 ind. il 06/06/2017 alla foce del fiume Ombrone (PG). Nel PRM, stimabile una popolazione minima di 2-4 coppie nidificanti. Prima dell'istituzione dell'area protetta forse anche più comune: 3 ind. il 28/06/1967 e 12 ind. il 30/05/1975 alla Trappola (Di Carlo, 1981).

\section{Calandra Melanocorypha calandra B ext}

In Europa, è nidificante in decremento (Staneva \& Burfield, 2017). Si riproduce in ambienti caldi, secchi, pietro$\mathrm{si}$, in zone pianeggianti e soleggiate, dove occupa incolti con vegetazione diradata, localmente coltivi estensivi e margini disseccati di zone umide (Brichetti \& Fracasso, 2007). In Toscana mancano da alcuni decenni prove certe di riproduzione (Baccetti \& Meschini, 1986). L'area del PRM ha verosimilmente ospitato una popolazione riproduttrice sino all'anno della sua istituzione, scomparendo poco dopo: circa 15-20 ind. nel maggio e giugno 1975 alla Trappola (Di Carlo, 1981).

\section{Hirundinidae}

\section{Topino Riparia riparia $\mathrm{M}$ reg}

In Europa, è nidificante con trend incerto (Staneva \& Burfield, 2017). Durante le migrazioni rilevato per esempio ai margini di zone umide artificiali o naturali, corsi d'acqua, praterie e pascoli (Brichetti \& Fracasso, 2007). Nel PRM, le occasionali presenze registrate nel passato in periodo riproduttivo non risultano confortate da successive conferme (Baccetti \& Meschini, 1986). Se non osservato attentamente, possibilità di confusione con $D e-$ lichon urbicum o Ptyonoprogne rupestris. Nell'area protetta rinvenuto specialmente in occasione della migrazione primaverile, tra marzo-maggio, più spesso aggregato a Hirundo rustica o Delichon urbicum, con contingenti generalmente limitati al massimo a 9-13 individui. Per i due momenti migratori si citano ad es. 6 ind. in volo il 21/05/2002 alle Cetine, Trappola, insieme ad almeno 10 ind. di Delichon urbicum (GA e P. Arrigucci); 8 ind. il 21/09/2007 insieme ad oltre 60 ind. di Delichon urbicum e circa 5 ind. di Hirundo rustica, poi posatisi all'interno di un canneto scelto quale dormitorio nei pressi delle Macchiozze (PG).

\section{Rondine Hirundo rustica $\mathrm{M}$ reg, B, W irr}

In Europa, è nidificante in decremento (Staneva \& Burfield, 2017). In Italia, è nidificante "Quasi Minacciato" (Peronace et al., 2012). Si riproduce generalmente in fabbricati rurali in aree prossime a sistemi tradizionali di conduzione agricola e di allevamento del bestiame, ricchi di entomofauna, più diffusa sino a $1200-1300$ m s.l.m.; in inverno è rinvenuta più facilmente in zone pianeggianti costiere e ai margini di zone umide (Brichetti \& Fracasso, 2007). Nel PRM, registrata una progressiva tendenza alla diminuzione nel numero delle coppie nidificanti, in linea con l'andamento nazionale, per quanto ancora a metà degli anni '80 del secolo scorso risultava essere una delle specie più legate agli allevamenti zootecnici da cui non era mai assente (Rete Rurale Nazionale \& LIPU, 2015; I. Nerozzi, com. pers.). Segnalazioni riferite al periodo 2006-2018, in varie località per lo più con 1-10 coppie da Principina Mare a Talamone, in manufatti tradizionali 
quali magazzini, stalle ospitanti bestiame, maneggi, strutture semi abbandonate ad es. Tenuta di San Mamiliano, Tenuta di Torre Trappola, Tenuta di San Carlo, Spergolaia, Podere Cava Zuccherina, Podere Le Tofane, Tenuta del Collecchio e Podere San Giuseppe (GA; PG; G. F. Martini; M. Lunardi). Singole coppie nidificanti anche nelle strutture sottostanti ai ponti del Collettore Morelle, Chiavica alla foce del fiume Ombrone, Canale del Collecchio (GA; PG). Segnalazioni di individui attardatisi durante le migrazioni, ad es. 1 ind. il 24/11/1996 a Marina di Alberese (PG). Svernamento irregolare con segnalazioni riferite esclusivamente alla costa: 2 ind. il 23 o 24/12/1986 alla Trappola (Giovacchini, 1995c); 1 ind. il 12/12/1999 alla foce del fiume Ombrone (SL e PG); 1 ind. il 05/02/2003 a Principina Mare (M. Giunti in Arcamone \& Puglisi, 2006). Anomalie della colorazione del piumaggio: 1 ind. totalmente albino osservato da agosto a settembre 1996 a Spolverino, Alberese (GA e A. Gianni). Stimabile una popolazione minima di 20 coppie nidificanti.

\section{Rondine montana Ptyonoprogne rupestris $\mathrm{M}$ irr}

In Europa, è nidificante stabile (Staneva \& Burfield, 2017). Durante le migrazioni segnalata nei centri abitati, in prossimità di corsi d'acqua e di aree rocciose e nei pressi dei coltivi; la specie può formare in migrazione ed in inverno aggregazioni di alcune centinaia di individui (Brichetti \& Fracasso, 2007). Se non osservata attentamente, possibilità di confusione con Riparia riparia o Delichon urbicum. Nel PRM, registrate segnalazioni irregolari, perlomeno dal 1986. Si tratta di osservazioni che interessano l'intero comprensorio dell'area protetta: 1 ind. il 16/10/1995 alla Trappola (GA); 1 ind. il 13/03/2000 a Capo d'Uomo, Talamone (GA e PG); 3 ind. il 06/03/2015 in località Le Grotte, Alberese (GA e G. F. Martini). Segnalazioni non meglio precisate di nidificazione sui Monti dell’Uccellina (Di Carlo, 1981).

\section{Balestruccio Delichon urbicum $\mathrm{M}$ reg, B}

In Europa, è specie nidificante in decremento (Staneva $\&$ Burfield, 2017). In Italia, è nidificante "Quasi Minacciato" (Peronace et al., 2012). Si riproduce in ambienti antropizzati rurali e urbani, ricchi di siti idonei, risultando più diffuso sino a 1200-1300 m s.l.m. (Brichetti \& Fracasso, 2007). Nel PRM, la riproduzione è circoscritta a poche aree antropizzate su fabbricati rurali o ville, generalmente con un massimo di 3-4 coppie nel caso di siti isolati. Per esempio, nei decenni precedenti: nel 1986, su una villa a Capo d'Uomo, Talamone (PG); attualmente nella villa della Tenuta Granducale, Alberese, ed ancora nella struttura semi abbandonata della Tenuta del Collecchio (PG). Rilevato anche nei complessi rurali di Alberese, al confine interno dell'area protetta (vicinanze di Vergheria). Cospicui insediamenti sono registrati nelle frazioni immediatamente esterne di Alberese e Talamone. Segnalazioni di individui attardatisi durante le migrazioni, ad es. 1 ind. il 20/11/1992 alla foce del fiume Ombrone (PG). Tra le segnalazioni più precoci si ricorda: 8 ind. il 15/02/2004 in località Caprarecce, Talamone (GA); 1 ind. il 03/02/2013 in volo sul mare a Principina Mare (R. Gildi in EBN-Italia). Stimabile una popolazione minima di 10 coppie nidificanti.
Rondine rossiccia Cecropis daurica $\mathrm{M}$ irr ?, B irr

In Europa, è specie nidificante con tendenza alla stabilità (Staneva \& Burfield, 2017). In Italia, è nidificante "Vulnerabile" (Peronace et al., 2012). Si riproduce in ambienti caldi e secchi, aperti o accidentati, litoranei o dell'interno, generalmente con coppie sparse o isolate, con il sito preferibilmente sotto ponti di varia altezza, localmente in pareti rocciose, a volte in edifici abbandonati o porticati, comunque più diffusa sino a $300 \mathrm{~m}$ s.l.m. (Brichetti \& Fracasso, 2007). Nel PRM, la specie si è riprodotta nel 1981 con 1 coppia alla foce del fiume Ombrone (Romè \& Travison, 1982b). Successivamente, tentativo di riproduzione con costruzione del nido non portato a termine per abbandono del sito posto sotto un ponte: 1 coppia rinvenuta sino al 07/05/2000 in località Valentina, Talamone (GA e PG). In migrazione: 2 ind. dal 20 al 21/04/1991 nel Parco della Maremma (D. Occhiato in Arcamone \& Barbagli, 1995-1996).

\section{Cettiidae}

Usignolo di fiume Cettia cetti SB, M irr

In Europa, è nidificante con tendenza alla stabilità (Staneva \& Burfield, 2017). La specie si riproduce ai margini di zone umide di acqua dolce o salmastra, in luoghi con folta vegetazione ripariale erbacea e cespugliosa, fiumi a corso lento, fossi e canali, anche a ridosso di aree urbane risultando più diffusa sino a 300-400 m s.l.m.; in inverno si rileva un ampliamento di habitat con la frequentazione di orti, incolti e giardini anche lontani dalla presenza di corsi d'acqua (Brichetti \& Fracasso, 2010). Nel PRM, presenze monitorate durante tutto l'anno, grazie alla facile rilevabilità delle emissioni canore; le presenze vanno ad interessare in particolare il fiume Ombrone, le Macchiozze e l'intero reticolo idraulico caratterizzato da fossi e canali, purchè con adeguate caratteristiche ecologiche (PG; GA). La popolazione è verosimilmente costituita da individui sedentari a cui in inverno si aggiungono contingenti probabilmente in dispersione da aree limitrofe. Nel corso dell'intero anno 2013 rilevata la presenza stabile di 1-2 individui all'interno dell'ampia area caratterizzata unicamente da folta vegetazione con Juncus acutus, al confine tra la Tenuta di San Carlo e quella di Torre Trappola (PG). Nell'area protetta stimabile una popolazione minima di 30 coppie nidificanti. Comunissimo nella palude a canneto della Trappola il 28/09/1969 (Castellani, 1970).

\section{Aegithalidae}

\section{Codibugnolo Aegithalos caudatus SB, M irr}

In Europa, è nidificante con tendenza alla stabilità (Staneva \& Burfield, 2017). La riproduzione interessa vari ambienti boscati, preferibilmente di latifoglie, con sottobosco folto e alto, più diffuso sino a 1000-1200 m s.l.m.; in inverno utilizza gli stessi ambienti anche se appaiono maggiormente frequentate, tra le varie aree, le zone ripariali, umide, parchi e giardini (Brichetti \& Fracasso, 2011). Nel PRM, nel periodo 2004-2017, è specie localizzata e rilevata con basse densità durante la stagione riproduttiva, presente nei soprassuoli più freschi con latifoglie e boschetti misti, specialmente con ricco sottobosco, per esempio nelle zone 
di Malenotti in Alberese, così come Torre Bassa al Collecchio e alla Colombaia nella Tenuta di San Carlo (PG; GA). Segnalazioni giungono anche dalla Pineta Granducale dell'Uccellina (Pezzo, 2012). La popolazione è verosimilmente costituita da individui sedentari a cui in inverno si aggiungono contingenti probabilmente in dispersione da aree limitrofe. In questa stagione osservato facilmente in aggregazioni composte eccezionalmente da alcune decine di individui in erratismo: 24 ind. il 01/01/2010 nella pineta a Marina di Alberese (PG). Nell'area protetta stimabile una popolazione minima di 10 coppie nidificanti.

\section{Phylloscopidae}

\section{Luì verde Phylloscopus sibilatrix $\mathrm{M}$ reg}

In Europa, è nidificante stabile (Staneva \& Burfield, 2017). Durante le migrazioni rilevato nei querceti decidui e freschi, specialmente con piante disetanee, ma anche nei parchi, giardini ed in aree urbane; più facilmente rinvenuto al canto durante la migrazione primaverile, nelle fronde più sommitali degli alberi (Brichetti \& Fracasso, 2010). Nel PRM, contattato nei mesi di aprile e maggio nelle località San Rabano, Tenuta di San Carlo, Lasco di Alberese, e Itinerario "Faunistico Forestale" (GA; PG; F. Corsi). Massima concetrazione registrata: 9 ind. il 28/04/2000 nei pressi dell'abbazia di San Rabano (PG).

\section{Luì grosso Phylloscopus trochilus $\mathrm{M}$ reg}

In Europa, è nidificante in decremento (Staneva \& Burfield, 2017). Durante le migrazioni viene contattato in aree boscate ed in corrispondenza di alberi isolati, specialmente in fioritura se in primavera (Brichetti \& Fracasso, 2010). Nel PRM, è presente durante le migrazioni, verosimilmente quasi sempre nel solo mese di aprile quando ne viene udito il canto in varie zone della costa comprese tra Principina Mare e Scoglietto (GA; PG). La frequentazione dell'area protetta si protrae generalmente per un massimo di venti giorni circa.

\section{Luì piccolo Phylloscopus collybita $\mathrm{M}$ reg, $\mathrm{W}$ reg}

In Europa, è nidificante stabile (Staneva \& Burfield, 2017). Durante le migrazioni e con lo svernamento la specie è osservata nelle golene fluviali, zone umide, zone coltivate, macchia mediterranea, frutteti, parchi, giardini e orti urbani (Brichetti \& Fracasso, 2010). Nel PRM, negli anni, in inverno presenze regolari di singoli individui o piccoli gruppetti sparsi in tutta l'area protetta con un apparente maggior numero di contatti provenienti dalle zone con vegetazione arborea ed arbustiva poste ai margini del fiume Ombrone e della pineta tra Principina Mare e Collelungo (GA). Nella ricerca delle risorse alimentari risulta facilmente osservabile anche sulle fronde di alberi isolati e nelle zone coltivate, compresi gli oliveti ed i frutteti. 1 ind. della ssp. tristis il 31/01/2001 a Principina Mare (D. Occhiato in Arcamone \& Puglisi, 2006).

\section{Acrocephalidae}

Cannareccione Acrocephalus arundinaceus $\mathrm{M}$ reg, B

In Europa, è nidificante con trend non conosciuto (Staneva \& Burfield, 2017). In Italia, è nidificante "Quasi
Minacciato" (Peronace et al., 2012). Si riproduce in zone umide di varia natura e dimensione occupando fragmiteti puri o misti con altre specie vegetali igrofile; maschio rilevabile facilmente al canto, risulta maggiormente diffuso sino a 300-400 m s.l.m. (Brichetti \& Fracasso, 2010). Nel PRM, durante le migrazioni è specie piuttosto diffusa nei luoghi adatti, anche ai confini dell'area protetta, a volte in canneti di dimensioni esigue (ad es. nei pressi dei canali limitrofi al Collettore Occidentale, Talamone). Presenze regolari durante le stagioni di nidificazione nell'area delle Macchiozze, lungo il fiume Ombrone, nel canale Essiccatore e nei collettori adiacenti. Tra le più recenti: 1 ind. in canto il 31/05/2015 nel canale Essiccatore nei pressi della foce del fiume Ombrone (PG); 1 ind. in canto il 20/05/2016 alla Mastrellaia, lungo il fiume Ombrone (PG). A nord, nel comprensorio della Palude della Trappola, la specie risulta contattata nel fosso Razzo e nei canali limitrofi, ad es. 1 ind. in canto il 03/06/2012 lungo il Fosso dei Molini (F. Farsi e PG). Nell'area protetta stimabile una popolazione minima di 15 coppie nidificanti. Comunissimo nella palude a canneto della Trappola il 28/09/1969 (Castellani, 1970)

\section{Forapaglie castagnolo Acrocephalus melanopogon M reg, $\mathrm{W}$ reg ?}

In Europa, è nidificante con trend non conosciuto (Staneva \& Burfield, 2017). È specie elusiva e solitaria durante tutto l'anno nonchè territoriale anche in parte dell'inverno con individui migratori e svernanti legati prevalentemente a formazioni di vegetazione palustre piuttosto densa e almeno parzialmente allagata; in inverno rilevata sino a circa $750 \mathrm{~m}$ s.l.m. (Brichetti \& Fracasso, 2010). Nel PRM, durante le migrazioni segnalazioni certe riferite al periodo compreso tra il 1986 e il 2005 con occasionali rinvenimenti invernali di 1-3 individui nei fragmiteti alla foce del fiume Ombrone così come in alcuni lembi di canneto presenti nelle Tenute di San Carlo e delle Femminelle (GA; F. Corsi; PG; G. Radi; SL; R. Gildi). 1 ind. nel novembre 2015 lungo il canale di Collelungo nei pressi delle Grotte, Alberese (C. Martelli). Nell'area protetta è forse specie invernale sottostimata (F. Corsi). Osservati alcuni individui il 28/09/1969 nella palude a canneto della Trappola (Castellani, 1970).

Forapaglie comune Acrocephalus schoenobaenus M reg? In Europa, è nidificante stabile (Staneva \& Burfield, 2017). Migrazione primaverile con transito regolare e consistente sulle piccole isole; in questi periodi di movimenti frequenta anche ambienti asciutti, lontani dall'acqua, come incolti e coltivi (Spina et al. 1993; Brichetti \& Fracasso, 2010). Nel PRM, raccolte scarse informazioni di presenza certa: l'attività di inanellamento, ad oggi poco condotta nell'area protetta, permetterebbe di produrre interessanti approfondimenti, anche sulla fenologia delle migrazioni. 1 ind. il 04/05/1997 a Campo al Pino, Alberese (GA); 1 ind. il 11/04/2002 alla foce del fiume Ombrone (GA).

Cannaiola comune Acrocephalus scirpaceus M reg, B

In Europa, è nidificante stabile (Staneva \& Burfield, 2017). Si riproduce in zone umide di varia natura e composizione, localmente in aree urbane, occupando fragmiteti 
variamente strutturati, densi e ben sviluppati verticalmente, puri o misti con altre specie igrofile erbacee e con maschi contattabili facilmente al canto, risultando maggiormente diffuso sino a 200-300 m s.l.m.; durante le migrazioni rilevato anche in ambienti asciutti e coltivati (Brichetti \& Fracasso, 2010). Nel PRM, presenze puntuali rispetto a Acrocephalus arundinaceus con segnalazioni di singoli individui in canto provenienti dalla rete secondaria di canali collettori posti lungo il confine dell'area protetta, verosimilmente nella sola zona di Alberese. Raro lungo il corso del fiume Ombrone. Nelle stagioni riproduttive 2000-2004 il Canale Essiccatore, nel tratto Magazzini-Macchiozze, occupato da un massimo di 3 coppie (PG). 1 ind. in canto il 11/05/2016 nel fragmiteto lungo il fosso che scorre nei pressi della strada per Marina di Alberese (PG). Stimabile una popolazione minima di 5-8 coppie nidificanti.

\section{Cannaiola verdognola Acrocephalus palustris A-1}

Accidentale. 1 ind. il 02/05/2003 alla Valentina, Talamone (L. Ruggieri in EBN-Italia). In epoca antecedente all'istituzione del PRM: alcuni individui il 28/09/1969 nella palude a canneto della Trappola (Castellani, 1970).

\section{Canapino comune Hippolais polyglotta $\mathrm{M}$ reg, B}

In Europa, è specie nidificante in incremento (Staneva $\&$ Burfield, 2017). Durante la riproduzione frequenta ambienti vari e strutturalmente piuttosto diversificati con ampie superfici erbacee e uno strato arbustivo denso ma discontinuo, ricco di specie spinose, preferendo esposizioni assolate e substrati asciutti, risultando più diffuso sino a 600-800 m s.l.m. (Brichetti \& Fracasso, 2010). Nel PRM, in periodo di nidificazione, le segnalazioni sono riferite a singoli individui in canto nei pressi di Torre Bassa, nelle zone di Poggio Bianco, Scoglietto e foce del fiume Ombrone (PG). Vengono quindi occupati incolti assolati, ginestreti con siepi, formazioni arboree degradate e piuttosto dense, perimetri di zone umide con alberi sparsi. Tra le più recenti osservazioni: 1 ind. il 12/05/2013 allo Scoglietto (GA); 1 ind. il 31/05/2015 alle Saline di San Paolo, nei pressi della foce del fiume Ombrone (PG). Stimabile una popolazione minima di 8-10 coppie nidificanti. Segnalazione di presenza invernale di Hippolais sp. con 2 ind. il 29/12/1982 nel "Parco Regionale della Maremma" (Bonazzi e Vigarani in Toso, 1983).

\section{Cisticolidae}

Beccamoschino Cisticola juncidis SB, M reg, W reg

In Europa, è nidificante stabile (Staneva \& Burfield, 2017). La specie, durante tutto l'anno, frequenta ambienti molto aperti e assolati, con copertura erbacea densa, sia all'interno che all'esterno di zone umide d'acqua dolce o salmastra, sia in zone prative ben drenate ed anche in aree urbane o periurbane purchè invase da erbe infestanti o incolti sufficientemente alti e folti; in periodo riproduttivo presenze diffuse sino a $500 \mathrm{~m}$ s.l.m. (Brichetti \& Fracasso, 2008). Nel PRM, osservato in canto anche in corrispondenza di coltivi erbacei o di aree erbose di modesta estensione così come alla base della cava dismessa in località Burraia, Alberese. Una frazione della popolazione è verosimilmente costituita da individui sedentari a cui si aggiungono in inverno contingenti impegnati perlopiù in locali spostamenti. Altre segnalazioni provengono, per esempio, dalle Macchiozze, Pianacce e dal perimetro del Canale Essiccatore in Alberese, così come dalle aree del Paduletto di Collelungo, del Tombolo e Tomboletto a Talamone (PG; GA; S. Celletti; I. Boschi). Nelle stagioni di nidificazione 2000-2004 censiti 23 territori su un totale di 91 punti di ascolto con una frequenza relativa pari a $2,74 \%$. Stimabile una popolazione minima di 40 coppie nidificanti.

\section{Sylviidae}

\section{Capinera Sylvia atricapilla SB, M reg, W reg}

In Europa, è nidificante in incremento (Staneva \& Burfield, 2017). La riproduzione della specie avviene in ambienti moderatamente umidi ed ombrosi con un minimo grado di copertura arborea ed uno strato arbustivo non troppo denso, ma relativamente ben sviluppato in altezza, anche in aree di ridotte dimensioni, e risultando più diffusa sino a 1200-1300 m s.l.m.; la gamma di ambienti utilizzati per lo svernamento e durante le migrazioni risulta più ampia includendo anche la vegetazione ripariale, gli oliveti e i fragmiteti (Brichetti \& Fracasso, 2010). Nel PRM, viene facilmente contattata tra le formazioni vegetali mediterranee con macchia bassa e leccete, mentre risulta con basse densità, se non del tutto assente, nelle pinete litoranee (Pezzo, 2012). Una frazione della popolazione è verosimilmente costituita da individui sedentari a cui si aggiungono in inverno contingenti migratori ed individui in dispersione. Nelle stagioni di nidificazione 2000-2004 censiti 28 territori su un totale di 91 punti di ascolto con una frequenza relativa pari a 3,34\%. Stimabile una popolazione minima di 300 coppie nidificanti.

\section{Beccafico Sylvia borin M reg}

In Europa, è nidificante con trend incerto (Staneva \& Burfield, 2017). Specie molto elusiva, durante le migrazioni frequenta i medi strati della vegetazione arbustiva e moderatamente boscati, evitando solo le formazioni forestali troppo chiuse; in questi periodi sembra essere più frequente sino a $700 \mathrm{~m}$ s.l.m. (Brichetti \& Fracasso, 2010). Migrazione primaverile con transito regolare e consistente sulle piccole isole tirreniche (Spina et al., 1993). Nel PRM, raccolte scarse informazioni per quanto a primavera sia presenza certa (GA; PG). L'attività di inanellamento, ad oggi poco condotta nell'area protetta, permetterebbe di produrre interessanti approfondimenti, anche sulla fenologia delle migrazioni.

\section{Bigia grossa occidentale Sylvia hortensis A-1}

Accidentale. Specie precedentemente non rinvenuta (Giovacchini, 2006). 1-3 ind. dal 25/06 al 11/08/2007 in "Alberese" (V. Visconti e S. Bassi in EBN-Italia). In Toscana, negli anni, segnalazioni sempre più scarse in periodo riproduttivo; in Maremma stazioni verosimilmente non più rioccupate stabilmente (Arcamone $\&$ Puglisi, 2006).

\section{Sterpazzola Sylvia communis M reg}

In Europa, è nidificante stabile (Staneva \& Burfield, 2017). Durante le migrazioni rilevata ove sia presente un minimo di copertura cespugliata ed ai margini di qualsiasi 
formazione arbustiva e boschiva (Brichetti \& Fracasso, 2010). Migrazione primaverile con transito abbastanza regolare sulle piccole isole tirreniche (Spina et al., 1993). Nel PRM, osservazioni limitate, forse anche sottostimate per la scarsa propensione degli individui in transito ad emettere il canto se non nelle ultime tappe della loro migrazione. L'area protetta, pur rappresentando alcuni degli habitat verosimilmente idonei all'interno dell'areale della specie, non raccoglie alcun dato di nidificazione certa (Farina \& Martelli, 1979; Sposimo, 1997a).

\section{Magnanina comune Sylvia undata SB, M reg?}

In Europa, è nidificante in decremento (Staneva \& Burfield, 2017). In Italia, è nidificante "Vulnerabile" (Peronace et al., 2012). In periodo riproduttivo viene rinvenuta nella macchia mediterranea costiera ed interna, normalmente sino a circa $500 \mathrm{~m}$ s.l.m., ove predilige tra le altre le formazioni miste di Cistus sp., Pistacia lentiscus e Rosmarinus officinalis; in inverno movimenti di erratismo locale di portata sconosciuta, comunque con possibile apporto di migratori (Brichetti \& Fracasso, 2010). Nel PRM, è specie contattata sul Poggio di Fontelunga, Talamone, in un'area a gariga sul crinale dei Monti dell'Uccellina: in questa zona, nel periodo 2000-2010, tra maggio e luglio rilevati al massimo 2 individui in canto (GA; PG). Precedenti segnalazioni riferite all'area compresa tra la foce del fiume Ombrone e la Pineta Granducale dell'Uccellina, nei mesi di maggio e giugno, oggi non confermate (Farina \& Martelli, 1979; Pezzo, 2012). In inverno, singoli individui o piccoli gruppi rinvenuti lungo la fascia costiera settentrionale, più spesso in corrispondenza della zona dunale con prevalenza di Juniperus oxycedrus subsp. macrocarpa, Myrtus communis e Rosmarinus officinalis, mettono in risalto movimenti erratici su base locale (Corsi, 1995b). Alcuni dati invernali: 1 ind. il 11/01/1989 a Principina Mare (PG); 4-5 ind. il 12/12/2003 a Principina Mare (D. Occhiato in EBN-Italia); 1 ind. il 03/02/2013 a Principina Mare (R. Gildi); 1 ind. il 28/12/2017 nella Palude della Trappola (C. Martelli e PG in GOM-List). Stimabile una popolazione minima di 3-4 coppie nidificanti.

\section{Sterpazzola di Sardegna Sylvia conspicillata A-3}

Accidentale. Specie precedentemente non riportata (Giovacchini, 2006). La prima indicazione di presenza fa riferimento al monitoraggio eseguito nel periodo 6 maggio-18 giugno 1979 nella zona compresa tra la foce del fiume Ombrone e la Pineta Granducale dell'Uccellina in Alberese (Farina \& Martelli, 1979). Una seconda per l'area protetta: 1 ind. il 25/04/2016 in richiami nella pineta della Tenuta di San Carlo (M. Dragonetti e LP in GOMList). Si segnala infine: 6 ind. il 08/06/2018 alla foce del fiume Ombrone (E. Arcamone su Ornitho.it).

\footnotetext{
Sterpazzolina comune/di Moltoni Sylvia cantillans/ subalpina $\mathrm{M}$ reg, $\mathrm{B}$

La specie è stata recentemente suddivisa in due specie separate (Svensson, 2013; cfr. Brambilla et al., 2008). In Europa, Sylvia cantillans e $S$. subalpina sono nidificanti in incremento (Staneva \& Burfield, 2017). Nonostante la revisione sistematica, alla luce delle attuali conoscenze sulla loro possibile distribuzione nel Paese e delle diffi-
}

coltà riscontrate nell'accertamento dell'identità specifica di ogni singola segnalazione raccolta, si preferisce fare ricorso a una trattazione unica. Solitaria, nei periodi delle migrazioni si rinviene in una vasta gamma di ambienti cespugliati, mentre con la riproduzione preferisce spazi alberati, secchi e soleggiati ed ancora, macchia mediterranea, gariga, incolti arbustivi e ginestreti, risultando maggiormente diffusa sino a circa 800-1000 m s.l.m. (Brichetti \& Fracasso, 2010). Migrazione primaverile con transito consistente e regolare sulle piccole isole (Spina et al., 1993). Nel PRM, rilevata regolarmente in canto durante la migrazione primaverile, specialmente ai margini di formazioni boschive associate ad incolti e siepi. Segnalazioni di nidificazione possibile, comunque limitate al settore centro meridionale dell'area protetta, più recentemente: 2 ind. in canto il 27/06/2001 a Cala di Forno (PG); 3 ind. in canto il 10/05/2003 tra l'Apparita e Torre delle Cannelle, Talamone (PG); 1 ind. in canto il 15/05/2005 a Cala di Forno (PG); 1 ind. in canto il 23/05/2011 al Romitorio, Alberese (GA); 1 ind. S. cantillans identificato come tale perché in canto il 21/05/2014 nei pressi di Poggio alla Murcia, Talamone (PG). 1 ind. in canto il 30/05/2018 alle Cannelle, Talamone (PG, F. Farsi e F. Sabatini). Stimabile una popolazione minima di 5-10 coppie nidificanti. Segnalazione di S. c. albistriata: 1 ind. il 29/08/2012 alla foce del fiume Ombrone (E. Babl su Ornitho.it).

\section{Occhiocotto Sylvia melanocephala SB, M reg}

In Europa, è nidificante stabile (Staneva \& Burfield, 2017). Durante tutto l'anno è specie rinvenuta facilmente in ambienti di macchia mediterranea con copertura vegetale molto variabile, margini di boschi, rimboschimenti con lo strato arbustivo sufficientemente sviluppato, margini di zone umide, giardini in aree urbane; durante la nidificazione maggiormente diffusa sino a 800 $\mathrm{m}$ s.l.m., in inverno anche in ambienti più aperti come gli oliveti (Brichetti \& Fracasso, 2010). Nel PRM, in periodo riproduttivo, è dominante con elevate densità nella pineta a Pinus pinea, specialmente con sottobosco ricco e ben strutturato; altrove piuttosto frequente in ambienti adatti, compresi quelli rocciosi prossimi al mare, tranne che, per esempio, nella macchia alta ed in corrispondenza della duna con prevalenza di Juniperus oxycedrus subsp. macrocarpa e Myrtus communis dove anche in inverno appare essere più scarso (Pezzo, 2012). Una frazione consistente della popolazione è verosimilmente costituita da individui sedentari ai quali più facilmente si aggiungono in inverno erratici e migratori a corto raggio. L'attività di inanellamento, ad oggi poco condotta nell'area protetta, permetterebbe di produrre approfondimenti, anche sulla fenologia delle migrazioni. Nelle stagioni di nidificazione 2000-2004 censiti 37 territori su un totale di 91 punti di ascolto con una frequenza relativa pari a $4,42 \%$. Stimabile una popolazione minima di 200 coppie nidificanti.

\section{Regulidae}

Fiorrancino Regulus ignicapilla $\mathrm{M}$ reg, W reg

In Europa, è nidificante stabile (Staneva \& Burfield, 2017). Fuori dal periodo della nidificazione la specie viene rilevata più facilmente in piccoli gruppi, anche con 
Regulus regulus, in ambienti con un minimo di copertura arborea o arbustiva (Brichetti \& Fracasso, 2008). Nel PRM, è specie migratrice e svernante regolare, mostrando preferenze per Quercus ilex e Quercus pubescens, più raramente associato a conifere e alle piante arbustive della macchia alta. In estate pare essere effettivamente assente dall'area protetta (Tellini Florenzano, 1997b).

\section{Regolo Regulus regulus $\mathrm{M}$ reg, $\mathrm{W}$ reg}

In Europa, è nidificante in decremento (Staneva \& Burfield, 2017). Durante le migrazioni e svernamento frequenta quasi ogni tipo di ambiente, con un minimo di copertura arborea o arbustiva, compresi gli ambienti agrari e quelli strettamente urbani, associandosi anche al meno comune Regulus ignicapilla (Brichetti \& Fracasso, 2008) (Fig. 49). Nel PRM, è specie migratrice e svernante regolare, mostrando preferenze per i boschi maturi di conifere: verosimilmente con alte densità nelle pinete costiere di Pinus pinea; in inverno occasionale nelle cipressete con Cupressus sempervirens.

\section{Troglodytidae}

Scricciolo Troglodytes troglodytes M reg, B irr, W reg In Europa, è nidificante stabile (Staneva \& Burfield, 2017). In periodo riproduttivo viene rilevato in zone fresche e ombrose collinari e montane, preferibilmente nelle vicinanze di corpi d'acqua con cespugli, alberi e massi sparsi, più diffuso tra 500-600 e 1900-2000 m s.l.m.; in migrazioni ed inverno utilizza qualsiasi tipologia ambientale con un minimo di copertura arboreo-arbustiva (Brichetti \& Fracasso, 2007). Nel PRM, in periodo riproduttivo segnalazioni irregolari circoscritte a limitatissime aree: 1 ind. in canto il 02/06/2001 nei pressi dei ruderi dell'abbazia di San Rabano a 310 m circa (PG); 1 ind. in allarme il 30/05/2018 alle Cannelle, Talamone (PG, F. Farsi e F. Sabatini). In altri momenti dell'anno è specie a distribuzione piuttosto ampia e facilmente contattabile: la fascia fitoclimatica del Lauretum raccoglie gli individui della zona costiera, in buona parte svernanti, i quali occupano, per esempio, anche canneti, seminativi, cavità naturali ed artificiali (Corsi, 1995c).

\section{Sittidae}

\section{Picchio muratore Sitta europaea A-2}

Accidentale. Specie precedentemente non rinvenuta (Giovacchini, 2006). Erratismi stagionali: 1 ind. il 02/01/2011 a Marina di Alberese (LP in GOM-List); 1 ind. il 28/01/2012 nella pineta a Marina di Alberese (S. Hueting su Ornitho.it).

\section{Tichodromidae}

Picchio muraiolo Tichodroma muraria $\mathrm{M}$ reg, W reg

In Europa, è nidificante con trend incerto (Staneva \& Burfield, 2017). Specie rupestre ed elusiva, anche durante le migrazioni e lo svernamento osservata più facilmente dalla pianura a circa $800-900 \mathrm{~m}$ s.l.m. su ruderi, manufatti, pareti ripide con frequenti tratti a diversa esposizione per la presenza di fessure, comunque con sufficiente disponibilità di superfici interessate da vegetazione soprattutto erbacea che facilita la presenza di invertebrati quale fonte di cibo (Brichetti \& Fracasso, 2011). Nel PRM, è specie regolarmente presente durante le stagioni invernali. Negli anni osservato esclusivamente con singoli individui sulle falesie ed altre pareti rocciose o manufatti dei Monti dell'Uccellina, in particolare a Cala di Forno,

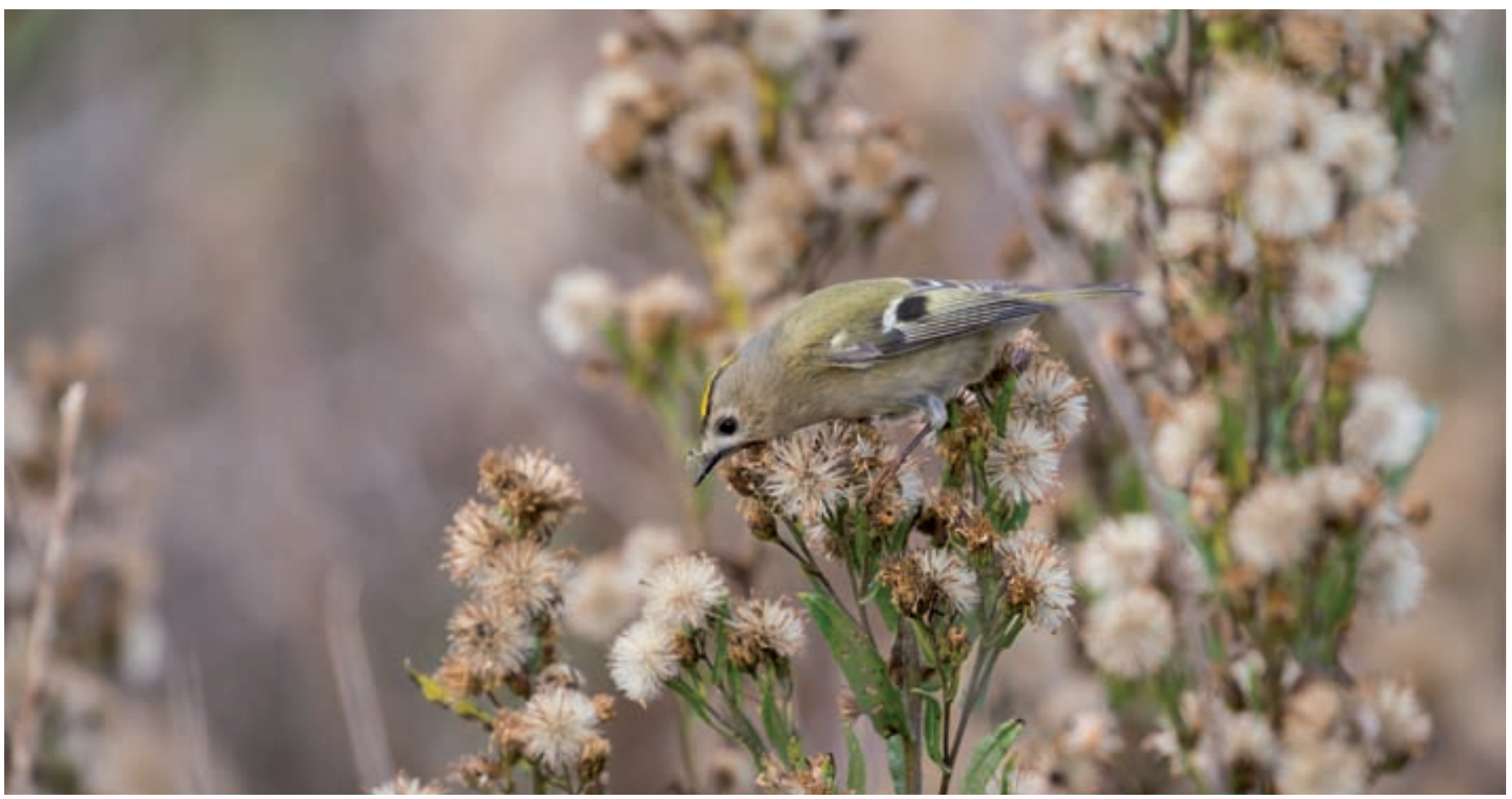

Fig. 49 - Regolo. / Goldcrest (Foto / Photo Giovanni Cappelli). 
Le Grotte-Scoglietto, sulle torri di Collelungo e Castel Marino. Altre località frequentate, a volte limitatamente esterne all'area protetta: 1 ind. il 16/12/2003 in spostamento sulle pareti della Rocca di Talamone (GA); 1 ind. il 11/01/2009 in esplorazione trofica sulla torre dell'abbazia di San Rabano (GA). Le osservazioni decorrono da novembre per proseguire sino a marzo, confermando precedenti indicazioni (Corazzi \& Travison, 1985). Dati estremi: 1 ind. il 02/11/2017 allo Scoglietto, Alberese (C. Martelli in GOM-List); 1 ind. il 08/03/1994 a Castel Marino (P. G. Talluri). Una segnalazione storica, riferita ad un periodo antecedente all'istituzione dell'area protetta: 1 ind. il 27/12/1972 in volo lungo la costa dei Monti dell’Uccellina (C. Castellani, ined.).

\section{Certhiidae}

Rampichino comune Certhia brachydactyla SB, M irr In Europa, è nidificante in incremento (Staneva \& Burfield, 2017). Specie strettamente arboricola e per lo più sedentaria, legata ad una disponibilità di grandi alberi vetusti e di legno morto, specialmente latifoglie a corteccia rugosa, in ambienti anche antropizzati come i parchi pubblici; normalmente presente al di sotto dei $1000 \mathrm{~m}$ s.l.m. (Brichetti \& Fracasso, 2011). Nel PRM, è rilevato all'interno del bosco di Quercus suber dell'itineriario "Faunistico-Forestale" in Alberese, così come a nord, nella Colombaia della Tenuta di San Carlo, caratterizzata da soprassuoli misti con prevalenza di Quercus pubescens e Pinus pinea (GA; PG). Altre segnalazioni provengono, in contesti simili, dal bosco d'alto fusto nei pressi di Poggio Spaccasasso in Alberese e dalla Valentina in Talamone, quest'ultima in inverno ad es. 1 ind. il 04/12/1996 (PG). Stimabile una popolazione minima di 20 coppie nidificanti.

\section{Sturnidae}

\section{Storno roseo Pastor roseus A-1}

Accidentale. 1 ind. il 18/12/1989 Palude della Trappola (Arcamone in Tellini Florenzano et al., 1997).

\section{Storno Sturnus vulgaris SB, M reg, W reg}

In Europa, è specie nidificante in decremento (Staneva \& Burfield, 2017). Notevole tendenza espansiva in Europa tra il XIX e XX secolo, negli ultimi 50 anni apparentemente invertita, necessitando in periodo riproduttivo di vaste superfici a copertura erbosa bassa per il foraggiamento e di cavità di varia natura purchè adatte alla nidificazione che avviene prevalentemente sino a 800 $\mathrm{m}$ s.l.m.; in autunno-inverno spiccatamente gregario con formazione di dormitori (Brichetti \& Fracasso, 2013). Nel PRM, nidificazione appurata per la prima volta in anni antecedenti all'istituzione dell'area protetta: 1 ind. con l'imbeccata il 18/06/1971 nella pineta della Trappola (Di Carlo, 1981). Successivamente, occorre attendere la stagione riproduttiva 1991 quando a maggio, lungo il corso del fiume Ombrone, in località Spolverino viene osservato un individuo impegnato nel portare l'imbeccata in un nido posto all'interno di una cavità su Populus alba, verosimilmente realizzata da Picus viridis (GA). Una frazione della popolazione è, con buone probabilità, costituita da individui sedentari ai quali si aggiungono in inverno erratici e migratori. Espansione moderata con colonizzazione delle aree di studio almeno sino alla fine degli anni ' 90 del secolo scorso occupando inizialmente i manufatti ed alberi posti in vicinanza di stalle e di rimesse per animali, cui è seguita una probabile stabilizzazione nel numero di coppie (GA; PG; I. Nerozzi). In autunno-inverno presenze diffuse in buona parte dell'area protetta in pianura, con contingenti difficilmente valutabili, ma legati alle superfici aperte come pascoli, incolti e coltivi alberati quali aree di alimentazione, anche in prossimità dei luoghi di raduno degli animali allevati all'aperto; senza alcuna ragione apparente, se non forse per un possibile disturbo procurato da predatori, registrata a volte l'occupazione dei roost per una sola parte dell'inverno (PG). Nella stagione 2015/16 il dormitorio posto su Pinus pinea, all'interno dell'azienda di Torre Trappola, ha indicativamente ospitato oltre 3000-4000 individui (PG e A. Picciau). Stimabile una popolazione minima di 50 coppie nidificanti.

\section{Storno splendente purpureo Lamprotornis purpureus} A-1

Occasionale nell'area di studio. In un recente passato risultava escluso dall'elenco delle specie dell'area protetta (Giovacchini, 2006). 1 ind. il 08/07/2000 alla foce del fiume Ombrone (GA). Identificazione supportata da documentazione fotografica, senza casi noti di riproduzione in natura ed esclusa dalla check-list dell'avifauna italiana (Baccetti et al., 2014; Brichetti \& Fracasso, 2015b).

\section{Turdidae}

\section{Merlo dal collare Turdus torquatus A-2}

Accidentale. 1 ind. nell'aprile 1986 a Vaccareccia, Alberese (Giovacchini, 1999). Si deve ora aggiungere: 1 ind. il 17/10/2007 in località Pian di Barca, Alberese (GA). In un recente passato la specie veniva segnalata di passo sull'Arcipelago Toscano, in concomitanza con eventi meteorologici avversi (Moltoni, 1975).

\section{Merlo Turdus merula SB, M reg, W reg}

In Europa, è nidificante in incremento (Staneva \& Burfield, 2017). Specie originariamente nidificante in soli ambienti boschivi maturi, freschi, anche con densa copertura arbustiva, purchè con disponibilità nel circondario di superfici aperte idonee alla ricerca del cibo, mostra una notevole plasticità ecologica con la colonizzazione di una vastissima gamma di habitat diversi, specialmente quelli modificati dalle attività umane, riproducendosi normalmente sotto i 900-1000 m s.l.m.; nel corso dell'anno presenta variazioni nell'uso dell'ambiente in risposta a cambiamenti soprattutto nella disponibilità di risorse alimentari e condizioni microclimatiche locali (Brichetti \& Fracasso, 2008). Nel PRM, è specie relativamente comune in periodo riproduttivo, ma solo in ambienti adatti. Risulta assente in aree sottoposte ad una intensa attività di pascolamento che comportino pesanti conseguenze a carico del sottobosco (Pezzo, 2012). Una frazione della popolazione è verosimilmente costituita da individui sedentari ai quali si aggiungono gli svernanti. L'attività di inanellamento, 
ad oggi poco condotta nell'area protetta, permetterebbe di produrre interessanti approfondimenti sulla fenologia della specie. Nei settori meridionali dei Monti dell'Uccellina, in particolare nei pressi di Talamone, la nidificazione (verosimilmente con basse densità) riguarda, ad esempio, le leccete collocate in aree più fresche rinvenute nei pressi di Poggio di Fontelunga con formazioni miste di Cistus sp., Pistacia lentiscus e Rosmarinus officinalis ed ancora le località Pancotto e Fonte Lepre, sempre con adeguata copertura fornita dal sottobosco; poco più a nord la specie è contattata regolarmente al Collecchio nei complessi forestali con latifoglie di Torre Bassa e poi, in Alberese, a Poggio Bianco e nella zona dell'Albatraia, così come a Principina Mare, con buone densità nella pineta della Tenuta di San Carlo e lungo il corso del fiume Ombrone (GA; PG). Individuo inanellato: 1 femmina catturata il $21 / 12 / 1983$ a Principina Mare, era stata inanellata il 25/04/1983 a Ventes Ragas, Lituania (Bendini \& Spina, 1990). Anomalia cromatica del piumaggio: 1 ind. parzialmente leucistico il 24/12/1998 a Principina Mare (GA e M. Lunardi). Nelle stagioni di nidificazione 2000-2004 censiti 9 territori su un totale di 91 punti di ascolto con una frequenza relativa pari a $1,07 \%$. Stimabile una popolazione minima di 40 coppie nidificanti.

\section{Cesena Turdus pilaris $\mathrm{M}$ irr, $\mathrm{W}$ irr}

In Europa, è nidificante in decremento (Staneva \& Burfield, 2017). Durante le migrazioni e l'inverno è specie maggiormente vincolata alle aperte superfici erbose, con alberi sparsi o ai margini dei boschi, spesso in prossimità di corpi idrici (Brichetti \& Fracasso, 2008). Nel PRM, presenze dettate specialmente da erratismi invernali, con osservazioni di singoli individui: 1 ind. il 05/12/1999 nella Tenuta di San Carlo (PG); 1 ind. il 06/01/2017 nella Palude della Trappola (M. Carabella, F. Pianezza e PG). Evidente tendenza a frequentare maggiormente i settori montuosi più interni.

\section{Tordo sassello Turdus iliacus $\mathrm{M}$ reg, W reg}

In Europa, è specie nidificante in decremento (Staneva $\&$ Burfield, 2017). In svernamento frequenta tra l'altro boschi misti di conifere e latifoglie, querceti vari, oliveti, vigneti, frutteti, macchia mediterranea e pinete litoranee (Brichetti \& Fracasso, 2008). Nel PRM, presenze di individui osservati anche in piccoli gruppi svernanti, con una evidente tendenza a frequentare maggiormente i settori montuosi più interni: massimo 5 ind. insieme il 07/01/1996 a Vergheria, Alberese (PG). Rilevato anche con altri Tur$d u s$ sp. ai margini dei più vasti soprassuoli ad aghifoglie nella zona nord dell'area protetta così come negli oliveti di Alberese. Dato il comportamento generalmente schivo e le tipiche fluttuazioni della specie nelle stagioni invernali caratterizzate da brevi presenze nell'area, è corretto attribuire la condizione di svernante solo ad una frazione dei contingenti (Sposimo, 1997b). Individuo inanellato: 1 ind. catturato il 17/12/1969 ad Alberese, era stato inanellato il 19/10/1968 a Ekeren, Belgio (Moltoni, 1973).

Tordo bottaccio Turdus philomelos $\mathrm{M}$ reg, $\mathrm{W}$ reg

In Europa, è nidificante in incremento (Staneva \& Burfield, 2017). Generalmente schivo, durante le migra- zioni e lo svernamento utilizza ambienti aperti e diversificati, dotati comunque di un minimo di copertura arboreoarbustiva, dal livello del mare alle zone basso montane, per esempio con oliveti, ginepreti, boschetti con arbusti bacciferi, pinete litoranee, aree boscate ai margini di zone umide (Brichetti \& Fracasso, 2008). Nel PRM, è specie contattata quasi esclusivamente con singoli individui. Diffuso ma non abbondante, è rilevato in tutti i settori dell'area protetta mostrando, rispetto a Turdus iliacus, una probabile maggiore propensione ad utilizzare stabilmente i boschi costieri, a cominciare dalla macchia mediterranea (Sposimo, 1997c). Individuo inanellato: 1 ind. catturato il 15/01/1975 ad Alberese, era stato inanellato il 02/07/1974 a Engure Lake, Lettonia (Corsi \& Giovacchini, 1995a).

\section{Tordela Turdus viscivorus $\mathrm{M}$ irr, W irr}

In Europa, è specie nidificante stabile (Staneva \& Burfield, 2017). Generalmente molto diffidente, durante le migrazioni e lo svernamento si osserva in una vasta gamma di ambienti, sempre piuttosto aperti ed adeguatamente alberati; ai contingenti locali impegnati in movimenti verticali si associano individui migratori provenienti dall'estero (Brichetti \& Fracasso, 2008). Nel PRM, le osservazioni fanno più facilmente riferimento a singoli individui rilevati in ambienti aperti, come i pascoli; limitate segnalazioni anche da coltivi arborei, come gli oliveti. Evidente tendenza a frequentare maggiormente i settori montuosi più interni. Negli anni, possibili presenze non rilevate per basse densità. Alcune osservazioni, durante le migrazioni: 12 ind. il 05/11/1995 alle Macchiozze (PG); 1 ind. il 16/02/2012 a Vergheria (GA). In inverno: 1 ind. il 24/12/2001 nella Tenuta di San Carlo (PG); 1 ind. il 22/01/2003 nei pressi della Villa Romana, Talamone (GA); oltre 1 ind. il 02/12/2016 alla foce del fiume Ombrone (A. Fabiani su Ornitho.it).

\section{Muscicapidae}

\section{Pigliamosche Muscicapa striata $\mathrm{M}$ reg, B}

In Europa, è specie nidificante stabile (Staneva \& Burfield, 2017). Generalmente solitario durante tutto l'anno e nettamente territoriale durante la riproduzione. In questo periodo utilizza formazioni forestali aperte e caratterizzate da strati medio-alti decisamente più sviluppati di quelli inferiori, insediandosi preferibilmente ai margini dei boschi, in coltivazioni moderatamente alberate, zone costiere rocciose, ma anche in contesti artificiali come manufatti ed abitazioni, dal livello del mare sino a poco oltre i $2000 \mathrm{~m}$ s.l.m.; presenza di M. s. tyrrhenica anche lungo la fascia costiera della Toscana (Brichetti \& Fracasso, 2008). Nel PRM, è diffuso specialmente nelle aree ecotonali di fondovalle e pianura, purchè nelle vicinanze di soprassuoli provvisti di adeguata copertura negli strati superiori, risultando oggi assente laddove sussista una intensa attività di pascolamento (Farina \& Martelli, 1979; Pezzo, 2012). Occupazione negli anni di numerosi manufatti di varia natura, compresi i ruderi dell'abbazia di San Rabano, così come di alcune cavità rocciose poste nella zona della Grotta dello Scoglietto, Alberese (PG; GA). Stimabile una popolazione minima di 70 coppie nidificanti. 
Pettirosso Erithacus rubecula SB, M reg, W reg

In Europa, è specie nidificante in incremento (Staneva \& Burfield, 2017). In periodo riproduttivo rilevato negli ambienti forestali freschi, ricchi di sottobosco, moderatamente densi, adattandosi anche a quelli antropizzati, generalmente tra 400-500 e 1600-1700 m s.l.m.; nel resto dell'anno frequenta quasi ogni tipo di habitat purchè fornito di un minimo di copertura arboreo-arbustiva (Brichetti \& Fracasso, 2008). Nel PRM, è specie molto localizzata in periodo riproduttivo, rilevata nelle forre fresche ed umide associate a latifoglie e boschi di maggiore pregio, a volte ai più rari corsi d'acqua, comunque a quote comprese tra 60-380 m circa. Il clima subarido che infatti caratterizza anche questa parte di costa, risulta decisivo nel determinare una bassa densità della specie in questo periodo dell'anno (Dinetti, 1997a). Ne consegue che una irrilevante frazione della popolazione è, con buone probabilità, costituita da individui sedentari ai quali si aggiungono copiosi gli svernanti grazie anche a movimenti verticali. Sino a 3 individui contattati in canto nei mesi di maggio e giugno nei pressi di Cala di Forno, all'Apparita, Fonte delle Monache, Poggio Raso e Cannelle, tutti tra il Collecchio e Talamone, così come tra Pian delle Cavalle e Poggio Alto ed al Condotto, in Alberese (GA; PG). In particolare, tra il 1990 e il 2007 rilevata l'occupazione a giugno per almeno 4 anni dell'area dell'Apparita (GA; PG; I. Nerozzi). Riproduzione accertata alle Cannelle: 1 giovane il 28/06/2002 nei pressi del Fosso della Campana (PG). In inverno è comune in tutta l'area protetta. In autunno-inverno si riscontrano indicativamente alte densità nelle formazioni vegetali a macchia bassa, negli oliveti, ai bordi delle paludi e nelle pinete a Pinus pinea, con i primi arrivi dei contingenti collocati, per esempio negli anni 1990-1994, tra il 2 e il 29 settembre (PG). Stimabile una popolazione minima di 5-7 coppie nidificanti. Individuo inanellato: 1 ind. trovato morto il 19/02/1994 ad Alberese, era stato inanellato il 22/09/1991 a Svenska Hogarna, Svezia (Archivio PRM).

Pettazzurro Luscinia svecica $\mathrm{M}$ irr ?, W irr

In Europa, è specie nidificante stabile (Staneva \& Burfield, 2017). Durante le migrazioni ed in inverno mostra una spiccata preferenza per le bordure ricche di vegetazione erbacea e arbustiva in prossimità dell'acqua, sia dolce sia salata; in questi periodi, riposo notturno entro formazioni elofitiche anche insieme ad altre specie di uccelli (Brichetti \& Fracasso, 2008). Nel PRM, segnalazioni irregolari per quanto probabilmente compaia nell'area protetta con numeri molto limitati. 1 maschio il 22/10/1992 nella Palude della Trappola (M. Mei); 1 maschio il 11/10/1998 alla Trappola (GA e PG); 1 ind. settembre 2012 alla Trappola (F. Pezzo). Segnalazione invernale: 1 ind. il 13/01/1984 nella Palude della Trappola (M. Pani, ined.).

\section{Usignolo Luscinia megarhynchos $\mathrm{M}$ reg, B}

In Europa, è specie nidificante in incremento (Staneva $\&$ Burfield, 2017). Si riproduce in ambienti boscati di latifoglie, con radure e folto sottobosco cespuglioso, più facilmente freschi e ombrosi collocati anche presso zone umide e corsi d'acqua; non disdegna tuttavia, aree rurali con siepi e boschetti così come parchi e giardini urbani. Maggiore diffusione sino a 500-600 m s.l.m. (Brichetti \& Fracasso, 2008). Nel PRM, distribuzione piuttosto ampia, principalmente in coincidenza con fasce di vegetazione arborea marginale e denso sottobosco, specialmente di fondovalle, cespuglieti a rovi, e con il corso del fiume Ombrone nei tratti maggiormente interessati dalla stratificazione dalla vegetazione arbustiva. Rilevato con basse densità per intensa attività di pascolamento nella Pineta Granducale dell'Uccellina (Pezzo, 2012). Interessante presenza riconducibile ad arrivi precoci: 1 ind. il 28/02/1992 in canto notturno nella zona del Pancotto, Talamone (PG). Nelle stagioni di nidificazione 2000-2004 censiti 8 individui in canto su un totale di 91 punti di ascolto con una frequenza relativa pari a $0,95 \%$. Stimabile una popolazione minima di 100 coppie nidificanti.

\section{Balia nera Ficedula hypoleuca $\mathrm{M}$ reg}

In Europa, è nidificante con trend non conosciuto (Staneva \& Burfield, 2017). In migrazioni rilevato in spazi aperti con cespugliati o singoli alberi sparsi, più facilmente in vicinanza a corpi idrici (Brichetti \& Fracasso, 2008). Nel PRM, ogni anno osservazioni di pochi individui, più facilmente nell'area compresa tra la foce del fiume Ombrone e la Palude della Trappola. Tra le più recenti: 1 ind. il 05/10/2008 nella Palude della Trappola (M. Dragonetti e PG); 1 ind. settembre 2010 alla foce del fiume Ombrone (A. Madella su Ornitho.it); minimo 5 ind. il 17/04/2015 alla foce del fiume Ombrone (ML). 1 ind. il 10/04/2016 alle Macchiozze (PG). Dato storico: osservato 1 maschio nella pineta della Tenuta di San Carlo il 11/05/1969 (Castellani, 1970).

\section{Balia dal collare Ficedula albicollis $\mathrm{M}$ irr}

In Europa, è nidificante in incremento (Staneva \& Burfield, 2017). Durante le migrazioni rinvenuto in ambienti ecotonali con radure boschive, meglio se caratterizzate dalla contemporanea presenza di soprassuoli maturi (Brichetti \& Fracasso, 2008). Nel PRM, osservazioni irregolari di singoli individui durante le migrazioni, anche in contesti utilizzati da Ficedula hypoleuca. Contingenti forse sottostimati. Recenti segnalazioni: 1 ind. il 16/04/1995 nei pressi di Alberese (GA); 1 ind. il 02/05/2009 a Spolverino, Alberese (GA); 1 ind. il 17/04/2015 alla foce del fiume Ombrone (ML su Ornitho.it).

\section{Codirosso spazzacamino Phoenicurus ochruros $\mathrm{M}$ reg,} W reg

In Europa, è nidificante in incremento (Staneva \& Burfield, 2017). Durante le migrazioni e lo svernamento frequenta preferibilmente zone aperte, anfratti dei muri, manufatti di altro genere, ambienti rocciosi vicini al mare e giardini (Brichetti \& Fracasso, 2008). Nel PRM, è specie diffusa in inverno con singoli individui in ambienti rocciosi, nei pressi degli insediamenti umani con caseggiati anche sparsi, ed ancora nei pascoli, incolti e nelle olivete, dove risulta verosimilmente abbondante. Nelle pinete costiere a Pinus pinea risulta segnalato con basse densità (Corsi, 1995d). Contingenti svernanti forse dovuti anche a movimenti verticali delle popolazioni montane nidificanti (Fig. 50). 


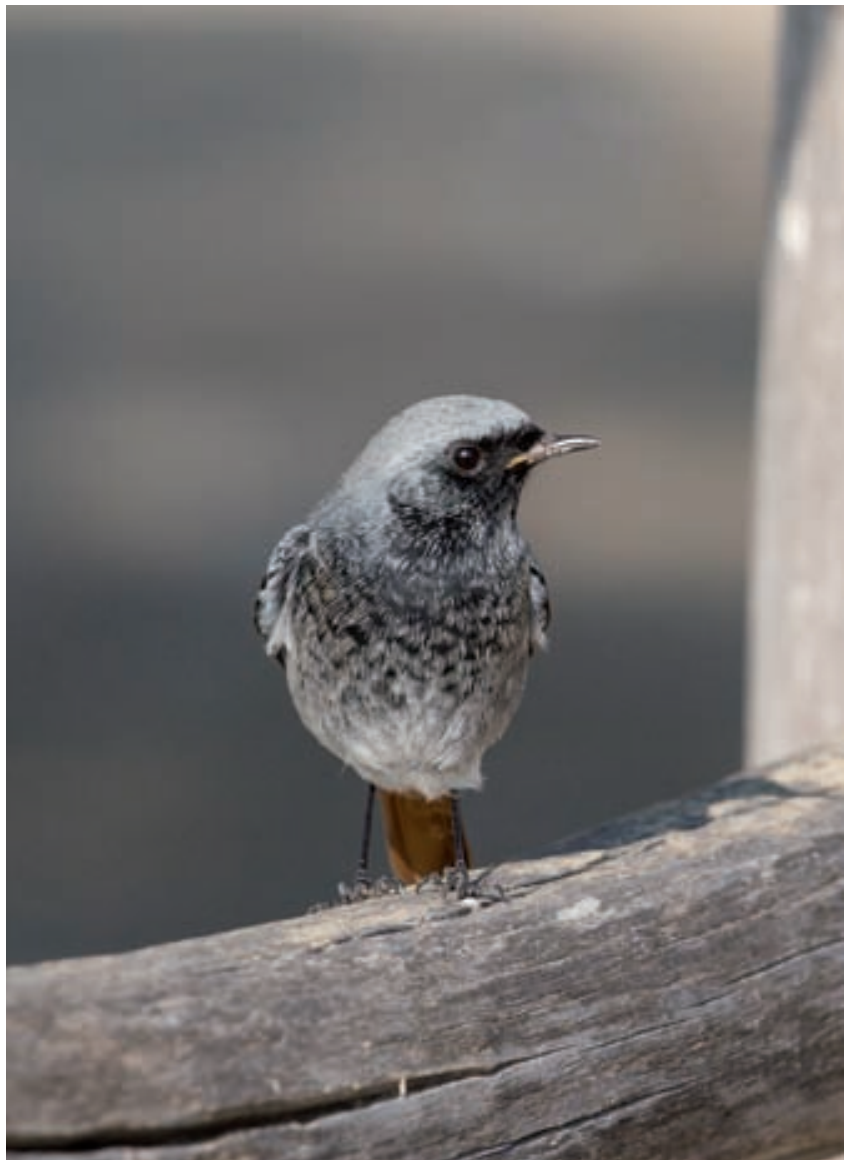

Fig. 50 - Codirosso spazzacamino. / Black Redstart (Foto / Photo Giovanni Cappelli).

Codirosso comune Phoenicurus phoenicurus M reg

In Europa, è nidificante in incremento (Staneva \& Burfield, 2017). Durante le migrazioni frequenta un ampio spettro di ambienti cespugliati e boscati, compresi i margini di zone coltivate (Brichetti \& Fracasso, 2008). Transito regolare a primavera sulle piccole isole tirreniche (Spina et al., 1993). Nel PRM, è specie presente regolarmente durante le migrazioni per quanto con contingenti difficilmente valutabili.

Passero solitario Monticola solitarius SB, M reg, W reg

In Europa, è nidificante con trend non conosciuto (Staneva \& Burfield, 2017). Specie molto schiva e territoriale durante tutto l'anno, più diffusa sino a 500-700 m s.l.m., in periodo riproduttivo è strettamente legata agli ambienti caldi e rocciosi caratterizzati da copertura erbacea poco sviluppata, e falesie rocciose estese in altezza, mostrando una buona capacità di colonizzare ambienti modificati dall'uomo anche all'interno di centri urbani; medesime preferenze ecologiche nel resto dell'anno (Brichetti \& Fracasso, 2008). Nel PRM, presenze durante tutto l'anno per quanto, sicuramente, con l'occupazione di alcuni siti soltanto in periodo invernale. Durante la stagione di nidificazione, la specie è stata osservata con maschi in comportamenti territoriali lungo i Monti dell'Uccellina, in aree caratterizzate dalla presenza di nicchie riparate in cavità rocciose su falesie, ad es. 1 ind. in canto il 10/03/2003 a Capo d'Uomo, Talamone (PG e GA); 1 ind. in volo di esibizione il 04/02/2007 a Capo d'Uomo, Talamone (PG). L'area in questione è occupata anche durante l'inverno (PG). Altra indicazione di presenza: 1 ind. in canto più a nord, sulle falesie tra Punta del Corvo e il Porticciolo il 02/04/1989 (PG e I. Nerozzi). In Alberese, nella zona compresa tra la Grotta della Fabbrica e Castel Marino risulta presente un altro sito riproduttivo, tra l'altro con femmine osservate unicamente in inverno: 1 ind. il 26/12/2001 nei pressi di Castel Marino (PG); 1 ind. il 01/12/2002 in cima ai ruderi di Castel Marino (PG). L'effettiva occupazione della cava di parete dismessa della Burraia, Alberese, con un maschio nella sola stagione invernale 2003/04, lascia propendere verso l'esistenza di movimenti migratori (Giovacchini, 2014). Stimabile una popolazione minima di 3-4 coppie nidificanti.

Stiaccino Saxicola rubetra M reg, W irr

In Europa, è nidificante in decremento (Staneva \& Burfield, 2017). Con le migrazioni e lo svernamento, frequenta una gamma di ambienti decisamente più ampia di quelli del periodo riproduttivo, sempre piuttosto aperti, più o meno erbosi e moderatamente cespugliati o alberati, anche i margini di zone umide (Brichetti \& Fracasso, 2008). Regolarità dei contingenti in transito sulle piccole isole tirreniche (Spina et al., 1993). Nel PRM, è specie presente in entrambi i periodi migratori, sicuramente con maggiore intensità a primavera (PG). Sono gli ambienti aperti di pianura, in special modo i pascoli e gli incolti, quelli nei quali si registrano anche interessanti presenze per l'area: poco oltre 20 ind. insieme il 07/09/2011 al Collecchio (PG). Recenti casi di presenze invernali: 1 ind. dicembre 2010-gennaio 2011 a Spergolaia, Alberese (M.G. Carpi e G. Natale in EBN-Italia); 1 ind. il 08/01/2012 alla foce del fiume Ombrone (J. Crisalli); 2 ind. il 22/12/2012 alla foce del fiume Ombrone (LP in GOM-List).

\section{Saltimpalo Saxicola rubicola M reg, B, W reg}

In Europa, è nidificante in decremento (Staneva \& Burfield, 2017). In Italia, è nidificante "Vulnerabile" (Peronace et al., 2012). Si riproduce in ambienti assolati, tendenzialmente asciutti e aperti, generalmente sino ad $800 \mathrm{~m}$ s.l.m., con elementi arbustivi-arborei sparsi e strato erbaceo di varia altezza; durante le migrazioni ed in inverno presente anche in ambiti maggiormente aperti con moderata vegetazione arbustiva (Brichetti \& Fracasso, 2008). Nel PRM, è specie verosimilmente in declino, specialmente come nidificante da dopo il 2010 circa, con presenze comunque ancora oggi regolari specialmente in aree agricole con pascoli o frutteti come quelle poste all'interno delle località Torre Trappola, Pianacce, Collecchio e Bonifica di Talamone (GA; PG; S. Celletti). In inverno è facilmente associato a zone umide, canali e aree moderatamente degradate, per quanto i contingenti presenti in questo periodo dell'anno possono risultare incrementati da individui provenienti dall'Europa centroorientale (Sposimo, 1997d). Nelle stagioni di nidificazione 2000-2004 censiti 5 individui in canto su un totale di 91 punti di ascolto con una frequenza relativa pari a $0,59 \%$. Stimabile una popolazione minima di 30 coppie nidificanti (Fig. 51). 


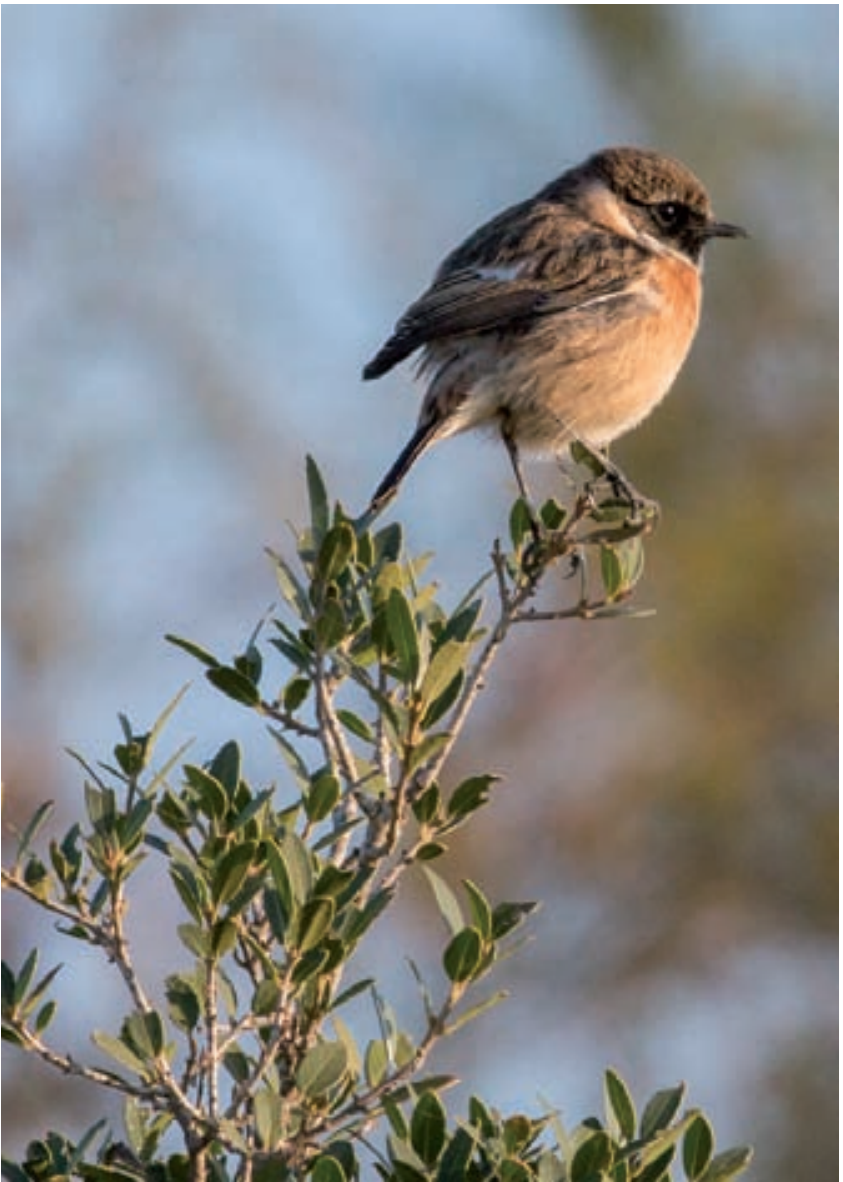

Fig. 51 - Saltimpalo. / Stonechat (Foto / Photo Giovanni Cappelli).

\section{Culbianco Oenanthe oenanthe $\mathrm{M}$ reg}

In Europa, è nidificante stabile (Staneva \& Burfield, 2017). Durante le migrazioni rilevato in ampi coltivi, pascoli asciutti e terreni arati (Brichetti \& Fracasso, 2008). Nel PRM, presenze regolari negli anni, specialmente di singoli individui rinvenuti in primavera in ambienti assolati e asciutti come pascoli, margini di terrapieni o fossi, nonché alla foce del fiume Ombrone. Verosimilmente meno frequente sui Monti dell'Uccellina in presenza di affioramenti rocciosi e cespugli sparsi. Osservazioni inusuali per l'area: 12 ind. il 10/04/1998 alla foce del fiume Ombrone (PG); 15 ind. il 27/04/2014 alla foce del fiume Ombrone (PG).

\section{Monachella del deserto Oenanthe deserti A-1}

Accidentale. Specie precedentemente non rinvenuta (Giovacchini, 2006). 1 maschio dal 02/12/2006 al 04/02/2007 svernante lungo la spiaggia di Principina Mare (R. Gildi, M. Azzolini, G. Chiancianesi, G. Conca, F. Gatti, F. Mantero, C. Marti, C. Musetti, R. Notaro, D. Occhiato e A. Voleri in EBN-Italia; G. Radi in GOMList; Fig. 52) (Mellone \& Sighele, 2007; Sighele \& Janni, 2009).

\section{Monachella Oenanthe hispanica M reg ? B irr}

In Europa, è nidificante stabile (Staneva \& Burfield, 2017). In Italia, è nidificante "In Pericolo" (Peronace

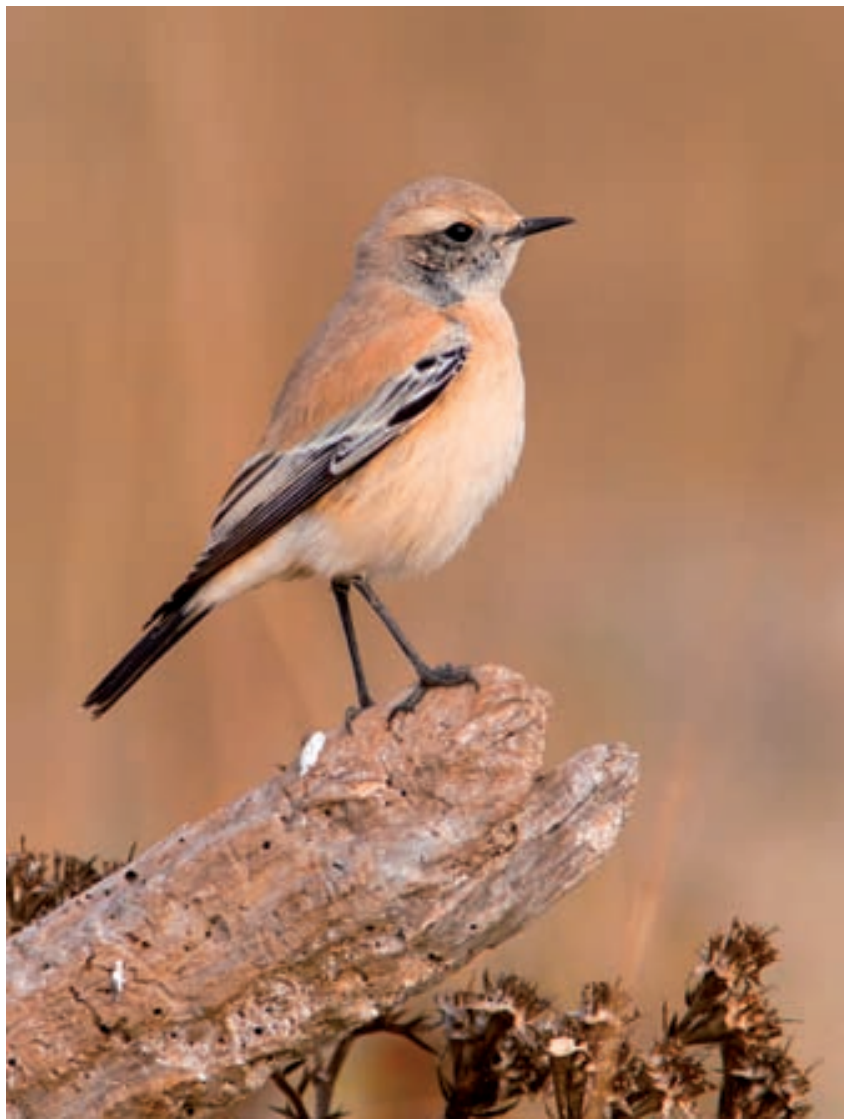

Fig. 52 - Monachella del deserto il 11/12/2006 sulla spiaggia di Principina Mare. / Desert Wheatear on the $11^{\text {th }}$ December 2006 on the Principina Mare beach (Foto / Photo Giacomo Radi).

et al., 2012). Tendenzialmente schiva, durante la riproduzione risulta poco comune al di sopra dei $1200 \mathrm{~m}$ s.l.m.: in questo periodo osservata in ambienti aperti di tipo mediterraneo o steppico, con componente arboreaarbustiva scarsa ed erbacea discontinua e con substrato sassoso, accidentato (Brichetti \& Fracasso, 2008). Nel PRM, rinvenuta specialmente con individui in migrazione in pascoli asciutti. Osservazioni per entrambe le varianti rinvenibili nel Paese ("gola scura" e "gola chiara"). La migrazione primaverile, per quanto forse non regolare nell'area protetta, risulta di maggiore intensità ad aprile. Massima concentrazione con 3-4 ind. insieme il 03/05/1996 alla foce del fiume Ombrone (M. Lausetti). Più scarse appaiono negli anni le osservazioni in autunno: 1 ind. il 17/09/1991 alla Trappola (PG); 1 femmina il 06/10/2008 alla foce del fiume Ombrone (G. Chiancianesi in EBN-Italia). In periodo riproduttivo segnalazioni irregolari di $O$. h. melanoleuca tra la Trappola e i Monti dell'Uccellina, anche prima dell'istituzione dell'area protetta: 1 coppia nidificante certa il 18/06/1971 alla Trappola (visto il maschio con l'imbeccata); 2 maschi il 22/06/1975 sempre alla Trappola (Di Carlo, 1977; 1981). Una recente osservazione di nidificazione probabile in ambiente roccioso caratterizzato da copertura erbacea irregolare: 1 maschio in canto almeno dal 19 al 23/06/2003 nei pressi dello Scoglietto, Alberese (PG). 


\section{Passeridae}

\section{Passera d'Italia Passer italiae SB}

In Europa, è specie nidificante in decremento (Staneva \& Burfield, 2017). In Italia, è nidificante "Vulnerabile" (Peronace et al., 2012). Commensale e sinantropico, si insedia anche in ambienti naturali e coltivi, con diffusione e densità in funzione generalmente della disponibilità di risorse alimentari nonché del grado di innevamento; più diffusa sino a 1300-1400 m s.l.m. (Brichetti \& Fracasso, 2013). Nel PRM, censite complessivamente circa 40 coppie nell'area di Spergolaia, Alberese, durante la stagione 2015 (PG); a nord del fiume Ombrone, nello stesso anno, censite almeno 20 coppie a Torre Trappola e nelle pertinenze (PG; F. Ferragamo Visconti e G. Visconti). In questo periodo dell'anno rilevata frequentemente l'utilizzazione di alcuni ponti stradali così come del Casello Idraulico alla foce del fiume Ombrone (GA; PG). Occasionale nidificazione nel 2010 presso la Casa dei Pinottolai, all'interno della Pineta Granducale dell'Uccellina (Pezzo, 2012). Una frazione importante della popolazione è sicuramente costituita da individui sedentari a cui forse si aggiungono nel corso dell'anno esemplari in dispersione e svernanti. Nella stagione più fredda, rinvenuta più facilmente in associazione con Fringilla coelebs, Serinus serinus e Chloris chloris (GA). Segnalati dormitori, specialmente autunno-invernali, ad esempio nelle zone di SpolverinoMacchiozze e negli spazi contigui ai canali di bonifica (GA). Stimabile una presenza minima di 400 coppie nidificanti. Nel periodo 2000-2004 rilevate 47 coppie su 91 punti di ascolto con una frequenza relativa pari al 5,61\%.

\section{Passera mattugia Passer montanus SB}

In Europa, è nidificante stabile (Staneva \& Burfield, 2017). In Italia, è nidificante "Vulnerabile" (Peronace et al., 2012). Specie rinvenibile nei coltivi di pianura e bassa collina, per quanto non disdegni situazioni sinantropiche anche nelle immediate vicinanze di abitazioni rurali e centri urbani, preferendo una certa copertura arborea, specialmente con boschetti, vigneti e frutteti e nidificando generalmente al di sotto dei $1000 \mathrm{~m}$ s.l.m. (Brichetti \& Fracasso, 2013). Nel PRM, durante tutto l'anno, è specie presente in aree aperte di fondovalle o moderatamente chiuse, per esempio, quelle interessate nelle vicinanze da recenti tagli forestali; diffusa, ma con densità piuttosto basse, in coltivi arborei specializzati, così come nelle stoppie cerealicole. Nidificante in cavità naturali o artificiali. Una frazione importante della popolazione è sicuramente costituita da individui sedentari a cui forse si aggiungono nel corso dell'anno esemplari in dispersione e svernanti. Nella stagione più fredda rinvenuta in associazione con Fringilla coelebs, Serinus serinus e Chloris chloris (GA). Stimabile una presenza minima di 100 coppie nidificanti. Nel periodo 2000-2004 rilevate 10 coppie su 91 punti di ascolto con una frequenza relativa pari al $1,19 \%$.

\section{Estrildidae}

\section{Bengalino comune Amandava amandava A-1}

Accidentale. 1 ind. ottobre 2001 alle Macchiozze(P. Sposimo e L. Colligiani, com. pers.). Nel Paese, è specie natura- lizzata; riproduzione regolare in alcuni settori della Toscana settentrionale (Andreotti et al., 2001; Baccetti et al., 2014).

\section{Prunellidae}

Sordone Prunella collaris M irr, W irr

In Europa, è nidificante con trend incerto (Staneva \& Burfield, 2017). Con le migrazioni ed in inverno frequenta, riunendosi spesso a gruppi, habitat rocciosi con una discreta copertura erbosa ed arbustiva, non raramente portandosi a quote prossime allo zero, così come ruderi, abitazioni isolate o piccoli agglomerati (Brichetti \& Fracasso, 2007). Nel PRM, presenze irregolari di 1-2 individui nelle zone comprese tra la foce del fiume Ombrone e Cala di Forno (Giovacchini, 1995m). Alcuni dati: 1 ind. il 23/01/1994 nell'area dei ruderi dell'abbazia di San Rabano (M. Gavazzi); 2 ind. il 01/03/1995 allo Scoglietto, Alberese (GA e I. Nerozzi); 1 ind. il 05/02/2001 a Castel Marino (GA); 1 ind. il 11/11/2008 al Sasso della Signora, Collecchio (GA e G. F. Martini); 1 ind. il 05/11/2010 a Cala di Forno (GA); 1 ind. il 18/12/2016 alla foce del fiume Ombrone (GA).

Passera scopaiola Prunella modularis M reg, W reg

In Europa, è nidificante stabile (Staneva \& Burfield, 2017). Durante le migrazioni ed in inverno, e per buona parte dell'anno, è specie schiva ed elusiva, frequentando una vasta gamma di ambienti cespugliati e con alte erbe (Brichetti \& Fracasso, 2007). Nel PRM, è specie sicuramente diffusa, specialmente in aree planiziali con radure, siepi e ai bordi della vegetazione ripariale del fiume Ombrone; segnalata anche nelle pinete purchè con adeguato sottobosco.

\section{Motacillidae}

\section{Cutrettola Motacilla flava $\mathrm{M}$ reg, B}

In Europa, è specie nidificante in decremento (Staneva \& Burfield, 2017). In Italia, è nidificante "Vulnerabile" (Peronace et al., 2012). Durante la riproduzione è presente in una vasta gamma di ambienti, molto aperti e pianeggianti, anche coltivati o prossimi a zone d'umide, caratterizzati da uno strato erbaceo piuttosto esteso, denso ma di varia altezza, con copertura arboreo-arbustiva rada, normalmente dal livello del mare fino a 100-200 m s.l.m. (Brichetti \& Fracasso, 2007). Nel PRM, la nidificazione interessa la sottospecie Motacilla flava cinereocapilla, con segnalazioni iregolari per $M$. $f$. feldegg nella Palude della Trappola (Foschi, 1976; Romè, 1980b; F. Pezzo). $M$. f. cinereocapilla è rilevata con basse densità, specialmente nei pascoli degli stagni delle Macchiozze: 0,6 coppie/10 ha (PG); nei salicornieti della Trappola-foce del fiume Ombrone osservazioni ancora nella stagione riproduttiva 2018 (PG); 1,1 coppie/10 ha nelle colture intensive a cereali ed orticole poste ai confini dell'area protetta, tra Alberese e Spergolaia (PG): la maggiore densità riproduttiva qui riscontrata potrebbe spiegarsi con le aumentate condizioni di sicurezza per la covata trovate in questi ambienti che compensano probabilmente i vantaggi legati alla prossimità dei siti riproduttivi all'acqua (Ferlini, 2015). Durante le migrazioni osservazioni ripetute di $M . f$. flava e $M . f$. feldegg (GA; M. Biondi; PG). Stimabile una presenza minima di 10 coppie nidificanti. 
Ballerina gialla Motacilla cinerea $\mathrm{M}$ reg, $\mathrm{W}$ reg

In Europa, è nidificante con trend incerto (Staneva \& Burfield, 2017). Durante le migrazioni ed in inverno, è specie poco gregaria; utilizza una più ampia gamma di habitat, tendenzialmente pianeggianti e aperti e, ancora una volta, preferibilmente in vicinanza di corpi idrici (Brichetti \& Fracasso, 2007). Nel PRM, presenze di migratori e svernanti: questi ultimi contingenti sono di difficile valutazione, tenendo anche conto della esistenza di individui verosimilmente impegnati in migrazioni verticali. Vengono quindi frequentati, più facilmente da singoli animali, margini di invasi artificiali, fossi e canali, prati semiallagati, il corso del fiume Ombrone e zone umide salmastre.

\section{Ballerina bianca Motacilla alba SB, M reg, W reg}

In Europa, è nidificante con trend incerto (Staneva \& Burfield, 2017). Specie tendenzialmente solitaria, più facilmente legata alla vicinanza dell'acqua ed eclettica quanto a preferenze ambientali frequentando, in particolare in periodo riproduttivo, zone umide di ogni dimensione, corsi dei fiumi, zone rurali nei pressi di cascinali, aree urbane, capannoni industriali ed edifici isolati, specialmente sino a $1500-1700 \mathrm{~m}$ s.l.m.; in inverno, rinvenuta facilmente nei campi lavorati, allevamenti zootecnici, margini di zone umide e centri abitati, anche con gruppi in attività trofica composti più comunemente da non oltre una decina di individui e in dormitori serali, questi ultimi spesso con cifre nettamente superiori al centinaio (Brichetti \& Fracasso, 2007). Nel PRM, è specie segnalata regolarmente, in particolare con singoli individui. Una frazione della popolazione potrebbe essere costituita da individui sedentari ai quali si aggiungono gli svernanti. Nidificante, per esempio, in zone rurali con abitazioni, stalle o cascinali (ad es. Podere Montesanto, Alberese, anno 1987; Podere Giovan Battista Vivarelli Colonna, Collecchio, anno 1998), lungo strade con canali o fossi e ponti (ad es. nei pressi del Podere Cava Zuccherina e fosso Perazzeta, Alberese, anno 2001), nei pascoli con coltivi alberati e manufatti sparsi (ad es. località Pianacce, Alberese, anno 2007) (GA; PG). In periodo invernale contattata nei campi lavorati, lungo strade, presso allevamenti zootecnici e ai margini di zone umide, con contingenti costituiti, come nel resto della Toscana, anche da individui provenienti dal nord Europa (Dinetti, 1997b). Stimabile una presenza minima di 30 coppie nidificanti. Nel periodo 2000-2004, rilevate 4 coppie su 91 punti di ascolto con una frequenza relativa pari al $0,47 \%$.

\section{Calandro maggiore Anthus richardi $\mathrm{M}$ irr, $\mathrm{W}$ irr}

Durante le migrazioni e lo svernamento frequenta zone costiere pianeggianti a livello del mare, con elevata copertura vegetazionale e ristagni d'acqua, senza disdegnare pascoli, margini di zone umide e coltivi (Brichetti \& Fracasso, 2007). Nel PRM, registrate 4 segnalazioni: 1 ind. rinvenuto morto nel gennaio 1987 in Alberese, al confine dell'area protetta (GA); 2 ind. il 14/10/2003 a Principina Mare (D. Occhiato in EBN-Italia); 1 ind. il 08/12/2005 alla foce del fiume Ombrone (L. Pardini e E. Occhiato in Mellone \& Sighele, 2006); 1 ind. il 08/02/2016 alla foce del fiume Ombrone (C. Martelli su Ornitho.it).

Calandro Anthus campestris $\mathrm{M}$ reg, $\mathrm{B}$

In Europa, è nidificante con trend sconosciuto (Staneva $\&$ Burfield, 2017). In periodo riproduttivo preferisce am- bienti aperti, di tipo steppico, tendenzialmente sabbiosi, caratterizzati da substrati asciutti e coperti, almeno in parte, da uno strato erbaceo molto basso con elementi arborei o arbusitivi molto radi, dal livello del mare sino a 1500-1600 $\mathrm{m}$ s.l.m.; con le migrazioni frequenta una più vasta gamma di habitat ma sempre molto aperti e secchi (Brichetti \& Fracasso, 2007). Nel PRM, segnalazioni nei pressi dei pascoli della foce del fiume Ombrone così come ai margini aperti della Palude della Trappola sino quasi a Principina Mare. Immediatamente fuori dall'area protetta frequentate anche le aree coltivate a cereali (per esempio: Podere Merano, Alberese). Osservato più facilmente con singoli individui, da maggio a settembre. Nel 2001, censite a maggio-giugno 2,5 coppie/10 ha nei pascoli ed incolti delle Macchiozze (PG). Negli anni 1998-2001, popolazione dell'area protetta valutata in $10-16 \mathrm{cp}$. (PG). Più recentemente, nel 2015 , stimate a giugno 1,2 coppie/10 ha nei pascoli asciutti della Tenuta di Torre Trappola (PG); nel 2017, da fine maggio ad inizio luglio complessivamente 7 maschi territoriali per l'intera area della Palude della Trappola sino alla foce del fiume Ombrone (PG e A. Picciau). Altre indicazioni di presenza, per es.: 1 ind. il 25/06/2004 nei coltivi erbacei della Bonifica di Talamone (PG); 1 ind. il 02/06/2013 nei pressi del Podere Ernesta, Talamone (PG). Interessante concentrazione: almeno 20 ind. insieme il 30/08/2012 alla foce del fiume Ombrone (ML su Ornitho.it). Stimabile una presenza minima di 6-7 coppie nidificanti. Nel periodo 2000-2004 rilevati 8 maschi cantori su 91 punti di ascolto con una frequenza relativa pari al $0,95 \%$. Distribuzione dei siti in periodo riproduttivo in Fig. 53.

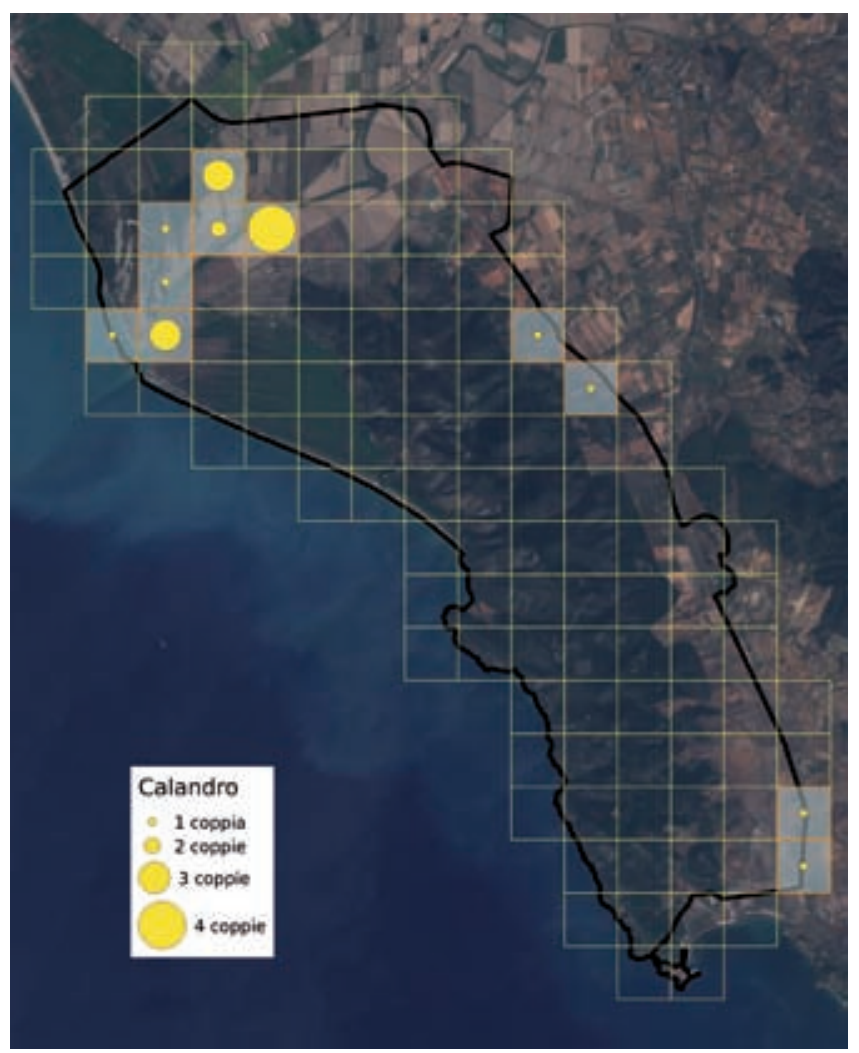

Fig. 53 - Siti di presenza di calandro in periodo riproduttivo. / Sites of presence of Tawny Pipit in breeding season (2001-2017). 


\section{Pispola Anthus pratensis $\mathrm{M}$ reg, $\mathrm{W}$ reg}

In Europa, è specie nidificante in decremento (Staneva $\&$ Burfield, 2017). Durante le migrazioni ed in inverno frequenta ambienti aperti ed erbosi come incolti, pascoli, canali, ma anche zone sabbiose, coltivate e margini di zone umide; generalmente gregaria con pochi individui o qualche decina, in gruppi poco coesi (Brichetti \& Fracasso, 2007). Nel PRM, è specie verosimilmente ben rappresentata durante la migrazione autunnale, con prime segnalazioni già a metà settembre: 2 ind. in richiamo il 11/09/2004 alle Macchiozze (GA). Comune e diffusa in inverno, rilevata anche in associazione con A. Spinoletta o Motacilla alba, occupa spazi aperti con coltivi ed incolti delle aree di fondovalle dei Monti dell'Uccellina, così come, tra Alberese e Principina Mare, utilizza estesamente i territori destinati all'intensa attività pascolativa e caratterizzati, seppur limitatamente, anche da prati umidi, acquitrini nonchè da margini di stagni (per esempio: Lasco di Alberese, Macchiozze). In questo periodo dell'anno, occupazione di tratti di spiaggia con duna, specialmente della zona di Principina Mare, anche con Anthus spinoletta (PG; GA). Per l'area protetta, segnalazioni riconducibili a gruppetti composti quasi sempre da un massimo di 5-6 individui (GA; PG).

\section{Prispolone Anthus trivialis $\mathrm{M}$ reg}

In Europa, è specie nidificante in decremento (Staneva \& Burfield, 2017). Schivo, in migrazioni frequenta spazi agrari e, in generale, più aperti rispetto alla stagione riproduttiva; nel complesso solitario o poco gregario (Brichetti \& Fracasso, 2007). In primavera contingenti in transito sulle piccole isole tirreniche (Spina et al., 1993). Nel PRM, segnalazioni occasionali alla foce del fiume Ombrone per quanto sia sicuramente specie regolare ma con uno scarso numero di individui in transito in entrambe le migrazioni: 1 ind. il 09/05/1999 alla foce del fiume Ombrone (GA); 3 ind. il 09/10/2016 alla foce del fiume Ombrone (G. Rossi su Ornitho.it).

\section{Pispola golarossa Anthus cervinus A-1}

Accidentale. Specie precedentemente non rinvenuta (Giovacchini, 2006). 1 ind. il 16/11/2012 nei pressi del Camping Talamone, Talamone (D. Gabriel su Ornitho.it).

\section{Spioncello Anthus spinoletta $\mathrm{M}$ reg, W reg}

In Europa, è nidificante stabile (Staneva \& Burfield, 2017). Generalmente schivo, fuori dal periodo riproduttivo si incontra singolarmente o in piccoli gruppi in ambienti caratterizzati per essere molto aperti su terreni umidi (Brichetti \& Fracasso, 2007). Nel PRM, rilevato in autunnoinverno, più facilmente con singoli individui alla foce del fiume Ombrone e nei salicornieti circostanti, così come ai margini della Palude della Trappola e in altre zone temporaneamente allagate, come le Macchiozze. Occupazione di tratti di spiaggia con duna, specialmente della zona di Principina Mare, a volte con Anthus pratensis (PG; GA).

\section{Fringillidae}

Fringuello Fringilla coelebs SB, M reg, W reg

In Europa, è nidificante stabile (Staneva \& Burfield, 2017). Durante la riproduzione è specie strettamente ter- ritoriale e solitaria, legata ad ogni tipo di ambiente boschivo, con una certa preferenza per le formazioni non troppo chiuse ma fresche e mature, con strato arbustivo rado e quello erbaceo basso, più diffusa sino a 1800-2000 $\mathrm{m}$ s.l.m.; nelle altre stagioni gregaria, con formazione di gruppi che alla fine dell'estate sono costituiti per lo più da giovani, in contesti arati o incolti con bassa vegetazione erbacea e scarsamente alberati (Brichetti \& Fracasso, 2013). Nel PRM, in periodo riproduttivo, rilevato in ambienti boschivi non troppo chiusi, risultando la specie più comune e con un buon grado di diffusione in tutta la Pineta Granducale dell'Uccellina caratterizzata da Pinus pinea (Pezzo, 2012). Nidificante anche in filari di alberi (GA). Una frazione della popolazione dovrebbe essere costituita da individui sedentari ai quali si aggiungono gli svernanti. Nei boschi, in inverno, è presente con singoli individui, mentre negli spazi aperti risulta molto più comune nei territori destinati ad attività pascolativa, così come in terreni incolti o lavorati. In questo momento dell'anno può essere osservato in dormitori comuni, gruppi vaganti o in alimentazione (ad es. Macchiozze, oltre 300 individui, inverno 2010/11, PG). In queste circostanze, rilevato anche con altri passeriformi. Stimabile una presenza minima di 100 coppie nidificanti. Nel periodo 2000-2004, rilevati 52 territori su 91 punti di ascolto con una frequenza relativa pari al 6,21\%. Individuo inanellato: 1 ind. catturato nel dicembre 1962 a Talamone, era stato inanellato il 08/10/1961 a Budakeszi, Ungheria (Moltoni, 1973).

\section{Peppola Fringilla montifringilla $\mathrm{M}$ irr ?, W irr?}

In Europa, è specie nidificante in decremento (Staneva $\&$ Burfield, 2017). Gregaria fuori dalla stagione riproduttiva, molto spesso associata a $F$. coelebs, viene rilevata in ambienti aperti alberati, grandi giardini, evidenziando un forte legame con Fagus sylvatica (Brichetti \& Fracasso, 2013). Nel PRM, segnalazioni irregolari, più frequenti lungo la costa, ma con numeri limitati; l'abitudine della specie a riunirsi in inverno con numerosi $F$. coelebs non facilita nell'accertamento delle effettive presenze. Registrate 3 segnalazioni: 1 maschio nel gennaio 1991 a Principina Mare (D. Occhiato, com. pers); 1 maschio il 21/12/2013 a Vaccareccia (G. Radi, M. Porciani, G. Bencini e PG); 1 ind. il 21/02/2016 nella Palude della Trappola (C. Castellani).

Frosone Coccothraustes coccothraustes $\mathrm{M}$ reg, W reg In Europa, è nidificante stabile (Staneva \& Burfield, 2017). Schivo e diffidente, durante l'inverno è specie meno strettamente boschiva, più ecclettica, che evita comunque gli spazi molto aperti, mantenendosi sempre in prossimità di elementi arborei (Brichetti \& Fracasso, 2013). Nel PRM, osservazioni regolari in autunno ed inverno, ma negli anni scarsamente sostenute da consistenti movimenti migratori e quasi sempre riferite a singoli individui rinvenuti prevalentemente in alimentazione ai margini di boscaglie, macchia mediterranea e negli oliveti (Porciani, 1995). In questo senso, contingenti moderatamente diffusi specialmente tra Alberese e Talamone (PG). In inverno, eccezionale osservazione per l'area: un gruppo di circa 40 ind. il 12/01/1994 alle Caprarecce, Talamone (GA). 1 maschio catturato nell'aprile 1880 a Ta- 
lamone, Orbetello, donato nel 1888 al National Museum of Natural History di Washington (catalogo delle cessioni reperti del Museo Zoologico La Specola, Firenze, F. Barbagli com. pers.).

\section{Ciuffolotto scarlatto Carpodacus erythrinus A-2}

Accidentale. 1 maschio in canto il 15/06/1988 alla foce del fiume Ombrone (E. Arcamone e E. Meschini in Arcamone \& Tellini, 1991-1992). Si deve ora aggiungere: 1 probabile maschio il 16/06/2016 a Principina Mare (S. Bassi in EBN-Italia).

Verdone Chloris chloris SB, M reg, W reg

In Europa, è nidificante stabile (Staneva \& Burfield, 2017). In Italia, è nidificante "Quasi Minacciato" (Peronace et al., 2012). In periodo di riproduzione frequenta, tra l'altro, spazi semiaperti con boschetti naturali o artificiali, pinete montane e costiere, vigneti, vivai, cimiteri e centri urbani (Brichetti \& Fracasso, 2013). Nel PRM, è specie a distribuzione discontinua; piuttosto scarsa come nidificante, limitata ai settori periferici dell'area protetta, come per esempio la zona della Tenuta delle Femminelle, nei pressi di Principina Mare, e quella della Valentina a Talamone (PG). Rinvenuto anche in soprassuoli posti in filari, specialmente di conifere (GA). Una frazione della popolazione è verosimilmente costituita da individui sedentari ai quali si aggiungono gli svernanti. In autunnoinverno appare piuttosto comune e diffuso negli spazi aperti intercalati da scarsi soprassuoli, più facilmente in associazione con altri fringillidi. Stimabile una presenza minima di 30 coppie nidificanti.

Fanello Linaria cannabina M reg, B irr ?, W reg

In Europa, è specie nidificante in decremento (Staneva $\&$ Burfield, 2017). In Italia, è nidificante "Quasi Minacciato" (Peronace et al., 2012). Durante la riproduzione è legato ad ambienti semiaperti ed eterogenei, con ampia disponibilità di elementi arbustivi intercalati a superfici dominate da erbe relativamente basse, ma produttrici di abbondanti semi, spaziando dai mosaici agrari, alle brughiere oppure macchie cespugliate e discontinue su versanti assolati, dal livello del mare sino circa $2300 \mathrm{~m}$ s.l.m.; in altri momenti dell'anno tende ad assumere un comportamento decisamente gregario, utilizzando habitat ancora più ampi con incolti, campi con stoppie e con vegetazione ruderale (Brichetti \& Fracasso, 2013). Nel PRM, rilevato in periodo riproduttivo in ambienti di gariga e macchia bassa, risultando presente sulla dorsale dei Monti dell'Uccellina, almeno tra Poggio di Fontelunga e Punta del Corvo. In questo periodo dell'anno: 3 ind. il 01/07/2001 in localtà Pancotto, Talamone (PG); 2 ind. in canto il 24/04/2007 al semaforo di Capo d'Uomo (PG). In inverno, presenze diffuse e, in alcuni anni, piuttosto copiose, spesso in aggregazione con altri fringillidi negli spazi aperti ove frequenta, per esempio, margini di boscaglie, oliveti, terreni incolti o lavorati, ruderi con circostanti affioramenti rocciosi. Stimabile una presenza minima di 5 coppie nidificanti.

Cardellino Carduelis carduelis SB, M reg, W reg In Europa, è nidificante stabile (Staneva \& Burfield,
2017). In Italia, è nidificante "Quasi Minacciato" (Peronace et al., 2012). In periodo riproduttivo, risulta legato a paesaggi semiaperti, diversificati, con disponibilità di alte erbe, anche ai margini di formazioni boschive di latifoglie o conifere di zone umide, in aree agricole o centri abitati, normalmente al di sotto dei $1500 \mathrm{~m}$ s.l.m.; in altri momenti dell'anno presente sopratutto negli incolti, ai bordi d'acqua e lungo vie di comunicazione (Brichetti \& Fracasso, 2013). Nel PRM, presenze comuni e diffuse durante tutto l'anno, con utilizzazioni di spazi aperti con incolti e margini di aree boscate, frutteti ed oliveti ed ancora in zone rurali con manufatti e vegetazione arborea sparsa o in formazioni lineari. Presente, con densità molto basse, nella Pineta Granducale dell'Uccellina (Pezzo, 2012). Una frazione della popolazione è verosimilmente costituita da individui sedentari ai quali si aggiungono gli svernanti. Nel corso dell'inverno concentrazioni di un certo interesse (ad es. Macchiozze, almeno 250 individui, inverno 2012/13, PG) in corrispondenza di incolti o residui di produzioni agricole con risorse trofiche abbondanti. Facilmente in associazione con altri fringillidi. Stimabile una presenza minima di 400 coppie nidificanti. Nel periodo 2000-2004, rilevati 64 territori su 91 punti di ascolto con una frequenza relativa pari al 7,64\%.

\section{Verzellino Serinus serinus SB, M reg, W reg}

In Europa, è specie nidificante in decremento (Staneva $\&$ Burfield, 2017). Durante la riproduzione frequenta ambienti soleggiati, semiaperti e strutturalmente diversificati per quanto riguarda le tipologie vegetazionali, con presenze regolari all'interno di centri urbani e comunque in vicinanza di ampie superfici erbose di varia altezza, sino alle formazioni boschive di latifoglie o conifere, più frequente al di sotto dei $1000 \mathrm{~m}$ s.l.m.; nelle altre stagioni è maggiormente eclettico, utilizzando facilmente superfici incolte ai margini delle città, sistemi viari ed aree agricole (Brichetti \& Fracasso, 2013). Nel PRM, tendenza ad essere poco comune durante la stagione riproduttiva, anche in prossimità di ampi spazi aperti con incolti e pascoli, ma non di conifere quando disposte in formazioni lineari $\mathrm{o}$ rade. Una frazione della popolazione è probabilmente costituita da individui sedentari ai quali si aggiungono gli svernanti. In inverno, è infatti specie rilevata con contingenti maggiori, ugualmente in ambienti aperti e soleggiati di pianura o collina, spesso in gruppi con Fringilla coelebs, Chloris chloris e Carduelis carduelis. Stimabile una presenza minima di 30 coppie nidificanti.

\section{Lucherino Spinus spinus M irr? W irr}

In Europa, è nidificante con trend fluttuante (Staneva \& Burfield, 2017). Specie decisamente arboricola: durante le migrazioni e lo svernamento osservate aggregazioni da decine ad alcune migliaia di individui, anche con altri fringillidi, e preferenza verso le formazioni di latifoglie produttrici di piccoli semi legate in particolare agli ambienti umidi ed ai corsi d'acqua; marcate fluttuazioni numeriche interannuali dei contingenti (Brichetti \& Fracasso, 2013). Nel PRM, presenze irregolari con limitati contingenti invernali, più recentemente: 2 ind. il 09/01/2006 in località Scoglietto, Alberese (PG); 3 ind. il 17/12/2017 alle Macchiozze, Alberese (PG). 


\section{Calcariidae}

Zigolo delle nevi Plectrophenax nivalis $\mathrm{M}$ irr, W irr

In Europa, è nidificante con trend sconosciuto (Staneva \& Burfield, 2017). Fuori dal periodo riproduttivo frequenta ambienti molto aperti, pianeggianti o poco inclinati e scarsamente inerbiti compresi i litorali sabbiosi, specialmente se ricchi di detriti spiaggiati (Brichetti \& Fracasso, 2015a). Nel PRM, segnalazioni in periodo invernale, raccolte unicamente lungo il litorale di Principina Mare, a cominciare dalla fine del secolo scorso: 1 ind. il 06/12/1999 (G. Premuda), con successive conferme sino al 08/01/2000 (M. Lausetti e R. Garavaglia; D. Occhiato in Arcamone \& Puglisi, 2006); nella successiva stagione: 2 ind. il 09/12/2000 (L. Ruggieri in Arcamone \& Puglisi, 2006). Infine: 1 ind. il 05/02/2003 (D. Occhiato in Arcamone \& Puglisi, 2006).

\section{Emberizidae}

Strillozzo Emberiza calandra $\mathrm{M}$ reg, B, W reg

In Europa, è specie nidificante stabile (Staneva \& Burfield, 2017). Si riproduce su terreni con substrato da umido a semiarido e con profilo ondulato, necessitando di spazi decisamente aperti, più facilmente incolti con vegetazione erbacea piuttosto densa e con alberelli isolati utilizzati come posatoi per il controllo del territorio e per le emissioni canore, dal livello del mare sino a poco oltre i 1500 m s.l.m.; durante le migrazioni ed in inverno, è specie decisamente gregaria a partire dalla fine del ciclo riproduttivo, con riposo notturno in cespugli e canneti, specialmente in aree aperte (Brichetti \& Fracasso, 2015a). Nel PRM, effettivi sicuramente avvantaggiati dalla esistenza di estese porzioni di terreno non sottoposte a coltivazioni intensive: censite 3,1 coppie/10 ha ha nei pascoli e negli incolti delle Macchiozze (PG). Presente ugualmente ai margini di zone umide (Palude della Trappola e Chiari del San Carlo), così come nei pascoli salmastri della Trappola dove sono state censite 1,0 coppie/10 ha (PG). Diffuso negli incolti dal Collecchio a Talamone, specialmente lungo canali o fossi (PG). Concentrazioni di un certo interesse in inverno: più recentemente, almeno 400 ind. in volo alla Trappola il 16/12/2016 (PG). Stimabile una presenza minima di 100 coppie nidificanti. Nel periodo 2000-2004, rilevati 43 territori su 91 punti di ascolto con una frequenza relativa pari al $7,64 \%$.

\section{Zigolo giallo Emberiza citrinella $\mathrm{M}$ irr, W irr}

In Europa, è specie nidificante in decremento (Staneva \& Burfield, 2017). Durante le migrazioni e lo svernamento, risulta legato a spazi molto aperti, sia naturali, quali i margini di zone umide stagnanti o correnti, sia artificiali coltivati, con vegetazione erbacea bassa o quasi assente, e incolti, non troppo lontano da alberi su cui rifugiarsi (Brichetti \& Fracasso, 2015a). Nel PRM, rinvenuto irregolarmente da novembre a febbraio con presenze, nel periodo 1986-2009 molto più spesso riconducibili all'area compresa tra la spiaggia di Principina Mare e la foce del Fiume Ombrone. Osservati quasi sempre singoli individui. Limitate eccezioni, con riferi- mento anche ai luoghi di svernamento: 2 maschi tra il 28/12/2000 e il 04/01/2001 nei pressi della Valentina, Talamone, con circostante presenza di vegetazione erbacea bassa (GA; PG); 2 ind. il 03/01/2009 sulla spiaggia di Principina Mare (SL e PG). Nel passato, inusuale segnalazione a primavera (Farina \& Martelli, 1979).

Zigolo golarossa Emberiza leucocephalos M irr, W irr

In Europa, è specie nidificante con trend sconosciuto (Staneva \& Burfield, 2017). Nei periodi di migrazione ed in quello di svernamento frequenta zone aperte, costiere e dell'interno, caratterizzate da incolti erbosi e cespugliosi, con buona copertura arbustiva e arborea come dune costiere, margini di zone umide, ampi alvei fluviali (Brichetti \& Fracasso, 2015a). Nel PRM, prime segnalazioni nell'inverno 1995/96, con 15 individui; massimo numero per l'area protetta registrato nella stagione di svernamento 1999/2000, con 21 individui sulla spiaggia a Principina Mare (Occhiato, 2003). Negli anni, rinvenuto irregolarmente tra l'ultima decade di novembre e la seconda di febbraio, sempre ed unicamente in habitat dunale, per un totale di almeno 9 stagioni sino all'inverno 2014/2015 per il quale si registra un massimo di 3 individui a gennaio (S. Benucci, F. Bodrero, R. Chaviano, A. Corso, D. Occhiato, E. Occhiato, P. M. Politi e F. Taube in EBN-Italia; G. Chiancianesi e C. Marti in Mellone \& Sighele, 2007; L. Vanni in Arcamone \& Puglisi, 2008; PG e SL; G. Radi; M. Lausetti e R. Garavaglia; Fig. 54). Osservato anche in associazione con E. cia e E. cirlus. Rilevate sensibili variazioni interannuali dei contingenti.

\section{Ibrido Zigolo giallo Emberiza citrinella x Zigolo gola- rossa Emberiza leucocephalos}

2 ind. il 17/01/2009 sulla spiaggia di Principina Mare (A. Corso in EBN-Italia).

\section{Zigolo muciatto Emberiza cia M irr? W irr}

In Europa, è specie nidificante in incremento (Staneva \& Burfield, 2017). In inverno, è rilevata in spazi più aperti e meno acclivi di quelli frequentati in periodo riproduttivo, compresi gli alvei fluviali, le garighe e spesso i coltivi (Brichetti \& Fracasso, 2015a). Nel PRM, segnalazioni limitate all'area compresa tra il Lasco di Alberese e Principina Mare, dove in inverno alcuni individui vengono anche osservati con E. cirlus, E. leucocephalos ed altri passeriformi, comunque in ambienti caratterizzati da incolti o pascoli con cespugli o dune costiere. Alcuni dati recenti: 4-5 ind. il 12/12/2003 a Principina Mare (D. Occhiato in EBN-Italia); 2 ind. il 22/01/2006 a Principina Mare (SL e PG); 1 ind. il 23/11/2012 al Lasco di Alberese (PG).

\section{Zigolo nero Emberiza cirlus SB}

In Europa, è nidificante stabile (Staneva \& Burfield, 2017). Solitario e fortemente territoriale durante tutto l'anno, in periodo riproduttivo rilevato facilmente in ambienti aperti e soleggiati, di varia natura e composizione, incolti o coltivati, purché provvisti di fasce cespugliate, macchioni di arbusti spinosi o filari alberati, più facilmente sino a 900-1000 m s.l.m.; in inverno in spazi relativa- 


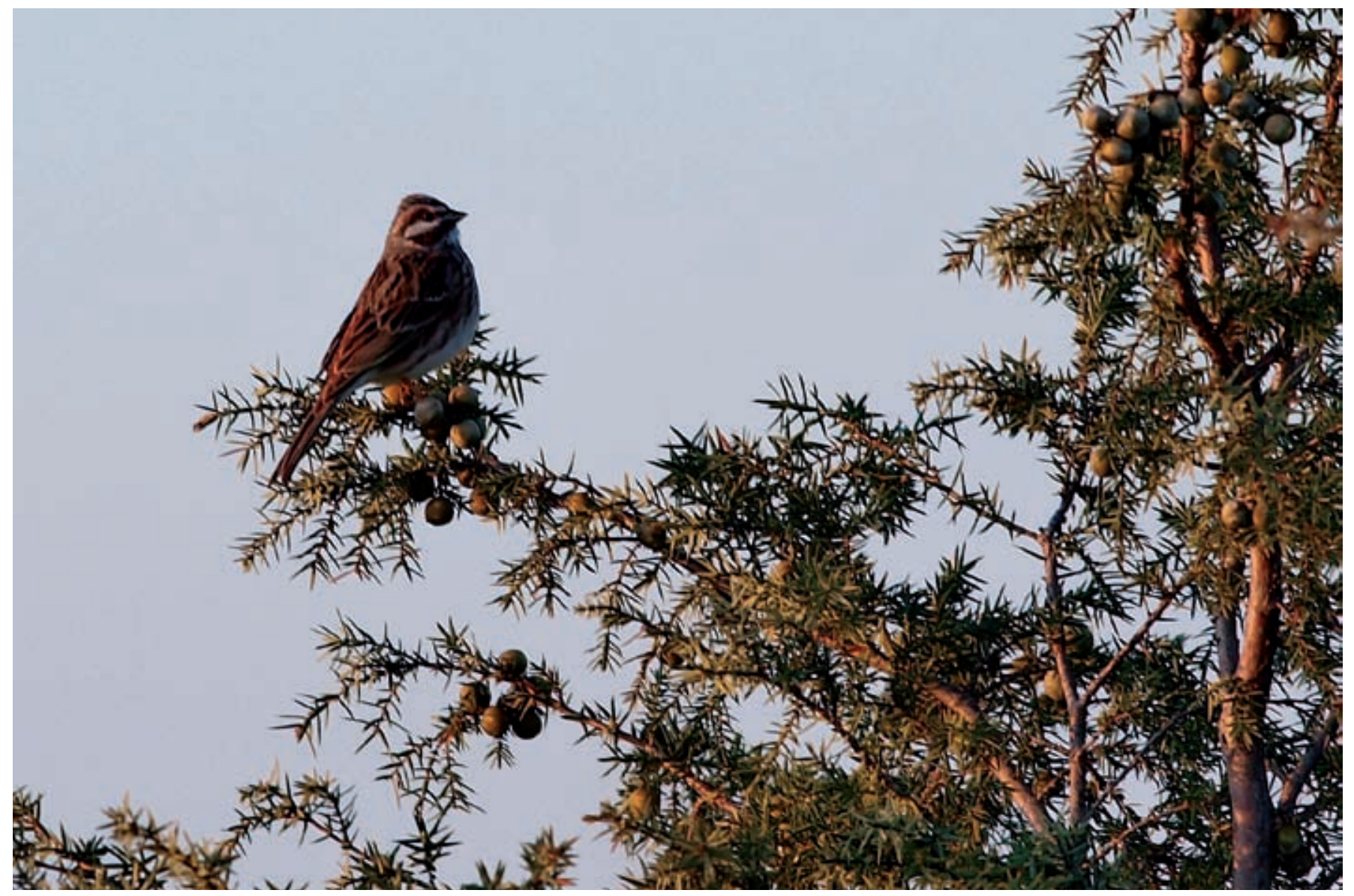

Fig. 54 - Zigolo golarossa il 03/01/2009 sulla spiaggia di Principina Mare. / Pine Bunting on the $3^{\text {rd }}$ January 2009 on the Principina Mare beach (Foto / Photo Stefano Laurenti).

mente più aperti, anche modificati dall'uomo, come campi di stoppie o lasciati ad incolti purché sempre in presenza di adeguata copertura arboreo-arbustiva (Brichetti \& Fracasso, 2015a). Nel PRM, nidifica in aree interessate da un forte legame tra superfici con vegetazione arbustiva e spazi aperti soleggiati, anche con colture arboree, portandosi a frequentare i margini di boschi a caducifoglie e conifere sino a formazioni erbacee di diversa natura, come quelle dominate da Ampelodesmos mauritanicus (Farina \& Martelli, 1979; Pezzo, 2012). Una frazione decisiva della popolazione è sicuramente costituita da individui sedentari a cui si aggiungono in inverno i dispersivi a breve raggio. In questa stagione, spesso numeroso in prati, pascoli, incolti, ambienti dunali, terreni lavorati, purché con adeguata copertura arbustiva nei pressi, anche in gruppo con altri passeriformi. Stimabile una presenza minima di 70 coppie nidificanti. Nel periodo 2000-2004 rilevati in periodo riproduttivo 24 territori su 91 punti di ascolto con una frequenza relativa pari al $2,86 \%$.

\section{Zigolo capinero Emberiza melanocephala $\mathrm{M}$ irr}

In Europa, è nidificante in decremento (Staneva \& Burfield, 2017). Specie completamente migratrice a lunga distanza; già alla fine della riproduzione e poi durante le migrazioni rilevata anche in gruppi con preferenze a frequentare campi coltivati e ambienti fittamente cespugliati (Brichetti \& Fracasso, 2015a). Nel PRM, verosimilmente migratrice irregolare, con avvistamenti forse riconducibili a nidificanti gravitanti in aree limitrofe laziali: 1 maschio il 25/09/1996 alla Trappola (PG).

Migliarino di palude Emberiza schoeniclus M reg, W reg, B ? (2001)

In Europa, è specie nidificante in decremento (Staneva \& Burfield, 2017). In Italia, è nidificante "Quasi Minacciato" (Peronace et al., 2012). Durante le migrazioni frequenta gli ambienti più vari, spesso lontano dall'acqua, tuttavia piuttosto aperti, erbosi e poco alberati, comprese le aree agricole, con gruppi di varia consistenza, generalmente al massimo di qualche decina di individui; nella Maremma Grossetana presenza di individui attribuibili a E. s. intermedia, con "becco grosso" (Brichetti \& Fracasso, 2015a). Nel passato, nel PRM veniva segnalato come nidificante in formazioni elofitiche lungo la costa della Maremma, compresa la Trappola-Bocca d'Ombrone (Baccetti \& Meschini, 1986; Brichetti \& Grattini, 2013). Recentemente, registrata una possibile nidificazione: 1 maschio il 18/05/2001 nel canale secondario bordato da canneti nei pressi delle Macchiozze (PG). Nel periodo di svernamento, viene rilevato anche fuori da contesti tipicamente palustri, più facilmente con singoli individui. Sono ugualmente utilizzati canali, zone cespugliate in contesti aperti e bacini di irrigazione purché con vegetazione di contorno al corpo idrico. 


\section{CONCLUSIONI}

Dall'istituzione del PRM, le specie di Uccelli rilevate al $31 / 12 / 2018$ sono complessivamente 295 , ovvero il $62,1 \%$ delle specie note per la Toscana ed il 52,5\% di quelle note per l'Italia, considerando nel computo Sylvia cantillans e $S$. subalpina come un'unica specie (Brichetti \& Fracasso, 2015b). I non passeriformi sono 189 (64\%), mentre i passeriformi sono $106(36 \%)$. Le specie per le quali sono state raccolte in tempi recenti (2008-2018) prove certe o indizi di probabile nidificazione sono 84 (28,4\% sul totale di quelle del PRM).

Tra le specie dell'avifauna del PRM:

- 88 sono di interesse comunitario presenti nell'Allegato I della Direttiva "Uccelli" 2009/147/CE;

- 33 sono nidificanti classificate come SPEC "Species of European Conservation Concern" (Staneva \& Burfield, 2017);

- 25 sono quelle con riproduzione certa o probabile inserite nella Lista Rossa 2011 degli Uccelli Nidificanti in Italia (Peronace et al., 2012).

Il PRM, con le zone umide "Bocca d'Ombrone" (cod. GR 0214) e "La Trappola" (cod. GR 0215), della più ampia Macrozona "Maremma Grossetana", ricade all'interno della qualifica di sito di importanza internazionale per la numerosità dei contingenti svernanti di Anser anser e Mareca penelope; l'area è anche considerata sito di importanza nazionale per Mareca strepera, Anas crecca, Anas acuta, Spatula clypeata, Ardea alba, Platalea leucorodia, Phoenicopterus roseus, Recurvirostra avosetta, Pluvialis apricaria, Calidris alpina, Vanellus vanellus, Gallinago gallinago, Numenius arquata, Tringa erythropus e Tringa totanus (Zenatello et al., 2014).
Nel corso dei prossimi anni è auspicabile che, con le nuove proposte di ricerca ornitologica, si possa dare prioritariamente seguito ad una serie di attività di studio e monitoraggi a medio-lungo termine, per esempio:

- inanellamento a scopo scientifico, con l'adesione al progetto MonITRing promosso da ISPRA attraverso il coordinamento del Centro Nazionale d'Inanellamento $(\mathrm{CNI})$;

- censimenti o stime di nidificanti di interesse comunitario nel rispetto della necessità di garantire una regolare verifica dei risultati raggiunti e legati alle conoscenze nella Rete Natura 2000 in applicazione alla Direttiva "Uccelli" 147/2009/CE (Nardelli et al., 2015), a cominciare dalle seguenti specie: Ixobrychus minutus, Burhinus oedicnemus, Caprimulgus europaeus, Lanius collurio, Lanius minor e Sylvia undata;

- censimenti o stime delle SPEC nidificanti in decremento (Staneva \& Burfield, 2017), alcune delle quali incluse anche nella precedente Direttiva: Ardea cinerea, Pernis apivorus, Fulica atra, Charadrius alexandrinus, Streptopelia turtur, Apus apus, Coracias garrulus, Alcedo atthis, Falco tinnunculus, Lanius minor, Lanius senator, Alauda arvensis, Galerida cristata, Hirundo rustica, Delichon urbicum, Sylvia undata, Sturnus vulgaris, Saxicola rubicola, Passer italiae, Motacilla flava, Linaria cannabina e Serinus serinus;

- promuovere attività di conservazione "attiva" tra $\mathrm{i}$ vari gestori delle zone umide/Siti Natura 2000, specialmente della costa medio tirrenica (ad esempio, Enti Parco, Regione Toscana, Uffici Territoriali Carabinieri per la Biodiversità ed associazioni ambientaliste) (Fig. 55).

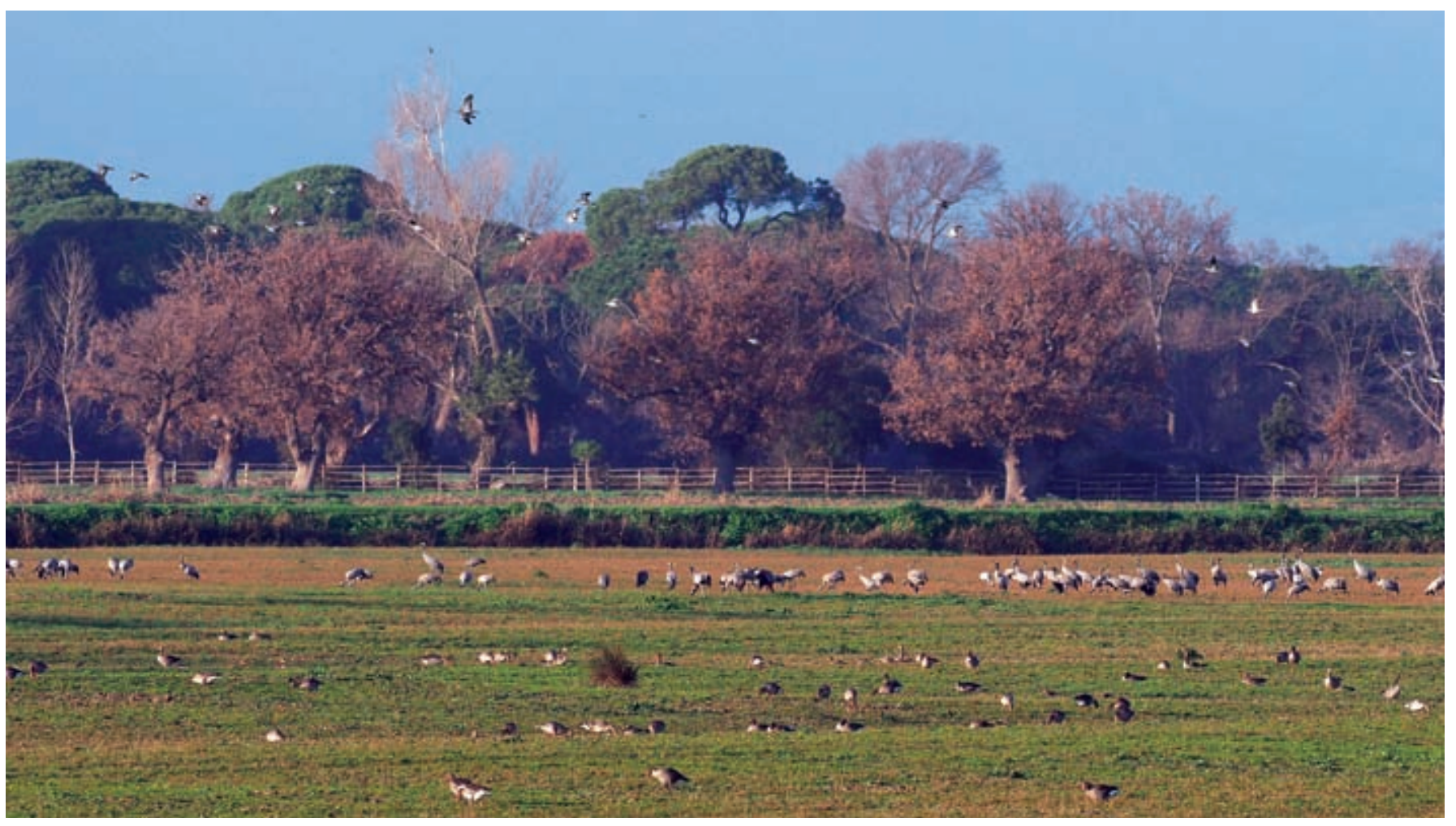

Fig. 55 - Pascolo di Torre Trappola con oche selvatiche, gru e colombelle / Pasture of Torre Trappola with Greylag Gooses, Cranes and Stock Doves (Foto / Photo Stefano Laurenti). 


\section{Ringraziamenti}

In primo luogo un sentito ringraziamento ai finanziatori che hanno contribuito a sostenere la stampa di questo fascicolo della prestigiosa Rivista italiana di Ornitologia - Research in Ornithology: in particolare, Tenuta Torre Trappola, Ente Parco Regionale della Maremma e Gruppo Ornitologico Maremmano - Studi Naturalistici "A. Ademollo". Grazie anche a Giuseppe Bogliani dell'Università degli Studi di Pavia nonché Direttore della Rivista, per avere accolto con entusiasmo la proposta di dedicare un fascicolo ad uno dei parchi regionali italiani "storici", così come a Francesco Pezzo che ha incoraggiato l'avvio del progetto.

Un pensiero affettuoso alla memoria della signora Fulvia Ferragamo Visconti che ha saputo catalizzare, con il marito avvocato Giuseppe Visconti, la mia personale e sincera stima, specialmente quando al gelo della tramontana invernale faceva da contrappunto il calore di un dialogo sorretto da sincero entusiasmo ed orgoglio nel contribuire a "custodire" la proprietà di Torre Trappola. Un ricordo personale rivolto all'amico di sempre, anch'egli prematuramente scomparso, Fausto Corsi che, da consigliere del Parco, ha messo a disposizione dell'Ente la sua competenza scientifica e il suo spirito di abnegazione.

Due anonimi referee sono stati di aiuto in fase di revisione del manoscritto: a loro giunga la mia personale gratitudine per l'importante lavoro realizzato.

Sono stati di insostituibile riferimento i fotografi: Giuseppe Anselmi, Marco Brandi, Doe Brendan, Giovanni Cappelli, Fabio Cianchi, Michelangelo Colli, Magnus Grylle, Michele Lamberti, Stefano Laurenti, Alberto Pastorelli, Giacomo Radi e Vincenzo Rizzo Pinna.

Per gli aspetti cartografici sono stati di supporto i colleghi Davide Melini e Cristiana Natali.

Oltre a coloro che, insieme al sottoscritto, hanno raccolto una significativa parte delle informazioni, sono altresì grato a tutti gli ornitologi ed i birdwatchers che in vario modo hanno fornito dati e notizie utili alla redazione del presente lavoro, anche attraverso la consultazione delle mailing list EBN-Italia, GOM-List, la piattaforma www.ornitho.it, così come il Centro Ornitologico Toscano che realizza per conto della Regione Toscana $\mathrm{i}$ censimenti IWC degli uccelli svernanti e l'Azienda delle Terre Regionali Toscane per la disponibilità che da sempre mostra agevolando l'accesso nelle aree di competenza, ed infine, con la speranza di non aver dimenticato qualcuno:

Fabio Acerbi, Mattia Altieri, Marco Anselmi, Mauro Apuleo, Emiliano Arcamone, Paolo Arrigucci, Maurizio Azzolini, Ethelbert Babl, Umberto Bambi, Thierry Bara, Fausto Barbagli, Silvio Bassi, Giovanni Bencini, Gianluca Bencivenga, Stefano Benucci, Alessandro Berardo, Livio Bernardini, Fabio Biagini, Massimo Biondi, Fabrizio Blangetti, Fabrizio Bodrero, Ugo Boldorini, Alessandro Boldrini, Laura Bonanno, Filippo Bonucci, Lucio Bordignon, Luca Boscain, Ilio Boschi, Erio Bosi, Marco Brandi, Doe Brendan, Pierandrea Brichetti, Luigi Buratta, Marco Cadanelli, Enrico Calvario, Alessandro Canci, Rita Capecchi, Alessandra Cappelli, Giovanni Cappelli, Mario Cappelli, Monica Carabella, Maria Gio- vanna Carpi, Francesco Carpita, Carlo Castellani, Mauro Carri, Paolo Cavallini, Guido Cazzola, Filippo Ceccolini, Guido Ceccolini, Stefano Celletti, Anna Cenerini, Ruben Chaviano, Sofia Chechi, Gianni Chiancianesi, Paolo Chietti, Angelo Chimenti, Fabio Cianchi, Michelangelo Colli, Linda Colligiani, Gianni Conca, Maurizio Corridori, Fausto Corsi, Andrea Corso, Joseph Crisalli, Antonio Delle Monache, Roy Dennis, Marco Dinetti, Ettore Di Masso, Francesco Di Murro, Marco Dragonetti, Sylvain Eichhoron, Fausto Fabbrizzi, Alessandro Fabiani, Fabrizio Farsi, Paolo Fastelli, Fabio Favali, Massimo Fedi, Riccardo Ferrari, Francesco Ferretti, Valerio Ferri, Riccardo Ferroni, Guido Tellini Florenzano, Dieter Gabriel, Matteo Gagliardone, Andrea Galimberti, Roberto Garavaglia, Francesco Gatti, Marco Gavazzi, Doriano Germani, Alessandro Gianni, Roberto Gildi, Fabrizio Giudici, Dimitri Giunchi, Magnus Grylle, Paul Harris, Steven Hueting, Alfredo Innocenti, Klaus Janich, Ottavio Janni, Alexis Lamek, Matteo Lausetti, Maurizio Lunardi, Roberto Macii, Marco Madeddu, Alessandro Madella, Annalisa Maggioni, Roberto Maistri, Giuseppe Manganelli, Fabrizio Mantero, Massimiliano Marcelli, Marco Marinelli, Paolo Marotto, Alessia Martelli, Claudio Martelli, Cristiana Marti, Gianfranco Martini, Marco Mei, Ugo Mellone, Enrico Meschini, Luciano Minucci, Riccardo Molajoli, Alessandro Montemaggiori, Flavio Monti, Marcello Moretti, Claudia Musetti, Riccardo Nardi, Giovanni Natale, Ivo Nerozzi, Laura Nigro, Rosario Notaro, Daniele Occhiato, Ernesto Occhiato, Ottavio Janni, Marcello Pacchiarotti, Giorgio Paesani, Marco Pani, Maria Cristina Paolini, Serena Paoloni, Roberto Papi, Lorenzo Pardini, Silvia Parenti, Bruno Parisotto, Menotti Passarella, Alberto Pastorelli, Fabio Perco, Brian Perroud, Francesco Perroud, Lorenzo Petruzzelli, Francesco Petretti, Francesco Pezzo, Federico Pianezza, Angela Picciau, Fabio Piccolo, Francesco Pieri, Paolo Maria Politi, Marco Porciani, Fulco Pratesi, Guido Premuda, Guido Prola, Alessio Quaglierini, Giacomo Radi, Roberto Ragazzini, Lorenzo Rigacci, Ettore Rigamonti, Alessio Rivola, Vincenzo Rizzo Pinna, Giuseppe Rossi, Luciano Ruggieri, Giampiero Sammuri, Andrea Santi, Stefano Sarrocco, Enzo Savo, E. Sbrana, Sergio Scebba, Gianluca Serra, Leonardo Siddi, Giancarlo Silveri, Marco Simoni, Riccardo Sirna, Paolo Sposimo, Giovanna Stellini, Massimo Taddei, Paola Grazia Talluri, Ivo Tanganelli, F. Taube, Luca Tinti, Laura Tonelli, Luca Tonini, Emanuele Valentini, Lorenzo Vanni, Domenico Verducci, Andrea Vezzani, Michele Viganò, Enzio Vigo, Fulvia Ferragamo Visconti, Giuseppe Visconti, Augusta Voleri, Enrico Zarri, Alessandro Zampieri.

Ai colleghi del Parco, con l'orgoglio di essere stato anch'io per lungo tempo guardiaparco di questa splendida area protetta. 


\section{BIBLIOGRAFIA}

AA.VV., 2000 - Parco Regionale della Maremma: Piano di gestione forestale. Studio RDM.

Allavena S., 1975a - Avvistamento di un Fenicottero (Phoenicopterus ruber antiquorum, Temminck) alla Trappola (Grosseto). Rivista Italiana di Ornitologia, 45: 228-229.

Allavena S., 1975b - Censimento di uccelli acquatici presenti nell'Italia centrale. Rivista Italiana di Ornitologia, 45: 252-262.

Andreella M., Bianchi E., Brecciaroli B. \& Pani F., 2008 - Attuazione della Direttiva Habitat e stato di conservazione di habitat e specie in Italia. Ministero dell'Ambiente e della Tutela del Territorio e del Mare, Direzione per la Protezione della Natura. Palombi Editore.

Andreotti A., Baccetti N., Perfetti A., Besa M., Genovesi P. \& Guberti V., 2001 - Mammiferi e Uccelli esotici in Italia: analisi del fenomeno, impatto sulla biodiversità e linee guida gestionali. Quaderni di Conservazione della Natura. 2. Ministero dell'Ambiente e Istituto Nazionale per la Fauna Selvatica.

Anselmi G., 1995a - Berta minore Puffinus yelkouan. In: Atlante degli Uccelli svernanti in provincia di Grosseto (inverni 1988/891993/94). Corsi F. \& Giovacchini P. (redattori). Amministrazione Provinciale di Grosseto, WWF Grosseto. Editrice Caletra: 33.

Anselmi G., 1995b - Beccapesci Sterna sandvicensis. In: Atlante degli Uccelli svernanti in provincia di Grosseto (inverni 1988/891993/94). Corsi F. \& Giovacchini P. (redattori). Amministrazione Provinciale di Grosseto, WWF Grosseto. Editrice Caletra: 146.

Anselmi G. \& Corsi F., 1992 - Caso di iperpredazione di Falco pellegrino Falco peregrinus su Sparviero Accipiter nisus. Picus, 18: 27.

Anselmi G., Corsi F. \& Parisotto B., 1989 - Segnalazione di nidificazione in nidi a tronchetto e all'interno di una ex cabina ENEL da parte di Ghiandaia marina e Assiolo. Picus, 15: 73-75.

Arcamone E., 1997a - Moretta tabaccata Aythya nyroca. In: Atlante degli uccelli nidificanti e svernanti in Toscana (1982-1992). Tellini Florenzano G., Arcamone E., Baccetti N., Meschini E. \& Sposimo P. (eds.). Quaderni del Museo di Storia Naturale di Livorno. Monografie, 1: 72-73.

Arcamone E., 1997b - Chiurlo piccolo Numenius phaeopus. In: Atlante degli uccelli nidificanti e svernanti in Toscana (1982-1992). Tellini Florenzano G., Arcamone E., Baccetti N., Meschini E. \& Sposimo P. (eds.). Quaderni del Museo di Storia Naturale di Livorno. Monografie, 1: 374 .

Arcamone E. (a cura di), 2005 - Nuovi avvistamenti. Avocetta, 29: 4450.

Arcamone E., Baccetti N. \& Serra L., 1994 - Limicoli svernanti nelle zone umide costiere della Toscana. Rivista italiana di Ornitologia, 64: 3-13.

Arcamone E. \& Barbagli F., 1995-1996 - Cronaca ornitologica toscana: 1990-1991. Quaderni del Museo di Storia Naturale di Livorno, 14: 79-109.

Arcamone E., Barbagli F. \& Meschini E., 2011 - L'avifauna del Parco naturale di Migliarino-San Rossore-Massaciuccoli dalle note di un ornitologo toscano degli inizi del Novecento. Edizioni ETS.

Arcamone E. \& Brichetti P. (eds.), 1995 - Nuovi avvistamenti. Avocetta, 19: 229-232.

Arcamone E. \& Brichetti P. (eds.), 1997 - Nuovi avvistamenti. Avocetta, 21: 220-227.

Arcamone E. \& Brichetti P. (eds.), 2001 - Nuovi avvistamenti. Avocetta, 25: 305-317.

Arcamone E., Dall'Antonia P. \& Puglisi L., 2007 - Lo svernamento degli uccelli acquatici in Toscana 1984-2006. Edizioni Regione Toscana.

Arcamone E. \& Massi A., 1987 - Lo svernamento delle oche (Gen. Anser) in Toscana. Quaderni del Museo di Storia Naturale di Livorno, 8: 131-137.

Arcamone E. \& Puglisi L., 2006 - Cronaca ornitologica toscana. Osservazioni relative agli anni 1992-2004. Alula, 13: 3-124.

Arcamone E. \& Puglisi L., 2008 - Cronaca ornitologica toscana. Osservazioni relative agli anni 2005-2007. Alula, 15: 3-121.

Arcamone E. \& Roselli A., 1997 - Rondone maggiore Apus melba. In: Atlante degli uccelli nidificanti e svernanti in Toscana (19821992). Tellini Florenzano G., Arcamone E., Baccetti N., Meschini E. \& Sposimo P. (eds.). Quaderni del Museo di Storia Naturale di Livorno. Monografie, 1: 184.
Arcamone E. \& Tellini G., 1985 - Cronaca ornitologica toscana: 19831984. Quaderni del Museo di Storia Naturale di Livorno, 6: 7994.

Arcamone E. \& Tellini G., 1986 - Cronaca ornitologica toscana: 1986. Quaderni del Museo di Storia Naturale di Livorno, 7: 105-118.

Arcamone E. \& Tellini G., 1987 - Cronaca ornitologica toscana: 1986. Quaderni del Museo di Storia Naturale di Livorno, 8: 139-154.

Arcamone E. \& Tellini G., 1988 - Cronaca ornitologica toscana: 1987. Quaderni del Museo di Storia Naturale di Livorno, 9: 75-90.

Arcamone E. \& Tellini G., 1991-1992 - Cronaca ornitologica toscana: 1988-1989. Quaderni del Museo di Storia Naturale di Livorno, 12 : 37-66.

Arrigoni P.V., 2003 - La flora vascolare del Parco della Maremma (Toscana, Italia centrale). Webbia, 58: 151-240.

Arrigoni P. V., Gellini R., Innamorati M., Lenzi Grillini C., Piussi P., Sartori G., Lovari S., Renzoni A. \& Sanesi G. (eds.), 1977 - Atti del convegno: Contributi alla conoscenza del paesaggio e dei problemi del Parco Naturale della Maremma. Informatore Botanico Italiano, 9: 141-216.

Arrigoni P.V., Nardi E. \& Raffaelli M., 1985 - La vegetazione del Parco Naturale della Maremma (Toscana). Università degli Studi di Firenze, Dipartimento Biologia Vegetale.

Arrigoni Degli Oddi E., 1929 - Ornitologia Italiana. Hoepli.

Baccetti N., 1989 - Lo svernamento del Cormorano in Italia. 7. Toscana. In: Lo svernamento del Cormorano in Italia. Baccetti N. (ed). Supplemento Ricerche di Biologia della Selvaggina, XV: 45-55.

Baccetti N., 1997a - Pittima reale Limosa limosa. In: Atlante degli uccelli nidificanti e svernanti in Toscana (1982-1992). Tellini Florenzano G., Arcamone E., Baccetti N., Meschini E. \& Sposimo P. (eds.). Quaderni del Museo di Storia Naturale di Livorno. Monografie, 1: 137.

Baccetti N., 1997b - Combattente Philomachus pugnax. In: Atlante degli uccelli nidificanti e svernanti in Toscana (1982-1992). Tellini Florenzano G., Arcamone E., Baccetti N., Meschini E. \& Sposimo P. (eds.). Quaderni del Museo di Storia Naturale di Livorno. Monografie, 1: 133.

Baccetti N., 2007 - I grandi uccelli erbivori delle praterie salmastre maremmane. In: Il Parco Regionale della Maremma e il suo territorio. Scapini F. \& Nardi M. (eds.). Pacini Editore.

Baccetti N., Cianchi F., Dall'Antonia P., De Faveri A. \& Serra L., 1994 - Nidificazione di Fenicottero, Phoenicopterus ruber, nella Laguna di Orbetello. Rivista Italiana di Ornitologia, 64: 86-87.

Baccetti N., Fracasso G. \& Gotti C., 2014 - La Lista CISO-COI degli uccelli italiani - Parte seconda: le specie naturalizzate (cat. C) e le categorie "di servizio" (cat. D, E, X). Avocetta, 38: 1-21.

Baccetti N. \& Massi A., 1992 - Airone bianco maggiore Egretta alba. In: Fauna d'Italia. XXIX. Aves. I. Brichetti P., De Franceschi P. \& Baccetti N. (eds.). Edizioni Calderini.

Baccetti N. \& Meschini E., 1986 - Confronto tra distribuzioni storiche e attuali di alcune specie in base ai dati del Progetto Atlante della Toscana. Rivista Italiana di Ornitologia, 56: 67-78.

Baccetti N., Panzarin L., Cianchi F., Puglisi L., Basso M. \& Arcamone E., 2008 - Two new Greater Flamingo (Phoenicopterus roseus) breeding sites in Italy. In: Flamingo. Bulletin of the IUC NSSC/ Wetlands International Flamingo Specialist Group. Childress B. Arengo F. \& Bechet A. (eds.). 16: 24-27. <https://www.wetlands org/wp-content/uploads/2015/11/Flamingo-Newsletter-16-2008. pdf $>$

Baccetti N. \& Roselli A., 1997 - Berta maggiore Calonectris diomedea. In: Atlante degli uccelli nidificanti e svernanti in Toscana (19821992). Tellini Florenzano G., Arcamone E., Baccetti N., Meschini E. \& Sposimo P. (eds.). Quaderni del Museo di Storia Naturale di Livorno. Monografie, 1: 30.

Baccetti N., Zenatello M., Della Toffola M., Alessandria G., Gola L., Piras W., La Gioia G., Roscelli F., Farioli A., Tinarelli R., Puglisi L., Arcamone E., Pezzo F. \& Mingozzi T., 2017 - Lo svernamento della gru Grus grus in Italia: analisi pluriennale (1992-2017) di un fenomeno in rapida evoluzione spazio-temporale. In: Riassunti del XIX Convegno italiano di Ornitologia (Torino 27 settembre-1 ottobre 2017). Fasano S. G. \& Rubolini D. (a cura di). Tichodroma, 6: 105 . 
Baghino L., Premuda G., Gustin M., Corso A., Mellone U. \& Cardelli C., 2007 - Exceptional wintering and spring migration of the booted eagle Hieraaetus pennatus in Italy in 2004 and 2005. Avocetta, 31: 47-52.

Barbieri F. \& Brichetti P., 1992 - Airone rosso Ardea purpurea. In: Fauna d'Italia. XXIX. Aves. I. Brichetti P., De Franceschi P. \& Baccetti N. (eds.). Edizioni Calderini.

Battaglia G. \& Sacchetti A., 1997 - Nitticora Nycticorax nycticorax. In: Atlante degli uccelli nidificanti e svernanti in Toscana (19821992). Tellini Florenzano G., Arcamone E., Baccetti N., Meschini E. \& Sposimo P. (eds.). Quaderni del Museo di Storia Naturale di Livorno. Monografie, 1: 42-43.

Bendini L. \& Spina F., 1983 - Bollettino dell'attività di inanellamento. Numero 2. Istituto Nazionale di Biologia della Selvaggina.

Bendini L. \& Spina F., 1990 - Bollettino dell'attività di inanellamento. Numero 3. Istituto Nazionale di Biologia della Selvaggina.

Benucci S., 1997 - Civetta Athene noctua. In: Atlante degli uccelli nidificanti e svernanti in Toscana (1982-1992). Tellini Florenzano G., Arcamone E., Baccetti N., Meschini E. \& Sposimo P. (eds.). Quaderni del Museo di Storia Naturale di Livorno. Monografie, 1: 175 .

Benussi E., 1992 - Astore Accipiter gentilis. In: Fauna d'Italia. XXIX. Aves. I. Brichetti P., De Franceschi P. \& Baccetti N. (eds.). Edizioni Calderini.

Bibby C.J., Burgess N.D., Hill D.A. \& Mustoe S.H., 2000 - Bird Census Techniques. Academic Press.

Biondi M., Pietrelli L., Guerrieri G., Corso A. \& Grussu M., 2000 - Il Corriere piccolo, Charadrius dubius, nell'Italia centrale e meridionale. Rivista Italiana di Ornitologia, 70: 97-114.

Bogliani G., 1985 - Distribuzione ed ecologia del Corvo, Corvus frugilegus, svernante in Italia. Rivista Italiana di Ornitologia, 55: 140150.

Brambilla M., Vitulano S., Spina S., Baccetti N., Gargallo G., Fabbri E., Guidali F. \& Randi E., 2008 - A molecular phylogeny of the Sylvia cantillans complex: Cryptic species within the Mediterranean basin. Molecular Phylogenetics and Evolution, 48: 461-472.

Brichetti P., 1980 - Distribuzione geografica degli uccelli nidificanti in Italia, Corsica e Isole Maltesi. 1. Parte introduttiva: Famiglie Podicipedidae, Procellariidae, Hydrobatidae. Natura Bresciana, 16: 82-158.

Brichetti P., 1982 - Distribuzione geografica degli uccelli nidificanti in Italia, Corsica e Isole Maltesi. 2. Famiglie Phalacrocoracidae, Ciconiidae, Treskiornithidae. Natura Bresciana, 19: 97-157.

Brichetti P., 1985 - Distribuzione geografica degli uccelli nidificanti in Italia, Corsica e Isole Maltesi. 4. Famiglie Ardeidae (generi Nycticorax, Ardeola, Egretta, Ardea). Natura Bresciana, 22: 41-102.

Brichetti P., 1992 - Tarabusino Ixobrychus minutus. In: Fauna d'Italia. XXIX. Aves. I. Brichetti P., De Franceschi P. \& Baccetti N. (eds.). Edizioni Calderini.

Brichetti P., De Franceschi P. \& Baccetti N. (eds.), 1992 - Fauna d'Italia. XXIX. Aves. I, Gaviidae-Phasianidae. Edizioni Calderini.

Brichetti P., Fasola M. \& Toso S., 1986 - Comitato di Omologazione delle specie accidentali. 4. Rivista Italiana di Ornitologia, 56: 245-246.

Brichetti P. \& Fracasso G., 2003-2013 - Ornitologia Italiana. Voll. 1-8. Alberto Perdisa Editore.

Brichetti P. \& Fracasso G., 2015a - Ornitologia Italiana. Vol. 9. Edizioni Belvedere.

Brichetti P. \& Fracasso G., 2015b - Check-list degli uccelli italiani aggiornata al 2014. Rivista Italiana di Ornitologia, 85: 31-50.

Brichetti P. \& Grattini N., 2010 - Distribuzione e trend delle popolazioni di Pendolino Remiz pendulinus nidificanti in Italia nel periodo 1980-2007. Picus, 36: 5-15.

Brichetti P. \& Grattini N., 2013 - Distribuzione, consistenza ed evoluzione delle popolazioni di Migliarino di palude, Emberiza schoeniclus, nidificanti in Italia nel periodo 1980-2010. Rivista Italiana di Ornitologia, 81: 97-109.

Brichetti P., Guyot I., Monbailliu X. \& Torre E., 1992 - Marangone dal ciuffo Phalacrocorax aristotelis. In: Fauna d'Italia. XXIX. Aves. I. Brichetti P., De Franceschi P. \& Baccetti N. (eds.). Edizioni Calderini.

Brichetti P. \& Occhiato D., 2005 - Commissione Ornitologica Italiana (COI). 19. Avocetta, 29: 93-107.
Brichetti P. \& Toso S. (eds.), 1989 - Nuovi avvistamenti. Avocetta, 13: 141-143.

Brogi S., 1896 - Catture di aquile. In: Notizie di caccia e note zoologiche. Bollettino del naturalista collettore allevatore coltivatore. Supplemento Rivista italiana di Scienze Naturali, 16: 67.

Brogi S., 1907 - Catture di specie rare e avventizie. Avicula, 109-110: 21-22.

Casini L. \& Genero F., 1992 - Moretta Aythya fuligula. In: Fauna d'Italia. XXIX. Aves. I. Brichetti P., De Franceschi P. \& Baccetti N. (eds.). Edizioni Calderini.

Castellani C., 1970 - Osservazioni ornitologiche del 1969 nelle paludi fra la foce dell'Ombrone e quella del Bruna (Grosseto). Rivista Italiana di Ornitologia, 40: 43-51.

Cattaneo G. \& Petretti F., 1992 - Biancone Circaetus gallicus. In: Fauna d'Italia. XXIX. Aves. I. Brichetti P., De Franceschi P. \& Baccetti N. (eds.). Edizioni Calderini.

Cauli F. \& Genero F., 2017 - Rapaci d'Italia. Edizioni Belvedere.

Cavallini P., 1992 - On the feeding behaviour of the Common Eiders in two coastal areas of Central Italy. Sitta, 6: 24-26.

Ceccolini G., Cenerini A., Baini M., Falchi V., Passalacqua L. \& Vignali S., 2013 - Restocking del Nibbio reale Milvus milvus in Toscana meridionale. In: Atti del II Convegno Italiano sui Rapaci diurni e notturni, Treviso 12-13 ottobre 2012. Mezzavilla F. \& Scarton F. (eds.). As.Fa.Ve., Quaderni Faunistici, 3: 11-20.

Chelini A., 1983 - Le zone umide nei comprensori di bonifica della Toscana, del Lazio, dell'Umbria e della Sardegna. Istituto di Tecnica e Propaganda Agraria.

Chelini A., 1984 - Le anatre selvatiche. Editoriale Olimpia.

Ciuffoletti Z. \& Guerrini G. (coordinamento di), 1989 - Il Parco della Maremma. Storia e Natura. Giunta Regionale Toscana. Marsilio Editori.

Colli M. \& Giovacchini P., 2016 - Osservazioni di una Gru Grus grus leucistica nel Parco Regionale della Maremma (Grosseto, Toscana). Picus, 42: 43-44.

Colombini I. \& Chelazzi L. 2007 - Presentazione dell'area: aspetti geografici, geomorfologici, idrografici e climatici. In: Il Parco Regionale della Maremma e il suo territorio. Scapini F. \& Nardi M. (eds.). Pacini Editore.

Corazzi A. \& Travison G., 1985 - Notizie ornitologiche dalla Toscana. Gli Uccelli d'Italia, 10: 73.

Corsi F., 1995a - Pittima reale Limosa limosa. In: Atlante degli Uccelli svernanti in provincia di Grosseto (inverni 1988/89-1993/94). Corsi F. \& Giovacchini P. (eds.). Amministrazione Provinciale di Grosseto, WWF Grosseto. Editrice Caletra: 125.

Corsi F., 1995b - Magnanina comune Sylvia undata. In: Atlante degli Uccelli svernanti in provincia di Grosseto (inverni 1988/891993/94). Corsi F. \& Giovacchini P. (eds.). Amministrazione Provinciale di Grosseto, WWF Grosseto. Editrice Caletra: 189.

Corsi F., 1995c - Scricciolo Troglodytes troglodytes. In: Atlante degli Uccelli svernanti in provincia di Grosseto (inverni 1988/891993/94). Corsi F. \& Giovacchini P. (eds.). Amministrazione Provinciale di Grosseto, WWF Grosseto. Editrice Caletra: 171.

Corsi F., 1995d - Codirosso spazzacamino Phoenicurus ochruros. In: Atlante degli Uccelli svernanti in provincia di Grosseto (inverni 1988/89-1993/94). Corsi F. \& Giovacchini P. (eds.). Amministrazione Provinciale di Grosseto, WWF Grosseto. Editrice Caletra: 176.

Corsi F. \& Anselmi G., 1991 - Analisi dei rapaci nel tratto di Maremma compreso tra la Bruna e Talamone (GR). Picus, 17: 127-133.

Corsi F. \& Anselmi G., 1994 - Ghiandaia marina Coracias garrulus: status, distribuzione, ecologia ed etologia nelle colonie della provincia di Grosseto. In: Atti del VI Convegno Italiano di Ornitologia, Torino 8-11 ottobre 1991. Baldaccini N.E., Mingozzi T. \& Violani C. (eds.). Museo regionale di Scienze Naturali: 503-504.

Corsi F. \& Colli M., 2013 - Le Gru, Grus grus, in inverno nella Maremma Grossetana. Gli Uccelli d'Italia, 38: 110-113.

Corsi F. \& Dragonetti M., 2010 - Svernamento delle Gru Grus grus nei dintorni di Grosseto. Picus, 36: 104-106

Corsi F. \& Giovacchini P. (eds.), 1995a - Atlante degli Uccelli svernanti in provincia di Grosseto (inverni 1988/89-1993/94). Amministrazione Provinciale di Grosseto, WWF Grosseto. Editrice Caletra. 
Corsi F. \& Giovacchini P., 1995b - Alcuni aspetti eto-ecologici della garzaia della Diaccia Botrona. In: Atti VII Convegno Italiano di Ornitologia, Urbino 23-26 settembre 1993. Pandolfi M. \& Foschi U.F. (eds.). Supplemento Ricerche di Biolologia della Selvaggina, XXII: 615-616.

Corsi F. \& Giovacchini P., 2001 - Stato attuale delle conoscenze sul Cuculo dal ciuffo Clamator glandarius nella Maremma grossetana. In: Atti del XI Convegno Italiano di Ornitologia, Castiglioncello (Livorno) 26-30 settembre 2001. Tellini Florenzano G., Barbagli F. \& Baccetti N. (eds.). Avocetta, 25: 194.

Corsi F. \& Marcucci L., 1995 - Colombaccio Columba palumbus. In: Atlante degli Uccelli svernanti in provincia di Grosseto (inverni 1988/89-1993/94). Corsi F. \& Giovacchini P. (redattori). Amministrazione Provinciale di Grosseto, WWF Grosseto. Editrice Caletra: 151.

Di Carlo E.A., 1977 - Morfologia, tassonomia e distribuzione in Italia della Monachella (Oenanthe hispanica). Gli Uccelli d'Italia, 2: 5568.

Di Carlo E.A., 1981 - Ricerche ornitologiche sul litorale tirrenico del Lazio e Toscana. In: Ricerche ecologiche, floristiche e faunistiche sulla fascia costiera mediotirrenica italiana. Problemi attuali di Scienza e Cultura. Accademia Nazionale dei Lincei, Quaderni, 254: 77-236

Di Carlo E.A. \& Heinze J., 1975 - Notizie ornitologiche dal Lazio e Toscana. Rivista Italiana di Ornitologia, 45: 323-334.

Dinetti M., 1997a - Pettirosso Erithacus rubecula. In: Atlante degli uccelli nidificanti e svernanti in Toscana (1982-1992). Tellini Florenzano G., Arcamone E., Baccetti N., Meschini E. \& Sposimo P. (eds.). Quaderni del Museo di Storia Naturale di Livorno. Monografie, 1: 230-231.

Dinetti M., 1997b - Ballerina bianca Motacilla alba. In: Atlante degli uccelli nidificanti e svernanti in Toscana (1982-1992). Tellini Florenzano G., Arcamone E., Baccetti N., Meschini E. \& Sposimo P. (eds.). Quaderni del Museo di Storia Naturale di Livorno. Monografie, 1: 219-220.

Dottori R., 2008 - Aspetti della biologia riproduttiva della ghiandaia marina nel Parco Regionale della Maremma. Tesi di Laurea Magistrale in Scienze Naturali, Università degli Studi di Siena.

Dragonetti M., Corsi F., Farsi F., Passalacqua L. \& Giovacchini P., 2014 Roosting behaviour of Stone curlew Burhinus oedicnemus wintering in central Italy. Wader Study Group Bulletin, 121: 1-6.

Dragonetti M. \& Giovacchini P., 2009 - Aspects of breeding biology of Cattle Egret Bubulcus ibis in a Grosseto province colony (Tuscany, central Italy). Avocetta, 33 (2): 199-204.

Fabbrizzi F., 1995a - Astore Accipiter gentilis. In: Atlante degli Uccelli svernanti in provincia di Grosseto (inverni 1988/89-1993/94). Corsi F. \& Giovacchini P. (eds.). Amministrazione Provinciale di Grosseto, WWF Grosseto. Editrice Caletra: 88.

Fabbrizzi F., 1995b - Albanella reale Circus cyaneus. In: Atlante degli Uccelli svernanti in provincia di Grosseto (inverni 1988/891993/94). Corsi F. \& Giovacchini P. (eds.). Amministrazione Provinciale di Grosseto, WWF Grosseto. Editrice Caletra: 8687.

Farina A., 1977 - Fauna dell'Etruria settentrionale. Litostampa Sant'Agnese, Arezzo.

Farina A. \& Martelli C., 1980 - Breeding bird census of an italian mediterranean habitat: the Parco Naturale della Maremma. In: Bird census work and nature conservation. Proceedings of the VI International Conference of Bird Census Work and IV meeting of the European Ornithological Atlas Committee, Universität Göttingen, Bundesrepublik Deutschland, 24.-28.09.1979. Oelke H. (ed). Dachverband Deutscher Avifaunisten: 129-135.

Fasola M., Albanese G., ASOER, Boano G., Boncompagni E., Bressan U., Brunelli M., Ciaccio A., Floris G., Grussu M., Guglielmi R., Guzzon C., Mezzavilla F., Paesani G., Sacchetti A., Sanna M., Scarton F., Scoccianti C., Utmar P., Vaschetti G. \& Velatta F., 2007 Le Garzaie in Italia: 2002. Avocetta, 31: 5-46.

Fasola M. \& Alieri R., 1992 - Nitticora Nycticorax nycticorax. In: Fauna d'Italia. XXIX. Aves. I. Brichetti P., De Franceschi P. \& Baccetti N. (eds.). Edizioni Calderini.

Fasola M. \& Brichetti P., 1984 - Proposte per una terminologia ornitologica. Avocetta, 8: 119-125.
Ferlini F., 2015 - Evoluzione dell'areale riproduttivo della Cutrettola "italiana" Motacilla flava cinereocapilla. Rivista Italiana di Ornitologia, 85: 3-18.

Fornasari L., Londi G., Buvoli L., Tellini Florenzano G., La Gioia G., Pedrini P., Brichetti P. \& De Carli E., 2010 - Distribuzione geografica e ambientale degli uccelli comuni nidificanti in Italia, 20002004 (dati del progetto MITO 2000). Avocetta, 34: 5-224.

Foschi F., 1976 - Le Cutrettole italiane (Motacilla L., 1758). Gli Uccelli d'Italia, 1: 9-15.

Franceschi A., 2012 - Distribuzione e consistenza del Gabbiano reale, Larus michahellis, lungo la costa toscana. In: Atti del XIV Convegno italiano di Ornitologia, Trieste 26-30 settembre 2007. Benussi E. \& Perco Fa. (a cura di). Rivista Italiana di Ornitologia, 82: 223-226.

Gariboldi A., Rizzi V. \& Casale F., 2000 - Aree importanti per l'avifauna in Italia. LIPU, Ministero per le Politiche Agricole e Forestali.

Gill F. \& Donsker D. (eds.), 2018 - IOC World Birds List (v 8.2). $<\mathrm{http} / / /$ www.worldbirdnames.org/>

Giovacchini P., 1990a - Svernamento del Mignattaio (Plegadis falcinellus) nel litorale grossetano. Avocetta, 14: 136.

Giovacchini P., 1990b - Nuovi dati sulla permanenza del Falco pescatore, Pandion haliaetus, nel Parco Naturale della Maremma. Rivista Italiana di Ornitologia, 60: 203.

Giovacchini P., 1991 - Contributo alla conoscenza dell'avifauna della Voltina (GR): Podicipedidae, Anatidae, Rallidae. Atti del Museo civico di Storia naturale di Grosseto, 14: 15-24.

Giovacchini P., 1994 - Lo svernamento del Cormorano Phalacrocorax carbo sinensis nel Parco Naturale della Maremma nell'inverno 1990. In: Atti del VI Convegno Italiano di Ornitologia, Torino 8-11 ottobre 1991. Baldaccini N.E., Mingozzi T. \& Violani C. (eds.). Museo regionale Scienze Naturali: 467.

Giovacchini P., 1995a - Oca selvatica Anser anser. In: Atlante degli Uccelli svernanti in provincia di Grosseto (inverni 1988/89-1993/94). Corsi F. \& Giovacchini P. (eds.). Amministrazione Provinciale di Grosseto, WWF Grosseto. Editrice Caletra: 50-51.

Giovacchini P., 1995b - Cigno reale Cygnus olor. In: Atlante degli Uccelli svernanti in provincia di Grosseto (inverni 1988/89-1993/94). Corsi F. \& Giovacchini P. (eds.). Amministrazione Provinciale di Grosseto, WWF Grosseto. Editrice Caletra: 47.

Giovacchini P., 1995c - Le specie rilevate in periodi invernali diversi da quello d'inchiesta. In: Atlante degli Uccelli svernanti in provincia di Grosseto (inverni 1988/89-1993/94). Corsi F. \& Giovacchini P. (eds.). Amministrazione Provinciale di Grosseto, WWF Grosseto. Editrice Caletra: 241-245.

Giovacchini P., 1995d - Smergo maggiore Mergus merganser. In: Atlante degli Uccelli svernanti in provincia di Grosseto (inverni 1988/89-1993/94). Corsi F. \& Giovacchini P. (eds.). Amministrazione Provinciale di Grosseto, WWF Grosseto. Editrice Caletra: 80.

Giovacchini P., 1995e - Strolaga mezzana Gavia arctica. In: Atlante degli Uccelli svernanti in provincia di Grosseto (inverni 1988/891993/94). Corsi F. \& Giovacchini P. (eds.). Amministrazione Provinciale di Grosseto, WWF Grosseto. Editrice Caletra: 26-27.

Giovacchini P., 1995f - Falco pescatore Pandion haliaetus. In: Atlante degli Uccelli svernanti in provincia di Grosseto (inverni 1988/891993/94). Corsi F. \& Giovacchini P. (eds.). Amministrazione Provinciale di Grosseto, WWF Grosseto. Editrice Caletra: 94.

Giovacchini P., 1995g - Gru Grus grus. In: Atlante degli Uccelli svernanti in provincia di Grosseto (inverni 1988/89-1993/94). Corsi F. \& Giovacchini P. (eds.). Amministrazione Provinciale di Grosseto, WWF Grosseto. Editrice Caletra: 109.

Giovacchini P., 1995h - Gabbiano comune Larus ridibundus. In: Atlante degli Uccelli svernanti in provincia di Grosseto (inverni 1988/89-1993/94). Corsi F. \& Giovacchini P. (eds.). Amministrazione Provinciale di Grosseto, WWF Grosseto. Editrice Caletra: 137-138.

Giovacchini P., 1995i - Sterna comune Sterna hirundo. In: Atlante degli Uccelli svernanti in provincia di Grosseto (inverni 1988/891993/94). Corsi F. \& Giovacchini P. (eds.). Amministrazione Provinciale di Grosseto, WWF Grosseto. Editrice Caletra: 147.

Giovacchini P., 19951 - Corvo comune Corvus frugilegus. In: Atlante degli Uccelli svernanti in provincia di Grosseto (inverni 1988/891993/94). Corsi F. \& Giovacchini P. (eds.). Amministrazione Provinciale di Grosseto, WWF Grosseto. Editrice Caletra: 208. 
Giovacchini P., 1995m - Sordone Prunella collaris. In: Atlante degli Uccelli svernanti in provincia di Grosseto (inverni 1988/891993/94). Corsi F. \& Giovacchini P. (eds.). Amministrazione Provinciale di Grosseto, WWF Grosseto. Editrice Caletra: 173.

Giovacchini P., 1999 - Nuove specie per l'avifauna del Parco Regionale della Maremma (Grosseto). In: Atti del X Convegno italiano di Ornitologia, Caorle (VE) 23-26 settembre 1999. Scarton F., Fracasso G. \& Bogliani G. (eds.). Avocetta, 23: 181.

Giovacchini P., 2005 - La Cicogna nera in Toscana. In: La Cicogna nera Ciconia nigra in Italia. Bordignon L. (red.). Ente Parco Regionale Monte Fenera e Regione Piemonte.

Giovacchini P., 2006 - Uccelli. In: Check-list della fauna del Parco Regionale della Maremma. Plantamura G. \& Manganelli G. (a cura di), Lovari S. (coord.). Istituto di Biologia della Conservazione. Polo Universitario Grossetano, Ente Parco Regionale della Maremma.

Giovacchini P., 2014 - Sull'avifauna rupicola in alcune cave di parete dismesse della Toscana meridionale. Annali del Museo Civico di Storia Naturale di Genova "G. Doria”, 106: 75-91.

Giovacchini P. \& Anselmi G., 1990 - Osservazioni di Fistione turco (Netta rufina Pallas, 1773) nel Parco Naturale della Maremma. Atti del Museo civico di Storia naturale di Grosseto, 13: 35-36.

Giovacchini P. \& Celletti S., 1995 - Gabbiano reale Larus cachinnans. In: Atlante degli Uccelli svernanti in provincia di Grosseto (inverni 1988/89-1993/94). Corsi F. \& Giovacchini P. (eds.). Amministrazione Provinciale di Grosseto, WWF Grosseto. Editrice Caletra: 143.

Giovacchini P. \& Celletti S., 1997 - Il Falco della regina (Falco eleonorae) in provincia di Grosseto: risultati di una indagine storica ed attuale (Aves, Falconidae). Atti del Museo di Storia Naturale della Maremma, 16: 75-79.

Giovacchini P. \& Corsi F., 1993 - Sulla presenza del Fenicottero (Phoenicopterus ruber) nella Palude della Trappola (GR) nell'inverno 1966-67. Atti del Museo civico di Storia naturale di Grosseto, 15: 163-164.

Giovacchini P., Corsi F. \& Manfreda V., 2004 - Analisi e rassegna aggiornata delle osservazioni di Cuculo dal ciuffo Clamator glandarius nella Maremma grossetana. Alula, 11: 98-104.

Giovacchini P., Dragonetti M., Corsi F. \& Farsi F., 2012 - Monitoraggio invernale dell'Occhione (Burhinus oedicnemus) in provincia di Grosseto con l'uso del playback. In: Atti del XIV Convegno italiano di Ornitologia, Trieste 26-30 settembre 2007. Benussi E. \& Perco F. (eds.). Rivista Italiana di Ornitologia, 82: 80-84.

Giovacchini P., Dragonetti M., Fastelli P., Passalacqua L., Porciani M. \& Radi G., 2017 - Stato delle garzaie in provincia di Grosseto sino all'anno 2016. Abstract XIX Convegno italiano di Ornitologia, Torino 27 settembre-1 ottobre 2017. Fasano S. G. \& Rubolini D. (eds.). Tichodroma, 6: 151

Giovacchini P., Manfreda V. \& Moroni C., 2001 - Primi casi di nidificazione per Podiceps cristatus (L.) e Bubulcus ibis (L.) in provincia di Grosseto. Atti del Museo di Storia Naturale della Maremma, 19: 89-92.

Giovacchini P., Melini D. \& Stefanini P., 2011 - Il Fratino Charadrius alexandrinus nidificante in provincia di Grosseto (2008-2009): stato attuale della popolazione e analisi della distribuzione. In: Il Fratino status, biologia e conservazione di una specie minacciata. Atti del Convegno Nazionale. Bracciano (Roma), 18 settembre 2010. Biondi M. \& Pietrelli L. (a cura di). Edizioni Belvedere: 95-103.

Giovacchini P. \& Stefanini P., 2008 - La Protezione della Natura in Toscana. Siti di Importanza Regionale e Fauna Vertebrata nella provincia di Grosseto. Provincia di Grosseto, UOC Aree Protette e Biodiversità. Quaderni delle Aree Protette n 3. Tipografia Agnesotti.

Giovacchini P. \& Stefanini P., 2015 - Dieci anni di censimenti (20062015) dell'avifauna nidificante ed estivante nella ZPS "Padule di Diaccia Botrona". Abstract XVIII Convegno italiano di Ornitologia, Caramanico Terme (PE), 17-20 settembre 2015. De Sanctis A. \& Rubolini D. (eds.): 54. <https://cio2015.files.wordpress. com/2015/09/libroabstractconvegnoitalianoornitologiaaltarisoluzione.pdf>
Giusti F., Favilli L. \& Manganelli G., 1993 - La Fauna. In: La storia naturale della Toscana meridionale. Giusti F. (eds.). Monte dei Paschi di Siena, Amilcare Pizzi Editore: 343-439.

Guerrini G., 1981 - Il Parco della Maremma. Libreria Editrice Tellini.

La Gioia G., Melega L. \& Fornasari L., 2017 - Piano d'Azione Nazionale per il Grillaio (Falco naumanni). Quaderni di Conservazione della Natura. 41. Ministero dell'Ambiente e della Tutela del Territorio e del Mare. Istituto Superiore per la Protezione e la Ricerca Ambientale.

Lambertini M., 1997 - Gabbiano corso Larus audouinii. In: Atlante degli uccelli nidificanti e svernanti in Toscana (1982-1992). Tellini Florenzano G., Arcamone E., Baccetti N., Meschini E. \& Sposimo P. (eds.). Quaderni del Museo di Storia Naturale di Livorno. Monografie, 1: 149-151.

Liberatori F., Penteriani V. \& Pinchera F., 1988 - Censimento della popolazione di Falco di palude, Circus aeruginosus, nella Palude della Diaccia Botrona (Toscana). Rivista Italiana di Ornitologia, 58: 49-52.

Lovari S. \& Martelli C., 1977 - Osservazioni sull'avifauna del Parco Naturale della Maremma, con particolare riferimento ai Falconiformes. In: Atti del convegno: Contributi alla conoscenza del paesaggio e dei problemi del Parco Naturale della Maremma. Arrigoni P. V., Gellini R., Innamorati M., Lenzi Grillini C., Piussi P., Sartori G., Lovari S., Renzoni A. \& Sanesi G. (eds.). Informatore Botanico Italiano, 9: 156-163.

Mainardi R., 1993-1994 - La riproduzione del Fratino Charadrius alexandrinus lungo la costa toscana. Quaderni del Museo di Storia Naturale di Livorno, 13: 31-51.

Mainardi R., 1997a - Pavoncella Vanellus vanellus. In: Atlante degli uccelli nidificanti e svernanti in Toscana (1982-1992). Tellini Florenzano G., Arcamone E., Baccetti N., Meschini E. \& Sposimo P. (eds.). Quaderni del Museo di Storia Naturale di Livorno. Monografie, 1: 129-130.

Mainardi R., 1997b - Piviere dorato Pluvialis apricaria. In: Atlante degli uccelli nidificanti e svernanti in Toscana (1982-1992). Tellini Florenzano G., Arcamone E., Baccetti N., Meschini E. \& Sposimo P. (eds.). Quaderni del Museo di Storia Naturale di Livorno. Monografie, 1: 127.

Martelli C., 1980 - Alimentazione del Barbagianni Tyto alba (Scopoli) nel Parco Naturale della Maremma. Avocetta, 4: 75-81.

Martelli C., 1983 - I Rapaci. Guida alla conoscenza dell'ambiente del Parco Naturale della Maremma. Volume 2. Editori Del Grifo.

Martucci O. \& Giovacchini P., 1994 - Some aspects of the feeding habits of the Cormorant (Phalacrocorax carbo sinensis) wintering in the Maremma Natural Park (Grosseto-Central Italy). Avocetta, 18: 53-56.

Mellone U. \& Sighele M. (eds.), 2006 - Resoconto Ornitologico Italiano. Anno 2005. Avocetta, 31: 81-86

Mellone U. \& Sighele M. (eds.), 2007 - Resoconto Ornitologico Italiano. Anno 2006. Avocetta, 31: 79-86.

Mellone U., Sighele M. \& Arcamone E. (eds.), 2005 - Resoconto Ornitologico Italiano. Anno 2004. Avocetta, 29: 98-102.

Melotti P. \& Spagnesi M., 1979 - Analisi delle riprese di Falco pescatore (Pandion h. haliaetus L.) avvenute in Italia nel periodo 19391977. Ricerche di Biologia della Selvaggina, 65: 1-19.

Meschini A. \& Fraschetti F., 1989 - Distribuzione, consistenza e habitat dell'Occhione Burhinus oedicnemus in Lazio e Toscana. Avocetta, 13: $15-20$.

Mingozzi T., Storino P., Venuto G., Alessandria G., Arcamone E., Urso S., Ruggieri L., Massetti L. \& Massolo A., 2013 - Autumn migration of Common Cranes Grus grus through the Italian Peninsula: new vs. historical flyways and their meteorological correlates. Acta Ornithologica, 48: 165-177.

Moltoni E., 1966 - Altre notizie su uccelli inanellati all'estero e ripresi in Italia ed in Libia. Rivista Italiana di Ornitologia, 36: 109-310.

Moltoni E., 1973 - Elenco di parecchie centinaia di uccelli inanellati all'estero e ripresi in Italia ed in Libia. Rivista Italiana di Ornitologia, 43 (suppl): 1-182.

Moltoni E., 1975 - L'Avifauna dell'Isola di Capraia (Arcipelago toscano). Rivista Italiana di Ornitologia, 45: 97-217.

Moltoni E., 1976 - Nuovi dati su uccelli inanellati all'estero e ripresi in Italia ed in Libia. Rivista Italiana di Ornitologia, 46 (suppl): 1-71. 
Moltoni E. \& Brichetti P., 1978 - Elenco degli Uccelli Italiani. Rivista Italiana di Ornitologia, 48: 65-142.

Monti F., 2012a - Composizione e ricchezza specifica dell'avifauna acquatica della foce del fiume Ombrone (Toscana, GR). Atti del Museo di Storia Naturale della Maremma, 23: 49-55.

Monti F., 2012b - State of knowledge and population trends of the Osprey Pandion haliaetus in the Mediterranean basin. In: Proceeding of the 13th Medmaravis Pan-Mediterranean Symposium. Yésou P., Baccetti N. \& Sultana J. (eds.). Medmaravis: 195-201.

Monti F., Dominici J.M., Choquet R., Duriez O., Sammuri G. \& Sforzi A., 2014 - The Osprey reintroduction in Central Italy: dispersal, survival and first breeding data. Bird Study, 61: 465-473.

Monti F., Grémillet D., Sforzi A., Sammuri G., Dominici J.M., Triay R., Munoz A., Fusani L. \& Duriez O., 2018 - Migration and wintering strategies in vulnerable Mediterranean Osprey populations. Ibis, 160: 554-567.

Morimando F., Nardi R. \& Sammuri G., 2008 - Guida ai rapaci diurni del Parco Regionale della Maremma. Reda Edizioni

Nardelli R., Andreotti A., Bianchi E., Brambilla M., Brecciaroli B., Celada C., Dupré E., Gustin M., Longoni V., Pirrello S., Spina F., Volponi S. \& Serra L., 2015 - Rapporto sull'applicazione della Direttiva 147/2009/CE in Italia: dimensione, distribuzione e trend delle popolazioni di uccelli (2008-2012). ISPRA, Serie Rapporti, 219.

Occhiato D., 2003 - Pine Bunting in Italy: status and distribution. Dutch Birding, 25: 32-39.

Paoloni S., Dragonetti M. \& Giovacchini P., 2018 - Ecological preferences, behavior observations of Cattle Egret Bubulcus ibis and their interactions with the cattle in the Maremma Regional Park (Tuscany, central Italy). Avocetta, 42: 15-20.

Passalacqua L., Anselmi G., Bevilacqua L., Cappelli A., Corsi F., Dragonetti M., Falchi V., Farsi F., Giovacchini P., Lamberti M., Paoloni S., Porciani M., Radi G., Santi A., Vignali S. \& Fastelli P., 2015 - I dormitori invernali di Ardeinae nella Maremma grossetana: distribuzione e analisi delle presenze in una indagine a medio-lungo periodo. Abstract XVIII Convegno Italiano di Ornitologia, Caramanico Terme (PE) 17-20 settembre 2015. De Sanctis A. \& Rubolini D. (eds.): 65-66. <https://cio2015.files. wordpress.com/2015/09/libroabstractconvegnoitalianoornitologiaaltarisoluzione.pdf $>$

Patterson I.J., Cavallini P. \& Rolando A., 1991 - Density, range size and diet of the European Jay Garrulus glandarius in the Maremma Natural Park, Tuscany, Italy, in summer and autumn. Ornis Scandinavica, 22: 79-87

Perco F., 1992 - Oca selvatica Anser anser. In: Fauna d'Italia. XXIX. Aves. I. Brichetti P., De Franceschi P. \& Baccetti N. (eds.). Edizioni Calderini.

Peronace V., Cecere J.G., Gustin M. \& Rondinini C., 2012 - Lista Rossa 2011 degli Uccelli Nidificanti in Italia. Avocetta, 36: 11-58.

Petretti F., 2008 - L'Aquila dei serpenti. Pandion Edizioni.

Petretti F., 2013 - Nidificazione di Aquila minore Aquila pennata in Toscana. Alula, 20: 144-146.

Petretti F. \& Rizzo Pinna V., 2010 - Il Biancone (Circaetus gallicus) nel Parco Regionale della Maremma. In: Biodiversità Specifiche e Funzionali degli Ecosistemi Rilevanti [BioSFER]. Sforzi A. (ed). Report finale. Ente Parco Regionale della Maremma.

Pezzo F., 1997a - Airone bianco maggiore Egretta alba. In: Atlante degli uccelli nidificanti e svernanti in Toscana (1982-1992). Tellini Florenzano G., Arcamone E., Baccetti N., Meschini E. \& Sposimo P. (eds.). Quaderni del Museo di Storia Naturale di Livorno. Monografie, 1: 47.

Pezzo F., 1997b - Gufo comune Asio otus. In: Atlante degli uccelli nidificanti e svernanti in Toscana (1982-1992). Tellini Florenzano G., Arcamone E., Baccetti N., Meschini E. \& Sposimo P. (eds.). Quaderni del Museo di Storia Naturale di Livorno. Monografie, 1: $178-179$.

Pezzo F., 2012 - La comunità ornitica nidificante nella "Pineta Granducale di Alberese" (Toscana). Composizione, struttura e indicazioni gestionali per la conservazione. Atti del Museo di Storia Naturale della Maremma, 23: 91-101.

Pezzo F., Chiancianesi G., Cutini S., Fabbrizzi F., Grilli G., Nardi R. \& Paesani G., 2016 - Rapporto sullo status del Lanario Falco biarmicus feldeggii in Toscana 2014-2016. Picus, 42: 132-136.
Pezzo F. \& Cianchi F., 2015 - La Ghiandaia marina Coracias garrulus nella Maremma Toscana: esperienze di conservazione a confronto. In: Atti del I Convegno nazionale sulla Ghiandaia marina Coracias garrulus "Un lampo turchese di interesse comunitario", 20 settembre 2014, Canale Monterano (RM). Meschini A. \& CORACIAS (a cura di). Alula, 22: 79-85.

Picciau A., 2017 - Differenti strategie di migrazione di popolazioni di occhione (Burhinus oedicnemus) dell'Italia centrale e settentrionale. Tesi di Laurea Magistrale in Scienze Naturali, Università degli Studi di Bologna.

Porciani M., 1995 - Frosone Coccothraustes coccothraustes. In: Atlante degli Uccelli svernanti in provincia di Grosseto (inverni 1988/891993/94). Corsi F. \& Giovacchini P. (eds.). Amministrazione Provinciale di Grosseto, WWF Grosseto. Editrice Caletra: 226.

Puglisi L. \& Meschini E., 2015 - Andamento della popolazione nidificante di Fratino Charadrius alexandrinus in Toscana: indicazioni per la sua conservazione. Picus, 41: 83-95.

Puglisi L. \& Meschini E., 2016 - Situazione critica per il Fratino Charadrius alexandrinus nidificante in Toscana. Picus, 42: 137-141.

Puglisi L., Pezzo F. \& Sacchetti A., 2012 - Gli aironi coloniali in Toscana: andamento, distribuzione e conservazione. Edizioni Regione Toscana, Centro Stampa Giunta Regionale.

Rete Rurale Nazionale \& LIPU, 2015 - Uccelli comuni in Italia. Aggiornamento degli andamenti di popolazione e del Farmland Bird Index per la Rete Rurale Nazionale dal 2000 al 2014. Ministero delle Politiche Agricole e Forestali.

Ridolfi C., 1908 - Collezione ornitologica di Meleto in Val d'Elsa. Tipografia M. Ricci.

Rigacci L., 1993 - Il Gufo reale in Toscana. Serie scientifica n. 1. WWF Delegazione Toscana.

Rolando A., 1998 - Factors affecting movements and home ranges in the jay (Garrulus glandarius). Journal of the Zoological Society of London, 246: 249-257.

Rolando A., Alemanni D., Brezzo L. \& Palestrini C., 1997 - Uso dell'habitat e aree familiari della Ghiandaia Garrulus glandarius nel Parco Naturale della Maremma in periodo invernale. In: Atti del IX Convegno Italiano di Ornitologia, Alghero (SS) 9-12 ottobre 1997. Bogliani G., Galeotti P. \& Torre A. (eds.). Avocetta, 21: 137.

Rolando A., Cavallini P., Cursano B. \& Olsen A., 1995 - Non-territorial behaviour and habitat selection in the Jay Garrulus glandarius in a Mediterranean coastal area during the reproductive period. Journal of Avian Biology, 26: 154-161.

Romè A., 1978 - Il Cavaliere d'Italia Himantopus himantopus (L.) in Toscana. Atti Società Toscana Scienze Naturali, Serie B, 85: 131137.

Romè A., 1980a - Il Fenicottero (Phoenicopterus ruber roseus Pallas) in Toscana. Rivista Italiana di Ornitologia, 50: 158-166.

Romè A., 1980b - La Cutrettola (Motacilla flava L.) lungo il litorale tirrenico-toscano. Natura, 71: 21-27.

Romè A., 1981 - La Garzetta Egretta g. garzetta (L.) in Toscana. Atti Società Toscana Scienze Naturali, Serie B, 88: 163-173.

Romè A., 1982 - Il Tarabuso (Botaurus stellaris, L.) in Toscana. In: Atti I Convegno Italiano di Ornitologia, Aulla (MS) 10-15 settembre 1981. Farina A. (a cura di). Regione Toscana, Centro Italiano Studi Ornitologici, Museo di Storia Naturale della Lunigiana: $178-180$.

Romè A., 1983a - Nidificazione dell'Airone cenerino (Ardea cinerea) nella Palude di Castiglione della Pescaia (Grosseto) e sua distribuzione in Toscana. Rivista Italiana di Ornitologia, 53: 153-160.

Romè A., 1983b - Indagine sulla Pernice di mare Glareola pratincola in Toscana. Gli Uccelli d'Italia, 8: 36-41.

Romè A. \& Travison G., 1982a - Nuovi dati sul Cavaliere d'Italia ( $\mathrm{Hi}$ mantopus himantopus) in Italia. Rivista Italiana di Ornitologia, 52: 85-90.

Romè A. \& Travison G., 1982b - Nidificazione della Rondine rossiccia Hirundo daurica nel Parco Naturale della Maremma e sua distribuzione in Toscana. Gli Uccelli d'Italia, 7: 48-52.

Santolini R. \& Pandolfi M., 1992 - Smergo maggiore Mergus merganser. In: Fauna d'Italia. XXIX. Aves. I. Brichetti P., De Franceschi P. \& Baccetti N (eds.). Edizioni Calderini. 
Savio R., 1997 - Poiana Buteo buteo. In: Atlante degli uccelli nidificanti e svernanti in Toscana (1982-1992). Tellini Florenzano G., Arcamone E., Baccetti N., Meschini E. \& Sposimo P. (eds.). Quaderni del Museo di Storia Naturale di Livorno. Monografie, 1: 93-94.

Scapini F. \& Nardi M. (a cura di), 2007 - Il Parco Regionale della Maremma e il suo territorio. Pacini Editore.

Scoccati M., 2014 - Sito di nidificazione e conservazione della ghiandaia marina nel Parco Regionale della Maremma. Tesi di Laurea Magistrale in Scienze Naturali, Università degli Studi di Siena.

Scoccianti C. \& Scoccianti G., 1995 - I rapaci diurni delle Province di Siena e Grosseto. Studio per la conservazione. WWF Toscana. Serie Scientifica n. 2.

Serra L., 1997 - Corriere grosso Charadrius hiaticula. In: Atlante degli uccelli nidificanti e svernanti in Toscana (1982-1992). Tellini Florenzano G., Arcamone E., Baccetti N., Meschini E. \& Sposimo P. (eds.). Quaderni del Museo di Storia Naturale di Livorno. Monografie, 1: 124.

Serra L. \& Baccetti N., 1991 - La migrazione primaverile del Combattente (Philomachus pugnax) e della Pittima reale (Limosa limosa) in Italia settentrionale: descrizione delle ricerche in corso e proposte per la conservazione delle aree di sosta. In: Atti del II Convegno Nazionale del Biologi della Selvaggina, Bologna 7-9 marzo 1991. Spagnesi M. \& Toso S. (a cura di). Supplemento Ricerche di Biologia della Selvaggina, XIX: 181-194.

Sforzi A., 2013 - Piano di Gestione dei SIC/SIR IT51A0039 [SIR 113 e A113(ZPS)] Palude della Trappola e Bocca d'Ombrone; IT51 A0014 [SIR 114] Pineta Granducale dell'Uccellina; IT51A0015 [SIR 115] Dune costiere del Parco dell'Uccellina. Ente Parco Regionale della Maremma.

Sighele M. \& Janni O. (a cura di), 2009 - Resoconto Ornitologico Italiano. Anno 2007. Avocetta, 33: 123-136.

Smit C. J., 1986 - Waders along the Mediterranean. A summary of present knowledge. In: First conference on Birds Wintering in the Mediterranean Region. Proceeding of an International Conference held at Aulla Italy, February 23-25, 1984. Farina A. (ed). Istituto Nazionale di Biologia della selvaggina, Supplemento alle Ricerche di Biologia della Selvaggina, X: 297-317.

Società Botanica Italiana (ed.), 1971 - Censimento dei biotopi di rilevante interesse vegetazionale meritevoli di conservazione in Italia. Tipografia succ. Savini-Mercuri, Camerino (MC).

Spanò S. \& Baccetti N., 1992 - Sula Sula bassana. In: Fauna d'Italia. XXIX. Aves. I. Brichetti P., De Franceschi P. \& Baccetti N (eds.). Edizioni Calderini.

Spina F., Massi A., Montemaggiori A. \& Baccetti N., 1993 - Spring migration across central mediterranean: general results from the "Progetto Piccole Isole". Die Vogelwarte, 37: 1-94.

Sposimo P., 1997a - Sterpazzola Sylvia communis. In: Atlante degli uccelli nidificanti e svernanti in Toscana (1982-1992). Tellini Florenzano G., Arcamone E., Baccetti N., Meschini E. \& Sposimo P. (eds.). Quaderni del Museo di Storia Naturale di Livorno. Monografie, 1: 273.

Sposimo P., 1997b - Tordo sassello Turdus iliacus. In: Atlante degli uccelli nidificanti e svernanti in Toscana (1982-1992). Tellini Florenzano G., Arcamone E., Baccetti N., Meschini E. \& Sposimo P. (eds.). Quaderni del Museo di Storia Naturale di Livorno. Monografie, 1: 250.

Sposimo P., 1997c - Tordo bottaccio Turdus philomelos. In: Atlante degli uccelli nidificanti e svernanti in Toscana (1982-1992). Tellini Florenzano G., Arcamone E., Baccetti N., Meschini E. \& Sposimo P. (eds.). Quaderni del Museo di Storia Naturale di Livorno. Monografie, 1: 248-249.

Sposimo P., 1997d - Saltimpalo Saxicola torquata. In: Atlante degli uccelli nidificanti e svernanti in Toscana (1982-1992). Tellini Florenzano G., Arcamone E., Baccetti N., Meschini E. \& Sposimo P. (eds.). Quaderni del Museo di Storia Naturale di Livorno. Monografie, 1: 237-238.

Staneva A. \& Burfield I., 2017 - European birds of conservation concern: populations, trends and national responsibilities. BirdLife International.

Svensson L., 2013 - A taxonomic revision of the Subalpine Warbler Sylvia cantillans. Bulletin of the British Ornithologists' Club, 113: 240-248.
Tellini Florenzano G., 1997a - Picchio rosso maggiore Picoides major. In: Tellini Florenzano G., Arcamone E., Baccetti N., Meschini E. \& Sposimo P. (eds.). Atlante degli Uccelli nidificanti e svernanti in Toscana (1982-1992). Quaderni del Museo di Storia Naturale di Livorno. Monografie, 1: 194-195.

Tellini Florenzano G., 1997b - Fiorrancino Regulus ignicapillus. In: Tellini Florenzano G., Arcamone E., Baccetti N., Meschini E. \& Sposimo P. (eds.). Atlante degli Uccelli nidificanti e svernanti in Toscana (1982-1992). Quaderni del Museo di Storia Naturale di Livorno. Monografie, 1: 283-284.

Tellini Florenzano G., Arcamone E., Baccetti N., Meschini E. \& Sposimo P. (eds.), 1997 - Atlante degli Uccelli nidificanti e svernanti in Toscana (1982-1992). Quaderni del Museo di Storia Naturale di Livorno. Monografie, 1.

Tinarelli R., Alessandria G., Giovacchini P., Gola L., Ientile R., Meschini A., Nissardi S., Parodi R., Perco F., Taiariol P.G. \& Zucca C., 2009 - Consistenza e distribuzione dell'occhione in Italia: aggiornamento al 2008. In: Atti della giornata di studio "L'Occhione (Burhinus oedicnemus): biologia e conservazione di una specie di interesse comunitario. Indicazioni per la gestione del territorio e delle aree protette". Corte di Giarola, 20 settembre 2008. Giunchi D., Pollonara E. \& Baldaccini N. E. (eds.). Parco Fluviale del Taro, Quaderni di documentazione, 7: 45-50.

Toso S. (ed.), 1983 - Nuovi avvistamenti. Avocetta, 7: 59-66.

Trotta M., 2011 - Analisi dei fattori di disturbo nelle aree di alimentazione del chiurlo maggiore Numenius arquata in un sito di svernamento dell'Italia centrale. Avocetta, 35: 19-22.

Valle R., Piacentini D., Scarton F., Serra L., Grussu M., Corso A. \& Utmar P., 1995 - Status e distribuzione della Volpoca Tadorna tadorna nidificante in Italia. In: Atti VIII Convegno italiano di Ornitologia, Pavia 7-10 settembre 1995. Fasola M. \& Saino N. (eds.). Avocetta, 19: 163.

Valtriani M., 1994 - "Via dalla pazza folla": caratterizzazione di una piccola popolazione svernante di Piovanello pancianera (Calidris alpina) insediata lungo il litorale toscano. Tesi di Laurea Magistrale in Scienze Biologiche, Università di Pisa.

Zenatello M., Baccetti N. \& Borghesi F., 2014 - Risultati dei censimenti degli uccelli acquatici svernanti in Italia. Distribuzione, stima e trend delle popolazioni nel 2001-2010. ISPRA, Serie Rapporti, 206. 